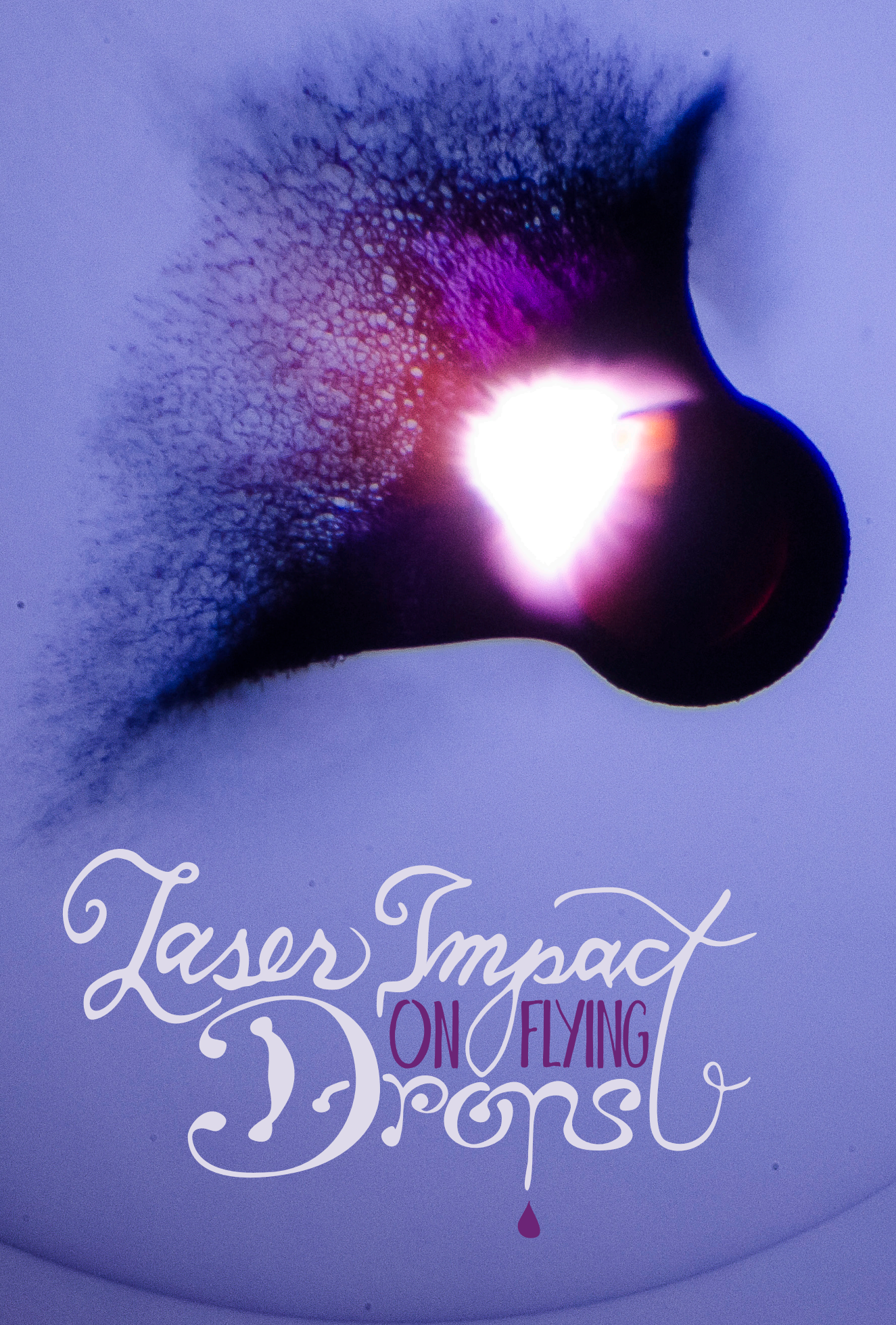

ALeXANDER LUdWIG KLEIN 



\section{LASER IMPACT ON}

\section{FLYING DROPS}

Alexander Ludwig Klein 


\section{Graduation committee}

Prof. dr. ir. J.W.M. Hilgenkamp (Chairman) University of Twente Prof. dr. rer. nat. D. Lohse (Supervisor)

Dr. ir. H. Gelderblom (CO-Supervisor) University of Twente Prof. dr. ir. J.P.H. Benschop

Prof. dr. A.M. Versluis Prof. dr.-ing. W. Schröder Ass. prof. dr. N. Bremond

University of Twente University of Twente \& ASML University of Twente RWTH Aachen University ESPCI Paris

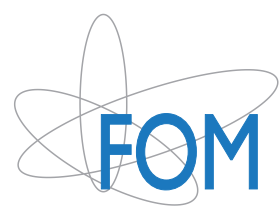

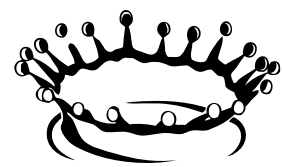

Physics of Fluids

\section{ASML}

The work in this thesis was carried out at the Physics of Fluids group of the Faculty of Science and Technology of the University of Twente. It is part of the Industrial Partnership Programme (IPP) "FEUL" of the Foundation for Fundamental Research on Matter (FOM, from January 2017 NWO), together with ASML.

Dutch title: Laser inslag op vliegende druppels

Cover: An image of the laser impact on a flying drop as explained in $\S 1$ (front) and the drop fragmentation from $\S 7$ (back) - artwork by Silvia Baumeier

Publisher:

Alexander L. Klein, Physics of Fluids, University of Twente, P.O. Box 217, 7500 AE Enschede, The Netherlands pof.tnw.utwente.nl

Printed by: Gildeprint - The Netherlands

(c) Alexander L. Klein, Enschede, The Netherlands, 2017

No part of this work may be reproduced by print, photocopy, or any other means without the permission in writing from the publisher ISBN: 978-90-365-4342-2 \& DOI: 10.3990/1.9789036543422 


\title{
LASER IMPACT ON FLYING DROPS
}

\author{
DISSERTATION
}

to obtain

the degree of doctor at the University of Twente, on the authority of the rector magnificus,

Prof. dr. T.T.M. Palstra,

on account of the decision of the graduation committee

to be publicly defended

on Friday $23^{\text {th }}$ of June 2017 at $16: 45$

$$
\text { by }
$$

Alexander Ludwig Klein

Born on the $1^{\text {st }}$ of December 1981

in Bad Honnef, Germany 
This dissertation has been approved by the supervisor:

Prof. dr. rer. nat. D. Lohse

and the co-supervisor Dr. ir. H. Gelderblom 


\section{Contents}

1 Introduction 1

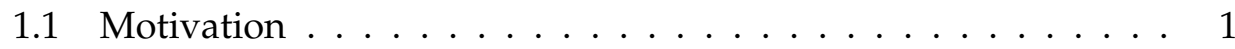

1.2 EUV Nanolithography . . . . . . . . . . . . . . 3

1.3 Fluid dynamics in EUV light sources . . . . . . . . . . . . . 6

1.4 A guide through the thesis . . . . . . . . . . . . . . 10

2 Laser impact on a drop $\quad 11$

3 Experimental setup $\quad 15$

3.1 Introduction . . . . . . . . . . . . . . . 16

3.2 System overview . . . . . . . . . . . . . . . . . . . . . . . . . . . . . . 18

3.3 Laser system and free beam propagation . . . . . . . . . . . 19

3.4 Beam shaping and near-field imaging . . . . . . . . . . 23

3.5 Visualization . . . . . . . . . . . . . . . . . 28

3.6 Drop impact chamber . . . . . . . . . . . . . . . . . . . 31

3.7 Liquids . . . . . . . . . . . . . . . . . 33

3.8 System control and timing . . . . . . . . . . . . . 37

3.9 Results . . . . . . . . . . . . . . . . . . 42

3.10 Summary . . . . . . . . . . . . . . . 51

4 Vapor propulsion of absorbing millimeter-sized drops 53

4.1 Introduction . . . . . . . . . . . . . . . 54

4.2 Experimental methods . . . . . . . . . . . . . . . 54

4.3 Results \& interpretation . . . . . . . . . . . . . . . 57

4.4 Numerical results . . . . . . . . . . . . . . . . . . . . 62

4.5 Conclusions . . . . . . . . . . . . . . . . 64

4.A Measurement of energy absorption . . . . . . . . . . . . . 65

4.B Measurement of drop displacement . . . . . . . . . 66

5 Plasma propulsion of metallic micrometer-sized drops $\quad 67$

5.1 Introduction . . . . . . . . . . . . . . . 67

5.2 Experimental methods . . . . . . . . . . . . . 68 


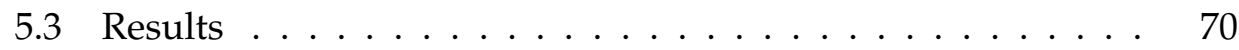

5.4 Conclusions . . . . . . . . . . . . . . . . . 80

6 Drop deformation by laser-pulse impact $\quad 81$

6.1 Introduction . . . . . . . . . . . . . . . 82

6.2 Problem formulation \& methods . . . . . . . . . . . . . 85

6.3 Results . . . . . . . . . . . . . . . . . . 88

6.4 Late time dynamics: the thin sheet limit . . . . . . . . . . . 99

6.5 Conclusion . . . . . . . . . . . . . . . . . . . . . 104

6.A Uniform laser beam profile results in a flat drop . . . . . . . 105

6.B Kinetic-energy partition in experiments . . . . . . . . . 106

$\begin{array}{lll}7 & \text { Drop fragmentation by laser-pulse impact } & 109\end{array}$

7.1 Introduction . . . . . . . . . . . . . . . . . . 110

7.2 Liquid systems . . . . . . . . . . . . . . . . 112

7.3 Experimental setups . . . . . . . . . . . . . . . . 115

7.4 Chronology . . . . . . . . . . . . . . . . . . . 121

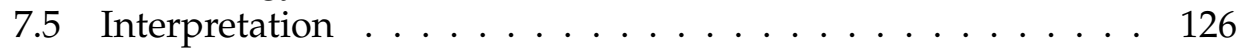

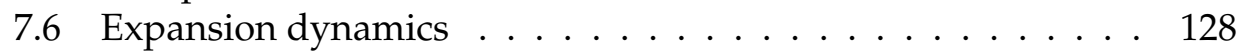

7.7 Rim breakup . . . . . . . . . . . . . . . . . 133

7.8 Sheet breakup . . . . . . . . . . . . . . . 138

7.9 Discussion \& conclusion . . . . . . . . . . . . . . . . . . . . 151

7.A Rescaling the late-time sheet model . . . . . . . . . . . 157

8 Summary and Outlook 159

$\begin{array}{ll}\text { References } & 165\end{array}$

$\begin{array}{ll}\text { Samenvatting } & 175\end{array}$

$\begin{array}{ll}\text { Acknowledgements } & 179\end{array}$

$\begin{array}{ll}\text { About the author } & 183\end{array}$ 


\section{1 \\ Introduction}

\subsection{Motivation}

Our society produced major scientific and technological breakthroughs in the last three decades such as in renewable energy resources [1], in biomedicine with the Human Genome Project [2], and in advanced materials by exploring man-made photonic metamaterials [3]. International efforts to answer some of the fundamental open questions are large-scale experimental facilities, e.g.: the Laser Interferometer Gravitational-Wave Observatory (LIGO) [4] and the Large Hadron Collider (LHC) [5]. But especially in the way we process and store information, relevant to all aforementioned fields, a fundamental change took place. Today's economy is more than ever organized around knowledge and information, rather than industrial goods, and the way our society stores and communicates information is evolving rapidly. Consequently, the current period of human history is described as the information age [6, 7].

The transition to the information age is accompanied by, and to a large extent enabled by, the digital revolution. The world's technological capacity to store, communicate, and compute information shifted from analog to digital electronics within just twenty years and follows an exponential growth. In 1986 only $1 \%$ of the information was stored digitally, but in 2007 an astonishing $94 \%$ were already available on digital media [8]. The impact on the society and our everyday life is enormous: in less than one generation's time we got used to share information, for example via instant messaging or the World Wide 
Web, almost independently of our whereabouts and distances the information needs to travel around the globe.

The foundation to store and process such amount of data are integrated circuits or microchips. As such, this technology needs to advance with the exponential growth of information and the society's expectations of ever faster, smaller and yet more powerful electronic devices. Both trends, the exponential growth of the capacity of microchips and the impact of the technology on our society, were sketched in 1965 by Gordon Moore [9], the co-founder of the Intel Corporation (today's largest semiconductor company). Just seven years after the first realization of a microchip by Jack Kilby and Robert Noyce [7] he envisioned handheld electronic devices and home computers as part of everyday life (see Fig. 1.1). It took about five decades for this vision to become reality as we can easily convince ourselves today by browsing the latest news on our smartphones.

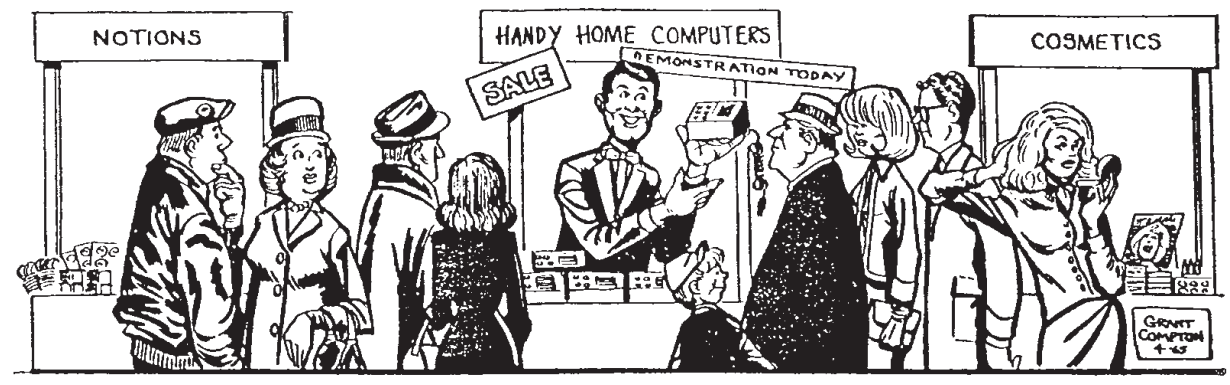

Figure 1.1: An everyday scene sketched in 1965 by Moore [9].

Moore's prediction of exponential growth for the number of transistors per unit area that can be integrated in a microchip is well-known as Moore's law. Although it is not a natural law but an extrapolation based on five data points, it proved accurate for several decades and underlines the ever-growing importance of the microchip technology. Our society got used to such rapid progress and its benefits, setting high expectations for future developments. Not surprisingly, Moore's law has been used in the semiconductor industry to guide long-term planning and to set targets for the next generation of microchip manufacturing.

Progress in semiconductor manufacturing is all about reducing the size of the features that make up the microchip designs. Smaller features allow for faster and more advanced microchips that consume less power and can be produced at lower cost [10]. This progress is enabled by a continuous evolution of the technology to manufacture microchips: the leading candidate for the next generation is extreme ultraviolet (EUV) nanolithography [10, 11]. 


\subsection{EUV Nanolithography}

Lithography is a method of surface printing that was invented by Alois Senefelder (Fig. 1.2) in the eighteenth century as a costeffective method to reproduce text or art work. The name lithography is a combination of the two ancient greek terms lithos, meaning stone, and graphein, which means to write. In this method, a drawing may be produced either by the artist directly upon stone, or it may be made upon paper, and transferred and affixed to the stone. Thereby, the stone is partially protected during the subsequent etching process where a chemical treatment creates a hydrophilic surface, that is a waterattracting surface, at areas that are not covered by the drawing. An oil-based ink can

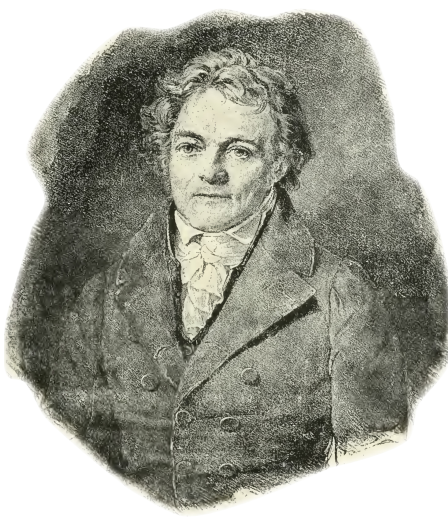

Figure 1.2: A portrait of Alois Senefelder, reproduced from Pennell and Pennell [12]. then be applied and, as it sticks only to the hydrophobic, i.e. water-repellent, part of the drawing, transferred to a blank sheet of paper [12]. Lithography allows for autographic reproductions of the original including smallest features in a fast and cost-effective way.

The current technique for the production of microchips with a cost-effective throughput is photolithography, a method that combines Senefelder's idea of lithography with the principles of photography. The artwork of reproduction is the designed pattern of the microchip called photomask or reticle, which would be the modern equivalent to the stone in traditional lithography. Exposing the photomask with light projects an image of the pattern on the silicon wafer and, thereby, transfers the photomask. A series of chemical treatments, that is the etching process, then either engraves the pattern into the wafer, or even enables deposition of a new material on the pattern. A repetition of this photolithographic cycle allows to build microchips based on two-dimensional patterns. In contrast to traditional lithography, the artwork is not directly reproduced but the microchip is assembled in multiple layers. However, the original idea of transferring complex two-dimensional patterns in a single step allows for a cost-effective mass production.

The smallest length scale that can be assembled today by photolithography, also known as the critical dimension $C D$, is of the order of a few nanometers giving the method its name nanolithography. The history of photolithography is a continuous effort to decrease $C D$ [10] and continues to follow Moore's law. One key physical limit for $C D$ is set by the photographic imaging step and 
scales according to the Rayleigh criterion [13] as

$$
C D \sim \frac{\lambda}{N A}
$$

where $\lambda$ is the wavelength of the light and $N A$ is the numerical aperture that characterizes the resolving power of the optical system used for the projection of the photomask onto the silicon wafer. The evolution of the technology in terms of $\lambda$ is shown in Fig. 1.3 with EUV nanolithography as the latest step of the development. The wavelength is decreased to $\lambda=13.5 \mathrm{~nm}$, an improvement by more than one order of magnitude compared to current state-of-the-art lithography machines that operate at $\lambda=193 \mathrm{~nm}$.

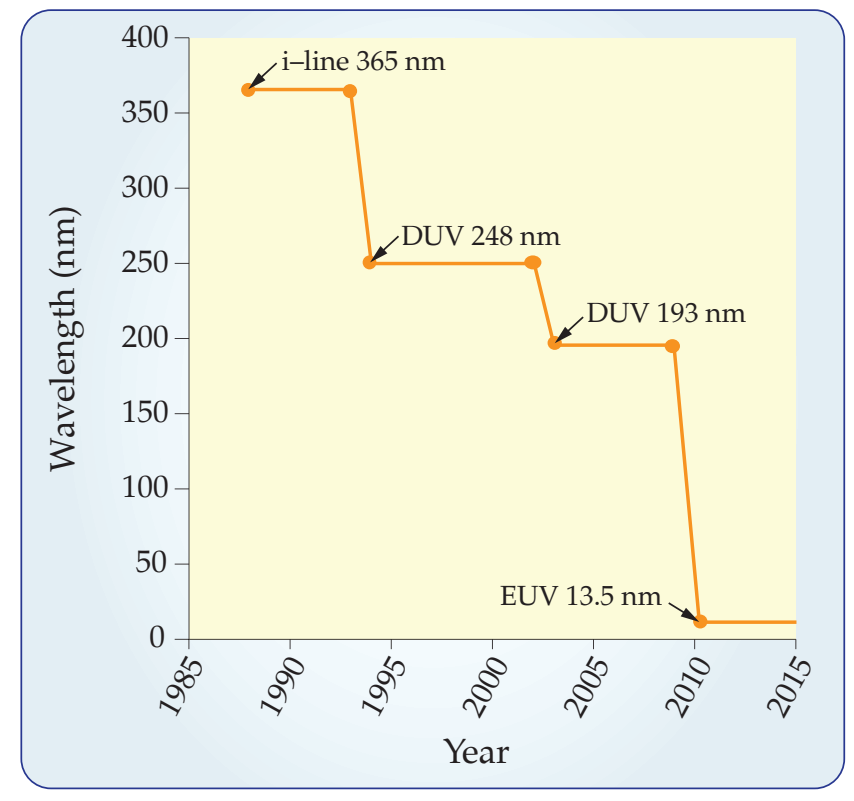

Figure 1.3: Since the mid-1980s, the wavelength of light used in lithography systems has reduced by almost half from $365 \mathrm{~nm}$ to $193 \mathrm{~nm}$ in the deep ultraviolet (DUV). The switch to EUV nanolithography involves a further reduction in wavelength by another factor of almost 15 (reproduced from Wagner and Harned [10]).

EUV nanolithography requires a complete redesign of the lithography machines since the technology departs from any previous technique in a key aspect: all matter, including air or any known material available for optical lenses, absorbs EUV radiation. Consequently, all the optical elements responsible for the imaging step must use reflective lenses, that is mirrors, rather than refractive lenses [10,14]. Furthermore, the entire optical path from the light source to the wafer must be in a near-vacuum (see Fig. 1.4). This 


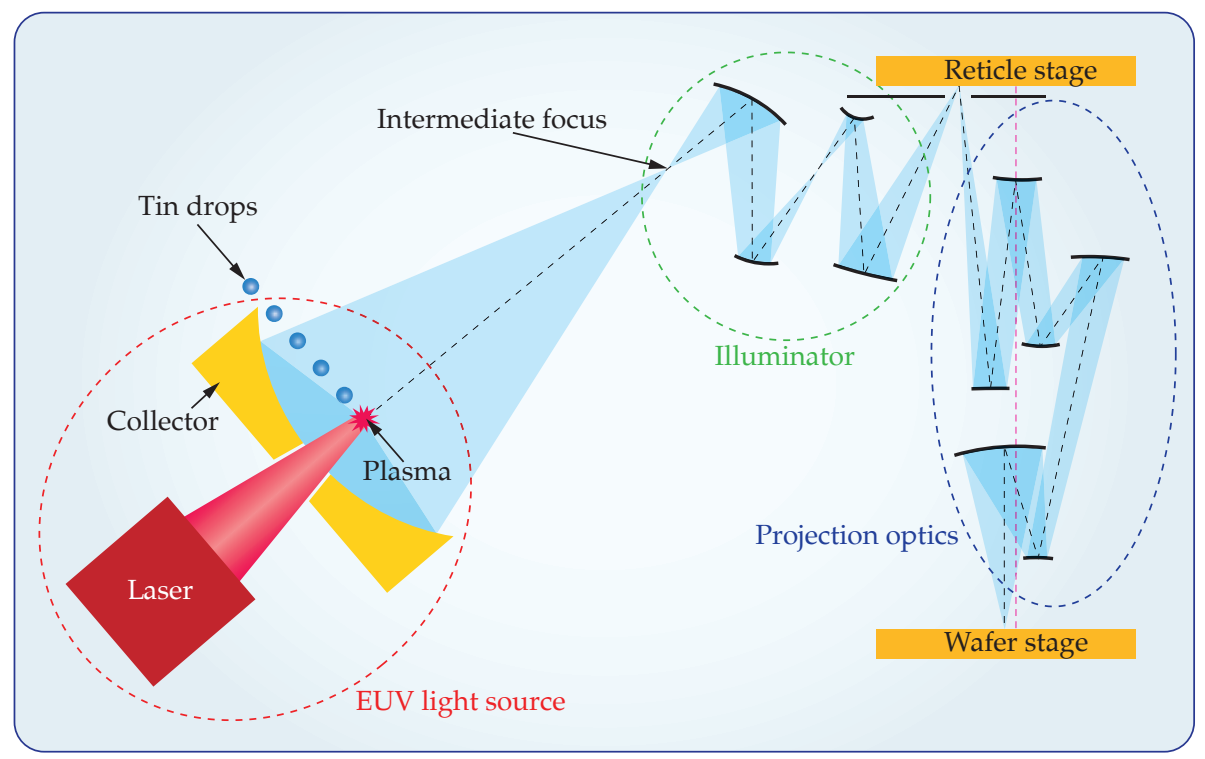

Figure 1.4: A sketch of a nanolithography system including the EUV light source based on a laser-produced plasma of tin. Because all matter absorbs EUV radiation, the optics for collecting the light (collector), conditioning the beam (illuminator) and pattern transfer (projection optics) must use mirrors, and the entire optical path must be housed in a near-vacuum environment (adapted from Wagner and Harned [10]).

renders previous technologies to optimize $N A$, such as immersion techniques where the gap between the final lens and the wafer surface is filled with a liquid medium that has a refractive index greater than one, inapplicable in EUV nanolithography. Nevertheless, the combined effects of $N A$ and the major decrease in $\lambda$ lead to a significant advantage in microchip manufacturing [11], that is a further reduction of $C D$.

One of the major challenges in EUV nanolithography is to create a sufficiently high-power EUV light source. Otherwise, the high throughput in microchip manufacturing for the current and future needs of our society cannot be met. As extensive research has shown the best conversion efficiency, that is the ratio of useable EUV energy extracted in the chosen bandwidth for $\lambda$ to the energy input, is achieved by sources based on tin plasmas [10].

ASML, the leading manufacturer of lithography systems and co-funder of the research program for this thesis, develops laser-produced plasma sources. In this design (Fig. 1.4), micrometer-sized high-speed-jetted liquid tin drops are ionized by high-intensity laser radiation in a vacuum environment. The resulting hot plasma emits EUV radiation, which is collected by a mirror and guided through a dedicated optical system to project the photomask onto the 
silicon wafer. It is beneficial to apply a dual-pulse sequence [15-17]: the first laser pulse carefully shapes the drop into a larger and thinner sheet that is a suitable target for plasma generation. The second, much more energetic, main pulse leads to optical breakdown and the creation of a high-density and high-temperature plasma $[15,18,19]$. Line emission from excited tin ions then provides the actual EUV light $[15,18]$.

\subsection{Fluid dynamics in EUV light sources}

To make the EUV light source (see Fig. 1.4) operational, several major fluiddynamic obstacles have to be overcome. These obstacles involve the shaping of the tin drops into suitable targets for plasma generation, the fragmentation of the tin targets by the laser pulses, the interaction of liquid tin with plasma, and the impact and splashing of tiny tin drops on the surrounding environment and the mirrors. It is an interdisciplinary challenge that combines fluid dynamics, plasma physics, and surface chemistry.

The focus of this thesis is on the fluid dynamics involved in the laser impact on tin drops that are shaped into suitable targets for plasma generation. The shape, position, and stability of the liquid-tin targets directly affect the conversion efficiency of the EUV light source. The detailed understanding of the fluid-dynamic response of the liquid drop to the laser impact poses two fundamental challenges. First, one needs to resolve how momentum is transferred from the laser to the drop. Second, the subsequent deformation dynamics, often accompanied by fragmentation of the deforming drop, have to be quantified.

The application for research on laser-induced fluid motion is not limited to microchip manufacturing, but is of great importance for a broad range of applications. The laser impact leads to a localized forcing, both in time and space, that drives the fluid dynamics. A well-known application is found in non-invasive surgery [20], e.g. in refractive eye surgery. During such an operation, the cornea of a human eye is reshaped by laser pulses to eliminate the patient's dependency on glasses or contact lenses. A novel technique combining a laser-induced phase change and fluid motion is found in the threedimensional printing of metals by laser-induced forward transfer (LIFT) [21]: the laser impact first melts a solid material locally, i.e. induces a phase change, and then transfers it to a receiver substrate. The preparation of liquid and solid samples for mass spectroscopy by laser-induced desorption or ionization [22, 23] are examples where the phase change itself is of primary interest and the induced motion is a secondary effect. Similarly, in pulsed laser deposition [24], the vaporization of the target is the primary goal to transfer the material of 
interest, including liquids [25], to a thin film on a substrate. In summary, the laser impact on flying drops, i.e. free-falling and unconfined drops, combines fundamental research and direct applications.

When the drop is hit by a laser pulse with sufficient energy a part of the liquid tin drop is converted into a plasma state. This laser-induced phase change is the driving force of the fluid dynamics and has been studied before: the complete vaporization or even explosion of micrometer-sized drops can result from the linear absorption of laser energy [26-28]. Self-focusing and dielectric breakdown may lead to plasma formation in transparent drops [2932]. Laser impact has also been used to generate liquid motion by vaporization or plasma formation in confined geometries [33-35], sessile drops [36], and biological matter [20,37-39]. However, the controlled deformation of a freefalling liquid drop by the impact of a laser pulse is unexplored.

A concept widely used in fluid dynamics shall also be used here to put the laser impact on a drop into perspective: a dimensional analysis according to the Buckingham- $\pi$-theorem $[40,41]$ leads to three dimensionless parameters* The Reynolds number

$$
\operatorname{Re}=\frac{U R_{0}}{v}
$$

with $\rho$ as the liquid density, $v$ the liquid kinematic viscosity and $U$ the typical velocity imparted to the drop of initial radius $R_{0}$ by the laser impact. This parameter compares the fluid inertia to viscous forces. Similarly, the Weber number

$$
\mathrm{We}=\frac{\rho U^{2} R_{0}}{\gamma}
$$

compares the forces due to inertia and surface tension $\gamma$. Both parameters, $\mathrm{Re}$ and We, can be combined to the Ohnesorge number $\mathrm{Oh}=\sqrt{\mathrm{We}} / \mathrm{Re}=$ $v / \sqrt{\gamma R_{0} / \rho}$, which compares viscous to surface forces. The third independent parameter can be expressed as an energy ratio,

$$
N=\frac{\rho U^{2} R_{0}^{3}}{E_{\mathrm{L}}}
$$

and relates the kinetic energy of the drop to the laser-pulse energy $E_{\mathrm{L}}$. It takes into account that in our problem the driving force originates from a laser-matter interaction that is represented here in a general way by $E_{\mathrm{L}}$. An important part of this thesis is to reveal in detail how the laser energy is converted to kinetic energy of the drop.

*Taking into account $n=6$ physical variables such as fluid density $\rho$, surface tension $\gamma$, kinematic viscosity $v$, the initial drop radius $R_{0}$ and the laser-pulse energy $E_{\mathrm{L}}$ that induces a characteristic fluid velocity $U$ leads to $k=3$ physical dimensions and $p=n-k=3$ dimensionless parameters. 
Table 1.1 gives an overview for the laser impact on a drop in terms of its dimensionless parameters. Next to the tin drops as encountered in the EUV light source the corresponding values for a water experiment with closely matching Weber and Reynolds numbers are given. Remarkably, the length scale of the water experiment, expressed by $R_{0}$, is increased by almost two orders of magnitude in comparison to the tin drops. The dimensional analysis identifies an opportunity for our research: an upscaled experiment shows the same fluid-dynamic response to the laser impact but is much easier to handle experimentally due to the increase in length scale and availability of liquid water at room temperature. This fact motivates an integral part of this thesis: the design, planning, and construction of an experimental setup that is based on water drops and dedicated to the fluid dynamics. The length scale is chosen such that a variety of visualization techniques at improved temporal and spatial resolution as compared to the tin drops can be applied to the laser impact on a drop.

The dimensional analysis characterizes the fluid-dynamic response in a general way, independent of the actual experimental realization and the particular choice of the liquid or length scale. As a consequence, results obtained from the water experiments can be transferred to tin and vice versa. Furthermore, one may draw analogies between features of previous studies and the laser impact on a drop. An important analogy is given in Fig. 1.5 that shall guide the reader in this thesis: the mechanical impact of a drop on a

Table 1.1: Parameters for tin drops under typical EUV source conditions taken from $\S 5$ and water drops with similar dimensionless parameters. For both liquid systems, micrometer-sized tin drops and millimeter-sized water drops, viscous effects can be neglected $(\mathrm{Oh} \ll 1)$ and the driving mechanism is energetically inefficient $(N \ll 1)$.

\begin{tabular}{llcc}
\hline parameter & unit & tin & water \\
\hline$\rho$ & liquid density $\left(\mathrm{kg} / \mathrm{m}^{3}\right)$ & 6968 & 998 \\
$\gamma$ & surface tension $(\mathrm{N} / \mathrm{m})$ & 0.544 & 0.072 \\
$\nu$ & liquid kinematic viscosity $\left(\mathrm{m}^{2} / \mathrm{s}\right)$ & $0.27 \times 10^{-6}$ & $10^{-6}$ \\
$R_{0}$ & initial drop radius $(\mathrm{m})$ & $25 \times 10^{-6}$ & $10^{-3}$ \\
$U$ & drop velocity $(\mathrm{m} / \mathrm{s})$ & 100 & 10 \\
$E_{\mathrm{L}}$ & laser-pulse energy $(\mathrm{J})$ & $10^{-2}$ & $10^{-1}$ \\
\hline $\mathrm{We}$ & Weber number & 3200 & 1400 \\
$\mathrm{Re}$ & Reynolds number & 9300 & 10000 \\
$\mathrm{Oh}$ & Ohnesorge number & $6 \times 10^{-3}$ & $3 \times 10^{-3}$ \\
$N$ & modified Stuart number & $10^{-4}$ & $10^{-3}$ \\
\hline
\end{tabular}



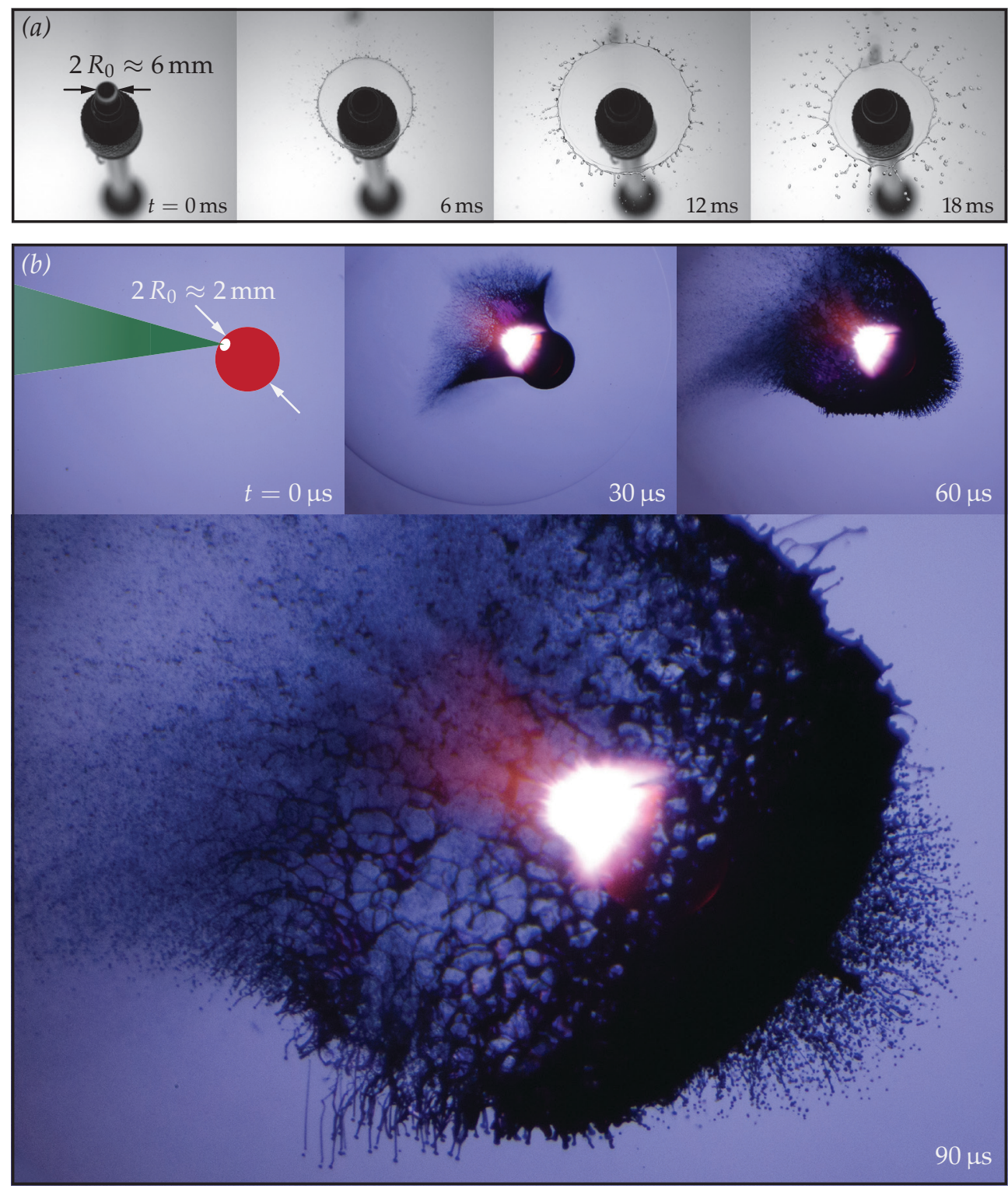

Figure 1.5: Deformation and fragmentation of a drop upon impact. (a) An ethanol drop falls down under the influence of gravity and impacts on a mechanical pillar. The sequence is viewed from the top (adapted from Villermaux and Bossa [42]). (b) A focused laser pulse hits a magenta-dyed drop that is falling from top to bottom of the image at very low speed. The laser impact leads to optical breakdown in the water and the resulting plasma emits a white glow and drives the fluid dynamics. The sequence is viewed from the side, perpendicular to the direction of the laser-pulse propagation (taken from $\S 3$ ). 
pillar [42] is compared to the laser impact on a flying drop. The impulsive acceleration of the drop due to the laser impact is similar to the impulsive stop of a drop impacting on a pillar. Both experiments share common features as the drop is initially a sphere, deforms due to the impact and finally breaks up in tiny fragments. The scale of the experiment, the driving mechanism of the fluid dynamics and especially the timescales vary between the two cases. However, the most important dimensionless parameter, the Weber number, is of the same order.

Results obtained for the deformation dynamics of a drop upon mechanical impact and ideas developed for the analytical treatment of the fragmentation shall guide the analysis in this thesis. The impact of drops onto a solid substrate has been studied thoroughly (see e.g. Ref. [42-48]). However, no consensus on the deformation dynamics has yet been reached. The fragmentation of drops, liquid sheets, and ligaments has been studied in a wide context $[49,50]$. In these studies, the fragmentation was caused by, for example, hole nucleation by bursting bubbles [51, 52], shear instabilities with the surrounding medium [53-55], or impact upon an object $[42,56]$. By contrast, the fragmentation of a liquid drop induced by a laser pulse has not been studied before. The goal of this thesis is to describe and understand the life of the drop: starting with the laser impact, followed by the deformation of the drop and how it depends on the laser-pulse properties up to the final breakup of the liquid body into tiny fragments.

\subsection{A guide through the thesis}

In $\S 2$ a brief introduction to the laser impact on a drop based on the water experiment is given. The details of the experimental setup, the design of which is an integral part of this thesis, are presented in $\S 3$. The remaining chapters follow the chronology of the drop impact. First, the driving mechanism for water and tin experiments is studied in $\S 4$ and $\S 5$, respectively. The induced drop deformation and how it depends on the laser-pulse properties is described in $\S 6$. All of this lays the foundation to understand the breakup of the drop presented in $\S 7$. Finally, $\S 8$ condenses the thesis in an overall conclusion and gives an outlook. 


\section{2 \\ Laser impact on a drop*}

Our contribution to the Gallery of Fluid Motion (Fig. 2.1) received the Milton-vanDyke award in 2014 for showing that the response of a liquid drop to the impact of a nanosecond laser-pulse is violent: the drop gets strongly deformed and propelled forward at several $\mathrm{m} / \mathrm{s}$, and subsequently breaks up or even explodes. Detailed understanding of this process is of key importance for the generation of extreme ultraviolet (EUV) light in the latest nanolithography machines. For the video, we recorded the drop's response to various laser-impact conditions by high-speed imaging at 20000 frames per second (FPS) and by stroboscopic illumination at an effective frame rate of 10 million FPS. We present a scaling law and compare experimental results to boundary-integral simulations, in order to elucidate how the drop is propelled and deformed.

Figure 2.1: Scan the quick response code with your smartphone to watch the Milton-van-Dyke winner of the Gallery at Fluid Motion 2014 at https : / www . youtube. com/watch?v=bRbHDtPbHeO.

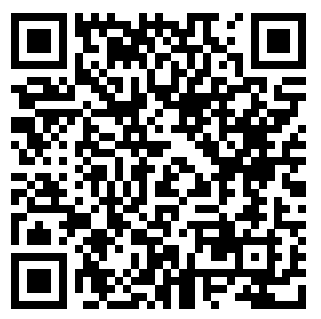

${ }^{*}$ Invited publication for the Milton-van-Dyke award of the Gallery of Fluid Motion 2014: Alexander L. Klein, Claas Willem Visser, Wilco Bouwhuis, Henri Lhuissier, Chao Sun, Jacco H. Snoeijer, Emmanuel Villermaux, Detlef Lohse, and Hanneke Gelderblom. Laser impact on a drop. Physics of Fluids, 27(9):091106, 2015. 
The impact of a laser pulse on a highly-absorbing liquid drop can lead to a violent response: the drop is accelerated, strongly deforms, and eventually fragments. Shock waves, the ejection of matter, and even plasma formation can accompany this process (see Fig. 2.2).

The total energy absorbed by the drop and its spatial distribution in the superficial layer determine the hydrodynamic response to the laser impact. For a localized energy deposition the threshold of optical breakdown in water is exceeded, which provokes a violent drop explosion (Fig. 2.2 (a)). When the energy density in the superficial layer is decreased, plasma generation is avoided and a fluorescence effect shifting the green laser light to yellow becomes visible (Fig. 2.2 (b, c)). Momentum conservation for the ablated mass due to the vaporization yields a scaling law for the propulsion speed of the drop

$$
U \sim \frac{E_{\mathrm{abs}}-E_{\mathrm{th}}}{\rho R_{0}^{3} \Delta H} u .
$$

This speed scales linearly with the absorbed laser energy $E_{\text {abs }}$ beyond the threshold energy $E_{\text {th }}$ needed to heat the liquid layer to the boiling point, where $\Delta H$ is the latent heat of vaporization and $u$ the thermal speed of the ablated mass in vapor state. The derivation can be found in $\S 4$ and an extension to plasma-driven drops is presented in $\S 5$.

The deformation of the drop changes with the spatial distribution of the absorbed energy: from a strongly curved thin liquid sheet in Fig. 2.2 (b) to a flatter liquid sheet in Fig. 2.2 (c). A quantitative analysis of this relation is given in $\S 6$. The deformation occurs on the inertial timescale $\tau_{i}=R_{0} / U \sim 10^{-4} \mathrm{~s}$, and is eventually slowed down by surface tension $\gamma$ on the capillary timescale $\tau_{c}=\left(\rho R_{0}^{3} / \gamma\right)^{1 / 2} \sim 10^{-3}$ s (see Fig. 2.3). Both timescales are clearly separated from those of the laser-matter interaction, namely, the laser pulse duration $\tau_{p}=10^{-8} \mathrm{~s}$ and the duration of the ejection of matter $\tau_{e} \sim 10^{-5} \mathrm{~s}$. The timescales relevant to the laser impact on a drop can thus be ordered:

$$
\tau_{p} \ll \tau_{e} \ll \tau_{i}<\tau_{c} .
$$

The separation of timescales requires an elaborate combination of stroboscopic (Fig. 2.2) and high-speed (Fig. 2.3 (a)) imaging techniques to resolve the physical phenomena experimentally, see $\S 3$. However, it eases the modeling of the fluid dynamics: the complex laser-matter interaction can be replaced by an appropriate pressure pulse acting on the spherical drop. The last example in Fig. 2.3 (b) makes use of this modeling strategy and shows the comparison between experiments and boundary-integral (BI) simulations for the deformation of the drop perpendicular to the laser pulse. 

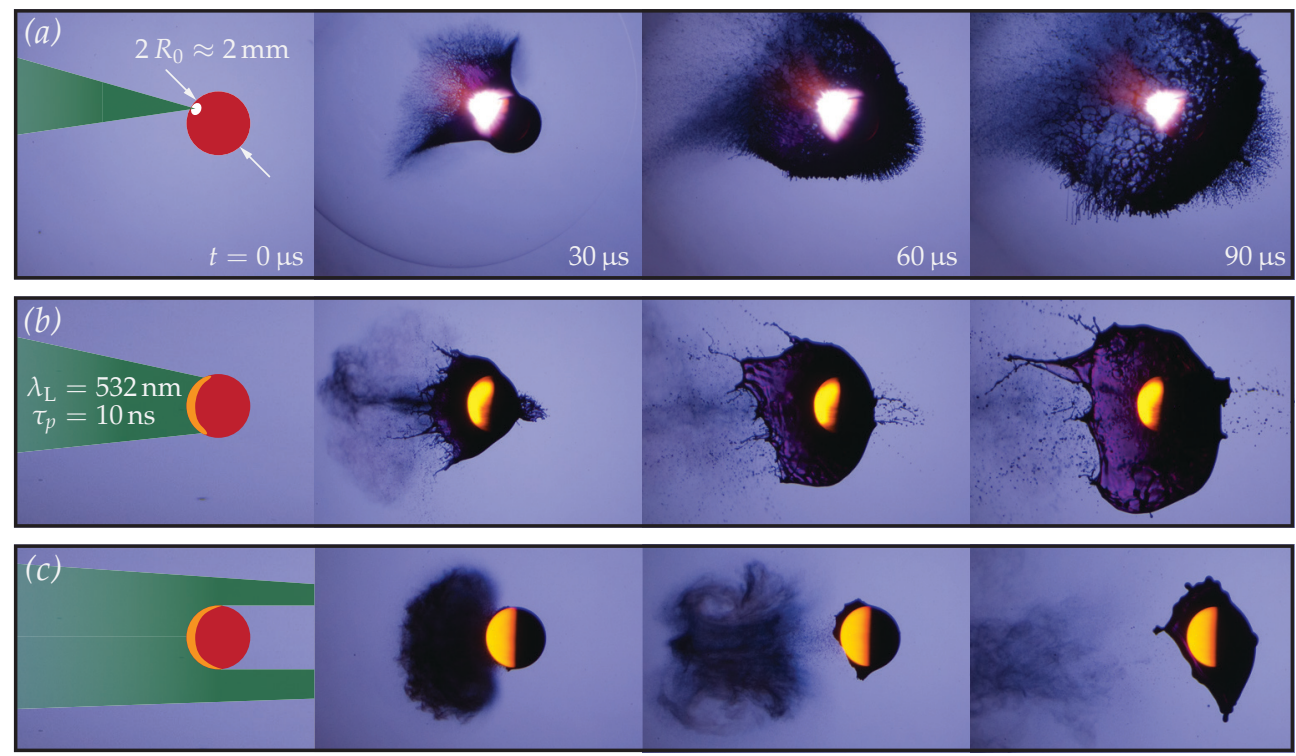

Figure 2.2: Laser pulses of duration $\tau_{p}$, wavelength $\lambda_{\mathrm{L}}$ and constant total energy impacting from the left on magenta-dyed water drops with an initial radius $R_{0}=$ $0.9 \mathrm{~mm}$. The dye limits the penetration depth of the laser light $\delta$ to a superficial layer of the drop, i.e. $\delta / R_{0} \ll 1$. The density $\rho$ and surface tension $\gamma$ of the liquid are unaffected by the dye (see §3.7). Images are taken $30 \mu \mathrm{s}, 60 \mu \mathrm{s}$, and $90 \mu$ s after impact with a color camera and stroboscopic backlight illumination. The sketches in the first column illustrate the respective optical arrangements: (a) Tightly focused laser beam leading to a white plasma glow and a violent ablation from the drop. A spherical shockwave emitted from the location of laser impact is visible at $t=30 \mu \mathrm{s}$. (b) Moderately focused laser beam resulting in a strongly curved liquid sheet. (c) Uniform laser irradiation ablating the drop surface by a local vaporization and ejecting a cloud of mist in opposite direction to the laser beam. Note that the laser energy actually absorbed by the drop varies between (a), (b), and (c). The shutter of the camera is open during the whole experiment to capture both, the light emitted by the plasma or fluorescent effect just after laser impact and the hydrodynamic response of the drop. See $\S 3$ for more details on the experimental setup. 
(a)
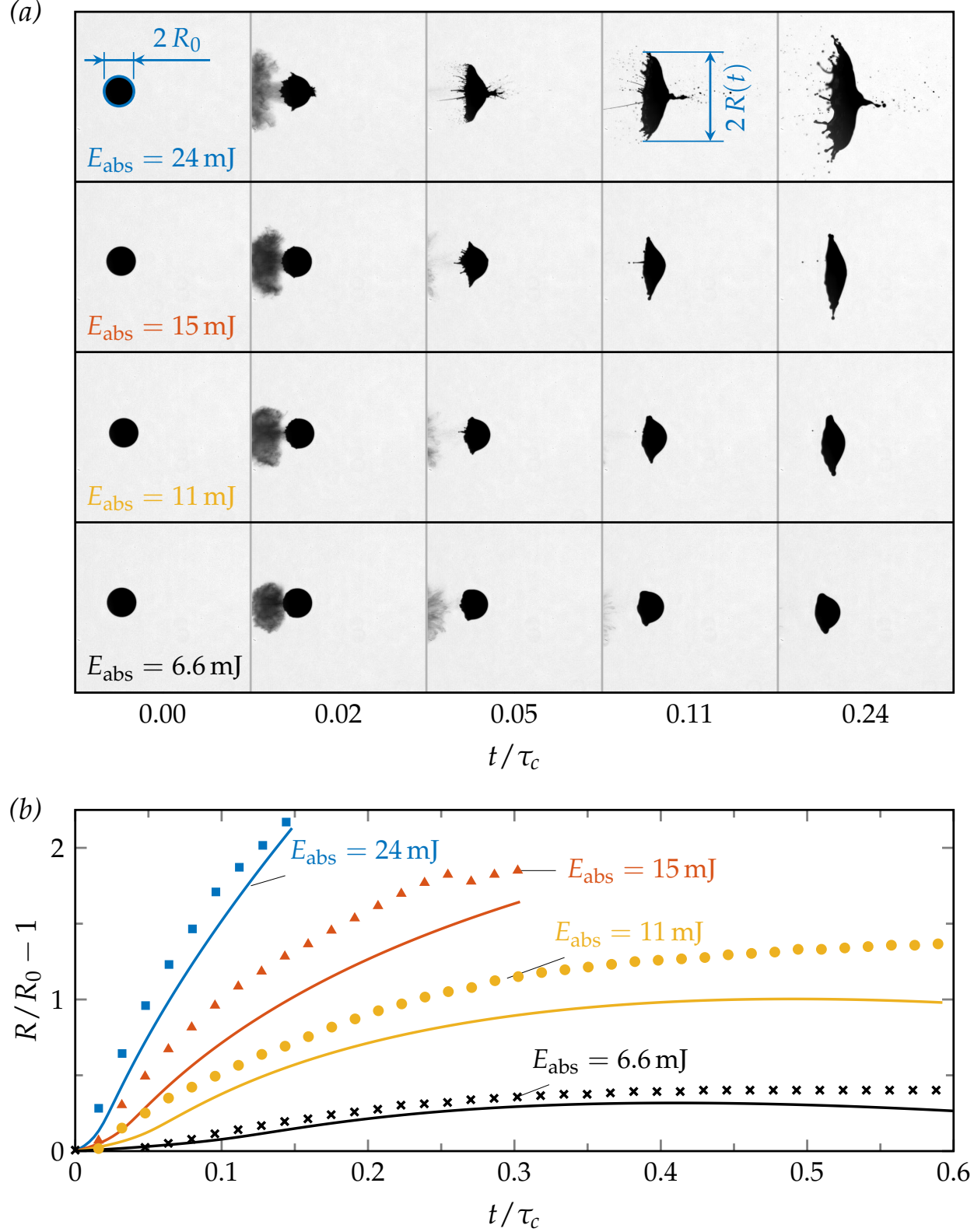

Figure 2.3: Drop deformation dynamics for an uniform laser irradiation (configuration Fig. 2.2 (c)) at different laser-pulse energies. (a) High-speed images for a dyed water drop with initial radius $R_{0}=0.9 \mathrm{~mm}$ hit by a laser pulse at $t=0 \mathrm{~s}$. $E_{\mathrm{abs}}$ is the energy that is absorbed by the drop (increasing from bottom to top). (b) Comparison between experiments (markers) and BI simulations (solid lines) for the radial expansion of the drop at different laser energies. The simulations are parameter-free, that is all parameters are determined a priori, which is explained in $\S 4$ and $\S 6$. 


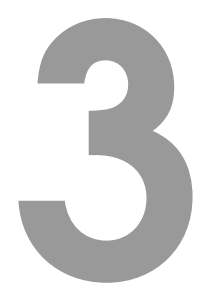

\section{Experimental setup*}

We present an apparatus to control and visualize the response of a liquid target to a laser-induced vaporization. A millimeter-sized drop serves as target and we present two liquid-dye solutions that allow a variation of the absorption coefficient of the laser light in the drop by seven orders of magnitude. The excitation source is a $Q$-switched Nd:YAG laser at its frequency-doubled wavelength emitting nanosecond pulses with energies above the local vaporization threshold. Absorption of the laser energy leads to large-scale fluid motion at timescales that are separated by several orders of magnitude, which we spatiotemporally resolve by a combination of ultra high-speed and stroboscopic high-resolution imaging in two orthogonal views. Surprisingly, the large-scale fluid motion upon laser impact is completely controlled by the spatial energy distribution obtained by a precise beam-shaping technique. The apparatus demonstrates the potential for accurate and quantitative studies of laser-matter interactions.

${ }^{*}$ To be submitted as: Alexander L. Klein, Detlef Lohse, Michel Versluis, and Hanneke Gelderblom. Apparatus to control and visualize the impact of a high-energy laser pulse on a liquid target. 


\subsection{Introduction}

Light moves liquid matter in various ways. The direct interaction of an electromagnetic wave with a liquid surface exerts a pressure on the interface that may move the liquid: photons exchange momentum with the liquid as the light path changes at the interface between two media of different refractive index, an effect described as optical radiation pressure [57]. The observed motion is normally small [58] unless competing forces such as capillary forces are weakened, for example under near-critical conditions when surface tension vanishes [59]. Intense field strengths are required to exert the radiation pressure onto the liquid target, and therefore focused laser light is used. Laser radiation can also induce thermocapillary forces, which can be applied to control liquids. The localized heating by a laser, either directly by linear absorption of the light in the liquid sample or indirectly by heating the substrate the liquid sample is in contact with, introduces thermocapillary stresses $[60,61]$ that drive the fluid dynamics. This effect of induced fluid motion by thermocapillary stresses is well-known as the Marangoni effect [62].

A laser-induced phase change is another way to move liquids by optical radiation and it allows for large deformations and flow speeds to be reached, see Fig. 3.1. Here, the local field strength or energy density must be high enough to supply the energy required for the phase change, such as a vaporization or plasma generation. Local vaporization is achieved by linear absorption of the laser energy in the liquid, or by additives, e.g. dyes or dyed particles [26-28, 33, 34, 63]. Self-focusing and a dielectric breakdown may lead to plasma formation in otherwise transparent liquids [29-32, 35, 36]. Both effects are for example important in biological matter [20,39] as encountered in medical applications. An application where the material is initially solid and needs to melt, i.e. the liquid phase is an intermediate step, is found in the laser-induced forward transfer of metals [21]. The preparation of liquid and solid samples for mass spectroscopy by laser-induced desorption or ionization $[22,23]$ are examples where the phase change itself is of primary interest and the induced motion is a secondary effect. Similarly, in pulsed laser deposition [24] the vaporization is the primary goal to transfer the material of interest, including liquids [25], to a thin film on a substrate.

The setup presented here is motivated by the physical processes found in laser-produced plasma light sources for extreme ultraviolet (EUV) nanolithography $[11,15,17]$. In these sources a liquid tin drop is impacted by a first laser pulse to shape the drop into a suitable target for plasma generation. A second pulse creates the plasma, where the line emission from excited tin ions provides the EUV light $[15,18]$. Our setup can be understood as large-scale model system to study the fluid dynamics of the target formation by the first laser 

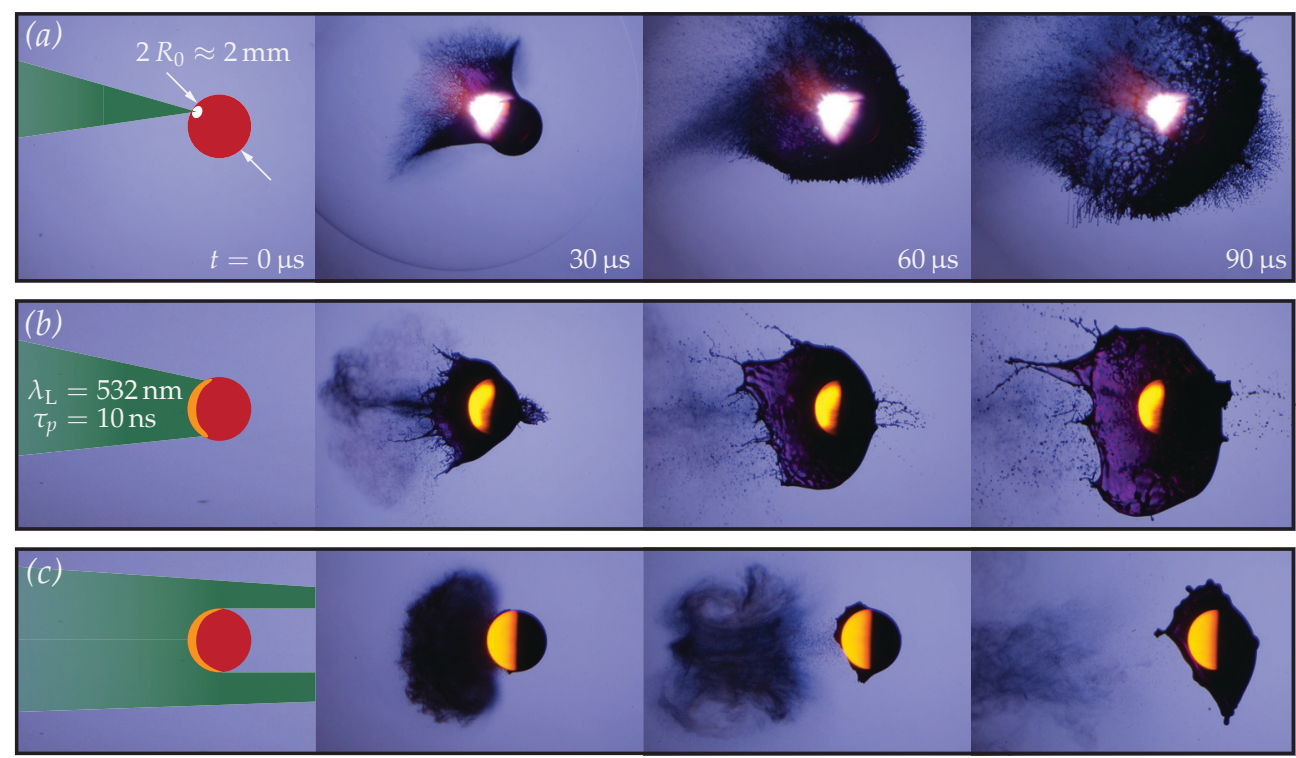

Figure 3.1: Side-view images taken stroboscopically with a color camera (Nikon D5100) and a broadband pulsed light source (NANOLITE KL-K by HSPS) for three laser focusing conditions (illustrated by a sketch in the left column): (a) Tightly focused laser beam leading to a white plasma glow and a violent ablation from the drop. A spherical shockwave is visible at $t=30 \mu \mathrm{s}$. (b) Moderately focused laser beam resulting in a strongly curved liquid sheet. (c) Uniform laser irradiation ablating the drop surface and ejecting a cloud of mist in opposite direction to the laser beam. Note that the laser energy actually absorbed by the drop varies between (a), (b), and (c). The liquid used in the experiments is an aqueous magenta-colored ink (IJC-5920 by Sensient Imaging Technologies). Images are taken from §2, where a laser with $\tau_{p}=10 \mathrm{~ns}$ is used as opposed to $\tau_{p}=5 \mathrm{~ns}$ used in the remainder of this chapter.

impact. However, the features and capabilities of the setup described here can be used to study laser-matter interactions and laser-induced liquid motion for a much broader range of experiments: the target, in our case a free-falling spherical drop, may be replaced with planar geometry such as a liquid film to study the laser-induced forward motion, for example for non-Newtonian liquids or viscoelastic hydrogels. In general, the laser impact upon a liquid target results in a forcing, concentrated both in time and space, and can be visualized in our setup. This allows for accurate and quantitative studies of laser-matter interactions that induce fluid motion.

We present in $\S 3.2$ to $\S 3.8$ an experimental setup that not only allows to study but also to control the fluid dynamics driven by a laser-induced vaporization. The scale of the experiment is set by the initial radius $R_{0} \approx 1 \mathrm{~mm}$ of a free-falling drop that is hit by a laser pulse emitted with a duration of $\tau_{p}=5 \mathrm{~ns}$ 
and at a wavelength of $\lambda_{\mathrm{L}}=532 \mathrm{~nm}$. We control the deposition of laser energy in the liquid by adding a dye such that the linear absorption coefficient $\alpha$ at the wavelength $\lambda_{\mathrm{L}}$ can be varied in comparison to $R_{0}$ over several orders of magnitude, i.e. $5 \times 10^{-5} \leq R_{0} \alpha \leq 400$. The spatial distribution of laser energy can further be tuned by the focusing condition of the laser in relation to the drop. We explain in detail how the energy distribution can be visualized and changed, either by a beam-shaping technique or varying $\alpha$. The purpose of our setup is to study the fluid-dynamic response of the liquid target to a laser impact. To this end, the experimental apparatus allows for extensive visualization of the complete process by high-speed and stroboscopic imaging. Having introduced the experimental apparatus, we present a few results of the system in §3.9: the stability of the control parameters of our experiment is discussed, followed by a brief introduction to the laser impact on a drop based on the stroboscopic imaging. Finally, we use the high-speed imaging for experiments on how the fluid dynamics can be controlled by the laser-beam profile. We then give a summary of our work in $\S 3.10$.

\subsection{System overview}

A key feature of the experimental apparatus is the ability to control the laser impact in terms of the laser-pulse energy: both the absolute scale and the spatial distribution of energy can be controlled and visualized. We present two optical configurations to impact the liquid target, which each have their own advantages. First, a free beam propagation is introduced together with the overall layout (Fig. 3.2) of the setup in §3.3. This beam path leaves enough freedom to integrate other components such as additional optics to change the polarization state of the laser beam. In case more control over the spatial distribution of laser energy at the impact location is required we present a second configuration in $\S 3.4$. This optical path incorporates a beam-shaping technique to first modify the beam profile in the near-field of the laser. Then, an imaging technique is applied to propagate the near-field image to the impact location where the drop is placed.

Equally important is the next aspect of our experiment: the visualization of the fluid-dynamic response of the drop to the laser impact. We describe in $\S 3.5$ a combination of high-speed and stroboscopic imaging techniques that are incorporated in the laser-beam path. The response of the drop to the laser impact is then visualized in two orthogonal views: a side-view perpendicular to the laser beam and a back-view that is along the laser-beam propagation.

The spatial scale of the experiment is set by the initial radius $R_{0}$ of the drop, where we chose for a millimeter-sized drop based on two considerations. 
First, the ease to create drops of that size for common liquids as described in §3.6. Second, the timescale of the fluid dynamics, or at least the latetime dynamics, is accessible for high-speed imaging, see §3.5. Furthermore, stroboscopic imaging at this scale allows for recordings with high spatial and temporal resolution. The repetition rate of the experiment is then set to $f_{\exp }=1 \mathrm{~Hz}$, limited by the frame rate of the high-resolution cameras used during stroboscopic recordings.

Preliminary experiments showed that to propel a millimeter-sized drop a laser pulse with an energy of a few $100 \mathrm{~mJ}$ is required. Commonly available at such pulse energies are Nd:YAG lasers with wavelength of $\lambda_{\mathrm{L}}=532 \mathrm{~nm}$. However, to tune the linear absorption of light at that wavelength, i.e. to control the length scale on which the laser energy is absorbed in the liquid, the addition of a dye is necessary for common liquids such as water as explained in §3.7. A high degree of control over the fluid dynamics and laser-impact conditions, required especially for stroboscopic imaging techniques, goes along with a precise control system, which is described in $\S 3.8$.

\subsection{Laser system and free beam propagation}

The layout of the experimental apparatus with a free beam propagation is shown in Fig. 3.2. The main laser used to impact the liquid drop is a Q-switched Nd:YAG laser system (Q-smart 850 by Quantel) at its fundamental frequency. Its output is frequency-doubled in a temperature-stabilized crystal (second harmonic generator (SHG) by Quantel) to generate laser light at a wavelength of $\lambda_{\mathrm{L}}=532 \mathrm{~nm}$ emitted within a pulse duration of $\tau_{p}=5 \mathrm{~ns}$ full width at half maximum (FWHM). The cavity optics and alignment are altered by the manufacturer to meet the design repetition rate of our experiment $f_{\exp }=1 \mathrm{~Hz}$ and delivers a slightly elliptical flat-top beam profile. The laser beam is linearly polarized, which allows for a convenient attenuation by splitting the beam in a polarizing beam splitter (PBS, PBS25-532-HP by Thorlabs or 2-HPCB-B-0254 by Altechna), see also Fig. 3.2. The split-up ratio between the energy redirected into a beam dump and the remaining energy used in our impact experiment is set by a $\lambda / 2$-plate in a motorized rotation mount (K10CR1/M by Thorlabs). That way we can set the energy $E_{\mathrm{L}}$ manually or by software control in the range $E_{\mathrm{L}, \min }=1 \mathrm{~mJ} \leq E_{\mathrm{L}} \leq E_{\mathrm{L}, \max }=420 \mathrm{~mJ}$.

A continuous-wave laser diode (CPS532 by Thorlabs) serves as light source for the alignment laser. It is coupled in the laser-beam path at the same PBS used for the attenuation of the main laser (Fig. 3.2). The beam diameter of the diode laser is increased by a factor of three in a Galilean beam expander [64] $\left(\mathrm{f}_{1}=-50 \mathrm{~mm}, \mathrm{f}_{2}=150 \mathrm{~mm}\right)$ and can be matched precisely to the main laser 
by a diaphragm (see Fig. 3.2). The alignment laser allows safe operation without laser-safety goggles when attenuated sufficiently by a neutral density (ND) filter. The optical path of the main laser is not affected by the optical positioning of the alignment laser. Thereby, the total optical path length can be minimized, which is of particular importance when imaging the near-field as described in $\S 3.4$. In this section, we continue our description of a free beam propagation and general features such as the energy measurement that are also used later in combination with the near-field imaging technique.

The laser energy $E_{\mathrm{L}}$ is measured by the energy meter 1 (energy sensor is a QE12LP-S-MB connected to an energy monitor S-LINK-2 by Gentec ElectroOptics). We use a wedged beam sampler (BSF10-A by Thorlabs) for in-plane polarized light to split-off $1 \%$ by reflection at $45^{\circ}$ incidence. In case we change the polarization state, either to a out-of-plane or a mixed polarization state as will be done for the beam-shaping in $\S 3.4$, we place a wedged window (WW11050-A by Thorlabs) as beam sampler at near-zero incidence to splitoff approximately $0.5 \%$ of laser light, independent of the polarization state in that case. A focusing lens $\mathrm{f}_{\mathrm{EM}}=150 \mathrm{~mm}$ matches the beam diameter to the sensor size of energy meter 1 . For calibration purposes a second energy meter (same type as energy meter 1 but protected from the high-energy beam by an attenuator QED-12 by Gentec Electro-Optics) can be placed directly in the path of the main laser. This way the readout at the energy meter 1 can be calibrated against an arbitrary position along the laser-beam path. In particular, the dimensions of the sensor head allow for a direct placement at $z=0 \mathrm{~mm}$ within the drop-impact chamber (Fig. 3.4) to include any loss of light at optical elements in an appropriate calibration curve.

The laser beam is expanded in a Galilean beam expander $\left(\mathrm{f}_{3}=-250 \mathrm{~mm}\right.$, $\mathrm{f}_{4}=500 \mathrm{~mm}$ ), raised in height in a periscope assembly before it is focused by lens $\mathrm{f}_{5}=400 \mathrm{~mm}$ into the drop-impact chamber (see Fig. 3.2 and 3.4). The beam expansion by a factor of $f_{4} /\left|f_{3}\right|=2$ is required to increase the initial beam diameter $d_{\mathrm{L}}=9 \mathrm{~mm}$ to a size that prevents the local fluence $F$ to exceed the laser-induced damage threshold (LIDT) on any optical element. All optics have appropriate laser-line coatings for $\lambda_{\mathrm{L}}=532 \mathrm{~nm}$ with a typical LIDT of $F_{\text {LIDT }}=5 \mathrm{~J} / \mathrm{cm}^{2}$ leading to a minimum spot size $d_{\min } \geq$ $2\left(E_{\mathrm{L}, \max } /\left(\pi F_{\mathrm{LIDT}}\right)\right)^{0.5}=3.2 \mathrm{~mm}$ for each optical element at maximum laser energy. The periscope assembly allows us to adapt the height of the laser-beam path to about $250 \mathrm{~mm}$ above the optical table to accommodate the high-speed cameras that are placed on translational stages. Before the laser beam enters the chamber a dichroic mirror combines the laser-beam path and the optical path of the back-view visualization, which is explained in more detail in $\S 3.5$.

The diameter $d_{0}$ of the laser beam at the drop location can be adjusted by setting the position of lens $f_{5}$ along the beam-propagation axis $\vec{e}_{z}$. The smallest 


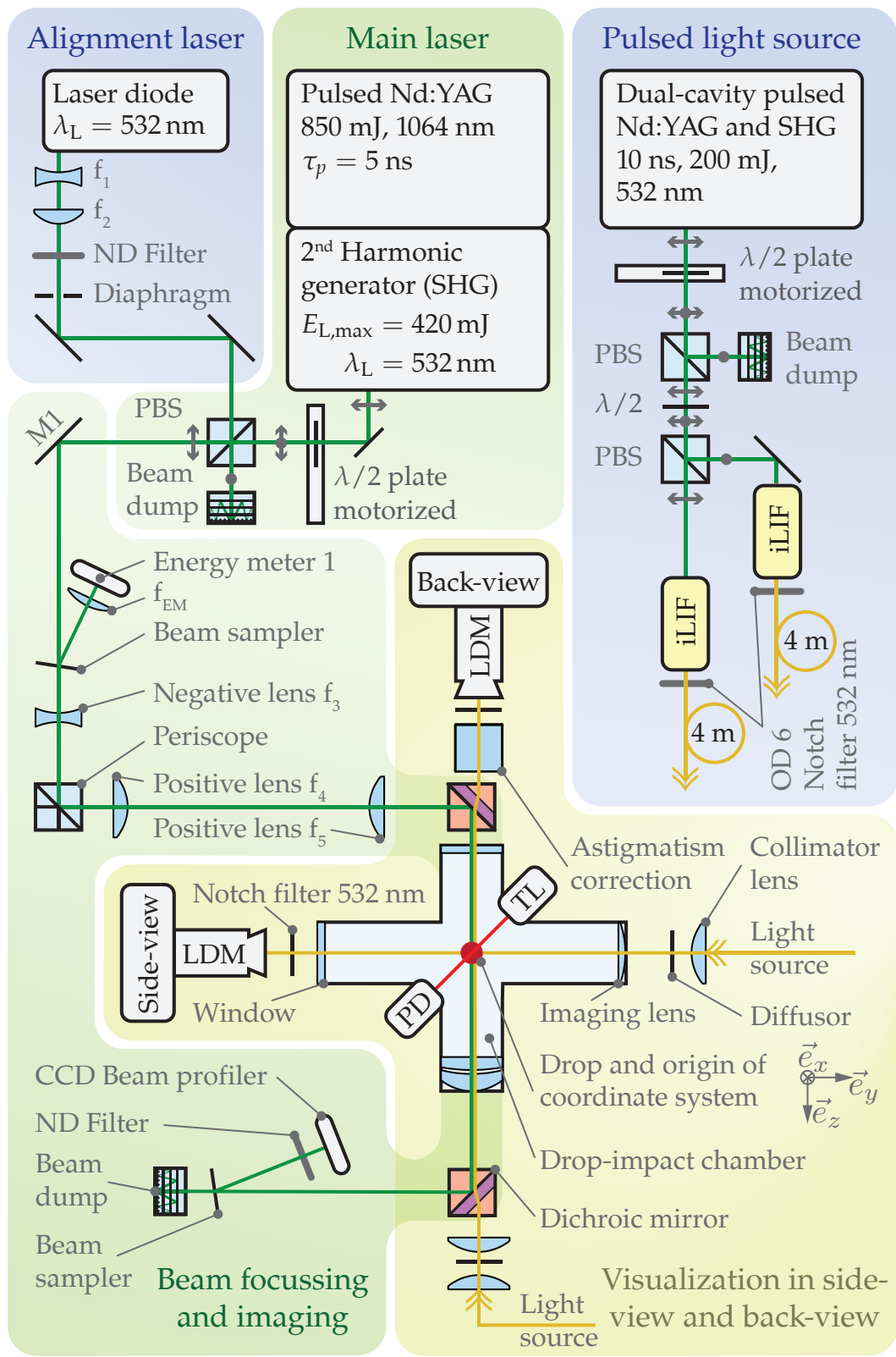

Figure 3.2: Sketch of the optical path of the laser system and visualization as arranged on an optical table with a size of $2.4 \mathrm{~m} \times 1.5 \mathrm{~m}$ (shown not to scale). The symbols on top of the light path indicate out-of-plane $(\bullet)$ and in-plane polarization $(\leftrightarrow)$. Optical mirrors operated at an angle of incident (AOI) of $45^{\circ}$ are represented by black solid lines. The mirror labeled M1 serves as reference point in Fig. 3.3, where an advanced laser-beam path is shown to scale. The drop defines the origin of our coordinate system, where $\vec{e}_{z}$ is aligned to the laser-beam propagation and $\vec{e}_{x}$ to gravity. 
beam diameter at the drop position is set by the beam waist $\omega_{0}$, which is of the order of a few tens of micrometer given our laser system and focusing conditions. This means that we can cover the range $\omega_{0} / R_{0} \approx 0<d_{0} /\left(2 R_{0}\right)<$ 5. In principle, even larger values are possible but out of scope from a fluiddynamics point of view, since the laser fluence is too low in such a case to induce a considerable fluid motion.

Laser light that passes the drop is captured by a lens combination $\mathrm{f}_{6,7}$ to image the drop-impact location onto the charge-coupled device (CCD) of a beam profiler (BC106N-VIS/M by Thorlabs). The combination of a planoconvex lens $\mathrm{f}_{6}=750 \mathrm{~mm}$ and a meniscus lens $\mathrm{f}_{7}=300 \mathrm{~mm}$ to a lens with an effective focal length [65] of $f_{6,7}=\left(1 / f_{6}+1 / f_{7}\right)^{-1}=214 \mathrm{~mm}$ is advantageous for two reasons. First, the flat surface of the plano-convex lens easily seals the drop-impact chamber against the ambient atmosphere (see Fig. 3.4). Second, the combination reduces spherical aberrations and improves the image quality on the CCD. To protect the delicate CCD from the high-energy beam, an attenuation by more than six orders of magnitude is required. The amount of attenuation must be insensitive to the polarization state of the light to capture all phase components at the same relative intensity. Otherwise, the sensor may only capture the beam profile for light with in-plane polarization which may be different from the profile for the out-of-plane component (a corresponding polarization state occurs for the beam-shaping in §3.4). As first attenuation step, we choose a wedged window at near-zero incidence. The final step is a neutral density (ND) filter that can be exchanged easily to match the sensitivity of the CCD to a change of laser energy. The position of lens $\mathrm{f}_{6,7}$ on the optical path relative to the drop as well as the position of the CCD thereafter, are fixed by the Gaussian lens formula [65], which is explained in more detail in §3.4.

For experiments that require laser energies much lower than $E_{\mathrm{L}, \min }$, we introduce an additional step of attenuation not shown in Fig. 3.2. Two wedged windows can be placed between the beam sampler and lens $\mathrm{f}_{3}$ in a Z-configuration to use the reflection of the first face of each window. The coating on the windows can be chosen to reach an additional attenuation by a factor of $10^{4}$ for two coated windows or a factor of $10^{2}$ in case one window is replaced by a laser-line mirror.

The free beam propagation described here is simple since it does not pose many restriction on the optical path. The distance between lens $f_{3}$ and $f_{4}$ is fixed to the sum of the focal length, $\mathrm{f}_{3}+\mathrm{f}_{4}$, to keep a collimated beam after the expansion. The position of lens $f_{5}$ relative to the drop is crucial to set the focusing condition. This leaves enough freedom to integrate other components in the beam path such as a $\lambda / 4$ - or $\lambda / 2$-plate to change the polarization state. In case more control over the beam shape is required we present an imaging and beam shaping technique in the following section. 


\subsection{Beam shaping and near-field imaging}

A drawback of a free beam propagation as described in the previous section is the deterioration of the flat-top beam profile as it propagates from the near-field to the far-field at the drop-impact location $(z=0 \mathrm{~mm})$. This can be solved by imaging the flat-top profile from the near-field (at a distance of about $400 \mathrm{~mm}$ from the laser head) to the drop-impact location (a distance of about $2500 \mathrm{~mm}$ ) as described in this section. In addition, the intrinsic properties of an imaging technique are beneficial when combined to a beam-shaping operation, which we explain in this section as well.

A near-field imaging is advantageous when a beam-shaping operation is performed at, or close to, the image plane that has its conjugated focal plane at the drop-impact location: the image is then propagated to its conjugated focal plane independent of the actual light path (of course, as long as clipping or spherical aberrations are minimized). As a consequence, a deviation in the propagation angle, inevitably introduced by the beam shaping, does not compromise the beam profile and, more importantly, its final position in the conjugated focal plane where the target of the laser impact is placed. As a result, once the imaging is implemented and properly aligned the beam shaping can be performed with minimum alignment effort. These considerations motivated the beam path presented here: a beam-shaping realized within the near-field distance such that any linear combination of the laser beam with a rotated version of itself can be imaged onto the drop. In addition, we place the CCD of the beam profiler in another conjugated focal plane behind the drop-impact location for the visualization of the beam shape as experienced by the drop (already explained briefly in § 3.3). Such a setup allows to study the response of the drop to different laser-beam profiles.

The complete laser-beam path including the beam shaping is shown in Fig. 3.3. As before, we make use of the polarization state of the main laser and create two orthogonal fields by a $\lambda / 2$-plate and a PBS. Two dove prisms allow for an arbitrary image rotation $\theta$ of the two fields. The multiple reflections inside the dove prism cause a slight change of the polarization state of each beam, which leads to a loss of laser energy of about $10 \%$ when the two fields are recombined in another PBS. Such an arrangement allows to create any linear combination of the input beam profile with a rotated version of itself. We use this feature to transform the elliptical beam profile to a near-circular profile to achieve axisymmetric impact conditions on the drop. The complete arrangement including attenuation as described in $\S 3.3$ is achieved within the near-field of the laser system (about $400 \mathrm{~mm}$ ).

In principle, the beam can be left freely propagating from this position (marked by the position of lens $f_{3}$ in Fig. 3.3) to the drop-impact location as 
described in §3.3. But as discussed before, any deviation in angle inevitably introduced by the dove prisms would lead to a large offset at the drop-impact location due to the long optical path of about $2500 \mathrm{~mm}$. This would need a careful re-alignment for each rotation of the beam, which we avoid by the near-field imaging. However, a beam-imaging technique imposes several restrictions upon our setup:

1. Any intermediate focus point of the high-energy laser beam needs to be covered with a vacuum tube to avoid optical breakdown in ambient air before the laser pulse hits the drop, i.e. for $z<0 \mathrm{~mm}$. Otherwise, the plasma at the focus spot absorbs and scatters nearly $100 \%$ of the incident laser energy.

2. No optical element can be placed in the vicinity of the drop to avoid the impact of fragments onto optical elements causing a damage or alteration to the laser-beam path in subsequent experiments.

3. The damage threshold $F_{\text {LIDT }}$ for each optical element must be respected as explained before in $\S 3.3$.

4. Two dichroic mirrors with a diameter of $50 \mathrm{~mm}$ at $45^{\circ}$ incidence need to be incorporated to allow sufficient access for the back-view visualization.

5. The optical elements placed at the laser entrance and exit of the dropimpact chamber need to allow for a proper seal of the inert atmosphere (compare Fig. 3.4).

6. In order to visualize the high-energy beam on the beam profiler we must attenuate the light once transmitted through the chamber. When the beam shaping is to be used, the laser beam contains an in-plane and out-of-plane polarization state and the attenuation must be performed equally for all phase components. We described a possible technique, a reflection at $0^{\circ}$ incidence, already in $\S 3.3$, but its implications on the path length must be considered here as well.

We design the laser path by an iterative procedure using geometrical optics, which is justified as the length scales to be considered here are large compared to the wavelength of the laser, that is $R_{0} / \lambda_{\mathrm{L}} \gg 1$. Each imaging step is then described by the Gaussian lens formula [65],

$$
\begin{aligned}
\frac{1}{s_{i}}+\frac{1}{s_{i}^{\prime}} & =\frac{1}{\mathrm{f}_{i}}, \\
M_{i} & =\frac{s_{i}^{\prime}}{s_{i}}=\frac{d_{i}^{\prime}}{d_{i}},
\end{aligned}
$$


that relates the distances from the object to the lens $s_{i}$ and the distance from the lens to the image $s_{i}^{\prime}$ to the focal length $\mathrm{f}_{i}$ and magnification $M_{i}$ of the imaging step $i$. The above equations need to satisfy the following restrictions: the magnification of the near-field from the first imaging step to the drop $M_{\text {drop }}=\prod_{1}^{\text {drop }} M_{i} \geq 2 R_{0} / d_{\mathrm{L}}=0.25$, in order to illuminate the drop completely. Likewise, the magnification from the drop to the CCD of the beam profiler $M_{\mathrm{BP}}=\prod_{\mathrm{drop}}^{\mathrm{BP}} M_{i} \leq d_{\mathrm{BP}} /\left(d_{\mathrm{L}} M_{\mathrm{drop}}\right) \approx 2.9$ to visualize the complete beam on the CCD.

As the required magnification to match the beam size in the near-field to the drop size satisfies $M_{\text {drop }}<1$, the occurrence of an intermediate focus point would inevitable be close to the conjugated focal plane if a collimated laser beam was imaged (the position of the intermediate focus point would be at the focal point of the imaging lens at $z<0 \mathrm{~mm}$ ). We then recognize that at least one additional imaging step is required to create a diverging beam such that the intermediate focus point is shifted to a position $z>0 \mathrm{~mm}$ while the conjugated focal plane is kept at $z=0 \mathrm{~mm}$. Despite those considerations and restrictions, the described system is still underdetermined given the choices of optical elements available. Therefore, we first pick a set of optical elements, choose $s_{1}$ and calculate any remaining quantities according to Eqs. (3.1) and (3.2). We calculate the local fluence at all optical elements to evaluate our choice based on restrictions 1 to 6 . Next, we need to determine and evaluate the consequences for the back-view visualization.

The illumination for the back-view visualization needs to be partially incorporated in the optical path of the main laser given the position of the dichroic mirrors. We calculate the image of the light source along its optical path based on Eqs. (3.1) and (3.2). However, the optical elements that are part of the combined optical path of the main laser and back-view visualization are already fixed in terms of their position and focal length. In addition, the divergent nature of the light source requires the first lens in the back-view path to be a lens (or focusing mirror) with a large numerical aperture (NA) to capture most light of the source (we select an aspheric collimator lens $\mathrm{f}_{8}=32 \mathrm{~mm}$ ). This narrows down the choice for the set of lenses already considerably. The remaining criterion to check for the back-view illumination is then given by its sole purpose: the required magnification to illuminate the drop-impact position in a uniform way. As will be discussed in $\S 3.5$ the size of the field of view (FOV) $d_{\mathrm{FOV}}=20 R_{0}=20 \mathrm{~mm}$ and given the lateral extension of the light source, typically about $d_{\text {Light }}=8 \mathrm{~mm}$, we find for the required magnification $M_{\mathrm{BV}}=d_{\mathrm{FOV}} / d_{\text {Light }} \geq 2.5$.

Our solution to above problem is illustrated in Fig. 3.3 with one imaging step for the laser beam path before and after the drop. The back-view 
illumination includes two imaging steps before the drop, which is necessary to compensate for the effect of the shared lens $\mathrm{f}_{6,7}=214 \mathrm{~mm}$ in the backview by lens $f_{9}=50 \mathrm{~mm}$. The image of the laser beam at the position of lens $\mathrm{f}_{3}=300 \mathrm{~mm}$ is propagated to the drop position (image and conjugated focal planes are highlighted in red in Fig. 3.3). These arrangements leads to the first intermediate focus after lens $\mathrm{f}_{3}$, which is covered by a vacuum tube. As required, the next conjugated focal plane created by $f_{5}=200 \mathrm{~mm}$ at the drop-impact position is located before the second intermediate focus at about $z=30 \mathrm{~mm}$ (see also Fig. 3.4). This means that no laser energy is lost in an intermediate focus by plasma generation before the impact onto the drop.

The focus point behind the drop, i.e. the point of optical breakdown and plasma generation, cannot be covered by a vacuum tube. However, the distance of about $30 \mathrm{~mm}$ between the drop and the focus point is sufficiently large in order not to interfere with our side- and back-view visualization. Care must be taken to measure the beam profile, which is obtained in the absence of the drop by the beam profiler (Fig. 3.3), at energies low enough to avoid optical breakdown. Please note, that the intermediate focus just before energy meter 1 does not require a vacuum enclosure as the energy in that part of the laser-beam path is far below the threshold for optical breakdown. Therefore, lens $f_{3}$ is not only used to image the near-field but also used to match the laser beam to the size of the sensor for energy meter 1 .

Figure 3.3 also illustrates the point that limits the maximum size of the FOV in the back-view: at the position of lens $\mathrm{f}_{6,7}$ the path of the back-view exhibits its maximum lateral extension. Reversing the order of lens $f_{6,7}$ and the dichroic mirror could solve this issue but requires an additional window to seal the chamber before the dichroic mirror can be placed. This would make the dichroic mirror the element that clips the FOV leading to no further improvement. Using lens $\mathrm{f}_{6,7}$ to seal the chamber as sketched in Fig. 3.4 minimizes the required path length and is thus our preferred choice. In this configuration, the light enters the back-view at the bottom of Fig. 3.3 and forms

Figure 3.3: (Figure is found on the following page.) Unfolded optical path for the main laser and back-view visualization. The drawings for the beam imaging and back-view are to scale and aligned, but make use of two different scales in $\vec{e}_{z}$ - and $\vec{e}_{y}$-direction to accommodate the complete beam path of about $2500 \mathrm{~mm}$ in length, while the maximum lateral extension in $\vec{e}_{y}$ is only $30 \mathrm{~mm}$. As a consequence, the split-off at energy meter 1 and the beam profiler are shown at $45^{\circ} \mathrm{AOI}$ for illustration, whereas it is actually performed at near-zero AOI. Similarly, the reflections at $45^{\circ} \mathrm{AOI}$ at any mirror are omitted in this unfolded representation of the optical path (mirror M1 is marked as reference point to compare with Fig. 3.2). 


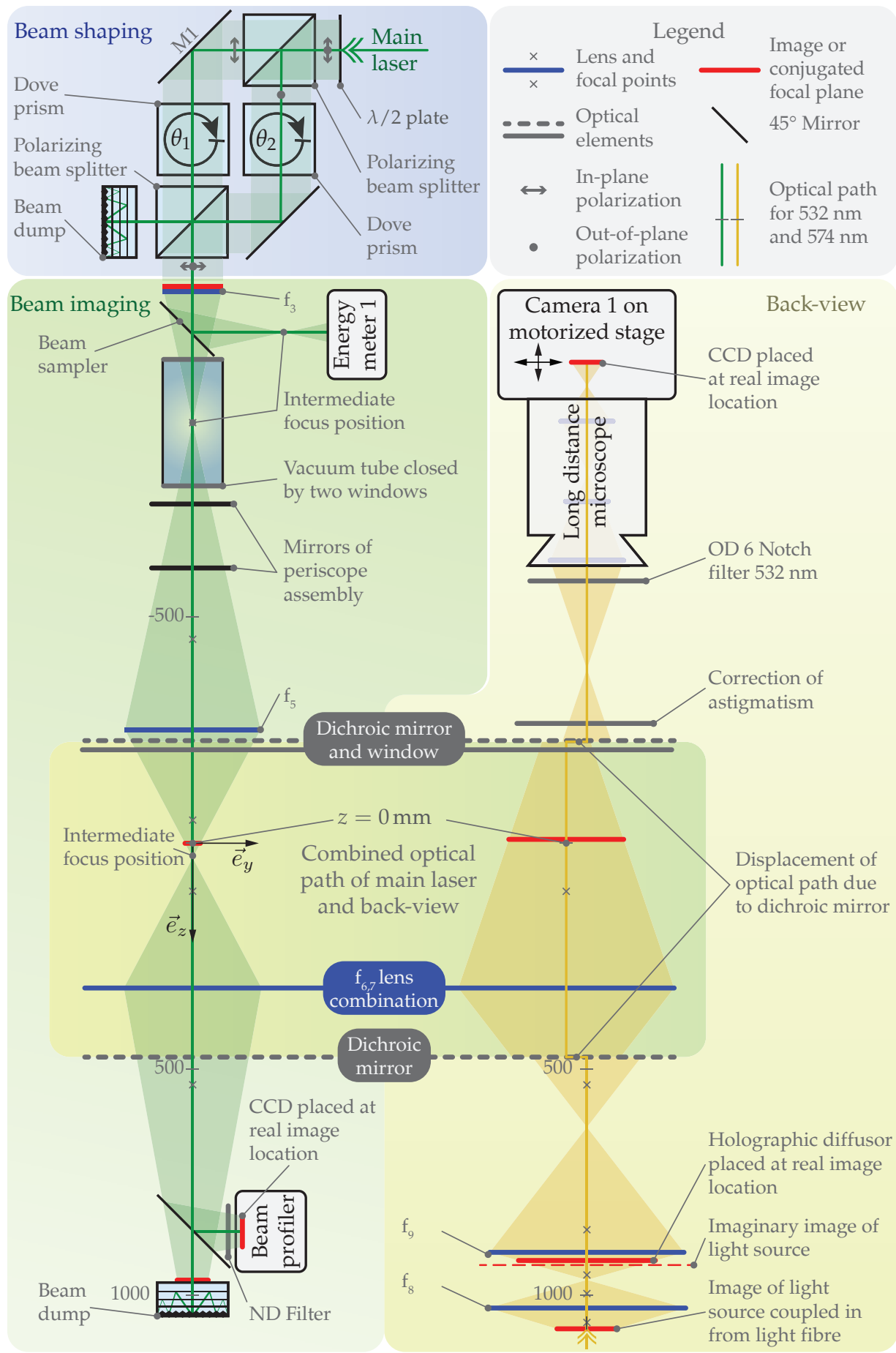

Figure 3.3: (Continued from the previous page.) 
a real image after lens $f_{8}$. This image is propagated by lens $f_{9}$ and $f_{6,7}$ to the drop impact location with the appropriate magnification. The light source is divergent as illustrated in Fig. 3.3 and light is lost in the additional imaging step. Therefore, a diffusor with fine control over diffusing angles is required (holographic diffusers by Edmund Optics, version with $5^{\circ}$ to $20^{\circ}$ diffusing angle). This selection allows to find a compromise between light intensity and uniformity. Sufficient light must be captured by each camera as described in $\S 3.5$ while the FOV needs to be illuminated uniformly to ease subsequent image analysis and interpretation.

\subsection{Visualization}

The purpose of our setup is to study the fluid-dynamic response of the liquid target to a laser impact. To this end, the experimental apparatus allows for extensive visualization of the complete process by high-speed and stroboscopic imaging as explained in this section. The response of the liquid drop to the laser impact involves several timescales. The laser impact itself takes place on a nanosecond timescale set by $\tau_{p}=5 \mathrm{~ns}$. The timescale of the drop dynamics ranges from a few microseconds (compare Fig. 3.1) up to the capillary timescale $\tau_{c}=\left(\rho R_{0}^{3} / \gamma\right)^{1 / 2} \approx 1 \mathrm{~ms}$, where $\rho$ and $\gamma$ are the liquid density and surface tension, respectively. The frame rate $f_{\text {cam }}$ to resolve the largest timescale $\tau_{c}$ properly is at least 5000 frames per second (fps) following typical criteria for high-speed imaging [66]. Any other process in our experiment requires a frame rate of orders of magnitude larger.

The spatial scale of interest is set by $R_{0}$ but needs to allow for an expansion of the initially spherical drop into a larger geometry, where preliminary experiment suggested a field of view (FOV) of about $d_{\mathrm{FOV}}=20 R_{0}=20 \mathrm{~mm}$ in diameter. However, the smallest features to be resolved are those of drop fragments $\ll R_{0}$, see Fig. 3.1. This requires an imaging technique with a high spatial resolution to capture both, the large FOV and the details of drop fragmentation. Furthermore, the liquid target is allowed to move freely in space after the laser impact, which requires that no optical element is present in the vicinity of the drop to avoid liquid fragments reaching the optics and causing a damage or alteration to the laser-beam path in subsequent experiments. Since the laser impact can be violent as shown in Fig. 3.1, a distance from the drop of about $100 R_{0}$ to $300 R_{0}$ for each optical element is preferred. Thus, imaging at a large working distance in comparison to $d_{\mathrm{FOV}}$ is required.

Our visualization techniques must allow for an optimum temporal and spatial resolution given the aforementioned considerations, which is difficult to meet with a single system. Therefore, we choose for high-speed and strobo- 
scopic imaging [66] techniques to be used interchangeable in our setup. The latter requires an experiment with a high degree of reproducibility to allow for multiple experiments being recorded under identical conditions but imaged at different times to give the perception of a continuous movie.

To image the laser impact and drop onto the different cameras we use a long-distance microscope (LDM, K2 DistaMax by Infinity Photo-Optical Company) for both high-speed and stroboscopic imaging. It supports fullframe camera chip sizes ( $36 \mathrm{~mm} \times 24 \mathrm{~mm}$ ) as found in the high-resolution cameras $\left(4008 \times 2672 \sim 10^{7}\right.$ pixels) that we use for stroboscopic imaging. The high-speed cameras feature smaller chip sizes $(20 \mathrm{~mm} \times 20 \mathrm{~mm}, 1024 \times 1024 \sim$ $10^{6}$ pixels) that require the magnification to be adapted by exchangeable objectives. Fortunately, the magnification required in our case, given the FOV and camera chip sizes, is of the order of one, which does not pose great demands for the imaging optics except the large working distance.

The high-speed system consists of a continuous light source (LS-M352A metal halide light source by SUMITA Optical Glass for the back-view and a MAX-303 xenon light source by Asahi Spectra for the side-view) that is prepared by critical illumination (see Fig. 3.2). An aspheric collimator lens (ACL5040U-A by Thorlabs) creates an image of the light source on a diffusor with a high transmission efficiency (ED1-C50 by Thorlabs and holographic diffusers by Edmund Optics). A second aspheric lens (AL50100-A) projects a magnified version of that image to fill the FOV of the side-view in a uniform way (the optical path for the back-view is described in §3.4). To allow for a precise alignment the optics for each light source are mounted on a yawand-pitch platform on top of a translational stage. The high-speed cameras (FASTCAM SA-X2 and FASTCAM SA1.1 by Photron for the back- and sideview, respectively) are mounted on translational stages. The one for the back-view is motorized to move the image plane along the optical axis $\vec{e}_{z}$ by software control. This helps to tune the camera position to visualize the part of the drop dynamics that is of interest: the drop is propelled along $\vec{e}_{z}$ and, therefore, leaves the depth of field at some point in case the camera position is fixed, even at nearly-closed aperture of the LDM.

The back-view poses another challenge: it needs to be combined with the laser-beam path as already discussed in $\S 3.4$. This is accomplished by two custom-made dichroic mirrors (Laseroptik, Garbsen). These long-pass filter are phase preserving to keep the polarization state, have a high $F_{\text {LIDT }}$ and a near- $100 \%$ transmission for $\lambda \geq 574 \mathrm{~nm}$. The latter is a requirement for the monochromatic light source that we use for stroboscopic imaging.

The light path of the back-view and its alignment in reverse direction with the laser-beam path is shown to scale in Fig. 3.3. The direction of propagation is reversed to avoid forward scattering or even direct laser radiation into 
the high-speed camera in case any protective measure such as a notch filter fails. The thickness of the glass substrate of the dichroic mirrors introduces a considerable displacement of the optical path (Fig. 3.3), which leads to an astigmatism in the back-view that has to be corrected for proper imaging. A substrate of the same material and dimensions but with an anti-reflective coating is introduced just behind the dichroic mirror rotated by $90^{\circ}$ about $\vec{e}_{z}$. This element introduces a displacement in $\vec{e}_{x}$-direction (not visible in Fig. 3.3) to compensate the astigmatism by lengthening the optical path in the direction orthogonal to the displacement introduced by the dichroic mirror. Despite the additional optics and with careful alignment the amount of light captured by the high-speed cameras is enough to use even the shortest possible exposure time of $293 \mathrm{~ns}$ for the FASTCAM SA-X2. This option is very advantageous for our problem that involves multiple timescales. It allows us to image early drop fragments without motion blur despite their small size and high speed.

The purpose of the stroboscopic visualization is to achieve far higher temporal and optical resolution as with high-speed imaging. We use pulsed light sources with exposure times of a few nanoseconds to illuminate the scene of interest and capture the image on high-resolution cameras. Both cameras (pco.4000 by PCO AG and Bigeye G-1100B Cool by Allied Vision Technologies) feature the same chip (Truesense KAI-11002 with $4008 \times 2672 \sim 10^{7}$ pixels) and mounting options (F-mount by Nikon), which allows identical LDM configurations to be used in side-view and in back-view. The pco.4000 offers a double-image mode that allows to capture two successive frames of the same experiment with an inter-frame time of less than $1 \mu \mathrm{s}$. This feature can be used to quantify velocity maps, e.g. particle tracking and particel imaging velocimetry [66]. Of course, it also requires a pulsed light source capable of delivering two pulses of light in rapid succession.

We use a dual-cavity, Q-switched, and frequency-doubled Nd:YAG laser system (EverGreen 200 by Quantel) as light source for stroboscopic imaging that is capable of delivering two pulses of laser light with a duration of $10 \mathrm{~ns}$ and an arbitrary delay between the two pulses (see Fig. 3.2). The green laser is first attenuated based on polarization in the same way as the main laser described in §3.3. Next, the beam is split in two parts by another $\lambda / 2$-plate and a PBS. The coherence of the laser light, which would lead to interference and speckles when used directly for visualization, is removed by incoherent laser-induced fluorescence light illumination (iLIF) [67]. In this technique, the laser beam is expanded into a dye solution or solid plate where the incoming light at a wavelength of $532 \mathrm{~nm}$ is absorbed to a resonantly excited state. The subsequent fluorescence emission at a wavelength of 574 to $580 \mathrm{~nm}$ preserves the 10-ns pulse duration of the incoming laser pulse (High Efficiency Diffuser by LaVision). The incoherent, monochromatic light is coupled into two optical 
fibers to allow an arbitrary placement of the light sources in the setup.

All visualization systems described so far record grayscale images chosen for its superior light sensitivity [66]. A system to record color information requires light of a broad spectrum for illumination, which is challenging for the back-view as the dichroic mirrors act as a long-pass filter. However, the side-view only requires a notch filter minimizing the effect on the spectrum and offers a possibility to record color images. We use a consumer digital SLR camera (Nikon D5100) to record color images stroboscopically and a pulsed light source (NANOLITE KL-K by HSPS) for flash illumination. The light source delivers a high-intensity light pulse of $8 \mathrm{~ns}$ in duration. It makes use of an electric discharge to create a plasma spark, which we image in a similar way as we described for the monochromatic light sources.

The images taken with the camera systems and beam profiler are calibrated by taking images of semi-transparent calibration targets placed at the dropimpact location. We use high-precision Ronchi rulings by Edmund Optics that cover the complete FOV or fixed-frequency grid distortion targets by Thorlabs. This allows to translate image dimensions in each view to world units in the lab reference frame. The spatial relation among the different views can be inferred either from a unique point on the calibration targets captured in all views at the same time or from the spherical drop itself. The initial drop before laser impact is recorded in all views, sets the origin for our coordinate system, and is therefore a natural reference point for calibration.

\subsection{Drop impact chamber}

The chamber encloses the laser impact in an inert atmosphere and limits the area where any fragments, vapor, or aerosol of the liquid may go after the laser impact. The chamber is built from a standard $60-\mathrm{mm}$ cage system by Thorlabs with custom alterations to seal the aluminum housing and connect flow in- and outlet ports, see Fig. 3.4. The dimensions of the chamber are such that common $50 \mathrm{~mm}$ or 2 inch optics can be mounted directly. The distances between the drop and the first optical element along the $\vec{e}_{y}$ - and $\vec{e}_{z}$-axis are maximized under the restriction imposed by the near-field imaging and visualization. This leads to the cross-shaped chamber design as seen in the top view in Fig. 3.2.

The drop generator is found on top of the chamber in Fig. 3.4 and fed by a constant flow rate $\dot{V}$ driven by a syringe pump (PHD Ultra by Harvard Apparatus, not shown). The design repetition rate $f_{\text {exp }}$ requires $\dot{V}=4 / 3 \pi R_{0}^{3} f_{\exp }=$ $0.25 \mathrm{ml} / \mathrm{min}$ for a drop of initial radius $R_{0}=1 \mathrm{~mm}$. A suitable principle for drop generation in this case is the quasi-static pinch-off at the tip of a capillary 


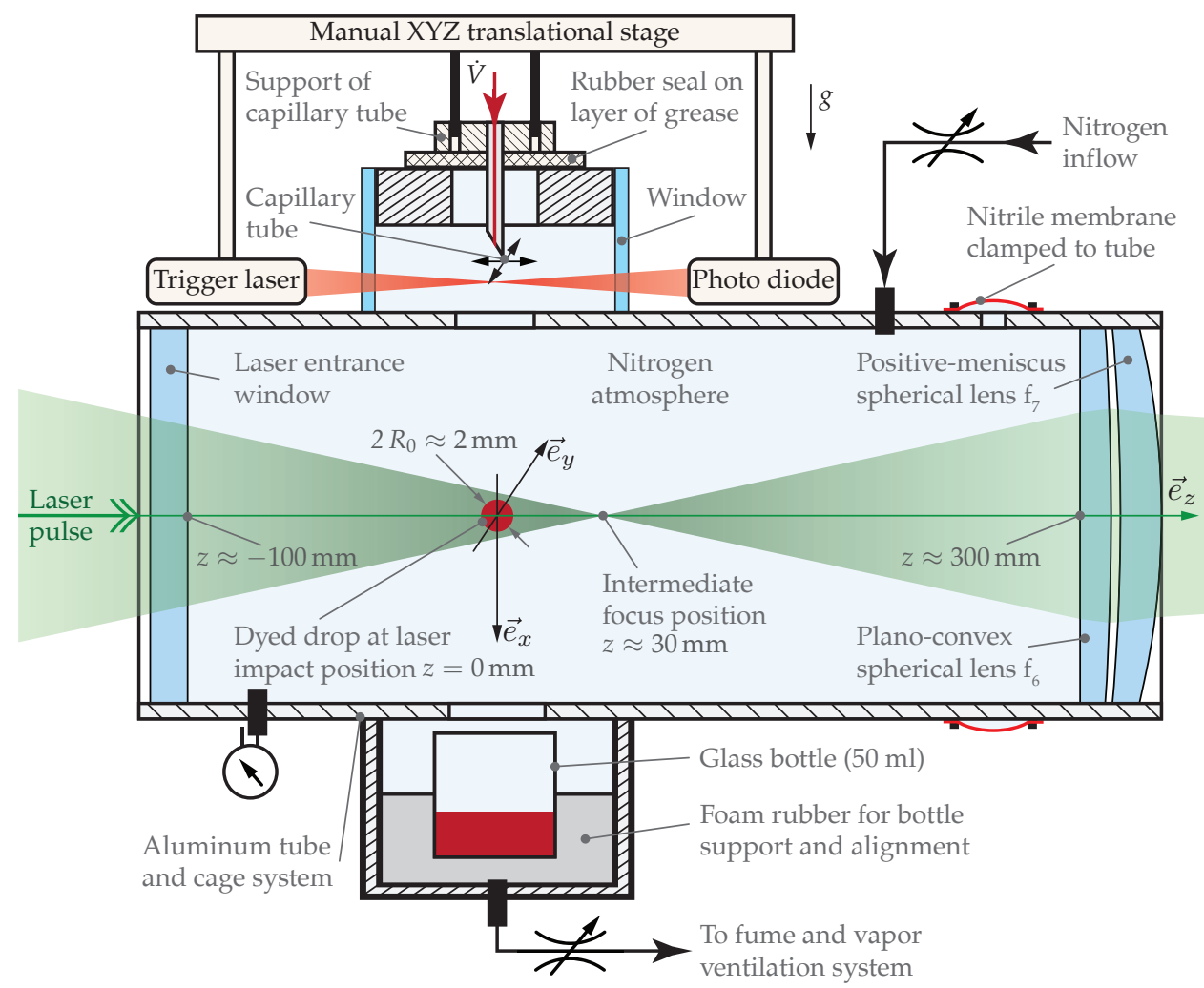

Figure 3.4: A sketch of the drop-impact chamber in a cross-section illustrating the moment of laser impact (dimensions not to scale, intermediate focus position as calculated in §3.4). The inner dimensions of the aluminum chamber are such that common 50-mm and 2-inch optics can be mounted directly. The chamber is sealed against ambient air and multiple nitrogen inlet ports are placed close to each optical element (illustrated for the lens combination at the right). A single outlet at the bottom of the chamber is connected to a low-pressure ventilation system leading to gentle flow away from the optical elements to the bottom of the chamber. Any excessive liquid that does not evaporate over time is collected at the bottom of the chamber in a glass bottle that allows for a quick exchange.

tube. The tube's outer radius $R_{c, o}$ needs to be smaller than the drop for a reproducible position of the drop detachment under the influence of gravity $g$. The Weber number $\mathrm{We}_{c}$ of the capillary tube relates the kinetic energy of the liquid to its surface energy and can be expressed as $\mathrm{We}_{c}=\rho R_{0} \dot{V}^{2} /\left(\pi^{2} \gamma R_{c, i}^{4}\right)$, where the kinetic energy is determined by the flow speed inside the capillary with inner radius $R_{c, i}$. Even for a regular 30-gauge needle with $R_{c, o} / R_{0}=1 / 3$ is $\mathrm{We}_{c}=0.6<1$, which means that control over the pinch-off position can be ensured for such a small needle while the drop generation is purely controlled 
by surface-tension forces and gravity. Any dripping, or even jetting [50] at $\mathrm{We}_{c} \gg 1$, is only observed in the case that $R_{c}$ is further decreased, for example in the event of clogging inside the capillary tube.

A drawback of the proposed method is the lack to vary the drop radius $R_{0}$ over a large range. $R_{0}$ is set by the balance of surface tension and gravity and scales like $R_{0} \sim \mathcal{K}^{-2 / 3} R_{c, 0}^{1 / 3}$, where $\mathcal{K}^{-1}=(\gamma /(\rho g))^{1 / 2}$ is the capillary length [68]. The weak dependence of $R_{0}$ on $R_{c, o}$ does not allow for a large variation, but the drop generation mechanism is very robust and leads to a stable $R_{0} \approx 1 \mathrm{~mm}$ for the liquids described in $\S 3.7$.

Once the drop detaches at the tip of the capillary tube, it falls down towards the laser-impact position under the influence of gravity while it relaxes to a spherical shape. The drop masks a photodiode (PDA36A by Thorlabs) that is illuminated by continuous-wave laser diode (CPS635R by Thorlabs) emitting light at $635 \mathrm{~nm}$. The low-power laser diode is focused at the position where the drop passes as illustrated in Fig. 3.4 to create a precise trigger on the passage of the drop. The trigger finally leads to the main laser emitting a pulse of light that enters from the left through a window, hits the drop at $z=0 \mathrm{~m}$, and exits to the right through the lens combination $\mathrm{f}_{6,7}$. The complete arrangement of the trigger laser, photodiode, and capillary can be moved relative to the drop chamber allowing for a free positioning of the drop along $\vec{e}_{y}$ and $\vec{e}_{z}$ without the need to change the trigger alignment. The details on the timing and control, used to align the drop relative to the laser beam along $\vec{e}_{x}$, are left for $\S 3.8$.

An elastic membrane visible from the outside of the chamber as shown in Fig. 3.4 serves two purposes. First, it is a visual indication of a slight over-pressure inside the chamber relative to ambient conditions. The overpressure is controlled by two flow-vales and a static pressure regulator (not shown) and ensures that no oxygen enters the chamber to prevent an explosive mixture with the vapor of a flammable liquid. Second, the membrane serves as safety release valve when an unexpected pressure build-up occurs during an explosion of flammable liquids that could not be prevented by other means. As discussed, the intermediate focus position at $z>0 \mathrm{~mm}$ cannot be covered by a vacuum tube and is a likely spot for the ignition of an explosion as previous experiments have confirmed. The over-pressure can also be checked with a digital differential pressure gauge, that offers an audible feedback in case the over-pressure falls below a certain limit.

\subsection{Liquids}

We aim to control the deposition of laser energy in the liquid by adding a dye such that the linear absorption coefficient $\alpha$ at the wavelength $\lambda_{\mathrm{L}}$ can be 
varied in comparison to $R_{0}$. Water is a very good model system in terms of its well-known physical properties, safe operation, availability and previous research on laser-matter interaction [20]. However, the linear absorption coefficient [69] $\alpha=0.0447 \mathrm{~m}^{-1}$ of pure water at $\lambda_{\mathrm{L}}=532 \mathrm{~nm}$ is negligible small in comparison to the scale of our system, i.e. $R_{0} \alpha \ll 1$. The opposite regime, $R_{0} \alpha \gg 1$, is of great interest, since the laser energy is then limited to a superficial layer of the drop, the thickness of which scales as $\delta \sim 1 / \alpha$, which leads to a high energy density upon laser impact. This provokes a more violent fluid-dynamic response as the kinetic energy imparted by the drop is increased as explained in $\S 4$. To overcome the limitation and actually control the penetration depth of laser light $\delta$ at $\lambda_{\mathrm{L}}=532 \mathrm{~nm}$ into the liquid phase, we solve a dye at variable concentration in otherwise pure water.

To explore the range $R_{0} \alpha \gg 1$ a dye with a high solubility is required. We use the dye Acid-Red-1 (chemical abstracts service registry number (CASRN): 3734-67-6, product number 210633 by Sigma-Aldrich), which is also used in coatings or inks and as such is available at large quantities and at reasonable cost. We filter each dye-water solution during preparation and place a syringe filter with a pore size of $200 \mathrm{~nm}$ in front of the capillary tube as final stage of filtration. This is of importance when performing experiments with a solution close to the solubility limit to ensure that no particles or dye agglomerates change the dynamic response of the system. This potential effect of particles in the liquid on the fluid dynamics is also why we avoid to use pigment dyes. Instead, we may use black (IJC-5900) and magenta (IJC-5920) pigment-free inks by Sensient Imaging Technologies.

We measured the absorption coefficient for the aqueous Acid-Red-1 solution over a large range of mass fractions of dye $w$ and find $\alpha=\alpha_{0} w$ with $\alpha_{0}=(8.05 \pm 0.2) \times 10^{6} \mathrm{~m}^{-1}$, compare Fig. 3.5. Our results are based on measuring the transmitted energy through liquid samples of known optical path length and are in very good agreement with measurements performed at the solubility limit with a Z-scan technique [70]. To conclude, the aqueous Acid-Red-1 system allows to vary the laser-matter interaction in the range $5 \times 10^{-5} \leq R_{0} \alpha \leq 400$, where the lower limit is given by pure water and the upper limit by the solubility limit $w_{s}=0.05$ as stated by the manufacturer.

A second solution consists of methyl ethyl ketone (MEK, CASRN: 78-933, product number 04380 by Sigma-Aldrich) as the solvent and Oil-Red-O (CASRN: 1320-06-5, product number 1320-06-5 by Sigma-Aldrich) as the dye. The solvent MEK exhibits a lower latent heat of vaporization as compared to water, which is expected to cause an even stronger drop response in terms of induced flow velocities for a given normalized penetration depth, i.e. $\left(R_{0} \alpha\right)^{-1}$, and laser energy as explained in $§ 4$. Initial experiments indeed show that 


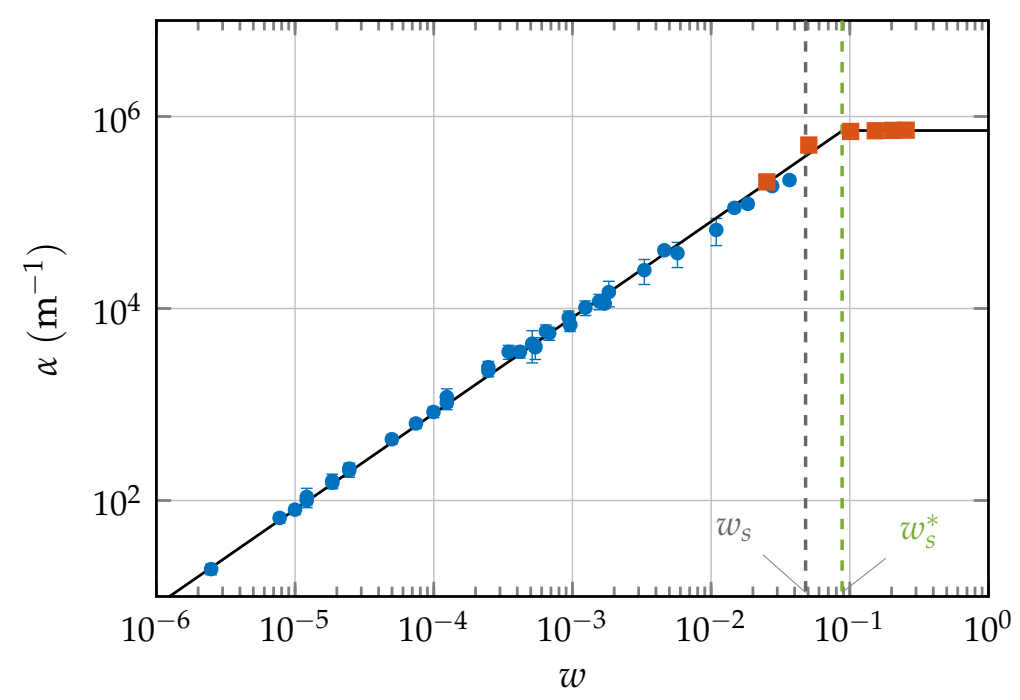

Figure 3.5: Linear absorption coefficient $\alpha$ of an aqueous Acid-Red- 1 solution as function of the mass fraction $w$ of dissolved dye. The transmission measurements (circle marker $\bullet$ ) confirm $\alpha=\alpha_{0} w$ with $\alpha_{0}=(8.05 \pm 0.2) \times 10^{6} \mathrm{~m}^{-1}$ to be valid up to the solubility limit $w_{s}=0.05$ as reported by the manufacturer, i.e. for $w \leq w_{s}$. At even higher concentration the absorption coefficients saturates for $w \geq w_{s}^{*}=0.09$, which was confirmed with a Z-scan technique (square marker $\square$ ) by Gayathri and Ramalingam [70]. The solid line (-) is the linear $\left(w \leq w_{s}^{*}\right)$ and constant $\left(w>w_{s}^{*}\right)$ fit, where our impact experiments are performed in the unsaturated regime.

this solution also operates at $R_{0} \alpha \gg 1$ and with a much stronger response as compared to any aqueous Acid-Red-1 solution.

A liquid property very important in our experiments is the surface tension $\gamma$ as the late-time fluid dynamics are determined by surface-tension forces in comparison to the kinetic energy that the drop gained upon laser impact. Even the smallest amounts of surfactants may affect the surface tension of a liquid sample. Since we cannot exclude surface-active impurities in our solutions, i.e. due to left-overs from the dye manufacturing process, we measure the surface tension for both liquid systems. Pendant-drop measurements [68] are performed in a contour-analysis system (OCA by DataPhysics Instruments) that allows to record the surface tension of the solution in the pendant drop as function of time, see Fig. 3.6. The results show that on timescales relevant to the laser impact on a drop, where the largest timescale is of the order of a few milliseconds, the surface tension of the solution can be approximated by the value of the pure solvent within $\pm 2 \%$, see Table 3.1 for the measured values. However, for experiments taking place on a longer timescale of several seconds or even minutes the surface tension changes and decreases by more than 


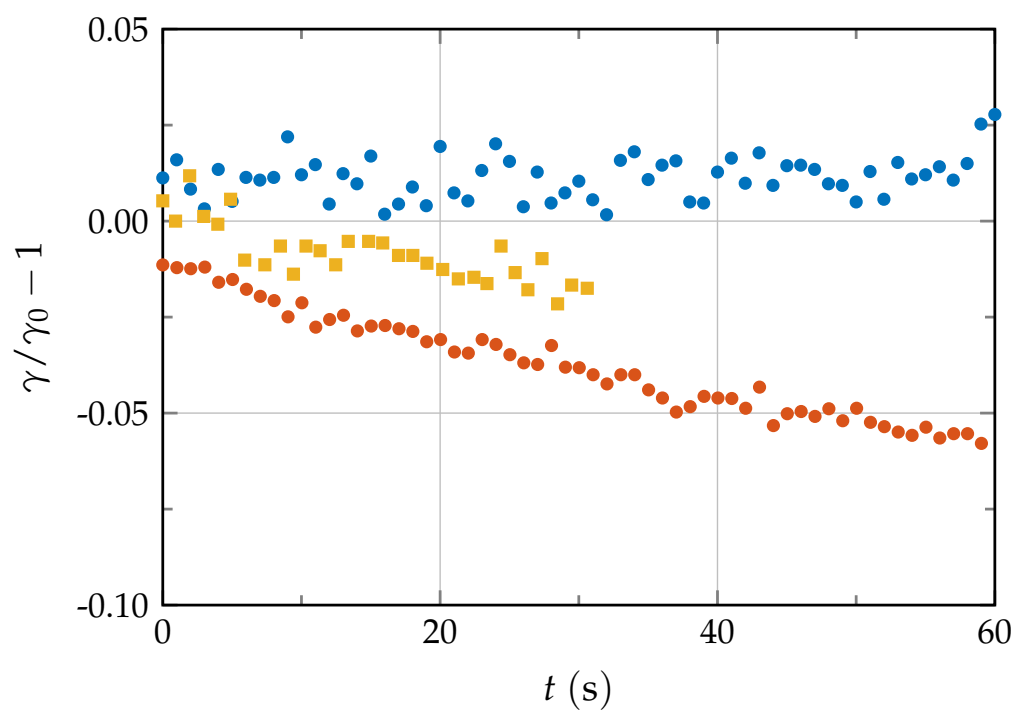

Figure 3.6: Surface tension as function of time $t$ during pendant drop measurements. The time $t \approx 0$ s corresponds the moment the drop is formed at the tip of a capillary tube. The surface tension of each solution $\gamma$ is compared to the surface tension of the pure solvent $\gamma_{0}$ for three different solutions: an aqueous Acid-Red-1 solution at low $\left(w=0.002 w_{s}\right.$, circle marker $\left.\bullet\right)$ and high $\left(w=0.75 w_{s}\right.$, circle marker $\bullet$ mass concentration of dye, as well as Oil-Red-O dissolved in MEK at approximately half the solubility limit $\left(w=0.5 w_{s} \text {, square marker }\right)^{-}$.

$30 \%$ for the aqueous Acid-Red- 1 solution after 20 min at high concentration (not shown in the figure). Such long measurement durations are excluded for the volatile MEK solution, which then explains why the measurement is stopped after $30 \mathrm{~s}$ in Fig. 3.6.

The solvent MEK requires the drop-impact chamber to have an inert atmosphere, since it is a hazardous substance that can be ignited under ambient temperature conditions [71]. A further complication is the rapid evaporation of MEK, which is partially due to its low latent heat of vaporization and which can affect the drop-generation control mechanism that was described in §3.6. The evaporation of the drop at the tip of the capillary leads to an increase of dye concentration and, once the solubility limit is reached locally, causes an agglomeration of dye particles, an effect well-known as a cause for coffee stains [72]. These particles form structures around the tip of the capillary tube leading to an unsteady pinch-off behavior of the drop or, even worse, to a complete clogging of the capillary once the flow rate is too low.

We avoid the clogging and stain formation by an active feedback loop controlling the volume flow rate $\dot{V}$ based on the drop pinch-off frequency $f_{\exp }$. 
The syringe pump, which sets $\dot{V}$, is not switched off between experiments but set to a low volume flow that ensures a drop pinch-off approximately every $20 \mathrm{~s}$. Before an actual experiment, the feedback loop is started to tune the flow rate to match the design repetition rate $f_{\exp }=1 \mathrm{~Hz}$ within $\pm 1 \%$. This ensures that the capillary and its tip are flushed and residual dye particles are removed.

Table 3.1: Liquid properties of an aqueous Acid-Red-1 solution and Oil-Red-O dissolved in methyl ethyl ketone (MEK). The surface tension $\gamma$ of the solution is unaffected by the addition of the dye on short timescale, i.e. $t<1 \mathrm{~s}$, and can be approximated by the value of the pure solvent. Likewise, the density is given by the value of the pure solvent, which is valid for a low dye mass fraction, i.e. $w \ll 1$.

\begin{tabular}{llcc}
\hline parameter & unit & $\begin{array}{c}\mathrm{H}_{2} \mathrm{O} \& \\
\text { Acid-Red-1 }\end{array}$ & $\begin{array}{c}\text { MEK \& } \\
\text { Oil-Red-O }\end{array}$ \\
\hline$\rho$ & liquid density $\left(\mathrm{kg} / \mathrm{m}^{3}\right)$ & 998 & 805 \\
$\gamma$ & surface tension $(\mathrm{N} / \mathrm{m})$ & 0.072 & 0.025 \\
$\alpha_{0}$ & absorption coefficient $(1 / \mathrm{m})$ & $(8.05 \pm 0.2) \times 10^{6}$ & - \\
\hline
\end{tabular}

\subsection{System control and timing}

The control system for the lasers, cameras, energy meters, and auxiliary devices such as the syringe pump or motorized stages needs to ensure a precise timing and deterministic control of the devices. However, the timescales that need to be respected by a certain device may differ as illustrated in Fig. 3.7 (a). The devices in the asynchronous group do not communicate directly with each other but only via the control software running on a computer (Z420 workstation by Hewlett Packard, Windows 7 by Microsoft as operating system, control software is implemented in MATLAB by MathWorks). The precision of the timing between devices in this group is set by the combined processing time of the control software and operating system. Since we do not use any real-time operating system we cannot guarantee deterministic synchronization between the devices and, therefore, settings are applied in an asynchronous fashion.

By contrast, the synchronous control group consists of hardware that needs to interact with each other deterministically on a timescale as small as a few nanoseconds. This is achieved by hard-wired triggers transmitted directly among devices by a transistor-transistor logic (TTL). The precise timing among signals and their logical relation is controlled by a programmable pulse-delay generator (BNC575 by Berkeley Nucleonics Corporation with 
extended firmware and a precision of $250 \mathrm{ps}$ ). The pulse-delay generator is programmed before an actual set of experiments in an asynchronous fashion from the control software but it operates independently during the actual experiments.

For example, during a stroboscopic measurement we may change the flow rate of the syringe pump but cannot know for sure when the setting is applied by the device due to residual flow in the long feed lines. In principle, this is not a problem, since the effect of the flow rate, in our experiment the repetition rate of the drop pinch-off, is measured by a frequency counter in a synchronized way. Similarly, when changing the position of a $\lambda / 2$-plate in a motorized mount we can monitor its effect by an energy meter. However, to ensure correct synchronization between devices of the different groups, the experiment needs to be stopped first. Then, the settings need to be applied, while the state of any asynchronous device is read out until the new settings are confirmed. Only then, the experiment can be restarted for a known and synchronized setting.

The control and timing of the devices is described along Fig. 3.7 for a stroboscopic experiment as it poses the highest demands in terms of reproducibility and a precise synchronization:

1. The function generator (33600A by Keysight) is synchronized to its internal clock feeding pulses at a fixed frequency of $1 \mathrm{~Hz}$ to the main laser and pulse-delay generator, both connected to channel A (please note that Fig. 3.7 shows the case where the function generator is already synchronized to the drop generation). The actual output of the pulsedelay generator is off to prohibit any actual laser output or devices being activated. However, from this point onward the cavity of the main laser is pumped by its flash-lamp allowing for the temperature-stabilized cavity to reach thermal equilibrium.

2. The syringe pump is switched on, drop generation starts and each drop generates a trigger signal at the photodiode that is received by the function generator. The signal is feed back to a frequency counter (J-50MBYAG-USB by Coherent, an energy meter used for testing or calibration but also capable of frequency counting) connected to channel B of the function generator.

3. At this point the drop generation does not yet affect any other device (main laser and pulse-delay generator are still synchronized to a fixed frequency), allowing to optimize the syringe pump. An active feedback loop controls the flow rate of the pump until the design repetition rate of the experiment $f_{\exp }=1 \mathrm{~Hz}$ is reached within $\pm 1 \%$, see $\S 3.7$. 
(a)

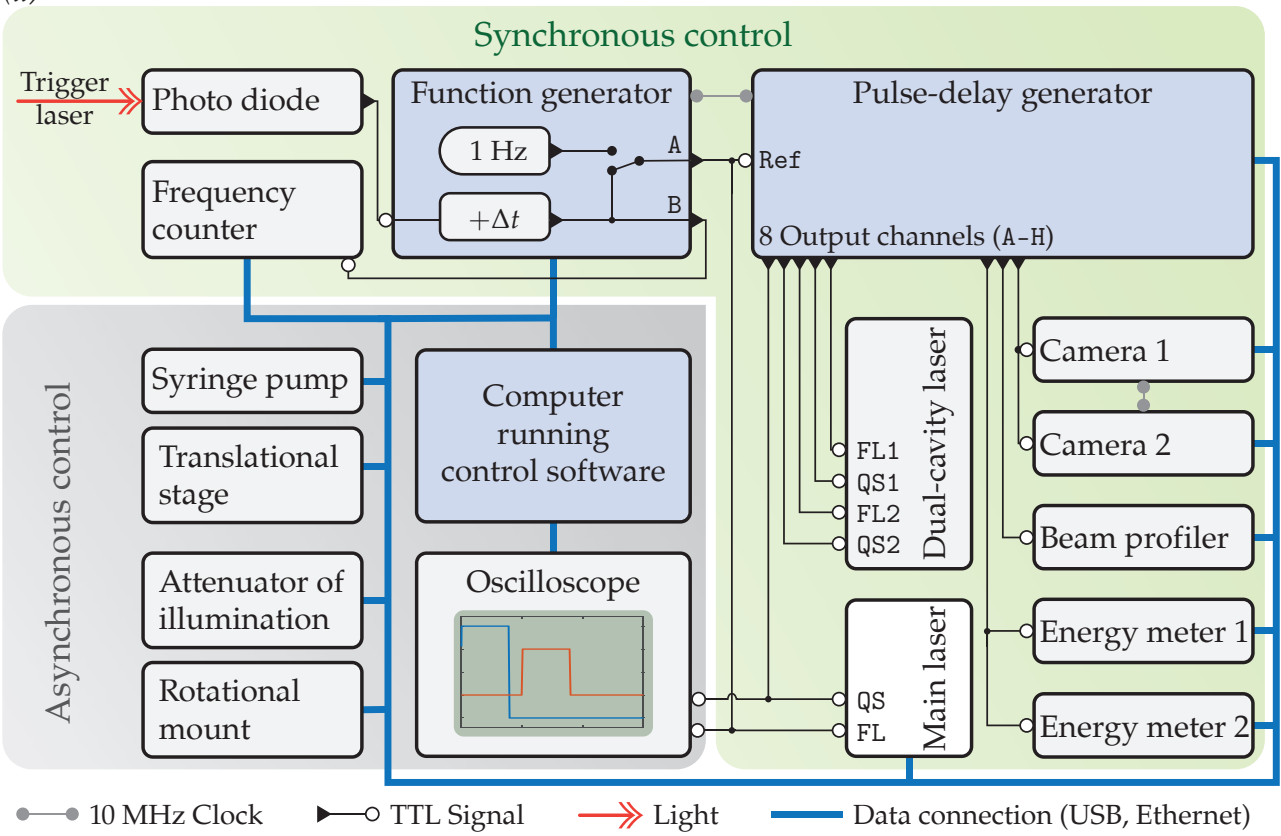

(b)

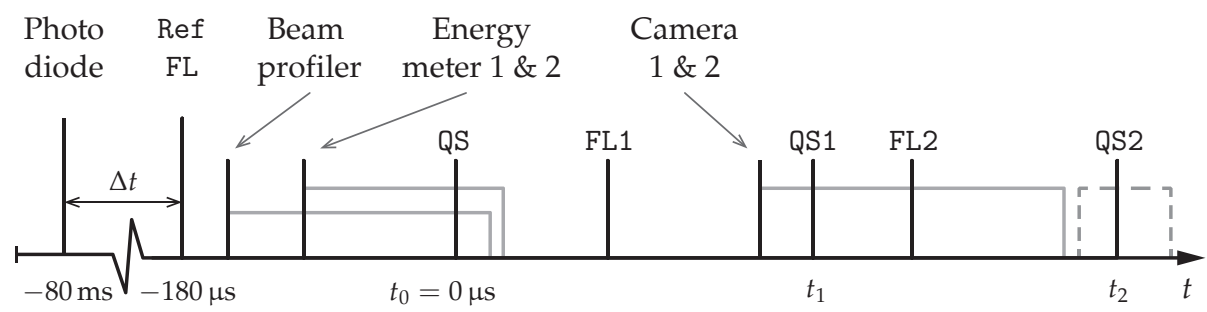

Figure 3.7: (a) Block diagram of the devices typically used in a laser-impact experiment. The diagram is split into two groups: the asynchronous group holds devices that are controlled on a timescale much longer than a single experiment and do not require a real-time synchronization. On the other hand, the synchronous control group consists of hardware that needs to interact with each other deterministically on a timescale down to a few nanoseconds. Our software to control devices in an object-oriented way in MATLAB and handle the data from the cameras or beam profiler is available online [73-75]. (b) Timing diagram of a stroboscopic experiment. Each vertical bar represents the rising edge of a trigger signal. In case the pulse width of a signal is of importance, either because a device needs a certain activation time (beam profiler and energy meters) or reacts also to the falling edge of the signal (camera 1 and 2), the complete pulse is shown as gray solid line. The dashed line is not an actual TTL signal but indicates the second exposure of the dual-frame camera, which is a response to the falling edge of its TTL signal. 
4. The pulse-delay generator is programmed to create appropriate trigger signals once it will be activated. Likewise, the energy meters and cameras are prepared by first flushing the onboard memory and followed by the initialization of a new data acquisition. Any motorized stage, such as the rotational mount of the $\lambda / 2$-plate to set $E_{\mathrm{L}}$, is moved into position.

5. The output of channel A of the function generator is synchronized to the trigger received from the photodiode (Fig. 3.7 shows this case). At this point the flash-lamp of the main laser and the actual experiment itself are synchronized to the drop generation. The setup is initialized and the generation of timing signals can then be started at any time.

6. The output of the pulse-delay generator is activated leading to an output of timing signals as illustrated in Fig. 3.7 (b). Each time a drop masks the photodiode a trigger signal is generated at $t=-80 \mathrm{~ms}$. A delay $\Delta t$ is added to that trigger by the function generator before it is passed to the pulse-delay generator as reference trigger Ref and to the flash lamp FL of the main laser. The flash-lamp signal FL needs to precede the actual output (activated by the Q-switch signal QS) of the main laser by about $180 \mu \mathrm{s}$. The moment the main laser receives QS defines the time of laser impact $t=0$, at which point the drop needs to be at the desired impact position. The alignment along $\vec{e}_{x}$ can be tuned by setting an appropriate $\Delta t$ (alignment along $\vec{e}_{y}$ and $\vec{e}_{z}$ is done mechanically, see $\S 3.6$ ). The beam profiler and energy meters require a certain activation time, which is why they receive a trigger signal before QS. The time an image is taken is set by the moment of exposure by the pulsed light source. In case the dual-cavity laser as described in $\S 3.5$ is used, the exposure of the first and second image is set by the Q-switch signal QS1 and QS2, respectively. Also here, the laser pumping by the corresponding flash-lamp needs to be activated before the Q-switch signal is received (about $135 \mu$ s for our laser system). It is important to note that the earliest possible time to generate an output for the pulse-delay generator is immediately after receiving trigger Ref, which leaves $180 \mu$ s before the laser impact to activate all other devices. Given the laser-specific timings this restriction allows us to take stroboscopic images starting at $t=-45 \mu \mathrm{s}$.

7. The experiment of the single-drop impact is repeated for as many drops as requested before the output of the pulse-delay generator is stopped. The back-view camera can be moved along $\vec{e}_{z}$ during the experiments to tune the image plane to the position of the drop in each frame. Where possible, the data acquired by the devices is already streamed during the experiment to a solid-state disk, but the control software checks after 
the trigger signals are stopped whether an equal number of records are acquired from all synchronized devices. The oscilloscope can be used during the experiment as live visualization to check the timing signals.

The triggering changes slightly when the high-speed cameras are used as they record for each experiment all events from the laser impact at $t=0 \mathrm{~s}$ to the late-time events at a fixed frame rate, typically $f_{\text {cam }}=10^{4} \mathrm{fps}$ at maximum image resolution. Therefore, the camera trigger is synchronized to the QS trigger (Fig. 3.7 (b)). However, this synchronization does not yet ensure that the first frame is actually exposed at $t=0 \mathrm{~s}$ as the internal clock of each highspeed camera is freely running leading to a maximum uncertainty in the frame time of $1 / f_{\text {cam }}=100 \mu \mathrm{s}$. In order to ensure proper synchronization to the laser impact we therefore reset the internal clock of each high-speed camera just before the laser pulse is emitted at $t=0 \mathrm{~s}$, also called a random-reset mode. This procedure then allows to take high-speed images with a precision of a few nanoseconds relative to the laser impact.

This section ends the description of the experimental apparatus. Our software to control devices in an object-oriented way in MATLAB and handle the data from the cameras and beam profiler is available online as open source [73-75]. We then used the setup to obtain the results presented in the next section: the stability of the control parameters of our experiment is discussed, followed by examples of the stroboscopic and high-speed imaging. 


\subsection{Results}

The experimental setup has been used at different stages of construction to acquire the data presented throughout this thesis for water and solvent drops. Examples for all visualization techniques described in this chapter can be seen in two award-winning videos (Fig. 3.8) for the side-view visualization.
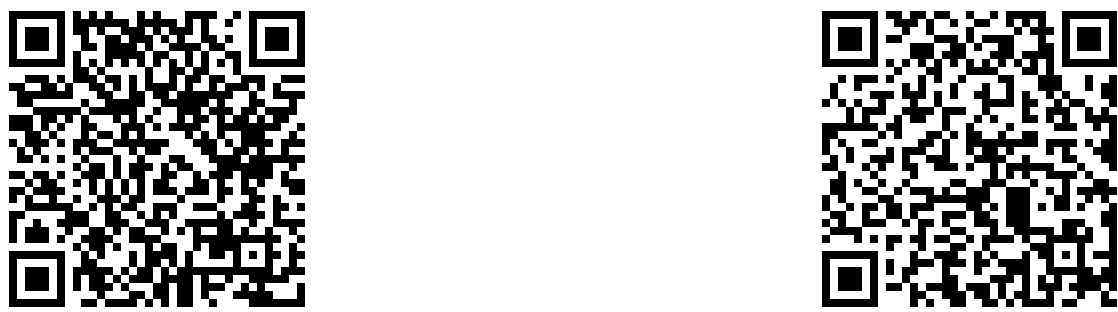

Figure 3.8: Scan a quick response code with your smartphone to watch the Miltonvan-Dyke winner of the Gallery at Fluid Motion 2014 at https : / www . youtube .com/ watch? $\mathrm{v}=\mathrm{bRbHDtPbHe} 0$ or the fine work winner from the Slow Motion Video Award 2016 at https : //www . youtube . com/watch?v=0Bv1 JBU2ENo

Here, we first show the stability of the control parameters of our experiment in §3.9.1 followed by a brief introduction to the laser impact on a drop based on the stroboscopic imaging in §3.9.2. Finally, we use the high-speed imaging in $\S 3.9 .3$ for experiments on how the fluid dynamics can be controlled by the laser-beam profile.

\subsubsection{Laser and drop stability}

The independent control parameters of our experiment are the spatial distribution of laser energy per unit area expressed as fluence $F(x, y, z)$, the liquid of the drop, and the initial radius $R_{0}$ and position of the drop in the lab reference frame. The liquid refers here to a specific solution that sets the absorption coefficient $\alpha$ and liquid properties such as density $\rho$ and surface tension $\gamma$, already explained in $\S 3.7$. These quantities characterize a specific laser-impact experiment and need to be known for each realization. A dependent quantity or outcome of the experiment, such as the propulsion speed of the drop $U$ due to the laser impact, can be inferred in the post-processing from the recorded data. The information combined leads finally to the relevant physical dimensionless parameters, such as the Weber number, that characterize the fluid-dynamic response in a more general way, independent of the actual experimental realization. Here, we focus on how we determine the independent control parameters and evaluate their stability over time, which is of great importance for the stroboscopic imaging. 
The beam focusing and imaging described in $\S 3.2$ are performed on a length scale in $\vec{e}_{z}$ large compared to the drop radius, e.g. $f_{5} / R_{0} \gg 1$. Therefore, the fluence $F(x, y, z)$ can be assumed to be constant as it propagates along $\vec{e}_{z}$ across the drop-impact location $-R_{0} \leq z \leq R_{0}$. The spatial distribution is imaged in relative terms $f(x, y, z=0)$ by the beam profiler as described in $\S 3.3$. The total energy of the laser beam $E_{\mathrm{L}}$ at the drop-impact location is inferred from an appropriate calibration of energy meter 1 . Both measurements combined allow to calculate the fluence in absolute terms

$$
\begin{aligned}
F(x, y, z=0) & =F_{0} f(x, y, z=0), \text { with } \\
F_{0} & =E\left(\int_{A} f(x, y, z=0) \mathrm{d} A\right)^{-1},
\end{aligned}
$$

where $A$ corresponds to an area on the CCD of the beam profiler large enough to cover the complete beam. Care must be taken to calibrate the base level of the beam profiler to exclude the noise in the integration of Eq. (3.4). To determine the size of the laser beam and the corresponding area $A$, we follow the ISO standard [76], our implementation is available online [74, 75].

A typical beam-profile is shown in Fig. 3.9 (a) obtained for the optical path from $\S 3.4$ including the near-field imaging and beam-shaping technique. The measurement is performed at low energy to avoid plasma generation in the intermediate focus at $z=30 \mathrm{~mm}$ (see Fig. 3.4). During each experiment the image recorded by the beam profiler can be used to determine the drop position and radius $R_{0}$ as shown in Fig. 3.9 (b). We obtain good results even though a plasma may be created in the intermediate focus behind the drop: the optical breakdown occurs close to the intermediate focus at $z>30 R_{0}$ and is not within the depth of field of the imaging optics of the beam profiler. We find a spread in $R_{0}$-values between measurements based on the beam-profiler image data and recordings of the side-view and back-view to be within $\pm 3 \%$. Combining the information of $R_{0}$, the drop position, and the fluence we can compute the energy transmitted into the drop per unit area (Fig. 3.9 (c)).

The beam profile as given in relative terms $f(x, y, z=0)$ is not a function of the total laser energy, which we confirmed for the relevant range $E_{\mathrm{L}, \min } \leq E_{\mathrm{L}} \leq E_{\mathrm{L} \text {,max }}$. It is therefore sufficient to measure the beam profile once for a given focusing or imaging condition and only measure the scalar quantity $E_{\mathrm{L}}$ for each experimental realization to compute the absolute scale according to Eq. (3.4). In case the near-field imaging is not used but the beam propagates out-of-phase to the drop-impact location, the spatial distribution is deteriorated as can be seen by comparison of Fig. 3.9 (a) and Fig. $3.12(a, b)$. As higher modes of the laser beam decay faster the superposition of all modes changes along $\vec{e}_{z}$. This illustrates the advantage of an imaging technique, where the modes in the conjugated focal plane preserve their phase relation. 


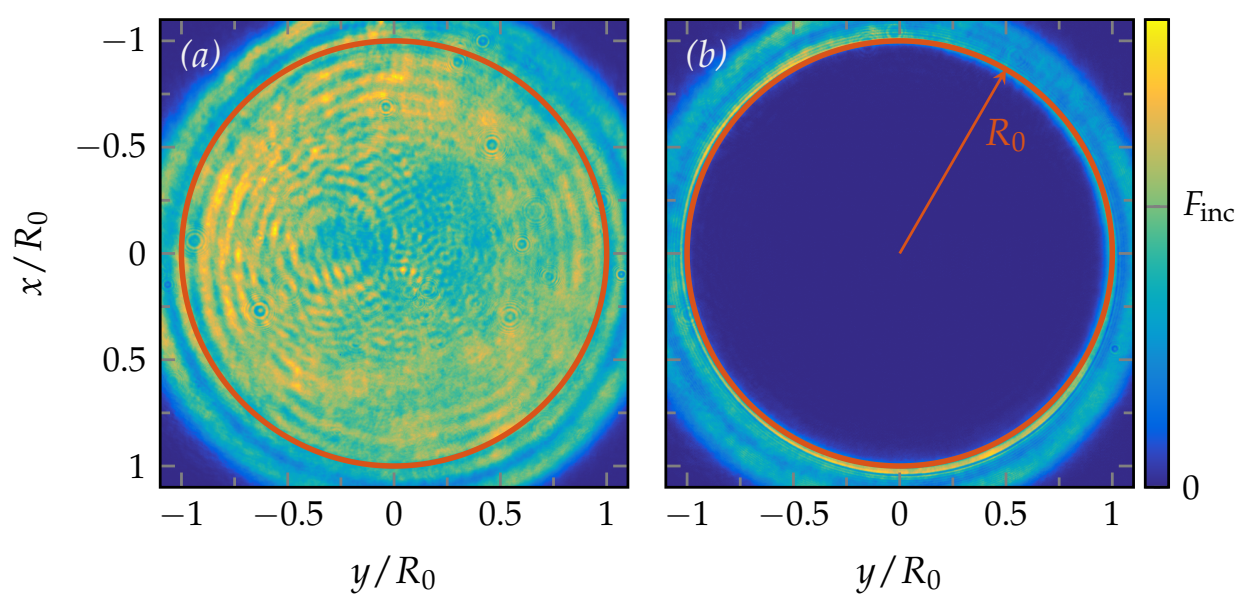

(c)

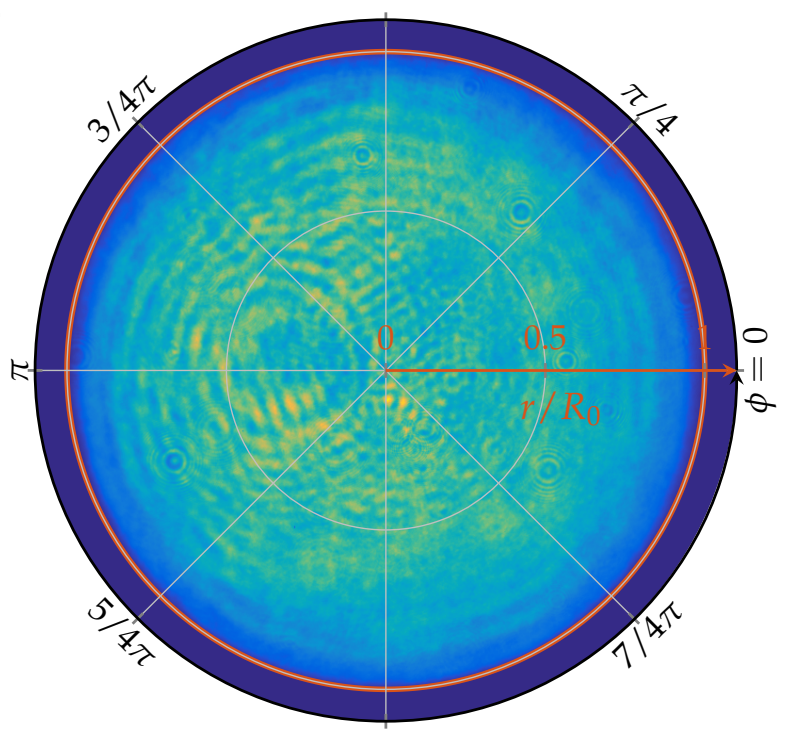

Figure 3.9: Laser-beam profile recorded in absence of the drop in (a) and recorded with the drop in (b). The solid line ( - ) in both images indicates the drop size and position determined from $(b)$. This information allows to calculate the energy per unit area $F_{\text {abs }}$ absorbed by the drop taking Fresnel reflection [65] at the liquid-air interface into account as shown in (c). The quantity $F_{\text {inc }}$ shown in the colorbar is the mean fluence in the part of the laser-beam that the drop is exposed to. 
The stability of the control parameters over time is shown in Fig. 3.10 for the same stroboscopic experiment as shown in Fig. 3.11. The liquid in this experiment is a MEK solution at about half the solubility limit and a laser energy $E_{\mathrm{L}}=113 \mathrm{~mJ}$ was chosen to be far above the fragmentation threshold. This operation point then creates a demanding test case for the experimental setup as many tiny fragments are created during each realization that could possible interfere with subsequent repetitions of the experiment. The control parameters of the experiment are stable over time as quantified by the standard deviation $\sigma$ obtained from a Gaussian fit to the distribution of each control parameter, see Fig. 3.10. To conclude, the setup allows to measure the control parameter for each realization of the experiment accurately and the shot-toshot variation of the control parameters is less than $3 \%$.

\subsubsection{Laser impact regimes}

The impact of a laser pulse on a liquid drop can lead to a violent response as already shown in Fig. 3.1: the liquid is set into motion on a timescale of a few microseconds, strongly deforms, and eventually fragments. The first reaction to the laser impact visible in the figure is the emission of a shock wave in the surrounding air (Fig. 3.1 (a) and Fig. $3.11(\mathrm{a}, \mathrm{c})$ ). The shock wave is followed by the ejection of matter in vapor and liquid state: the shock wave, visible as bright half-circle at the very left of Fig. 3.11 (c) is followed by a region filled with vapor, which is visible as Schlieren in the images. Still attached to the left of the drop is a mist cloud visible as a gray-to-black haze. Eventually, the drop propels forward in the direction of the laser propagation $\vec{e}_{z}$ as the vapor is ejected in opposite direction $-\vec{e}_{z}$, explained in more detail in $\S 4$. The center-of-mass speed of the drop that is induced in the example of Fig. 3.11 is approximately $U=10 \mathrm{~m} / \mathrm{s}$. It can be further increased as the maximum laser energy is not fully utilized in this example.

The color images in Fig. 3.1 are filtered by a notch filter centered at $532 \mathrm{~nm}$, which leads to the magenta colored background in the images. Nevertheless, the color camera resolves the visible spectrum of the light and helps to conclude on the driving mechanism of the fluid motion, i.e. whether a plasma generation and the corresponding plasma pressure needs to be taken into account. In case of a tightly focused beam (Fig. 3.1 (a)), which is realized by moving lens $\mathrm{f}_{5}$ (Fig. 3.2) such that the focal point of the lens is aligned with the drop, a white glow in the images reveals the generation of a plasma: the combination of different wavelengths in the visible spectrum appears white in the color images and indicates that the threshold for optical breakdown in the liquid is reached [77]. The plasma pressure then needs to be included in a 

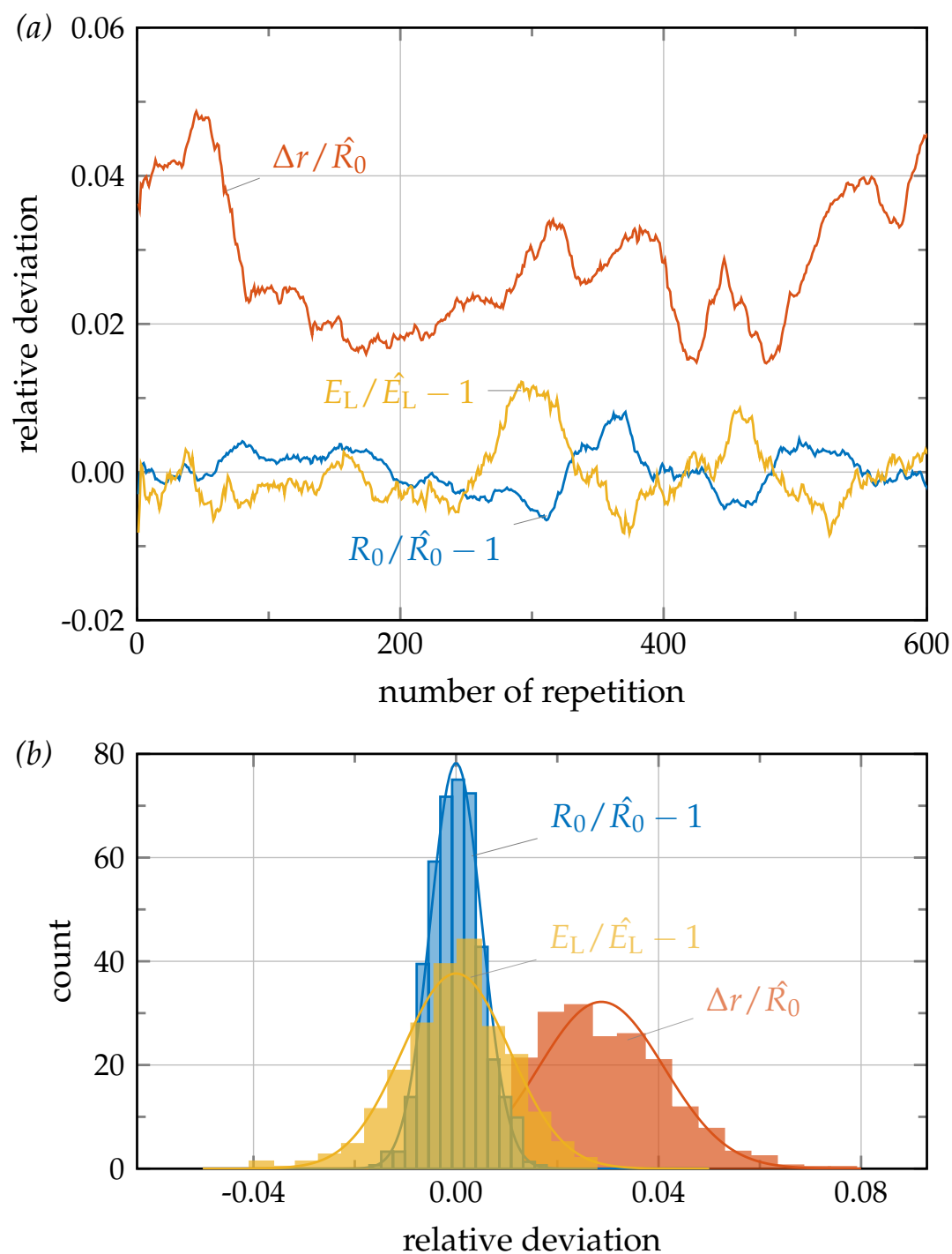

Figure 3.10: The relative deviation among 600 repetitions over the course of $10 \mathrm{~min}$ in (a) and as histogram in (b) for the laser energy $E_{\mathrm{L}}$, initial drop radius $R_{0}$, and the offset $\Delta r$ in the radial position of the drop. Each quantity is normalized by an appropriate mean value, either the mean initial radius $\hat{R}_{0}$ or the average laser energy $\hat{E}_{\mathrm{L}}$. The solid lines in (b) represent a Gaussian fit to each distribution yielding the standard deviation $\sigma$ for each control parameter: $\sigma_{E}=0.01, \sigma_{R_{0}}=0.005$ with an average relative offset in the drop position of $0.0287 \pm 0.0124$. The data is taken from the experiment shown in Fig. 3.11 and a running average filter is applied to the data in (a) for better visualization (statistics in (b) are based on the unfiltered data). 

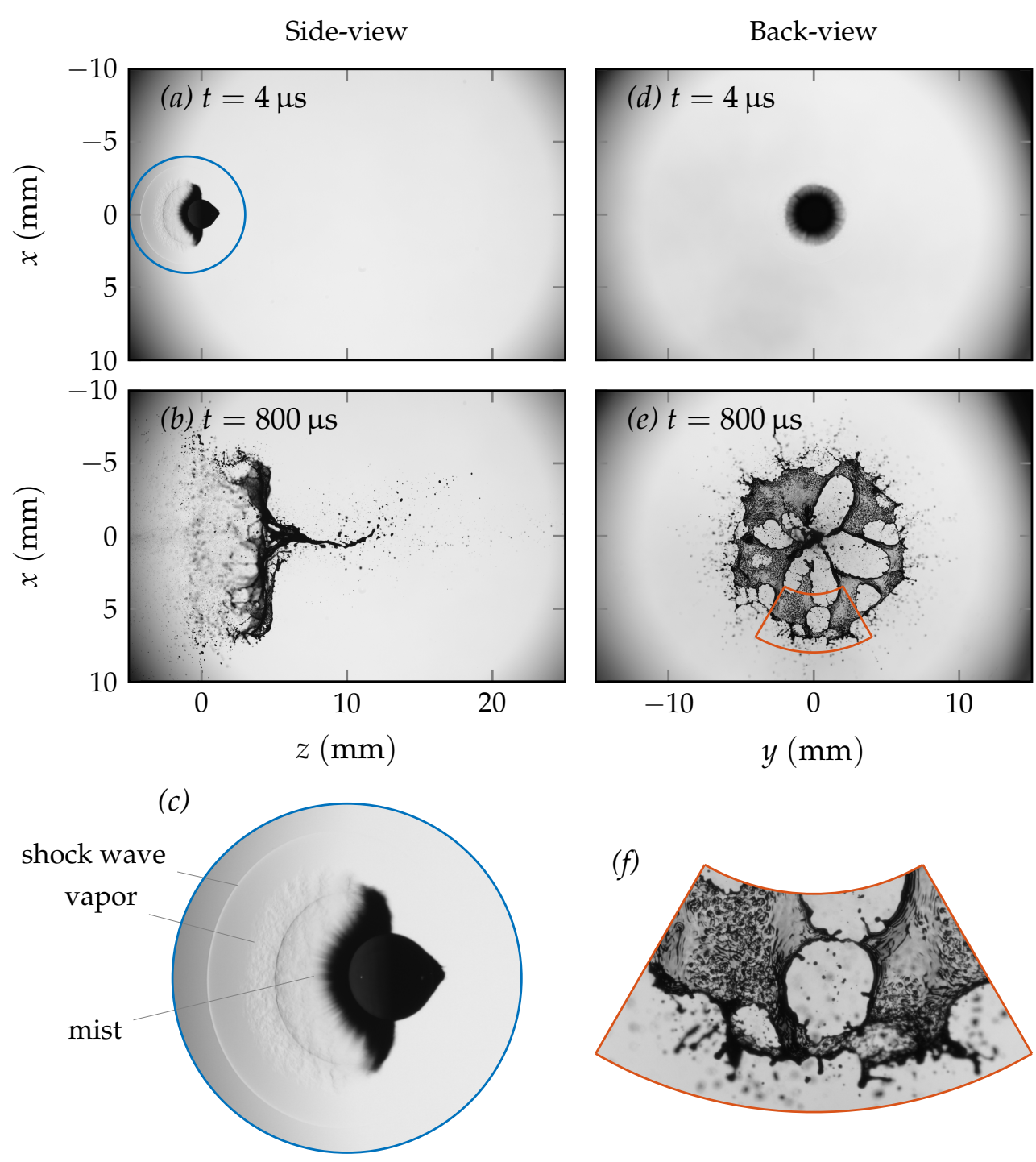

Figure 3.11: Side-view $(\mathrm{a}-\mathrm{c})$ and back-view $(\mathrm{d}-\mathrm{f})$ images taken stroboscopically at $t=$ $4 \mu \mathrm{s}(\mathrm{a}, \mathrm{c}, \mathrm{d})$ and $t=800 \mu \mathrm{s}(\mathrm{b}, \mathrm{e}, \mathrm{f})$ after laser impact. Images (c) and (f) are magnified regions of the full-frame images (a) and (e). The visualization is performed with the monochromatic and pulsed light source shown in Fig. 3.2. The laser energy per pulse is $E_{\mathrm{L}}=113 \mathrm{~mJ}$ and the liquid is Oil-Red-O dissolved in MEK at approximately half the solubility limit $\left(w=0.5 w_{s}\right)$. 
description of the driving force for the fluid motion. When the beam waist of the laser is moved away from the drop by moving lens $\mathrm{f}_{5}$ closer to the drop, the fluence at the drop location is decreased below the threshold for plasma formation in water of about 100 to $400 \mathrm{~J} / \mathrm{cm}^{2}$ [20]. Still captured by the camera is the fluorescence emission at a wavelength $\lambda>532 \mathrm{~nm}$ (visible as yellow spot in Fig. 3.1 (b, c)), which is caused by an emission from a resonantly excited state of the dissolved dye molecules. Local vaporization is the only driving mechanism of fluid motion in this case as the optical radiation pressure from the laser and the thermal radiation pressure caused by the heating of the drop surface are insignificant ${ }^{\dagger}[78]$, see also $\S 4$.

Care must be taken when interpreting the time an event occurs in stroboscopic images. All frames are exposed by a pulse of light less than $10 \mathrm{~ns}$ in duration at the time $t_{1}$ marked by QS1 in the timing diagram 3.7 (b). This pulse of light captures the fluid dynamics free of motion blur on the CCD of the camera at a well-known point in time. However, the camera is activated sufficiently earlier in time to account for any delay of the electronics or even a mechanical shutter in front of the CCD. Therefore, the CCD captures events also before and after $t_{1}$. In case of Fig. 3.1 this includes any plasma glow or fluorescent-light emission during and shortly after the laser pulse. Which means the camera is in fact exposed twice: once by the plasma or fluorescence light emission at $t=0 \mathrm{~s}$ and later at $t_{1}$ by the pulsed light source.

A clear advantage of stroboscopic imaging is seen in Fig. 3.11: events at an arbitrary point in time can be captured even though they can be apart by several orders of magnitude. At early times, the side-view gives insight in the propulsion mechanism of a drop upon laser impact (see Fig. $3.11(\mathrm{a}, \mathrm{c})$ ), whereas at late times details of the fluid dynamics such as corrugations on the evolving liquid sheet are revealed in Fig. $3.11(\mathrm{e}, \mathrm{f})$. The image resolution of $4008 \times 2672$ pixels per image is sufficient to resolve small details in an overall large FOV. The grid arrangement with a common axis for Fig. $3.11(a, b, d, e)$ is possible due to the image calibration. Each individual view is calibrated by its own set of calibration images and the location among the different views is calibrated by the position of the initial drop. Therefore, it is crucial to record the drop prior to laser impact to acquire an image of the drop free of any deformation. In that case, the spherical drop can be analyzed in the images of each view to find its center-of-mass position, which is then used as a reference for the calibration.

${ }^{\dagger}$ The typical impulse $I$ exerted on the drop by the 3\% reflected light from the surface scales as $I \sim E_{\mathrm{r}} / c$, with $E_{\mathrm{r}}$ the energy of the reflected light and $c$ the speed of light. This impulse would yield a typical drop speed $U \sim 10^{-7} \mathrm{~m} / \mathrm{s}$. The impulse due to thermal radiation from the hot drop surface scales as $I \sim \epsilon \sigma T^{4} R_{0}^{2} \tau_{p} / c$ with $\epsilon$ the emissivity and $\sigma$ the Stefan-Boltzmann constant. This impulse would yield $U \sim 10^{-14} \mathrm{~m} / \mathrm{s}$. 


\subsubsection{Controlling the fluid dynamics}

A key feature of the experimental setup is its capability to control and image the spatial distribution of laser radiation that drives the fluid dynamics. However, the timescale of laser radiation and drop dynamics are separated by several orders of magnitude. The question then arises: how reproducible is the late-time drop dynamics and can it be influenced by the early-time laser pulse? High-speed imaging plays an important role in the answer to such a question, since it does not require a reproducible process as in the case of stroboscopic imaging. Each individual feature can be followed in time for each realization of an experiment, only limited by the frame rate and image resolution. The two high-speed cameras allow to record images at a maximum resolution of $1024 \times 1024$ pixels at a frame rate of $f_{\text {cam }}=10000 \mathrm{fps}$.

To answer the question on the reproducibility of the drop dynamics we record ten realizations of a laser-impact experiment at constant control parameters. The laser-beam profile is shown in Fig. 3.12 (a) and exhibits an elliptical shape, i.e. we do not improve the shape by the beam shaping described in $\S 3.4$. The fluid-dynamic response to the laser impact can be observed in terms of the back-view visualization in Fig. 3.12 (c), which is a superposition of all ten realizations taken by the high speed recording at a fixed time. As can be seen, the shape evolution and even the position where ligaments are expelled radially outwards are reproducible features of the experiment: the gray value in the overlay image is a measure for the probability that ligaments occurred at the same position in all ten realization, where a black pixel means that in all experiments a ligament is found at a particular position. The advantage of high-speed imaging in this context is the ability to choose the point in time for the overlay in the post-processing after the experiment. Since the data of multiple recordings can be stored in the camera memory, all ten experiments can be recorded in direct succession within just ten seconds.

We now rotate the beam profile in Fig. 3.12 (a) by $\theta=45^{\circ}$ to obtain the beam profile shown in Fig. 3.12 (b). Following the same procedure as before we come to the same conclusion: the laser impact on a drop shows a remarkable reproducibility. But even more, a comparison of Fig. 3.12 (d) and (c) suggests that the drop dynamics follow the same rotation $\theta$ as set by the beam profile. This becomes clear when we overlay the two images, one corrected by the apparent rotation $\theta$, which is done in Fig. $3.12(\mathrm{e})$. The overlay reveals that the large-scale fluid dynamics is entirely controlled by the initial beam profile. This explains why the control and visualization of the beam profile is of great importance to our experiments: although only present very initially during the laser impact it determines the drop dynamics to a large extent and is the most important control parameter. 


$$
\theta=0^{\circ}
$$

(a)

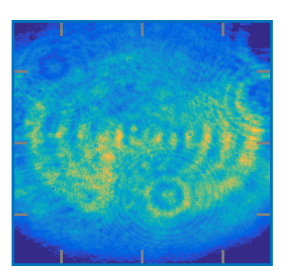

$\theta=45^{\circ}$

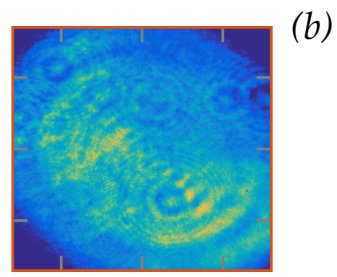

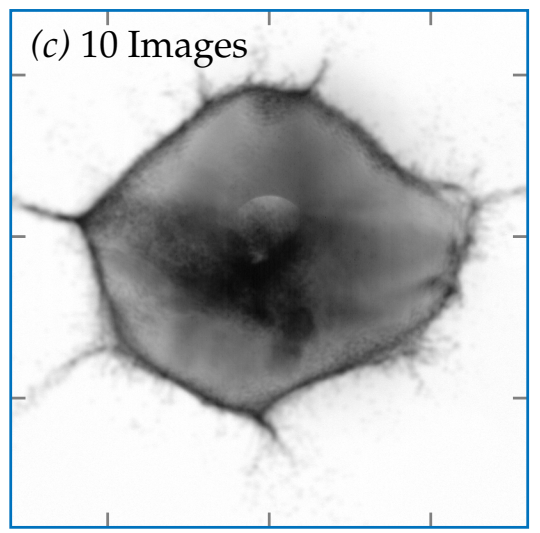

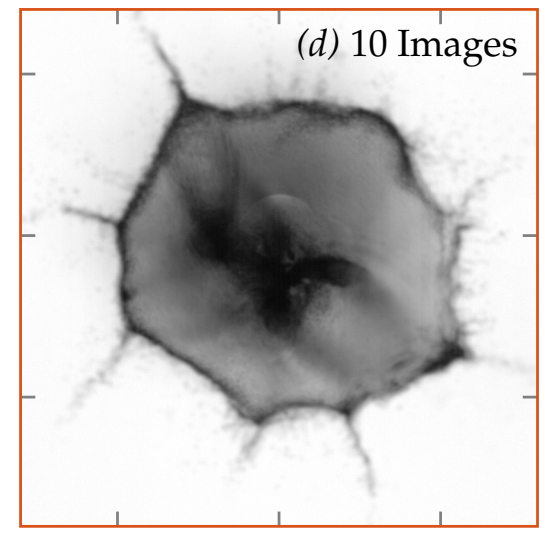

(e) 20 Images

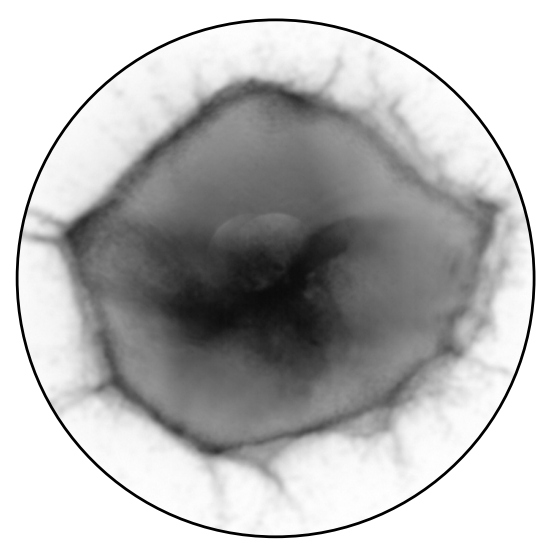

Figure 3.12: The influence of the laser-beam profile onto the fluid dynamics of the drop illustrated for two laser-beam profiles: a reference beam at a rotation about $\vec{e}_{z}$ of $\theta=0^{\circ}$ in (a) and a rotated beam at $\theta=45^{\circ}$ in (b). Ten experiments are recorded with a high-speed camera for each rotation $\theta$ and the superimposed frames taken at $t=900 \mu$ s are shown in (c) and (d). The image (e) is the superposition of (c) and (d), where (d) as been rotated by $-45^{\circ}$ to compensate the initial rotation of the laser-beam profile. The liquid in this experiment is an aqueous Acid-Red-1 solution. 


\subsection{Summary}

An experimental apparatus to control and visualize the fluid-dynamic response of a liquid target to a laser-induced phase change is presented. The laser is a Q-switched Nd:YAG laser system emitting pulses with a duration of $\tau_{p}=5 \mathrm{~ns}$ at a wavelength of $\lambda_{\mathrm{L}}=532 \mathrm{~nm}$ and a maximum energy of $E_{\mathrm{L}, \max }=420 \mathrm{~mJ}$. We explain two optical arrangements to hit the liquid target of interest. First, an optical path is shown that focuses the freely-propagating beam onto the target. The advantages of this arrangement are its simplicity and options to extend the path easily. Second, an optical path to image the beam profile from the near-field of the laser onto the liquid target is explained in detail. The path is more difficult to implement but can be combined with a beam-shaping technique. The beam shaping that we present allows for any linear combination of the laser beam with a rotated version of itself, which is used to improve the axi-symmetry of the beam profile.

A liquid drop with an initial radius $R_{0} \approx 1 \mathrm{~mm}$ is generated as target for the impact and we present two liquid-dye solutions that allow to tune the penetration depth of laser light into the drop in terms of a linear absorption coefficient $\alpha$ over a large range, i.e. $5 \times 10^{-5} \leq R_{0} \alpha \leq 400$. The response of the drop to the laser impact is visualized by high-speed and stroboscopic imaging in two orthogonal views: a side-view perpendicular to the laser beam and a back-view that is along the laser-beam propagation. The combination of imaging techniques with exposure times down to $10 \mathrm{~ns}$ resolves all relevant timescales that vary by orders of magnitude between the nanosecond duration of the laser impact and the millisecond evolution of the fluid-dynamic response.

The setup enables an operation at constant control parameters with a shotto-shot variation of less than $3 \%$, which makes stroboscopic imaging possible in the first place. We present examples from the laser impact on a drop that require this stability, foremost a case where the control over the beam profile allows perfect control over the late-time fluid dynamics. 


\section{4}

\section{Vapor propulsion of absorbing millimeter-sized drops ${ }^{*,+}$}

We show how the deposition of laser energy induces propulsion and strong deformation of an absorbing liquid body. Combining high speed with stroboscopic imaging, we observe that a millimeter-sized dyed water drop hit by a millijoule nanosecond laser pulse propels forward at several meters per second and deforms until it eventually fragments. The drop motion results from the recoil momentum imparted at the drop surface by water vaporization. We measure the propulsion speed and the timedeformation law of the drop, complemented by boundary-integral simulations. The drop propulsion and shaping are explained in terms of the laser-pulse energy, the drop size, and the liquid properties. These findings are, for instance, crucial for the generation of extreme ultraviolet light in nanolithography machines.

*Published as: Alexander L. Klein, Wilco Bouwhuis, Claas Willem Visser, Henri Lhuissier, Chao Sun, Jacco H. Snoeijer, Emmanuel Villermaux, Detlef Lohse, and Hanneke Gelderblom. Drop Shaping by Laser-Pulse Impact. Physical Review Applied, 3(4):044018, 2015.

${ }^{\dagger}$ The experimental work in this chapter is part of the present thesis. The numerical simulations are due to Wilco Bouwhuis. 


\subsection{Introduction}

A laser-induced phase change in liquids can lead to a violent response: deformation and disruption of the liquid body followed by the ejection of matter. The complete vaporization or even explosion of micrometer-sized drops can result from the linear absorption of laser energy [26-28]. Self-focusing and dielectric breakdown may lead to plasma formation in transparent drops [2932]. Laser impact has been used to generate liquid motion by vaporization or plasma formation in confined geometries [33-35], sessile drops [36], and biological matter $[20,37,38]$.

Here, we show how the absorption of laser energy by an unconfined liquid drop induces a rapid phase change (see Fig. 4.1), which in turn controls the propulsion, expansion, and fragmentation of the drop. A key application of the drop shaping by laser impact is found in laser-produced plasma light sources for extreme ultraviolet (EUV) nanolithography $[15,17]$. In these sources the shape, position, and stability of a liquid tin body directly affect the conversion efficiency of liquid tin to a plasma that emits EUV light.

The detailed understanding of the hydrodynamic response of an opaque liquid drop to a laser impact poses two fundamental challenges. First, one needs to resolve how momentum is transferred from the laser to the drop. Second, the subsequent deformation dynamics and fragmentation of the drop after impact have to be quantified. Although drop impact onto a solid substrate has been studied thoroughly (for a selection, see e.g. Ref. [42-48]), no consensus on the deformation dynamics has yet been reached and only few studies $[42,45,49,79,80]$ focused on the fragmentation.

\subsection{Experimental methods}

Our model system consists of a highly-absorbing drop that is hit by a pulsed laser beam. In Fig. 4.2 an overview of the experimental setup is shown. The drop detaches from a capillary, falls, and relaxes to a spherical shape with radius $R_{0}=0.9 \mathrm{~mm}$. While it falls down, the drop masks a photodiode that then generates a reference trigger for the pulsed laser, cameras, and light source. The $\vec{e}_{z}$-axis of the laser beam is aligned orthogonally to the $\vec{e}_{x}$-axis defined by the falling drop and the $\vec{e}_{y}$-axis of the imaging optics. The $y z$-plane in which the laser beam propagates is below the plane of the trigger laser and the pinch-off point at the capillary tube.

The drop consists of dyed water with a density $\rho=998 \mathrm{~kg} / \mathrm{m}^{3}$ and surface tension $\gamma=72 \mathrm{mN} / \mathrm{m}$ assumed to be equal to the properties of pure water. The typical penetration depth of the laser light into the dyed drop is $\delta \sim$ 

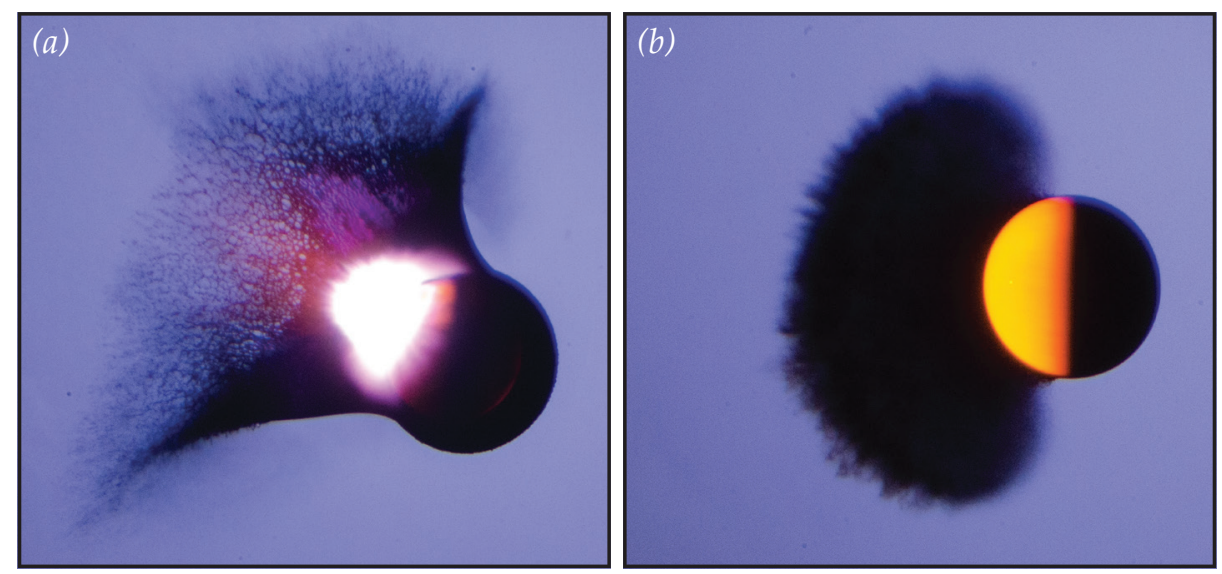

Figure 4.1: Laser pulses $\left(\lambda_{\mathrm{L}}=532 \mathrm{~nm}\right)$ impacting from the left on magenta-dyed water drops of radius $R_{0}=0.9 \mathrm{~mm}$. Images are taken $30 \mu \mathrm{s}$ after impact with a color camera and diffusive backlight illumination. (a) White plasma glow [77] and violent ablation from the drop induced by a focused laser beam. (b) Fluorescence of the dye and ablation at the drop surface due to local boiling induced by a uniform laser irradiation.

$10 \mu \mathrm{m} \ll R_{0}$ [33], which ensures that the laser energy is absorbed in a thin layer close to the drop surface. The laser-pulse energy is varied between $0 \mathrm{~mJ}$ and $120 \mathrm{~mJ}$ by an optical attenuator based on a half-wave plate and a polarizing beam-splitter. The relation between the laser-pulse energy at the drop location and the settings of the attenuator is determined in separate measurements, for which the top beam dump shown in Fig. 4.2 is replaced by an energy meter. A focusing lens decreases the beam diameter to twice the drop size in order to achieve a uniform but high-intensity illumination of the drop. To ensure the drop is placed at the center of the laser beam, the drop position is optimized such that the drop-shape evolution is axisymmetric with respect to $\vec{e}_{z}$ and the propulsion speed is maximum.

The energy $E_{\mathrm{abs}}$ that is actually absorbed by the drop is computed from a beam-profile measurement and ray-tracing, see appendix $\S 4$.A. The typical beam fluence $1 \mathrm{~J} / \mathrm{cm}^{2}$ is well below the dielectric breakdown and self-focusing thresholds reported for water with focused nanosecond laser pulses [35, 77]. Consistently, we observe a plasma only when the laser beam is tightly focused inside the drop (Fig. 4.1(a), see also Ref. [20,37]), but not for a uniform irradiation (Fig. 4.1 (b)). To visualize the wavelengths in the visible spectrum that are emitted by the drop shown in Fig. 4.1 we use a magenta-colored ink as a dye and a color camera. For all experiments leading to quantitative results we use a black-colored ink to suppress fluorescence. 


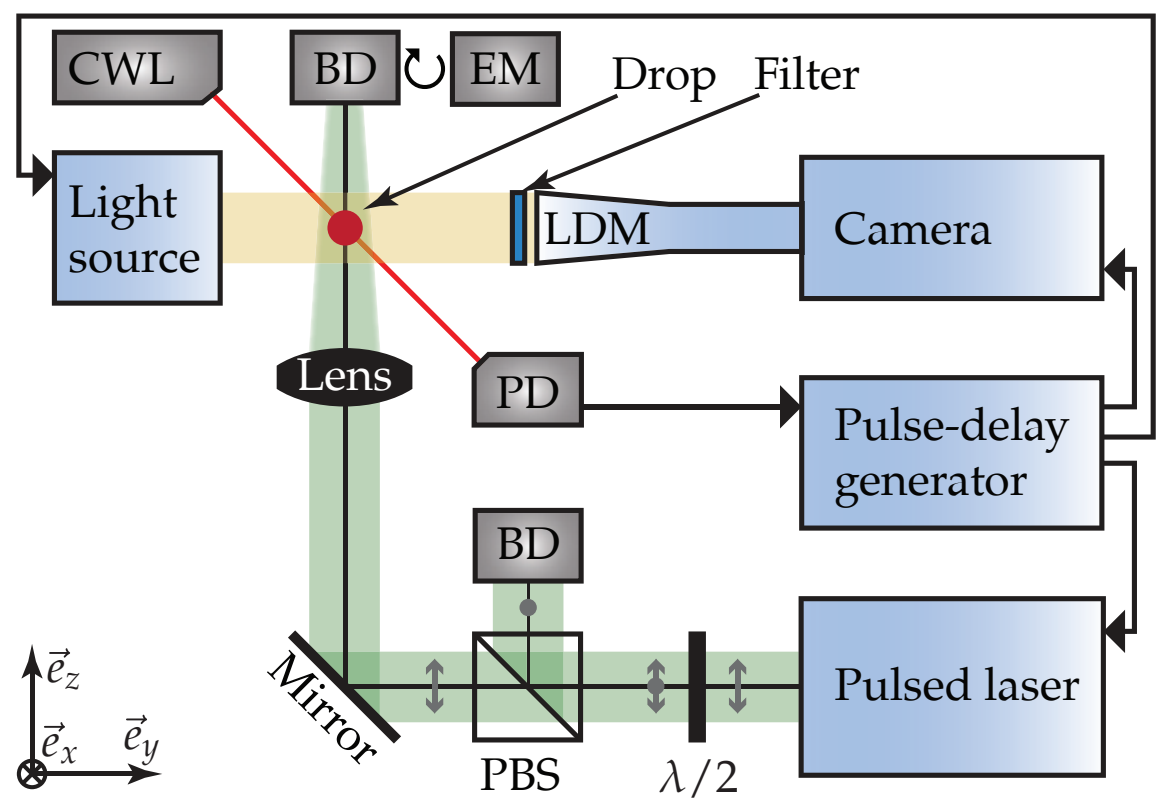

Figure 4.2: Sketch of the experimental setup in top view. The drop (black/magenta ink, IJC-5900/5920 by Sensient) is generated with a capillary tube connected to a syringe pump (PHD2000 by Harvard Apparatus, not shown). A continuous-wave laser (CWL, CPS196 by Thorlabs) and a photodiode (PD, PDA36A by Thorlabs) serve as light barrier to trigger on the falling drop. The lab equipment is synchronized by a high-precision pulse-delay generator (BNC575 by Berkeley Nucleonics) according to the indicated signal path. The pulsed laser is a frequency-doubled Nd:YAG laser (Evergreen 140 by Quantel) with a pulse duration $\tau_{p}=10 \mathrm{~ns}$ emitting at a wavelength $\lambda_{\mathrm{L}}=532 \mathrm{~nm}$. Attenuation of the laser energy is accomplished by a zero-order halfwave plate $(\lambda / 2)$, a polarizing beam splitter (PBS), and a beam dump (BD). The laser-pulse energy is measured by an energy meter (EM, QE12 by gentec-eo). The circle $(\bullet)$ and arrow symbols $(\leftrightarrow)$ along the optical axis respectively indicate the $S$ and P-component of the linearly-polarized laser beam that is focused by a planoconvex lens with a focal length of $\mathrm{f}=125 \mathrm{~mm}$. Side-view images ( $x z$-plane) are taken with a long-distance microscope (LDM, 12x Zoom by Navitar), a high-speed camera (FASTCAM SA-X2 by Photron), and a continuous light source (LS-M352A by Sumita). Stroboscopic images are acquired by a CCD camera (PCO1300 by PCO AG) combined with a light source (NANOLITE KL-K by HSPS) that delivers a high-intensity light pulse of $8 \mathrm{~ns}$. A notch filter protects the imaging equipment from scattered laser light. 
The post-impact dynamics of the drop (Fig. 4.3 (a)) is observed from a sideview ( $\vec{e}_{y}$-axis in Fig. 4.2) with a long-distance microscope, a high-speed camera operated at a frame rate of 20000 frames per second and a continuous light source. Detailed information in the first microseconds after impact is obtained by operating a camera in stroboscopic mode with a flash lamp that delivers a high-intensity light pulse of $8 \mathrm{~ns}$ (Fig. 4.3 (b)). We record stroboscopic videos by performing a single impact experiment per video frame while changing the time delay between the laser impact and the pulsed light source. For both cameras used the size of the field of view is $16 \times 10 \mathrm{~mm}^{2}$, which yields a pixel resolution of $16 \mu \mathrm{m}$ per pixel.

\subsection{Results \& interpretation}

The drop dynamics for different pulse energies is shown in Fig. 4.3. On impact, the surface of the drop hit by the laser emits a shock wave into the air (Fig. 4.3 (b)). The shock wave is followed by the ejection of a mist cloud of small drops that is visible as a gray-to-black haze in the images and persists for several microseconds. Subsequently, the mist is expelled while the drop propels in the opposite direction (Fig. 4.3(a)). At the same time the drop flattens and expands in the radial direction before it either retracts, for low pulse energy, or fragments, for large energy.

We quantify the drop motion by measuring the displacement $Z(t)$ of the drop center-of-mass and the drop radius $R(t)$ (defined in Fig. 4.3 (a)) for the first milliseconds after impact. As Fig. 4.4 (a) shows, the drop is propelled at a constant speed $U$ that increases with increasing pulse energy up to $2.0 \mathrm{~m} / \mathrm{s}$. The accompanying deformation of the drop occurs on the inertial timescale $\tau_{i}=R_{0} / U \sim 10^{-4}$ to $10^{-3} \mathrm{~s}$ (Fig. $4.4(\mathrm{~b})$ ) and is eventually slowed down by surface tension on the capillary timescale $\tau_{c}=\left(\rho R_{0}^{3} / \gamma\right)^{1 / 2}=3.5 \mathrm{~ms}$. Both the initial deformation rate $\tau_{i}^{-1}$ and the maximal extension $R_{\max }$ increase with increasing pulse energy. We emphasize the clear separation of timescales

$$
\tau_{p} \ll \tau_{e} \ll \tau_{i}<\tau_{c}
$$

between the successive steps, namely, the laser pulse, the ejection of matter (on timescale $\tau_{e} \sim 10^{-5} \mathrm{~s}$ ), the initial deformation of the drop, and its capillary retraction.

To explain the relation between the drop propulsion speed, the radial expansion, and the laser energy one needs to understand the mechanism that propels the drop. Surely, both the optical radiation pressure from the laser and the thermal radiation pressure caused by the heating of the drop 


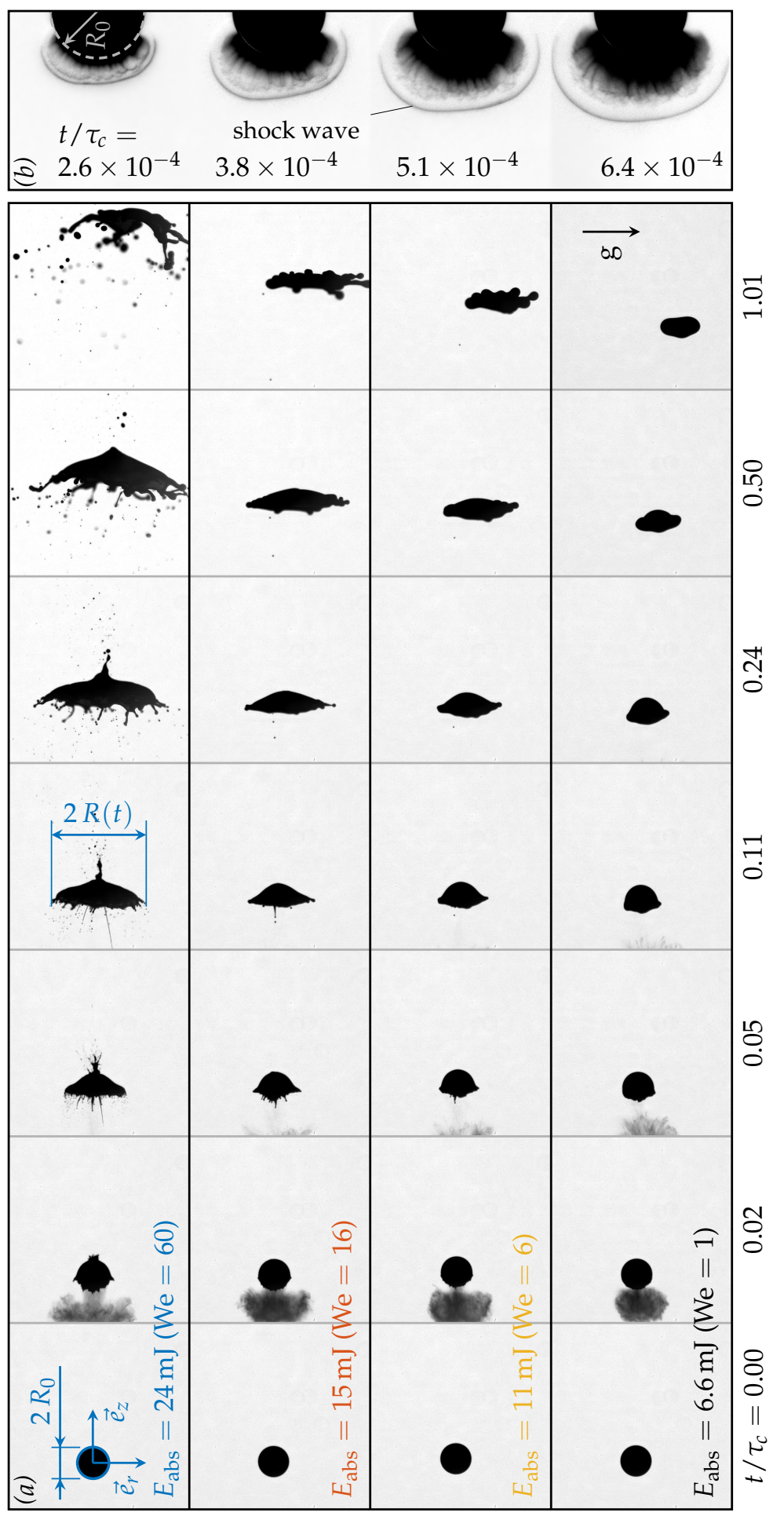

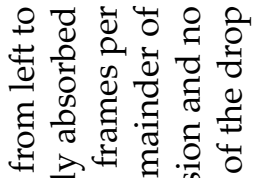

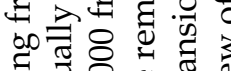

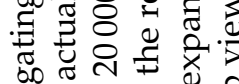
क.

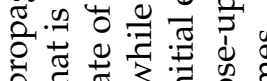

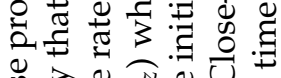

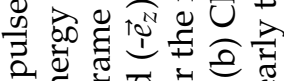

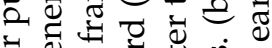

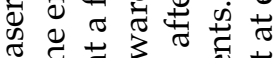

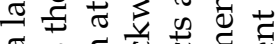
․․ ก क की 11 $\|$ केष ठ

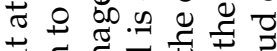

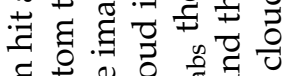

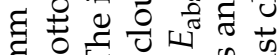
范 ठे $\|$ औ क क क क

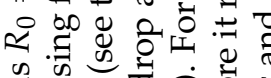
क के

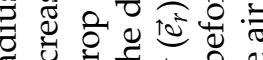

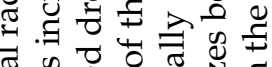

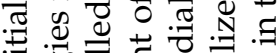
द.

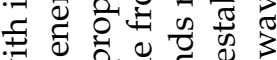
3 \& ㅇ. 윰 0 त 0 क क

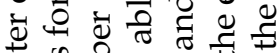

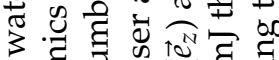

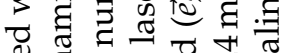
¿

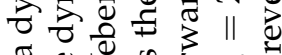

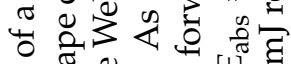

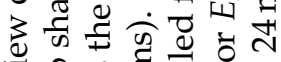

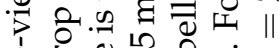

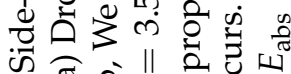
ஸे के

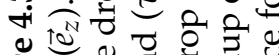

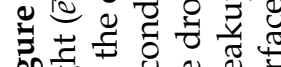

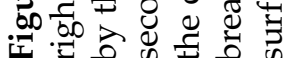



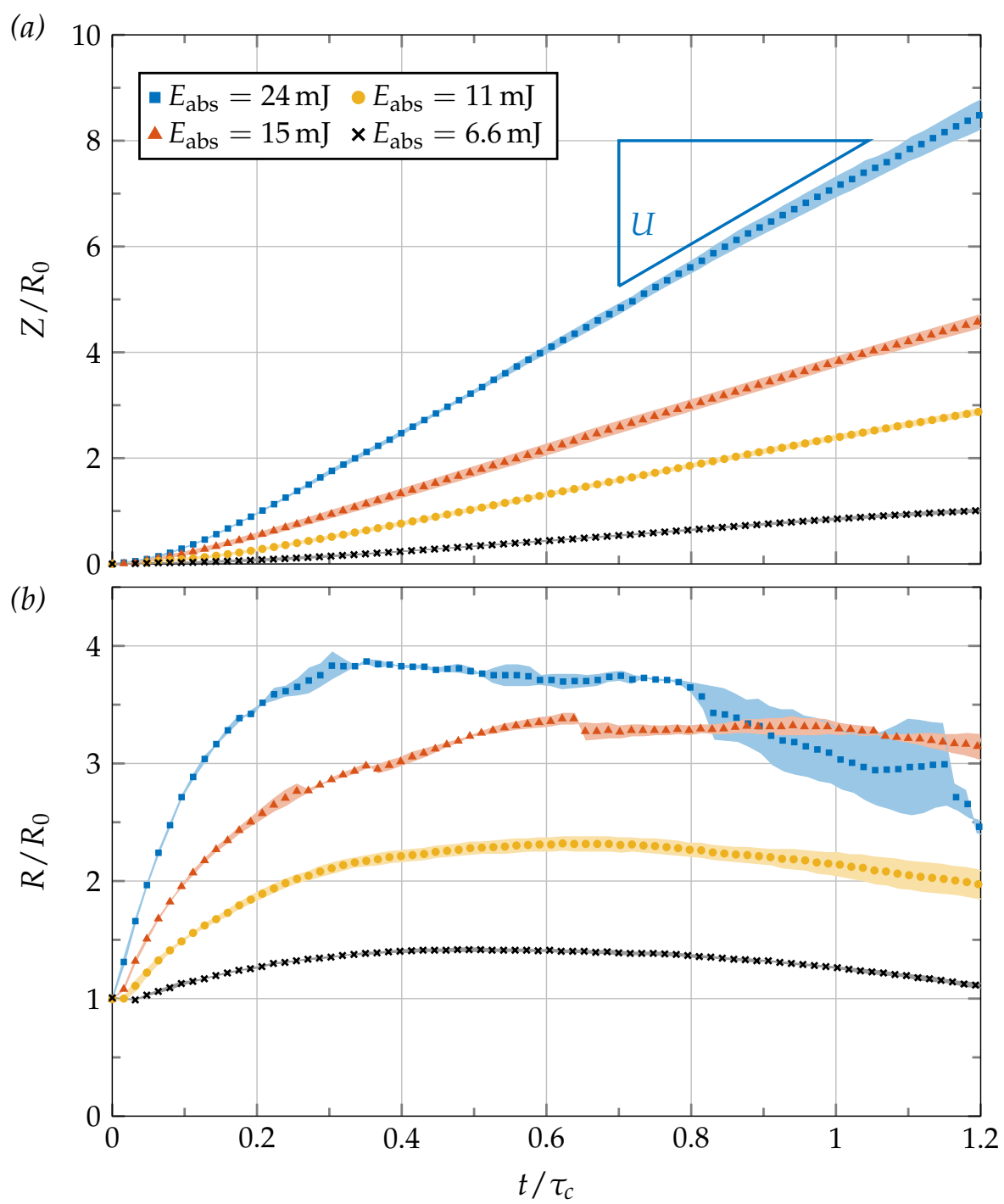

Figure 4.4: Center-of-mass displacement $Z$ (along $\vec{e}_{z}$, see Fig. 4.3) (a) and radial expansion $R(\mathrm{~b})$ as a function of time for different absorbed energies. The corresponding image sequences are shown in Fig. 4.3. Each point is averaged over two experiments and the shaded area indicates the difference between the two. The apparent acceleration in $Z$ for $t / \tau_{c}<0.2$ is an artifact of the method used to determine the center-of-mass position, see appendix $\S 4$.B. The large deviation in $R$ for $E_{\mathrm{abs}}=24 \mathrm{~mJ}$ illustrates the statistical nature of the fragmentation. For $E_{\mathrm{abs}}=15 \mathrm{~mJ}$ events of drop ejection from the edge are visible at $t / \tau_{c}=0.24,0.36$ and 0.63 . 
surface are insignificant ${ }^{\ddagger}$ [78]. The motion actually results from the recoil due to the partial vaporization of the drop: since the highly-absorbent dye ensures that the laser energy is absorbed in a superficial layer on one side of the drop, the vapor expulsion is mainly unidirectional and consequently transfers momentum to the remainder of the drop.

The light energy is absorbed by a liquid mass $\sim \rho R_{0}^{2} \delta$ set by the penetration depth of the laser. On the timescale $\tau_{e}$, both diffusive and radiative heat transfers are negligible (the thermal diffusion length is much smaller than $\delta)^{\S}$. Since the beam profile is flat, and neither the focusing due to the drop interface curvature nor nonlinear optical effects (self-focusing or electric breakdown) are significant, we consider the energy deposition in the superficial layer to be close to uniform. This energy is sufficient to heat the liquid from the ambient temperature $T_{0}=293 \mathrm{~K}$ to the boiling temperature $T_{\mathrm{b}} \simeq 393 \mathrm{~K}$, but not to vaporize all of it: only a certain fraction $\beta$ actually vaporizes. The energy balance therefore reads $E_{\mathrm{abs}} \sim \rho R_{0}^{2} \delta\left[c_{\mathrm{V}}\left(T_{\mathrm{b}}-T_{0}\right)+\beta \Delta H\right]$, where $c_{\mathrm{v}}=4.0 \mathrm{~kJ} /(\mathrm{kg} \mathrm{K})$ and $\Delta H=2.25 \mathrm{MJ} / \mathrm{kg}$ are, respectively, the specific heat capacity and latent heat of vaporization of the liquid.

In all our experiments a mist cloud is observed, which is a clear signal of a local boiling of the drop. We therefore assume that to get propulsion, a threshold energy $E_{\mathrm{th}} \sim \rho R_{0}^{2} \delta c_{\mathrm{v}}\left(T_{\mathrm{b}}-T_{0}\right) \approx 3 \mathrm{~mJ}$ has to be absorbed by the superficial layer to heat the liquid to the boiling point, which is in good agreement with the threshold for propulsion observed in our experiments (Fig. 4.5 (a)). Any additional energy deposited in the superficial layer is used to vaporize a mass of liquid $m \sim \beta \rho R_{0}^{2} \delta \sim\left(E_{\mathrm{abs}}-E_{\mathrm{th}}\right) / \Delta H$. An upper limit for the proposed scaling is given by $E_{\mathrm{abs}} / E_{\mathrm{th}} \sim 1+\Delta H /\left[c_{\mathrm{v}}\left(T_{\mathrm{b}}-T_{0}\right)\right] \approx 8$, in which case the absorbed energy is sufficient to evaporate the entire heated liquid layer (i.e. $\beta=1$ ). Any increase in $E_{\mathrm{abs}}$ beyond this point would lead to a superheated or even a critical phase, in which case the opaque mist cloud would not be observed [37].

For $0<\beta<1$, which is the case of our experiments, the remaining part of the heated layer that is not vaporized is expelled as a mist of small drops. We assume that the liquid vaporizes at $T_{\mathrm{b}}$ and that the vapor is expelled at the thermal speed $u=\sqrt{k_{\mathrm{B}} T_{\mathrm{b}} / \mu} \approx 400 \mathrm{~m} / \mathrm{s}$, where $k_{\mathrm{B}} \simeq 1.38 \times 10^{-23} \mathrm{~J} / \mathrm{K}$ is the Boltzmann constant and $\mu=2.99 \times 10^{-26} \mathrm{~kg}$ is the molecular mass of

\footnotetext{
‡The typical impulse $I$ exerted on the drop by the 3\% reflected light from the surface scales as $I \sim E_{\mathrm{r}} / c$, with $E_{\mathrm{r}}$ the energy of the reflected light and $c$ the speed of light. This impulse would yield a typical drop speed $U \sim 10^{-7} \mathrm{~m} / \mathrm{s}$. The impulse due to thermal radiation from the hot drop surface scales as $I \sim \epsilon \sigma T^{4} R_{0}^{2} \tau_{p} / c$ with $\epsilon$ the emissivity and $\sigma$ the Stefan-Boltzmann constant. This impulse would yield $U \sim 10^{-14} \mathrm{~m} / \mathrm{s}$.

$\S$ On the timescale of vapor ejection $\tau_{e}$ heat typically diffuses over a length $\sqrt{\kappa \tau_{e}} \sim 10^{-6} \mathrm{~m} \ll$ $\delta$, where $\kappa$ is the thermal diffusivity of water.
} 

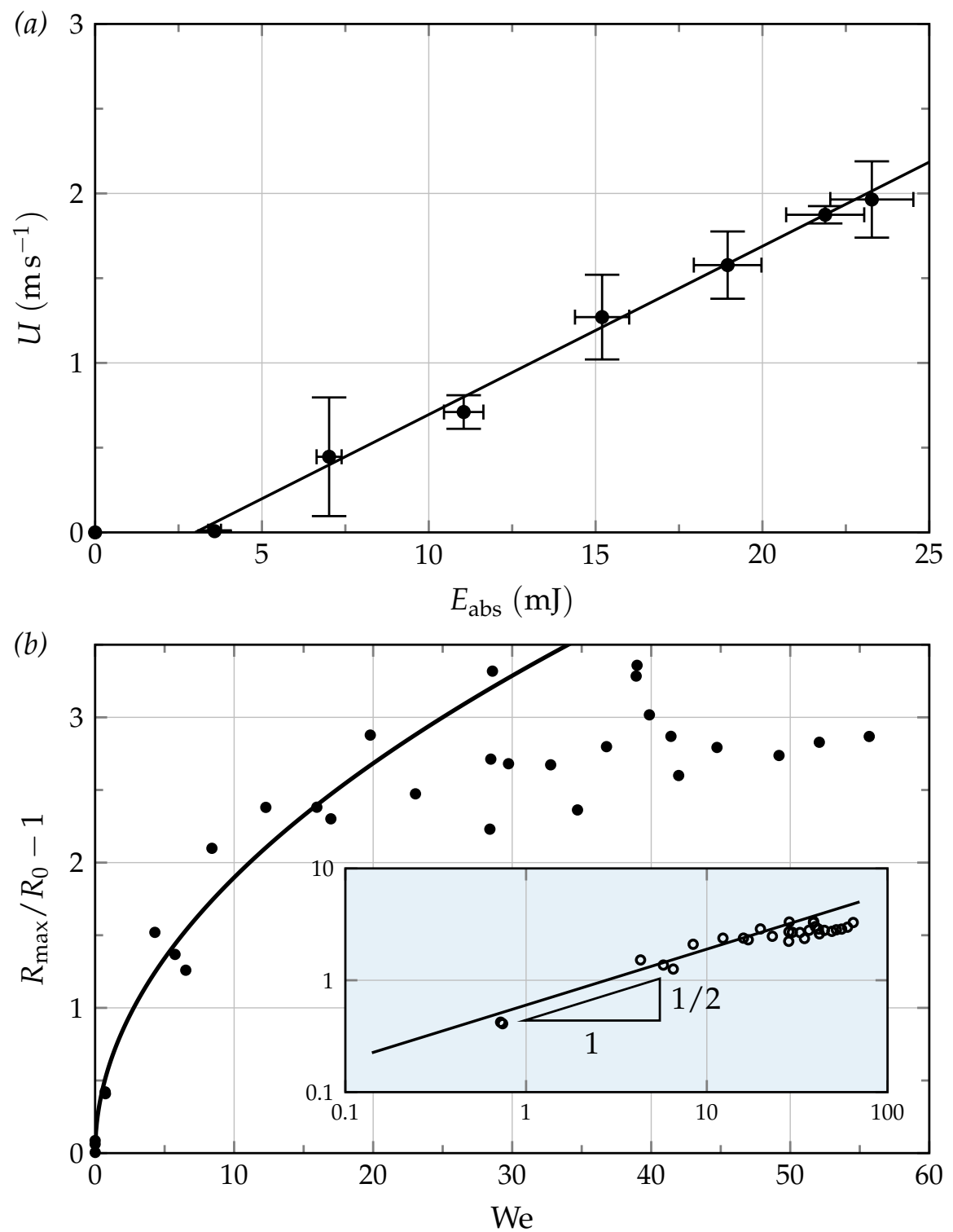

Figure 4.5: (a) Propulsion speed of the drop as a function of the absorbed laser energy. Each point represents at least four experiments, the error bars indicate the standard deviation. The solid line is equation (4.2) with a prefactor of 0.4. (b) Maximal relative expansion $R_{\max } / R_{0}-1$ for individual experiments as a function of the Weber number in linear and logarithmic (inset) scales. The solid line is equation (4.3) with a prefactor of 0.6. For large Weber numbers a saturation is observed due to the fragmentation of the sheet. 
water. This expelled vapor propels the remainder of the drop. Momentum conservation $m u=\rho R_{0}^{3} U$ yields

$$
U \sim \frac{E_{\mathrm{abs}}-E_{\mathrm{th}}}{\rho R_{0}^{3} \Delta H} u,
$$

that is, an increase in $U$ proportional to that in $E_{\text {abs }}$. Figure 4.5 (a) shows that this scaling argument, with a prefactor of 0.4 , is in good agreement with our experimental data.

With a description of the propulsion at hand, we now turn to the drop deformation. The expansion dynamics is directly affected by surface tension, which promotes the retraction and possibly the fragmentation of the drop. The key parameter describing the expansion is therefore the Weber number of the motion induced by the laser We $=\rho R_{0} U^{2} / \gamma$, which compares the drop-displacement kinetic energy to its surface energy. In our experiments $1 \leq \mathrm{We} \leq 60$. The impulsive acceleration of our drop from 0 to $U$ is similar to the impulsive stop of a drop impacting a solid with velocity $U$. We therefore use the momentum-based scaling for drop impact on solids $[42,44]$ to express the maximal radial expansion

$$
\frac{R_{\max }-R_{0}}{R_{0}} \sim \mathrm{We}^{1 / 2} \sim \sqrt{\frac{\rho R_{0} u^{2}}{\gamma}} \frac{E_{\mathrm{abs}}-E_{\mathrm{th}}}{\rho R_{0}^{3} \Delta H},
$$

in which the expression in terms of $E$ directly comes from Eq. (4.2). Expression (4.3), with a prefactor of 0.6 , is in good agreement with our experimental data up to $\mathrm{We} \sim 40$, when the drop starts fragmenting and the maximum expansion saturates (see Fig. 4.5 (b)). The scaling (4.3) has already been observed for drop impact onto solid substrates with negligible friction [42]. The present setup is however fundamentally different since, as mentioned above, the typical impact timescale, during which the drop accelerates, is decoupled from the inertial timescale: $\tau_{e} \ll \tau_{i}$.

\subsection{Numerical results}

To confirm that the interaction of the laser pulse with the drop can be modeled as a short recoil-pressure pulse exerted on the drop surface, we perform boundary-integral (BI) simulations [81-83]. We assume that the flow inside the drop is inviscid, irrotational, and incompressible, and solve the resulting Laplace equation for the flow potential. The method assumes axi-symmetry and therefore cannot be used to study the eventual fragmentation of the drop, but it does capture the initial phase of the drop deformation. 


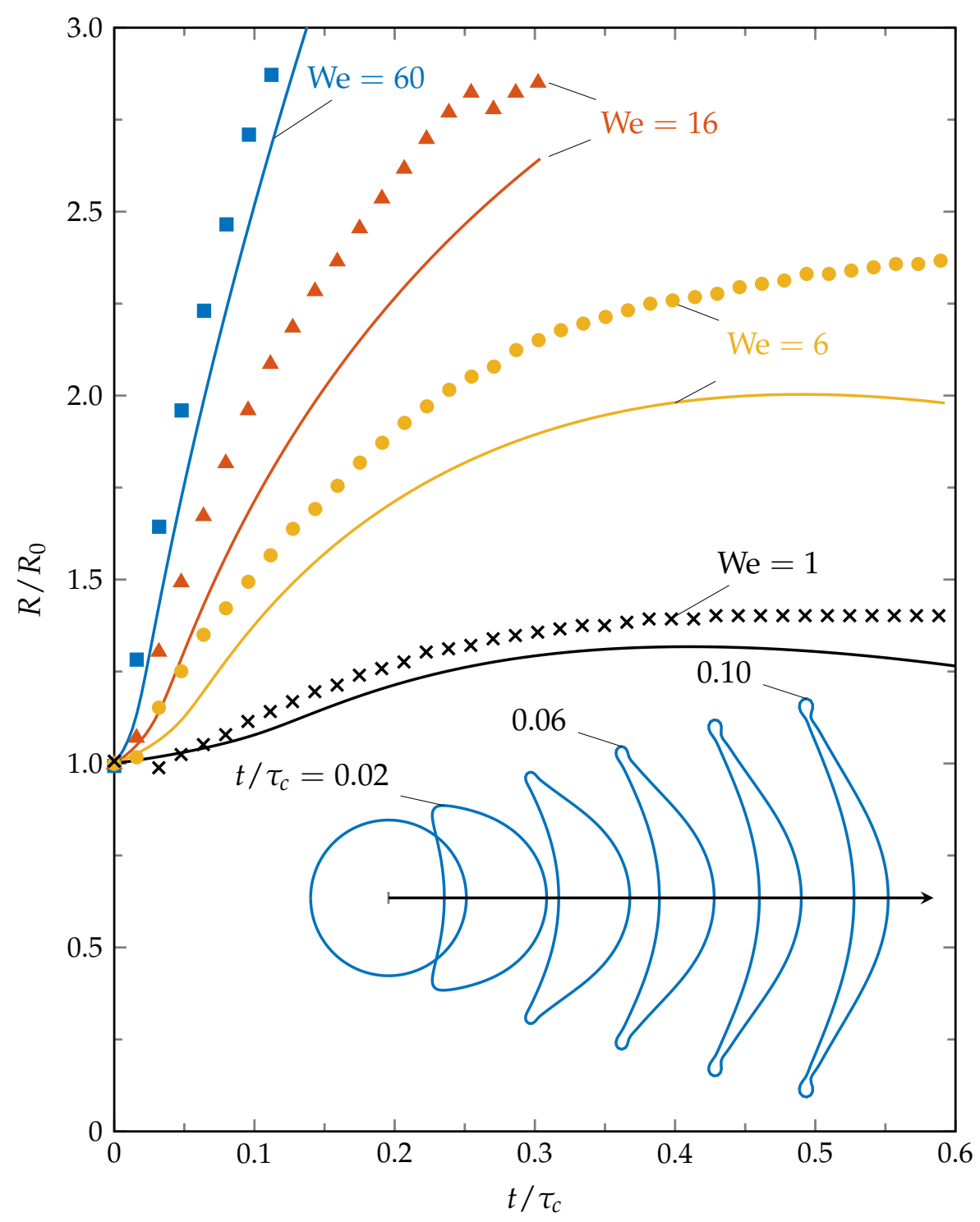

Figure 4.6: Radial expansion of the drop: experiments (markers) and BI simulations (solid lines). The corresponding image sequences are shown in Fig. 4.3. The inset shows the drop shape evolution from BI for $\mathrm{We}=60$ with an exaggerated center-ofmass displacement to separate the successive shapes. The simulations are stopped when the local sheet thickness becomes too thin to resolve the dynamics accurately. 
The laser pulse is modeled by applying a pressure boundary condition at the drop surface for a time duration $\tau_{e} \ll \tau_{i}$. We use a Gaussian pressure profile with a length scale based on the measured laser-beam profile and a pressure scale set to match the propulsion velocity observed in the experiment. This pressure scale is prescribed by the momentum conservation $p R_{0}^{2} \tau_{e} \sim$ $\rho R_{0}^{3} U$ (the prefactor can be obtained analytically, see $\S 6$ ). From Eq. (4.2) this recoil pressure can readily be expressed in terms of the absorbed energy.

The numerical drop shape evolution is shown in Fig. 4.6. It illustrates the added value of the simulations: not only the two-dimensional projection of the drop shape, but also the spatial and temporal evolution of the sheet thickness can be extracted, which is crucial when it comes to study fragmentation [42]. Moreover, Fig. 4.6 shows that the BI model quantitatively predicts the radial drop expansion observed for different Weber numbers. This confirms that a pressure pulse applied at the drop surface for a time much shorter than the hydrodynamic timescales $\left(\tau_{i}\right.$ and $\left.\tau_{c}\right)$ is indeed sufficient to describe the hydrodynamic response of a drop to the impact of a laser pulse.

\subsection{Conclusions}

We have shown that an opaque free-falling drop hit by a laser pulse propels and expands until fragmentation occurs. In the present case the laser energy is absorbed in a superficial layer of the drop such that the deposited energy per unit mass $E_{\text {abs }} / \rho R_{0}^{2} \delta \sim 0.1$ to $1 \mathrm{MJ} / \mathrm{kg}$ is comparable to the specific latent heat of vaporization. As a consequence, drop motion is induced by the recoil due to vaporization on the face of the drop that is hit by the laser. This results in a propulsion speed and a maximal radius of expansion that are both proportional to the pulse energy. The expansion dynamics is limited by surface tension and is similar to that of a drop impacting a solid, although with a laser pulse momentum transfer takes place on a much shorter timescale. Laserinduced drop fragmentation and the influence of the beam focusing require detailed studies and are left for $\S 6$ and $\S 7$. All results reported here should transpose directly to the shaping of liquid tin drops in EUV light sources. In a regime where a plasma is generated the propulsion mechanism may change, however, the Weber number remains the key parameter governing the hydrodynamic response. 


\section{A Measurement of energy absorption}

We first measure the laser-beam profile at the drop location (Fig. 4.7). To avoid saturation of the beam profiler we attenuate the laser energy by a factor of $10^{6}$ with two wedged windows and a reflective neutral-density filter. To determine the fraction of the light that is refracted into the drop at the air-liquid interface we use ray tracing (valid for $R_{0} \gg \lambda_{\mathrm{L}}=532 \mathrm{~nm}$ ). Since the dye is highly absorbent $\left(\delta \ll R_{0}\right)$, we neglect any light transmission through the drop. The initial condition of the ray tracing is set by the focal length of the focusing lens and the position of the drop in the laser beam (see Fig. 4.2). The distribution of rays in space and the energy attributed to each ray is chosen such that the set of rays resembles the measured beam profile. As a result, we estimate that in our experiments $20 \%$ of the laser-pulse energy is absorbed by the drop.

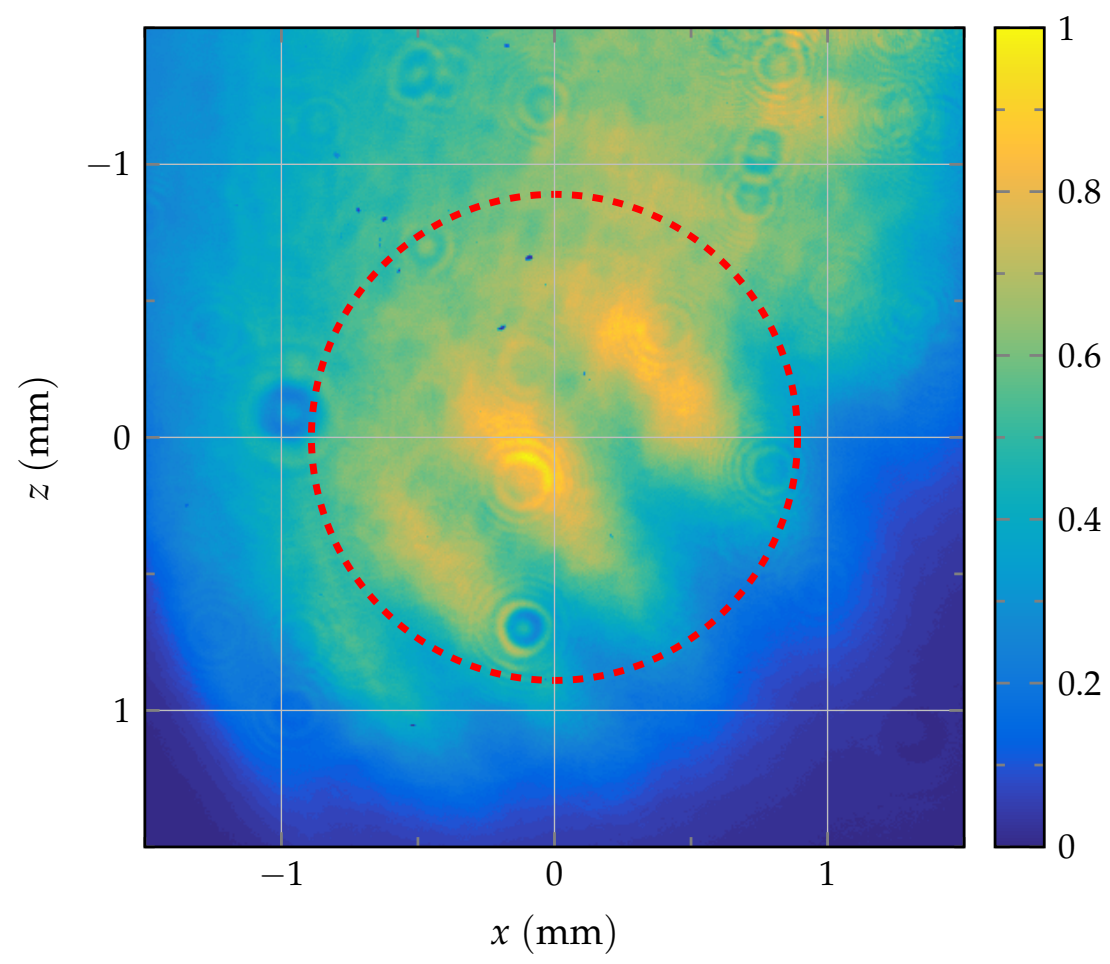

Figure 4.7: Normalized fluence of the laser beam measured at the drop location by a CCD beam profiler (BC106-VIS by Thorlabs). The profile depicted is the mean result of 10 single measurements. The dotted line indicates the position of the drop during the laser-impact experiments. 


\section{B Measurement of drop displacement}

For each recorded image we determine the center-of-mass position assuming rotational symmetry of the two-dimensional drop shape. Thereby, we ignore the concavity of the drop shape, which introduces an error in the determination of the center-of-mass position. This causes an apparent acceleration on the timescale $\tau_{i}$ in $Z$ (visible in Fig. 4.4).

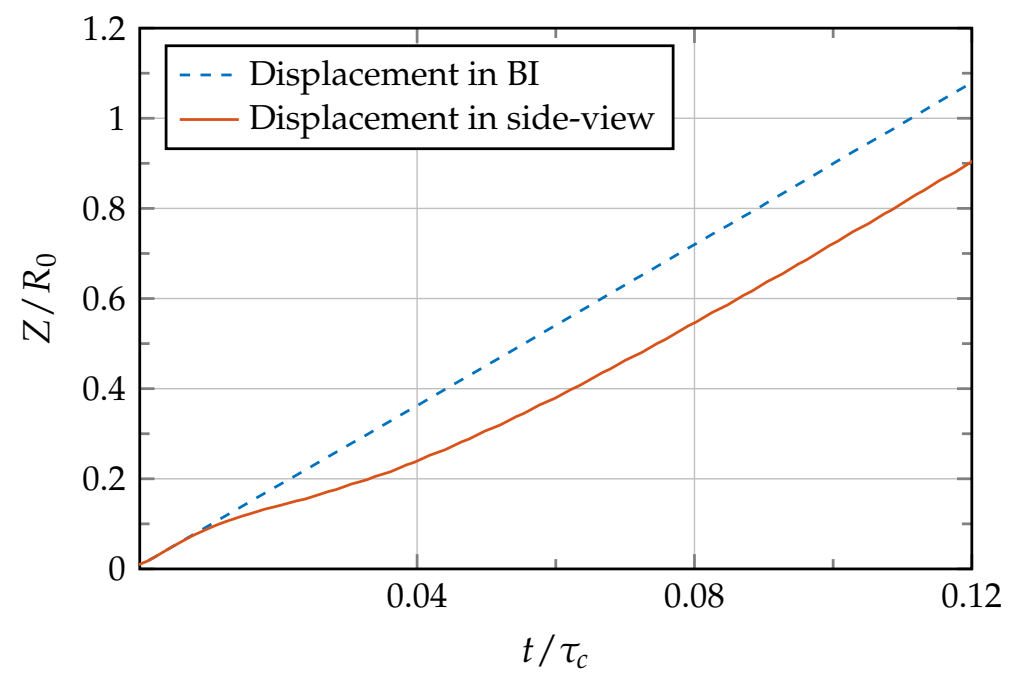

Figure 4.8: Center-of-mass displacement $Z$ of the drop (along $\vec{e}_{z}$, see Fig. 4.2) as a function of time in the BI simulation for $E_{\mathrm{abs}}=24 \mathrm{~mJ}$. The blue dashed line represents the actual center-of-mass displacement, whereas the red solid line is the center-ofmass displacement based on the two-dimensional projection of the simulated drop shape, which shows an artificial deceleration.

To confirm that this acceleration is an artificial result of our algorithm, we use the results from the BI simulations. In BI the full three-dimensional axisymmetric shape of the drop is known, and hence the exact center-ofmass displacement can be determined (dashed, blue line in Fig. 4.8). In addition, we compute the center-of-mass displacement based on the twodimensional projection of the BI data using the same procedure as we apply to the experimental side-view images (solid, red line in Fig. 4.8). The centerof-mass determined from the two-dimensional projection shows an artificial deceleration as soon as the drop shape becomes concave. However, Fig. 4.8 also shows that the final speed of the deforming drop is not affected by the apparent initial deceleration. Therefore, the error introduced by considering the projected drop shape only affects the initial deceleration of the center-ofmass, not its final speed. 


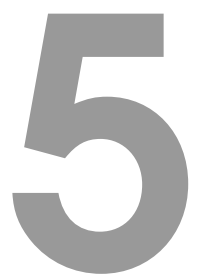

\section{Plasma propulsion of metallic micrometer-sized drops ${ }^{*,+}$}

The propulsion of a liquid indium-tin micrometer-sized drop by a nanosecond laser pulse is experimentally investigated. We capture the physics of the drop propulsion in a scaling law that accurately describes the plasma-imparted momentum transfer over nearly three decades of pulse energy, enabling the optimization of the laser-drop coupling. The subsequent drop deformation is described by an analytical model that accounts for the drop's propulsion velocity and the liquid properties. Comparing our findings to those from vapor-accelerated millimeter-sized water drops, we demonstrate that the fluid-dynamic response of laser-impacted drops is scalable and decoupled from the propulsion mechanism. By contrast, the physics behind the propulsion of metal drops differs from that of water. It is studied here in detail and under industrially relevant conditions as found in next-generation nanolithography machines.

\subsection{Introduction}

Micro-drops of tin can be used to create extreme ultraviolet light (EUV), e.g. in next-generation nano-lithography machines [11, 15], where the drops serve

${ }^{*}$ Published as: Dmitry Kurilovich, Alexander L. Klein, Francesco Torretti, Adam Lassise, Ronnie Hoekstra, Wim Ubachs, Hanneke Gelderblom, and Oscar O. Versolato. Plasma Propulsion of a Metallic Microdroplet and its Deformation upon Laser Impact. Physical Review Applied, 6(1):014018, 2016.

${ }^{\dagger}$ Dmitry Kurilovich and Alexander L. Klein contributed equally to this chapter. 
as mass-limited targets $[16,18]$ for a laser produced plasma (LPP). In such machines, these drops, several $10 \mu \mathrm{m}$ in diameter, are targeted by a focused nanosecond laser pulse at intensities that lead to optical breakdown and the creation of a high-density and high-temperature plasma [15, 18, 19]. Line emission from electron-impact-excited highly-charged tin ions in the plasma provides the EUV light $[15,18]$. For certain applications, it is beneficial to apply a dual-pulse sequence in which a laser "prepulse" is used to carefully shape the drop into a thin sheet that is considered advantageous for EUV production with the subsequent, much more energetic, "main pulse" [15-17].

Maximizing the conversion efficiency of such EUV sources, while minimizing the amount of fast ionic and neutral debris that limit EUV optics lifetime (see Ref. $[15,17,84]$ and references therein) requires a careful control over the drop propulsion and its shape. This in turn requires a profound understanding of the coupling of the laser to the drop as well as the drop's fluid-dynamic response to such a laser-pulse impact. While the propulsion and deformation of water drops due to laser-pulse impact has been studied in detail in $\S 4$, the interaction between liquid metal drops and a pulsed laser has remained unexplored. This interaction is governed by plasma dynamics with its distinct scaling laws [85-87].

Here, we present an analysis of the response of liquid indium-tin drops to a laser-pulse impact in a setting very close to the one used in the generation of EUV light for industrial application. We show that the drop response to a laser impact is governed by two physical processes that occur at completely different, and therewith separable, timescales. First, the interaction of the nanosecond laser pulse with the metal generates a plasma $[86,88]$ and induces propulsion of the drop. Second, fluid dynamics governs the shape-evolution of the drop, which takes place on a microsecond timescale. We reveal in detail the mechanism behind metal-drop propulsion by laser impact and present a scaling law for the propulsion speed as a function of laser energy that captures the onset of plasma formation. This scaling law enables us to optimize the laser-drop coupling for EUV generation purposes. Next, we discuss the similarities between metal- and water-drop propulsion by laser impact. We demonstrate that even though the metal-drop propulsion mechanism is completely different from that of water drops, the fluid-dynamic response is identical and well described by a universal analytical model.

\subsection{Experimental methods}

Figure 5.1 shows a detailed description of the experimental setup. We use an eutectic indium-tin alloy (50In-50Sn of $99.9 \%$ purity with liquid density 


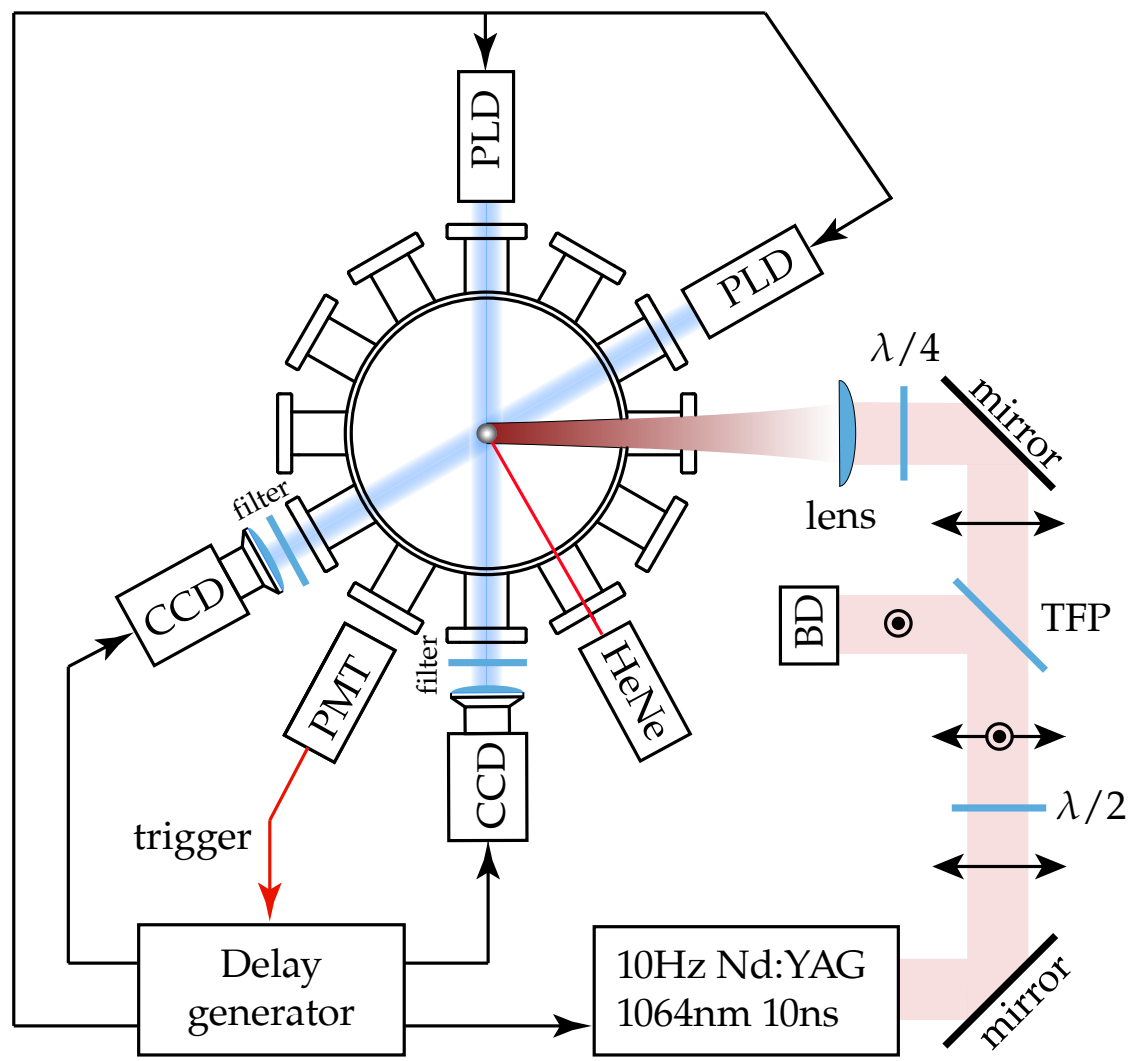

Figure 5.1: Sketch of the experimental setup in top view. Depicted is a vacuum vessel, typically at $10^{-7}$ mbar, with its many optical access ports. Drops are dispensed from a nozzle (not shown) held at a temperature of $250^{\circ} \mathrm{C}$. A horizontal light sheet produced by a helium-neon (HeNe) laser enables the triggering of the experiment: the light scattered by the falling drop is detected by a photomultiplier tube (PMT) that sends a signal to a delay generator. This delay generator in turn triggers an injection-seeded Nd:YAG laser that emits a pulse at $\lambda_{\mathrm{L}}=1064 \mathrm{~nm}$ with a duration of $\tau_{p} \approx 10 \mathrm{~ns}$ full width at half maximum (FWHM). A 1000-mm (500-mm) focallength N-BK7 lens produces a circular Gaussian focus of size approximately $115 \mu \mathrm{m}$ $(50 \mu \mathrm{m}) \mathrm{FWHM}$ at the position of the drop. The circle and arrow symbols along the optical axis indicate the direction of polarization of the laser beam. The energy of the laser pulse is controllable through the use of a half-wave plate $(\lambda / 2)$, a thin film polarizer (TFP), and a beam dump (BD). Before the focusing lens, a quarter-wave plate $(\lambda / 4)$ produces a circular polarization. The delay generator also triggers the pulsed laser diodes (PLD, at $850 \mathrm{~nm}$ wavelength; approximately 15-ns pulse length) one of which is aligned orthogonally to the Nd:YAG laser light propagation direction. The other PLD is angled at $30^{\circ}$ to it. In both cases, the PLD light passes through a band pass filter before it falls onto the CCD chip of a camera through a long-distance microscope and produces a shadowgraph. The magnifications are $2.0(2) \mu \mathrm{m} /$ pixel and $2.8(3) \mu \mathrm{m} /$ pixel for the orthogonal and $30^{\circ}$ shadowgraphs, respectively. 
$\rho=6920 \mathrm{~kg} / \mathrm{m}^{3}$ at $250^{\circ} \mathrm{C}$ [89]), a substance almost equivalent to pure tin in terms of atomic mass, density, and surface tension, but with a conveniently low melting point. Drops are dispensed from a drop generator at approximately $10-\mathrm{kHz}$ repetition rate, with final radii $R_{0} \approx 25 \mu \mathrm{m}$, and are falling down at a speed of approximately $12 \mathrm{~m} / \mathrm{s}$ in a vacuum vessel. No significant acceleration under gravity occurs on the timescale relevant for the experiment. The drops relax to a spherical shape before they pass through a horizontal light sheet produced by a helium-neon laser. The light scattered by the drops is used to trigger an injection-seeded $\mathrm{Nd}$ :YAG laser operating at $10-\mathrm{Hz}$ repetition rate that emits a pulse at its fundamental wavelength of $\lambda_{\mathrm{L}}=1064 \mathrm{~nm}$ with a duration of $\tau_{p} \approx 10 \mathrm{~ns}$ full width at half maximum (FWHM), focused down to a circular Gaussian spot. We study the propulsion for two different focusing conditions: approximately $115 \mu \mathrm{m}$ and $50 \mu \mathrm{m}$ FWHM at the position of the drop. In the first and main case of $115 \mu \mathrm{m}$ FHWM the width is larger than the drop diameter in order to decrease the sensitivity to laser-to-drop alignment and to provide a pressure profile required for obtaining a flat thin sheet, see $\S 6$. The finite geometrical overlap thus obtained precludes the full laser-pulse energy from reaching the target. The energy of the laser pulse is varied in a manner that does not affect the transversal mode profile of the laser beam.

Imaging of the drop is obtained by employing two pulsed laser diodes (PLDs). One of these is aligned orthogonally to the Nd:YAG laser light propagation direction to provide side-view images. The other PLD is angled at $30^{\circ}$ to it (see Fig. 5.1) for a (tilted) front-view. In both cases, the PLD light passes through a band pass filter before it falls onto the CCD chip of a camera through a long-distance microscope. The shadowgraphic images thus obtained are used to track size, shape, and velocity of the drop expansion in the direction along the Nd:YAG laser impact and perpendicular to the falling drop. A stroboscopic time series of different drops at various time delays (see Fig. 5.2) with an arbitrary number of frames is constructed by triggering once per Nd:YAG shot, each time with an increasing delay.

\subsection{Results}

The response of the In-Sn drop to laser impact is shown in Fig. 5.2. The laser pulse generates a plasma that expands away from the drop surface. As a result, the drop is accelerated to a speed $U \sim 0.5-350 \mathrm{~m} / \mathrm{s}$ on a time $\tau_{e}$ given by the lifetime of the generated plasma, which is known to be smaller than a few $\tau_{p}$ [90]. The subsequent deformation of the drop occurs on the much longer inertial timescale $\tau_{i}=R_{0} / U \approx 1 \mu$ s (here, $U$ is taken from the case shown in Fig. 5.2 (a)). The deformation is eventually slowed down by surface tension 
on the capillary timescale $\tau_{c}=\left(\rho R_{0}^{3} / \gamma\right)^{1 / 2} \approx 14 \mu \mathrm{s}$. The timescales relevant to this problem can thus be ordered (compare $\S 4$ )

$$
\tau_{p}<\tau_{e} \ll \tau_{i}<\tau_{c}
$$

which illustrates the clear separation of timescales between plasma generation as a cause for the propulsion on the one side and the fluid-dynamic response on the other side. Below, we discuss first the drop propulsion mechanism and second the drop deformation.

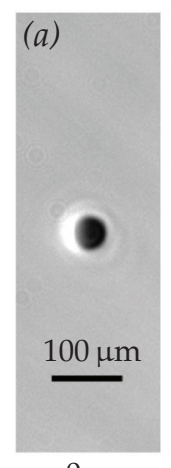

$0 \mu \mathrm{s}$

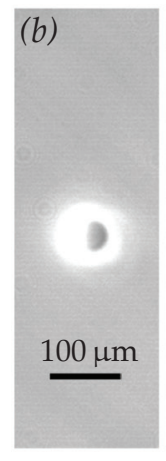

$0 \mu \mathrm{s}$

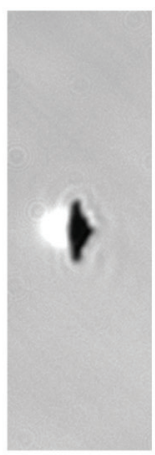

$0.4 \mu \mathrm{s}$

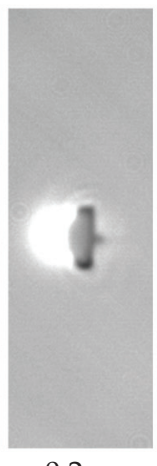

$0.2 \mu \mathrm{s}$

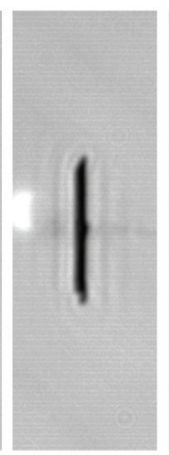

$2 \mu \mathrm{s}$

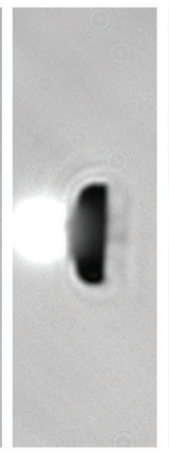

$0.4 \mu \mathrm{s}$

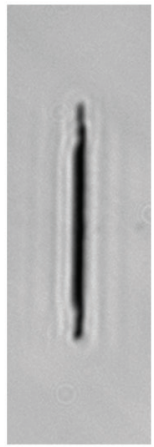

$4.5 \mu \mathrm{s}$

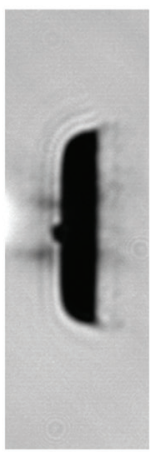

$1 \mu \mathrm{s}$

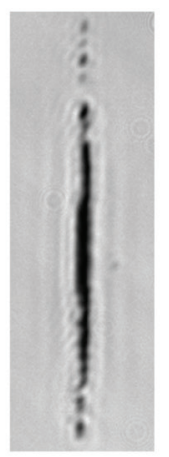

$9 \mu \mathrm{s}$

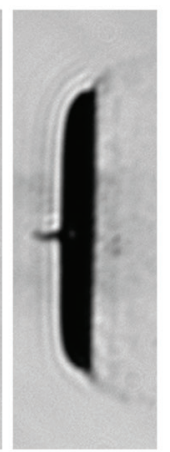

$1.7 \mu \mathrm{s}$

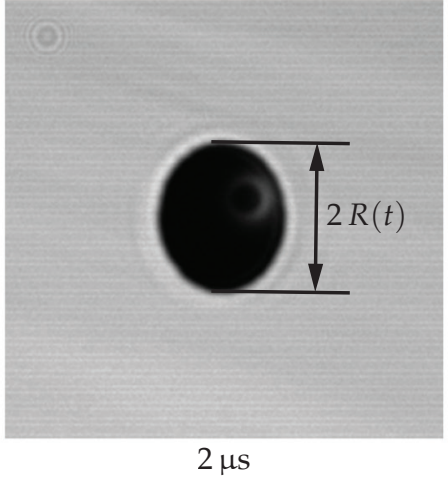

$2 \mu \mathrm{s}$

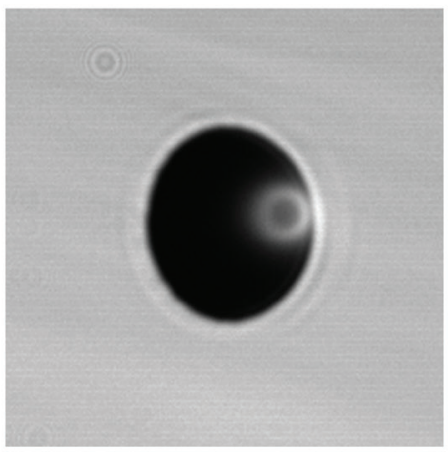

$1 \mu \mathrm{s}$

Figure 5.2: Shadowgraph images of In-Sn drops in side- and (tilted) front-view (see Fig. 5.1). (a) Expansion of an In-Sn drop as viewed from the side. Drops are irradiated with an approximately $115-\mu \mathrm{m}$ FWHM focused 10-ns Nd:YAG laser pulse impinging from the left with a total pulse energy of $7 \mathrm{~mJ}$. The stroboscopic drop expansion sequence is constructed from shadowgraphs of different drops at different time delays. A $30^{\circ}$ view is provided on the right. (b) Same as (a) but for a $70 \mathrm{~mJ}$ energy pulse, which gives rise to a faster expansion (note the different timescales). The white glow visible to the left of the expanding drop is plasma light. Images have been vertically aligned to center on the expanding drop. 


\subsubsection{Drop propulsion}

Using the shadowgraph images of the type shown in Fig. 5.2 we study the propulsion of the drop as a function of the laser-pulse energy, which is varied between 0.4 and $160 \mathrm{~mJ}$. Figure 5.3 shows that over the nearly three decades of laser-pulse energy studied in this work, the velocity of the drop ranges from below $1 \mathrm{~m} / \mathrm{s}$ to above $300 \mathrm{~m} / \mathrm{s}$ and appears to follow a power-law type scaling with the laser-pulse energy impinging on the drop, $E_{\mathrm{OD}}$. It is a fraction of the total pulse energy $E_{\mathrm{L}}$ given by the geometrical overlap of laser focus and drop. The similarity of the data obtained for the two different focusing conditions shows that $E_{\mathrm{OD}}$ is indeed the relevant parameter describing the scaling of $U$ in the present study. Significant deviations from this simple parametrization are expected for foci much smaller than the drop, see $\S 6$. To explain the observed scaling, we first discuss the mechanism through which the laser interacts with the metal.

The interaction of a high-intensity laser pulse with the drop is governed by plasma dynamics ${ }^{\ddagger}$. As soon as a plasma is generated, inverse-bremsstrahlung absorption [85] strongly decreases the initially high reflectivity of the metallic surface down to negligible values. This facilitates the further ablation of the target material. Analogous to the work on water drops in $\S 4$, we relate the propulsion speed $U$ of the micro-drop (mass $M$ ) to the amount of ablated mass $m$ through momentum conservation

$$
M U=m v,
$$

where $v$ is the velocity of the ejected mass along the axis of propulsion. The spatial distribution of this ejected mass peaks in the direction back towards the laser [91]. In the present intensity regime, simulations predict $v$ to range from $5-15 \times 10^{3} \mathrm{~m} / \mathrm{s}$ [92] and to be a function of the laser intensity. Experimentally, $v \approx 8.5 \times 10^{3} \mathrm{~m} / \mathrm{s}$ at a laser intensity of $2 \times 10^{11} \mathrm{~W} / \mathrm{cm}^{2}[84]$ which we take as a first estimate of the value for $v$ in the following. The ablated mass $m$ can be obtained employing a semi-empirical mass ablation law [93, 94],

$$
m=A \pi R_{0}^{2}\left[\left(\frac{I}{I_{0}}\right)^{5 / 9}\left(\frac{\lambda_{0}}{\lambda_{\mathrm{L}}}\right)^{4 / 9} Z^{3 / 8}\right] \tau_{p},
$$

with laser pulse intensity $I$ and $I_{0}=10^{11} \mathrm{~W} \mathrm{~cm}^{-2}$, wavelength $\lambda_{\mathrm{L}}$ and $\lambda_{0}=$ $1 \mu \mathrm{m}$, and atomic number $Z=49-50$ (for indium-tin). The term in square

\footnotetext{
$\ddagger$ The typical impulse $p_{l}$ exerted on the drop in the limit of fully absorbed light scales as $p_{l} \sim E / c$, with $E$ the energy of the absorbed light and $c$ the speed of light. Even at the highest pulse energies assuming a maximum fraction of absorbable light of approximately 0.5 , given by the geometrical overlap of laser and drop in the case of the 50- $\mu \mathrm{m}$ FWHM focus, this impulse would yield a typical drop speed $U \approx 0.5 \mathrm{~m} / \mathrm{s}$ which is negligible with respect to the observed speeds.
} 

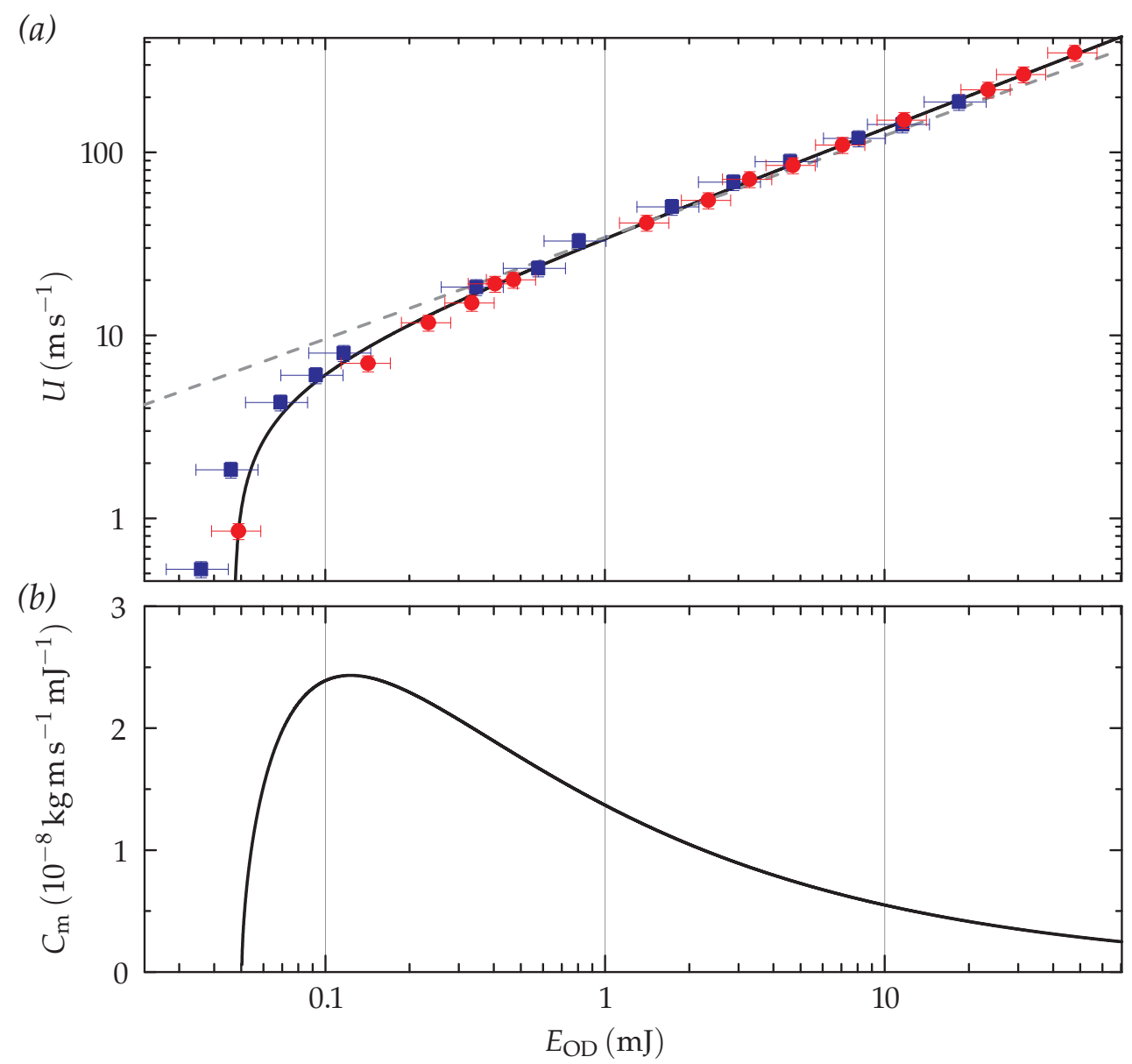

Figure 5.3: (a) Propulsion velocity $U$ of In-Sn drops as a function of total laser-pulse energy impinging on the drop $E_{\mathrm{OD}}$ for two different focusing conditions. Blue squares represent the data obtained at an approximately 115- $\mu \mathrm{m}$ FWHM focus; red circles represent the approximately $50-\mu \mathrm{m}$ case. Uncertainties (approximately $10 \% ; 1 \sigma$ ) for the velocity are smaller than the symbol size; $E_{\mathrm{OD}}$ is obtained with an approximately $25 \%(20 \%)$ uncertainty in case of an approximately $115-\mu \mathrm{m}(50-\mu \mathrm{m})$ FWHM focus. The gray dashed line represents a fit of Eq. (5.4) to the concatenated data excluding pulse energies below $0.2 \mathrm{~mJ}$. The black solid line depicts a fit of Eq. (5.7) to the concatenated data. (b) Momentum coupling coefficient $C_{\mathrm{m}}$ obtained from the fitted curve shown as a black solid line in (a). 
brackets is based on an analytical treatment of the one-dimensional expansion of a plasma from a plane surface [95]. The empirical constant $A$ was determined to be $3.0 \times 10^{3} \mathrm{~g} \mathrm{~cm}^{-2} \mathrm{~s}^{-1}$ for Sn for intensities ranging $10^{11}$ $10^{12} \mathrm{~W} \mathrm{~cm}^{-2}$ [94]. Using this value for $A$ in Eq. (5.3) we obtain an ablated mass fraction of $0.1 \%$ for the $7 \mathrm{~mJ}$ example shown in Fig. 5.2 (a). However, the input for $A$ as well as for $v$ above was obtained experimentally at laser intensities one to two orders of magnitude higher than are used in the current investigations. Therefore, we will leave their product as a free fit parameter in the following.

The model $(5.2,5.3)$ predicts a power-law dependence of the propulsion velocity on the laser intensity, which can be translated into a dependence on the total laser energy impinging on the drop $E_{\mathrm{OD}}$ for direct comparison to our measurements by virtue of the relation $I \sim E_{\mathrm{OD}}$, obtaining

$$
U=B E_{\mathrm{OD}}^{5 / 9} .
$$

The result of a single fit of Eq. (5.4) to both data sets excluding low pulse energies $E_{\mathrm{OD}}<0.2 \mathrm{~mJ}$, yields excellent agreement of this power-law with the data as is shown in Fig. 5.3. This agreement shows that $E_{\mathrm{OD}}$ is the correct parameter in scaling the data for the two focusing conditions. We obtain a proportionality constant $B=34(5) \mathrm{m} \mathrm{s}^{-1} \mathrm{~mJ}^{-5 / 9}$. Employing the empirical values from literature for $A$ and $v$ in Eq. $(5.2,5.3)$ we obtain a prediction of approximately $9 \mathrm{~m} \mathrm{~s}^{-1} \mathrm{~mJ}^{-5 / 9}$. A discrepancy was to be expected as the laser intensities in our experiments fall mostly outside the validated range [94] for Eq. (5.3) and for the estimate of $v$. Moreover, we disregarded the spherical geometry of the system. In light of these shortcomings, the agreement of the simple power law of Eq. (5.4) with both data sets is striking.

Power-law scaling of the momentum imparted by an expanding plasma has been extensively studied in the context of plasma thrusters as well as nuclear fusion (see e.g. Ref. [86, 88]). Empirically, from experiments on planar solid targets, it was found [86] that the ratio of the plasma pressure $p_{e}$ and laser intensity $I$ was excellently reproduced by the relation

$$
p_{e} / I \sim\left(I \lambda_{\mathrm{L}} \sqrt{\tau_{p}}\right)^{n},
$$

with exponent $n=-0.30$ (3) common to all studied materials [86, 88]. This scaling law was found to be valid over a very broad range of target and laser parameters, including the intensities and wavelengths relevant for EUV sources. Employing the scaling relation $U \sim I^{5 / 9}$ from Eq. (5.4) and linearizing $U \sim p_{e} \tau_{p}$, we obtain $n=-4 / 9 \approx-0.44$ from Eq. (5.5). On comparison with the value of $n$ valid for planar solid targets, we note that the difference could well reflect the change in target geometry from plane to spherical surfaces. 
The proof of the validity of this scaling law (Eq. (5.5)) for the current geometry with respect to $\lambda_{\mathrm{L}}$ and $\tau_{p}$ is left for future work.

At lower values of energy-on-drop $E_{\mathrm{OD}}$ in Fig. 5.3, below approximately $0.2 \mathrm{~mJ}$, the data no longer follow the power law. This is due to the physics governing the onset of the plasma formation upon the laser ablation of the metal. The laser fluence at the threshold of ablation is given by $[96,97]$

$$
F_{\mathrm{th}}=\rho \Delta H \sqrt{\kappa \tau_{p}} \approx 0.6 \mathrm{~J} \mathrm{~cm}^{-2},
$$

with latent heat of vaporization $\Delta H \approx 2.2 \mathrm{MJ} \mathrm{kg}^{-1}$ (taking the average of In and $\mathrm{Sn}$ in the mixture, both values being within $10 \%$ of this average), and thermal diffusivity $\kappa \approx 16.4 \mathrm{~mm}^{2} / \mathrm{s}$ [98]. At the onset, inverse-bremsstrahlung does not yet play a role and we have to take into account the reflectivity $\mathcal{R}$ of the surface $\$$, and multiply Eq. (5.6) with the factor $1 /(1-\mathcal{R})$ [101]. For simplicity we take $\mathcal{R}$ to be constant during the duration of the pulse. From these considerations, we obtain the prediction $F_{\mathrm{th}} \approx 5 \mathrm{~J} \mathrm{~cm}^{-2}$, identical to the approximately $5 \mathrm{~J} \mathrm{~cm}^{-2}$ from plasma modeling [92]. This threshold laser fluence translates into a minimum necessary pulse energy. Such a threshold energy can be straightforwardly included in our model by incorporating an offset pulse energy $E_{\mathrm{OD}, 0}$, such that the expression for the drop velocity reads

$$
U=\widetilde{B}\left(E_{\mathrm{OD}}-E_{\mathrm{OD}, 0}\right)^{\alpha} .
$$

Here, the power $\alpha$ is taken as a free parameter since generally $v=v(I)$ [92] and its scaling with intensity could influence the momentum scaling relation given by Eq. (5.4). A single fit of Eq. (5.7) to the full energy range of the concatenated data set (see Fig. $5.3(\mathrm{a})$ ) yields a power $\alpha=0.59(3)$, consistent with the postulated power of $5 / 9$. A proportionality constant $\widetilde{B}=35(5) \mathrm{m} \mathrm{s}^{-1} \mathrm{~mJ}^{-\alpha}$ is obtained similar to the result from the fit of Eq. (5.4) above. For the offset we find $E_{\mathrm{OD}, 0}=0.05(1) \mathrm{mJ}$, from which we in turn obtain $F_{\mathrm{th}}=2.4(8) \mathrm{J} \mathrm{cm}^{-2}$ by dividing the offset energy by $\pi R_{0}^{2}$. This value is in reasonable agreement with the simple estimate that yielded approximately $5 \mathrm{~J} \mathrm{~cm}^{-2}$. We conclude that all data are excellently described by a single fit of our model to the data, predicting the power-law scaling of $U$ with $E_{\mathrm{OD}}$ in Eq. (5.7) now including a threshold energy.

$\S$ The optical constants are, for pure tin in absence of laser light, $n=3.92, k=8.65$ (from [99] at $1.2 \mathrm{eV}$ at $523 \mathrm{~K}$ ) yielding a reflectivity of $\mathcal{R}=84 \%$. For indium a reflectivity of $\mathcal{R}=91 \%$ is obtained from $n=1.84, k=8.38$ (from [100] at $1.1 \mu \mathrm{m}$ at $295 \mathrm{~K}$ ). An estimate of the eutectic optical response is obtained from the averaging the reflectivities, yielding an absorption $1-\mathcal{R}=13 \%$. 


\subsubsection{Propulsion efficiency}

Having found an adequate description of the plasma propulsion mechanism over the complete measurement range, we can now derive an optimum condition for the laser-induced drop propulsion. The momentum coupling coefficient $C_{\mathrm{m}} \equiv p_{e} / I=(M U) / E$ [86] given by Eq. (5.5) is a figure of merit in plasma propulsion providing a measure for the propulsion efficiency in terms of total imparted momentum $M U$ per unit laser energy $E_{\mathrm{L}}$. Efficient propulsion implies an efficient momentum to kick the drop. This kick initiates the expansion of the drop into a thin sheet (see below). Propulsion and expansion speeds are coupled, and their ratio is a function of the focusing conditions as explained in $\S 6$. Industrial needs could dictate the minimum size of the focus, as it influences the laser-to-drop alignment stability. This will further influence the energy efficiency due to the geometrical overlap of laser focus and drop. For both focusing conditions, however, we can define optimum conditions under which a minimum amount of laser-pulse energy (on drop) is required to reach a given velocity. Less energy is then available to produce fast ionic and neutral debris that could limit machine lifetime [15]. Given the offset power law (Eq. (5.7)), $C_{\mathrm{m}}$ can be obtained as a function of pulse energy-on-drop $E_{\mathrm{OD}}$ (see Fig. $5.3(\mathrm{~b})$ ) reaching a maximum at $E_{\mathrm{OD}, \max }=E_{\mathrm{OD}, 0} /(1-\alpha)$ at which point a minimum amount of energy is used to achieve a given velocity. A sequence of optimal pulses, spaced just a few $\tau_{e}$ apart to allow the plasma to recombine, could then be used to achieve a specified velocity. Of course, the relation between the energy-on-drop $E_{\mathrm{OD}}$ and total laser-pulse energy $E_{\mathrm{L}}$ is given by the geometrical overlap of laser and drop. Our data (Fig. 5.3 (b)) thus indicate that the total energy efficiency is highest, at $E_{\mathrm{OD} \text {,max }}$, for the smallest spot size used in this work.

\subsubsection{Indium-tin-plasma vs water-vapor propulsion}

Our description of the plasma propulsion of In-Sn micro-drops shows striking analogies with recent work on water drops presented in $\S 4$. In that work, millimeter-sized water drops are dyed to efficiently absorb 532-nm light from a Nd:YAG laser pulse, at intensities well below the threshold for optical breakdown to avoid plasma generation. Instead, water vapor is expelled at its thermal speed and accelerates the drop. In the dyed-water experiments, laser light is absorbed in a thin layer of thickness $\delta$, given by the optical penetration depth. This layer first needs to be heated from room temperature $T_{0}$ to boiling point $T_{\mathrm{b}}$, yielding an expression for the threshold fluence $F_{\mathrm{th}, \mathrm{w}}=\rho_{\mathrm{w}} \delta c_{\mathrm{v}}\left(T_{\mathrm{b}}-\right.$ $T_{0}$ ), where $c_{\mathrm{V}}$ is the specific heat capacity of water and $\rho_{\mathrm{W}}$ is its density, see $\S 4$. Any additional energy leads to vaporization of the liquid, expelling mass at a 
thermal velocity $u \approx 400 \mathrm{~m} / \mathrm{s}$ which leads to the propulsion of the water drop. When comparing the onset for propulsion of water drops to the threshold fluence for In-Sn (Eq. (5.6)), one observes that for water, the mass affected by the laser is set by the optical penetration depth, whereas for In-Sn it is set by the thermal penetration depth $\sqrt{\mathcal{k} \tau_{p}}$. The energy density required for drop propulsion is given by the sensible heat for water, and by the latent heat $\Delta H$ for In-Sn. The In-Sn drop surface is shielded from the laser light by the plasma that develops at timescales well shorter than the pulse length. Laser light is absorbed by this plasma layer, heating it to high temperatures (several $10 \mathrm{eV}$ ). As a result, a relatively small amount of mass ablated is expelled at high velocities $v \gg u$, thus mass-efficiently propelling the drop. In conclusion, even though the physical mechanism behind the propulsion of In-Sn and water drops is different, both processes are described by the forceful directive expulsion of material at a given velocity from a thin layer facing the laser light.

As we will demonstrate below, the fluid-dynamic response to the laser impact described here and in $\S 4$ is completely independent from the exact mechanism of propulsion. Mapping the system to the dimensionless numbers governing fluid dynamics allows for an identical treatment of said response for indium-tin and water. The efficiency of the plasma propulsion process enables the study of the response of the metallic drop over a much larger parameter space than for water, including the industrially relevant large-Weber-number subspace [16], which previous experiments on water drops in $\S 4$ were unable to probe.

\subsubsection{Drop deformation}

The expansion of the drop for the $115-\mu \mathrm{m}$ focusing case is studied by changing the time delay between the pulse from the Nd:YAG laser and the shadowgraphy lasers in steps of $100 \mathrm{~ns}$. A stroboscopic movie of the deformation dynamics is thus obtained (see Fig. 5.2) with an adequate temporal resolution. We measure the expanded radius of the drop over time as shown in Fig. 5.4 (a) using the side-view shadowgraphs, in which the drop motion is captured within the depth of field of the imaging optics at all time delays. In addition, the front-view shadowgraphs are used to make sure the drop expands into an axisymmetric shape. For comparison, we also include data from millimeter-sized water drops, the deformation of which takes place on a time and length scale several orders of magnitude larger than for the In-Sn data (see inset in Fig. 5.4 (a)). However, by appropriately rescaling the data by the initial drop radius $R_{0}$ as the characteristic length scale and the capillary time $\tau_{c}=\left(\rho R_{0}^{3} / \gamma\right)^{1 / 2}$ as the characteristic timescale for the drop expansion and subsequent retraction, we can represent all data in one graph: Fig. 5.4 (b). 


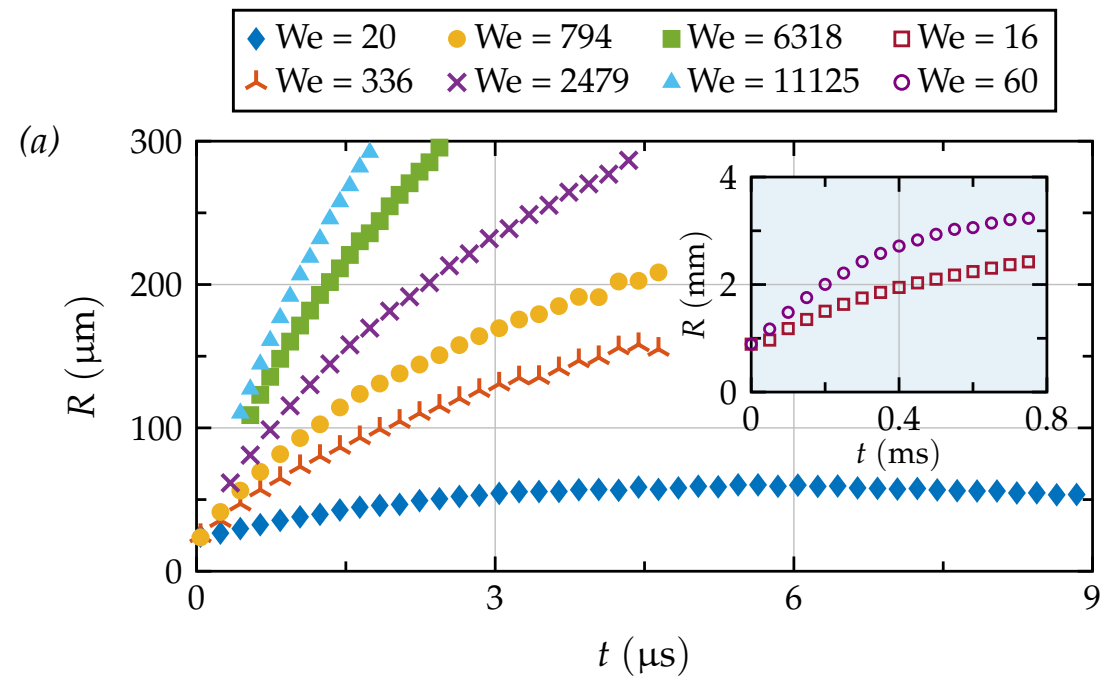

(b)
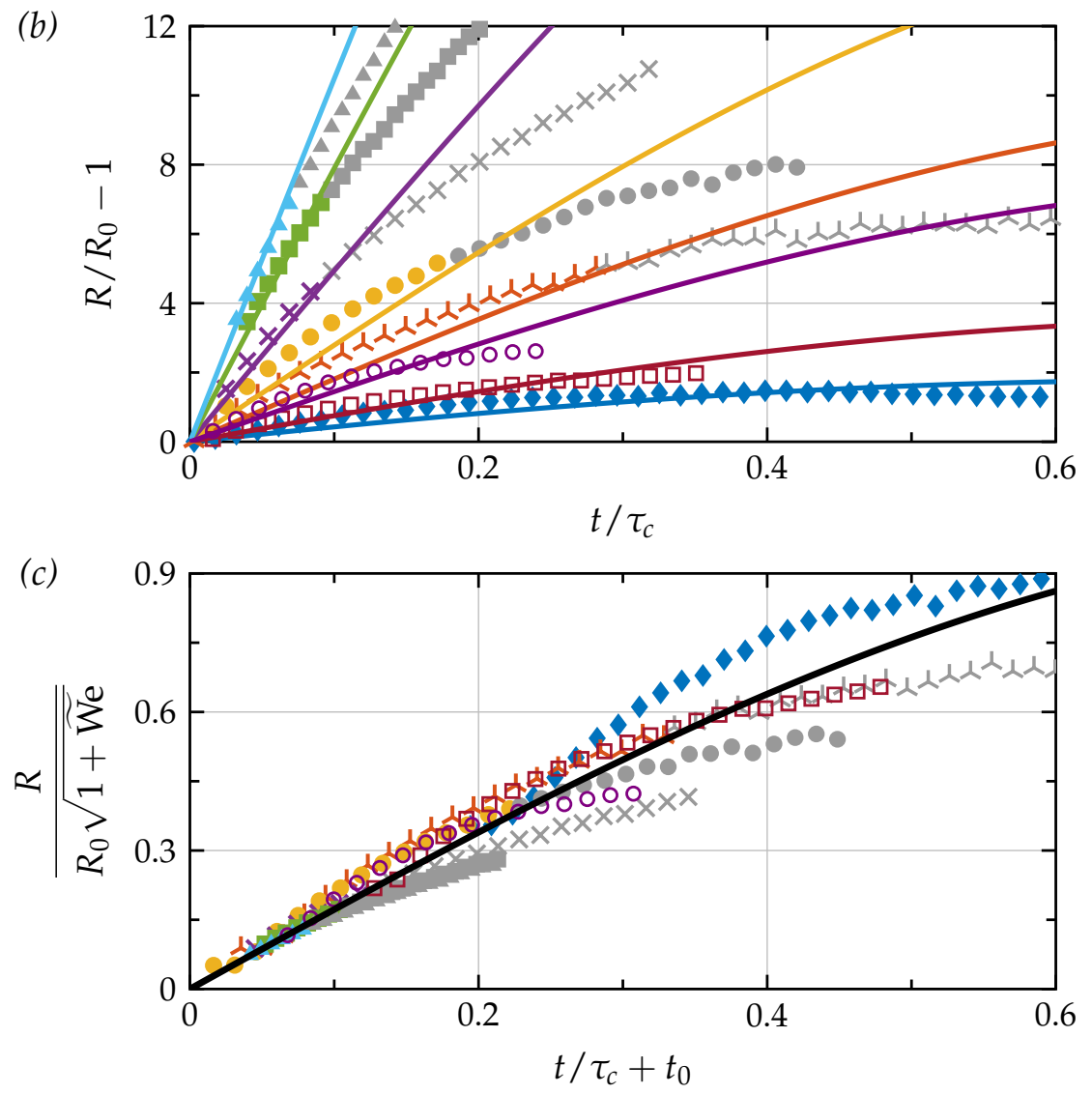

Figure 5.4: (Continued on the following page.) 
Figure 5.4: (Figure is found on the previous page.) Radial expansion of In-Sn and water drops as function of time. (a) Dimensional plot of the experimental data for In-Sn drops determined from shadowgraphy (see Fig. 5.2), with Weber numbers calculated using the drop velocities from Fig. 5.3. The inset shows data for millimeter-sized water drops taken from the previous study in $\S 4$ (hollow markers). (b) Experiments (markers) and theory (solid lines, Eq. (5.8)) in a dimensionless representation. The data points are depicted in gray from the time onward at which formation of ligaments becomes apparent and comparison with the present theory is no longer relevant. A comparison between model and water data taken from §6. (c) Experimental data (markers) and theory (solid line) collapsed to a universal curve using a scaled Weber number $\widetilde{W e}$ and time offset $t_{0}$ (see main text).

For indium-tin, $\tau_{c} \approx 14 \mu$ given its surface tension $\gamma=538 \mathrm{mN} / \mathrm{m}$ [89]. The expansion dynamics of the drop can be described in terms of the Weber number We $=\rho R_{0} U^{2} / \gamma$, a dimensionless number that is a measure for the relative importance of the drop's kinetic energy compared to its initial surface energy, see also $\S 4$ and $\S 6$.

In the limit where the drop expands into a flat, thin sheet that subsequently recedes due to surface tension an analytical expression can be found for the radius $R$ as function of time $t$ (for the derivation see $\S 6$ )

$$
\begin{aligned}
\frac{R(t)}{R_{0}}= & \cos \left(\sqrt{3} t / \tau_{c}\right)+ \\
& \left(\frac{2}{3}\right)^{1 / 2}\left(\frac{E_{\mathrm{k}, \mathrm{d}}}{E_{\mathrm{k}, \mathrm{cm}}}\right)^{1 / 2} \mathrm{We}^{1 / 2} \sin \left(\sqrt{3} t / \tau_{c}\right),
\end{aligned}
$$

where the parameter $E_{\mathrm{k}, \mathrm{d}} / E_{\mathrm{k}, \mathrm{cm}}$ is the partition of the total kinetic energy given to the drop by the laser impact into a deformation kinetic energy $E_{\mathrm{k}, \mathrm{d}}$ and propulsion kinetic energy of the drop's center-of-mass $E_{\mathrm{k}, \mathrm{cm}}$. This parameter depends on the laser beam profile and can be determined analytically as shown in $\S 6$. For all practical purposes in the present study, the laser beam profile can be considered flat, which gives $E_{\mathrm{k}, \mathrm{d}} / E_{\mathrm{k}, \mathrm{cm}}=0.5$ as shown in $\S 6$. The water data from $\S 4$ was obtained with a relatively more focused laser beam, for which $E_{\mathrm{k}, \mathrm{d}} / E_{\mathrm{k}, \mathrm{cm}}=1.8$ was calculated. Figure $5.4(\mathrm{~b})$ shows that the model prediction is in good agreement with the experimental data taking into account that it is derived from first principles and does not incorporate any fitting parameter. In the comparison we truncated the experimental data where the formation of ligaments becomes apparent in the front-view images, as at this point in time the assumption of a thin sheet of constant mass is clearly violated.

With the analytical expression at hand, the indium-tin and water data can 
be collapsed onto a universal curve by employing a trigonometric identity to rewrite Eq. (5.8) to

$$
\frac{R(t)}{R_{0} \sqrt{1+\widetilde{\mathrm{We}}}}=\sin \left(\sqrt{3}\left(t / \tau_{c}+t_{0}\right)\right),
$$

introducing an offset time $t_{0}$ and a scaled Weber number $\widetilde{W}$ that depends on the energy partition ratio $E_{\mathrm{k}, \mathrm{d}} / E_{\mathrm{k}, \mathrm{cm}}$,

$$
\begin{aligned}
\widetilde{\mathrm{We}} & =\frac{2}{3} \frac{E_{\mathrm{k}, \mathrm{d}}}{E_{\mathrm{k}, \mathrm{cm}}} \mathrm{We}, \\
t_{0} & =\frac{1}{\sqrt{3}} \tan ^{-1}\left(\widetilde{\mathrm{We}}^{-1 / 2}\right) .
\end{aligned}
$$

The data scaled with Eq. (5.9) indeed overlap completely and agree with the now single theoretical prediction (see Fig. 5.4 (c)). This collapse demonstrates that the expansion dynamics, given the above scaling relation, are successfully described by a single dimensionless parameter, which spans nearly three decades in our experiments including the conditions relevant for industry [16].

\subsection{Conclusions}

We demonstrated that a micrometer-sized free-falling In-Sn liquid drop hit by a high-intensity 10-ns-long laser pulse is propelled by a plasma pressure "kick". The propulsion dynamics can be well understood in terms of a power law that describes the dependence on the laser-pulse energy and incorporates an offset parameter to account for the threshold of plasma generation. This scaling law provides a useful tool to optimize the momentum coupling of the laser to the target. The description of the propulsion as a short recoil-pressure is similar to that presented in $\S 4$, where a millimeter-sized water drop is accelerated by the directional emission of vapor upon the absorption of light from a short laser pulse. We find remarkable one-to-one correspondences between the two propulsion mechanisms, including the description of an analogous onset effect, even though the physical origins of the propulsion are completely different. Continuing its flight, the drop expands until fragmentation occurs. By a proper rescaling we show that all indium-tin and water data can be collapsed onto a universal curve that is accurately described by a previously developed analytical model, here applied under industrially relevant conditions. The results and scaling laws presented in this work provide a tool to optimize and control the drop shaping by laser pulse impact for EUV lithography applications. 


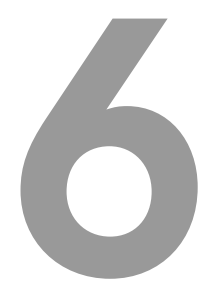

\title{
Drop deformation by laser-pulse impact $^{*}$
}

\begin{abstract}
A free-falling, absorbing liquid drop hit by a nanosecond laser-pulse experiences a strong recoil-pressure kick. As a consequence, the drop propels forward and deforms into a thin sheet which eventually fragments. We study how the drop deformation depends on the pulse shape and drop properties. We first derive the velocity field inside the drop on the timescale of the pressure pulse, when the drop is still spherical. This yields the kinetic-energy partition inside the drop, which precisely measures the deformation rate with respect to the propulsion rate, before surface tension comes into play. On the timescale where surface tension is important, the drop has evolved into a thin sheet. Its expansion dynamics is described with a slender-slope model, which uses the impulsive energy-partition as an initial condition. Completed with boundaryintegral simulations, this two-stage model explains the entire drop dynamics and its dependance on the pulse shape: for a given propulsion, a tightly focused pulse results in a thin curved sheet which maximizes the lateral expansion, while a uniform illumination yields a smaller expansion but a flat symmetric sheet, in good agreement with experimental observations.
\end{abstract}

*Published as: Hanneke Gelderblom, Henri Lhuissier, Alexander L. Klein, Wilco Bouwhuis, Detlef Lohse, Emmanuel Villermaux, and Jacco H. Snoeijer. Drop deformation by laser-pulse impact. Journal of Fluid Mechanics, 794:676-699, 2016. 


\subsection{Introduction}

A laser pulse interacting with an absorbing liquid body can deposit a finite amount of energy, concentrated both in time and space, which eventually triggers a dramatic hydrodynamic response. Focused nanosecond pulses have for instance been used to induce cavitation in liquids confined in capillary tubes [33-35], or jetting and spraying in sessile drops [36]. These situations involving a liquid close to a wall result in localized flows. By contrast, we consider here the situation of a mobile liquid body: the impact of a nanosecond laser pulse onto an absorbing unconfined liquid drop, which, as first described in $\S 4$, has a global hydrodynamic response to the pulse: the drop propels forward at a speed of several meters per second, strongly deforms and eventually fragments (see Fig. 6.1). This dynamics is similar to that following a mechanical impact such as on a solid substrate or a pillar, which has been studied thoroughly [see e.g. Ref. 36, 42, 44, 45, 47, 102, 103], including a few studies on the fragmentation of the drop $[42,49,79,80,104]$. A laser proves to be an adequate tool to vary the extension of the impact without affecting the initial drop geometry. However, how a drop deforms and fragments as a results of a laser impact are still a largely open questions.

An important application of drop deformation by laser-pulse impact is found in laser-produced plasma light-sources for extreme ultraviolet (EUV) nanolithography. In these sources a nanosecond laser pulse pre-shapes a
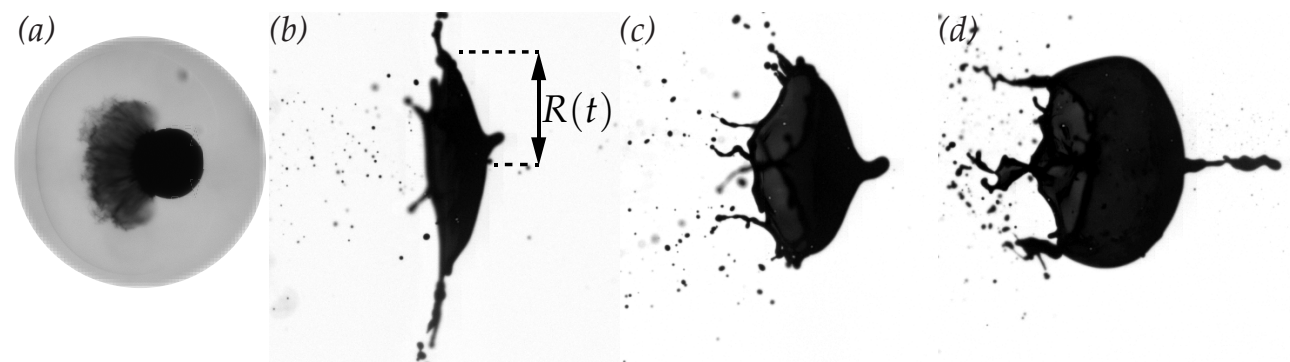

Figure 6.1: Response of a free-falling dyed water drop of initial radius $R_{0}=0.9 \mathrm{~mm}$ to a 10-ns laser pulse $\left(\lambda_{\mathrm{L}}=532 \mathrm{~nm}\right)$ impacting from the left. (a) $t=5 \mu \mathrm{s}\left(t / \tau_{c}=0.0016\right.$, with $\tau_{c}$ the capillary time) after the pulse, a shock wave has propagated in the air and a mist cloud has developed at the drop surface, but the drop itself has not yet moved. $(\mathrm{b}-\mathrm{d}) t=1.2 \mathrm{~ms}\left(t / \tau_{c}=0.39\right)$ after the pulse, the drop has propelled and deformed into a thin sheet (same magnification as in (a)), whose shape and lateral expansion $R(t)$ depend not only on the energy $E_{\mathrm{abs}}$ absorbed by the drop, but also on the width of the laser beam on the drop surface $\sim \sigma R_{0}$ (see $\S 6.3$ ): (b) uniform illumination of the drop $\left(\sigma \simeq 0.75, E_{\mathrm{abs}}=29 \mathrm{~mJ}\right.$, acquired Weber number We $\simeq 32 \pm 6$ ), (c) slightly focused laser ( $\sigma \simeq 0.48, E_{\mathrm{abs}}=20 \mathrm{~mJ}$, We $\simeq 28 \pm 8$ ), and (d) tightly focused laser $\left(\sigma \simeq 0.29, E_{\mathrm{abs}}=20 \mathrm{~mJ}, \mathrm{We} \simeq 72 \pm 29\right)$. 
falling liquid tin drop into a thin sheet, which is subsequently ionized by a second laser pulse $[15,17]$. To maximize the conversion of liquid tin to plasma, a precise control of the drop shape and stability that result from the first laser impact is crucial. That is, the dynamic response of a liquid drop to the impact of a laser pulse has to be resolved.

In $\S 4$ we focused on the question of how the laser transfers momentum to the liquid body. We showed that the key driving mechanism for the drop propulsion and deformation observed in experiments is the local and asymmetric boiling of the liquid induced by the absorption of the laser energy on the illuminated side of the drop. In a dyed (and hence absorbing) drop this absorption occurs in a thin, superficial layer of liquid, whose thickness $\delta \sim 10 \mu \mathrm{m}$ is set by the penetration depth of the laser light. As a result this layer boils and a shock wave is emitted in the surrounding air, followed by the directive emission of vapor and mist (see Fig. 6.1 (a)). This expelled mass is only a tiny fraction of the total mass of the drop, $\delta / R_{0} \sim 10^{-2}$, with $R_{0}$ the initial drop radius. The resulting vapor-recoil pressure on the drop surface both deforms the drop and propels it forward (Fig. 6.1 (b-d)) at a velocity

$$
U \sim \frac{E_{\mathrm{abs}}-E_{\mathrm{th}}}{\rho R_{0}^{3} \Delta H} u .
$$

This propulsion velocity scales linearly with the absorbed laser energy $E_{\text {abs }}$ beyond the threshold energy $E_{\text {th }}$ needed to heat the liquid layer to the boiling point, where $\rho$ is the liquid density, $\Delta H$ the latent heat of vaporization and $u$ the thermal speed of the expelled vapor. The drop propulsion is accompanied by a lateral expansion that scales as

$$
\frac{R_{\max }-R_{0}}{R_{0}} \sim \mathrm{We}^{1 / 2},
$$

where the Weber number is defined as We $=\rho R_{0} U^{2} / \gamma$, and $\gamma$ is the liquid surface tension. Hence, both the propulsion speed and the maximal radius of expansion are proportional to the laser-pulse energy (beyond the threshold). However, not only the energy of the laser pulse, but also the pulse shape and focus have a strong influence on the drop deformation and propulsion, as Fig. $6.1(\mathrm{~b}-\mathrm{d})$ shows. Although the absorbed laser energy is similar in the three cases shown, the resulting drop shapes differ completely: an unfocused laser beam deforms the drop into an almost flat sheet, whereas a focused beam gives rise to a strongly curved, bag-like drop shape.

Before seeking for understanding these differences, it is worth remembering the clear separation of the timescales involved in the problem described in $\S 4$, which we illustrate in Fig. 6.2. The impact of a few milli-Joule laser pulse 


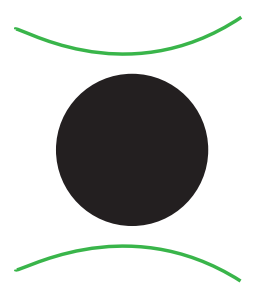

$\tau_{p}=10^{-8} \mathrm{~s}$

$\tau_{e}=10^{-5} \mathrm{~s}$

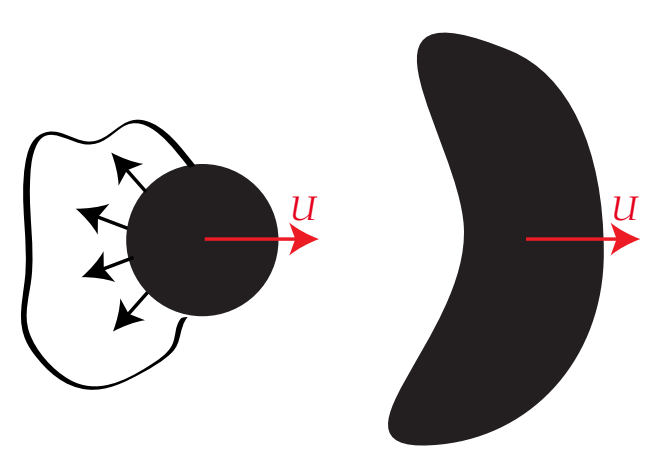

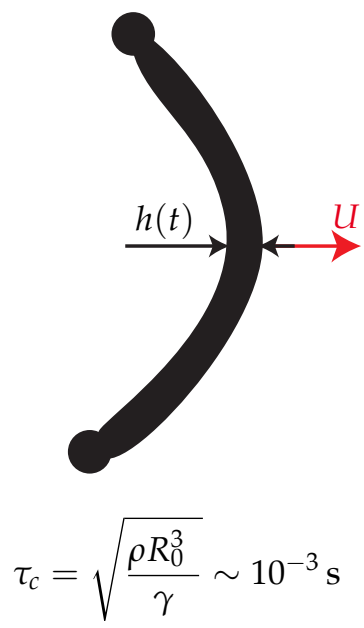

Figure 6.2: Illustration of the timescale separation in the problem. The laser interacts with the drop on time $\tau_{p}$, the drop reaches its center-of-mass velocity $U$ on the vapor expulsion time $\tau_{e}$. The drop subsequently deforms on the inertial time $\tau_{i}$ into a thin sheet with time-dependent thickness $h(t)$, which undergoes a surface-tension limited expansion on the capillary time $\tau_{c}$.

with a duration $\tau_{p} \sim 10^{-8}$ s onto a liquid drop results in the directive ejection of vapor and mist by the drop on a timescale $\tau_{e} \sim 10^{-5} \mathrm{~s}$. The vapor recoil then gives rise to the drop propulsion and deformation. Therefore, the laser-drop interaction can successfully be modeled as a recoil-pressure pulse exerted on the drop surface for a duration $\tau_{e}$, see $\S 4$. It is clear from Fig. 6.1 (a), that on this timescale the drop does not deform: both the laser pulse duration $\tau_{p}$ and the vapor-recoil duration $\tau_{e}$ are much shorter than the inertial and capillary timescales, respectively $\tau_{i}=R_{0} / U \sim 10^{-4} \mathrm{~s}$ and $\tau_{c}=\left(\rho R_{0}^{3} / \gamma\right)^{1 / 2} \sim 10^{-3} \mathrm{~s}$, on which the drop propels, deforms and fragments (Fig. 6.1 (b-d)). To estimate the inertial timescale we used a characteristic velocity of $U \sim 1 \mathrm{~m} / \mathrm{s}$, based on our experimental observations.

This chapter of the thesis aims to elucidate how the laser-pulse shape and focus affect the drop deformation and propulsion. To this end, we employ both analytical and numerical modeling and make use of the separation of the timescales $\tau_{p} \ll \tau_{e} \ll \tau_{i}<\tau_{c}$. In $\S 6.2$ and $\S 6.3$ we follow a pressure impulse approach as described by Batchelor [105, § 6.10], Cooker and Peregrine [106], and Antkowiak et al. [107] to obtain, for an arbitrary pulse shape, the velocity field in the drop and the kinetic-energy partition between the deformation and the translation of the drop on the timescale $\tau_{e}$, i.e. the initial lateral expansion rate of the drop relative to its propulsion speed. Surprisingly, we find that the maximum expansion rate is achieved when one focuses the laser pulse 
into a tight spot, whereas a flat (symmetric) expanding drop is obtained only with a uniform laser-beam profile. On the intermediate timescale $\tau_{i}$ the drop deforms significantly and a purely ballistic approach is no longer applicable. We use in $\S 6.3$ a numerical boundary-integral (BI) method [81-83, 108-111] to confirm the main features of the deformation and the precise detail of the flow. For an unfocused laser pulse (Fig. 6.1 (b)) the drop evolves into a flat, thin sheet. In $\S 6.4$ we use the kinetic-energy partition obtained from the early-time analytical model and follow the method of Villermaux and Bossa [80] to describe the surface-tension limited expansion of this sheet on the late timescale $\tau_{c}$.

\subsection{Problem formulation \& methods}

We consider the response of a liquid drop to a pressure pulse, i.e. a pressure field with magnitude $p_{e}$ applied at the interface on one side of the drop for a duration $\tau_{e}$. The absolute impulse scale $p_{e} \tau_{e}$ sets the propulsion velocity of the drop through momentum conservation (see Eq. (6.4) below). As we discussed above, this velocity is in turn directly related to the laser-pulse energy through Eq. (6.1). The problem thus amounts to determining the shape and the rate of deformation of the drop. In $\S 6.2 .1$ we introduce an analytical model for the early-time dynamics of the drop $\left(t \sim \tau_{e}\right)$. To complement the analytical model and describe the dynamics at later times $\left(t \sim \tau_{i}, \tau_{c}\right)$ we use the BI model that is discussed in $\S 6.2 .2$.

\subsubsection{Early-time dynamics: analytical model}

We characterize the ratio between the inertial timescale on which the drop deforms and the vapor-expulsion time on which the drop acquires it center-ofmass speed by the impact number

$$
I=\frac{\tau_{i}}{\tau_{e}}=\frac{R_{0}}{U \tau_{e}} .
$$

Note that since $I \gg 1$ the drop does not deform on the timescale of the pressure pulse, as is shown in Fig. 6.1 (a). To find the post-impact velocity field we therefore naturally consider the impulsive response of a spherical drop. Figure 6.3 shows a sketch of the problem geometry and indicates both the spherical $(r, \theta, \phi)$ and cartesian coordinates $(x, y, z)$. Both the initial configuration and the pressure pulse are symmetric around the laser axis (z-axis), and we therefore seek a velocity field that is also symmetric. The pressure pulse applied on the drop surface sets the fluid in motion inside the 


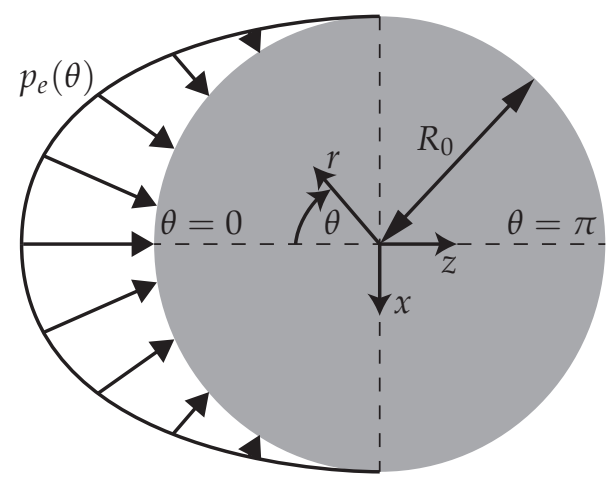

Figure 6.3: Sketch of the problem. The axisymmetric pressure pulse $p_{e}(\theta)$ applied on the surface of a drop of radius $R_{0}$. The spherical $(r, \theta, \phi)$ and cartesian $(x, y, z)$ coordinate systems are indicated.

entire drop. The axial propulsion speed $U$ of the drop (see Fig. 6.3), i.e. its center-of-mass velocity, follows from the global momentum conservation

$$
\int_{0}^{\tau_{e}} \int_{A} p_{e} \mathbf{e}_{\mathbf{z}} \cdot \mathrm{d} \mathbf{A} \mathrm{d} t=\frac{4}{3} \pi \rho R_{0}^{3} U,
$$

with $A$ the surface of the drop.

To describe the flow field inside the drop we follow the same approach as Batchelor [105, § 6.10], Cooker and Peregrine [106], and Antkowiak et al. [107]. The pressure field establishes on the sonic timescale $R_{0} / c \sim 10^{-6} \mathrm{~s}$, with $c$ the speed of sound in the liquid, which is much shorter than the pressure pulse duration $\tau_{e} \sim 10^{-5} \mathrm{~s}$. Hence, on time $\tau_{e}$, the pressure field is well established. As the Reynolds number in these experiments is typically large $\left(\operatorname{Re} \sim 10^{3}\right)$ the flow is inviscid. Since moreover $I \gg 1$ (i.e. $\partial \mathbf{u} / \partial t \gg(\mathbf{u} \cdot \nabla) \mathbf{u}$ ), the impulsive acceleration of the drop during the pulse follows

$$
\frac{\partial \mathbf{u}}{\partial t} \approx-\frac{1}{\rho} \nabla p,
$$

with $\mathbf{u}(r, \theta, \phi)$ the velocity and $p$ the pressure inside the drop relative to the ambient pressure. Incompressibility $(U \ll c)$ implies, upon taking the divergence of Eq. (6.5), that the pressure field is harmonic:

$$
\Delta p=0 .
$$

The velocity field just after the pressure pulse is then obtained by integration of Eq. (6.5) over time

$$
\mathbf{u} \approx-\frac{1}{\rho} \nabla \int_{0}^{\tau_{e}} p(\tau) \mathrm{d} \tau=-\frac{\tau_{e}}{\rho} \nabla p, \quad \tau_{e} \leq t \ll \tau_{i},
$$


where $p$ now refers to the time-averaged pressure inside the drop during the pulse and is therefore only a function of space. From momentum conservation (6.4) it follows that the drop speed $U$ scales as

$$
U \sim \frac{p_{e} \tau_{e}}{\rho R_{0}} .
$$

From now on, we use the scaled time $t / \tau_{e}$, radial coordinate $r / R_{0}$, pressure $p / p_{e}$, and velocity $\rho R_{0} \mathbf{u} / p_{e} \tau_{e}$. Pressure scale $p_{e}$ can be obtained from the laser-pulse energy by combining Eqs. (6.8) and (6.1).

The shape of the pressure pulse $f(\theta)$ arises as the boundary condition on the drop surface

$$
p(r=1, \theta)=f(\theta),
$$

which we normalize such that the axial momentum is equal to one, i.e.

$$
\frac{4}{3} \pi U=\int_{A} f(\theta) \mathbf{e}_{z} \cdot \mathrm{d} \mathbf{A}=2 \pi \int_{0}^{\pi} f(\theta) \cos \theta \sin \theta \mathrm{d} \theta=1 .
$$

This choice sets the (dimensionless) center-of-mass velocity of the drop $U=$ $3 /(4 \pi)$ and the associated translation kinetic energy

$$
E_{\mathrm{k}, \mathrm{cm}}=\frac{2}{3} \pi U^{2}=\frac{3}{8 \pi},
$$

independently of the choice of $f(\theta)$.

To solve the Laplace equation (6.6) in spherical coordinates we decompose the pressure field into Legendre polynomials $P_{\ell}$

$$
p(r, \theta)=\sum_{\ell=0}^{\infty} A_{\ell} r^{\ell} P_{\ell}(\cos \theta)
$$

which coefficients

$$
A_{\ell}=\frac{2 \ell+1}{2} \int_{0}^{\pi} f(\theta) P_{\ell}(\cos \theta) \sin \theta \mathrm{d} \theta,
$$

are obtained by the projection of the boundary condition (6.9). From Eq. (6.10) one observes that $A_{1}=U$.

The solution (6.12) and (6.13) can now be used to describe the drop response to any pressure- and hence any laser-beam profile. The corresponding velocity field is computed from Eq. (6.7). While by convention $E_{\mathrm{k}, \mathrm{cm}}$ does not depend on the pressure-pulse shape, the total amount of kinetic energy that has to be put into the drop to acquire this propulsion does. It is given by

$$
E_{\mathrm{k}}=\frac{1}{2} \int_{V} \mathbf{u}^{2} \mathrm{~d} V=\pi \int_{0}^{1} \int_{0}^{\pi}\left(u_{r}^{2}+u_{\theta}^{2}\right) r^{2} \sin \theta \mathrm{d} \theta \mathrm{d} r,
$$


with $V$ the drop volume. As we will see in $\S 6.3 .1$, it is convenient to define the partition

$$
\frac{E_{\mathrm{k}, \mathrm{d}}}{E_{\mathrm{k}}}=1-\frac{E_{\mathrm{k}, \mathrm{cm}}}{E_{\mathrm{k}}}
$$

between the deformation kinetic energy of the drop $E_{\mathrm{k}, \mathrm{d}}$ (i.e. the kinetic energy remaining in the co-moving frame) and the total kinetic energy (6.14).

\subsubsection{Boundary-integral simulations}

The analysis above applies when the drop shape does not deviate too much from a sphere $\left(t \sim \tau_{e} \ll \tau_{i}\right)$. To obtain the details of the subsequent dropshape evolution, one needs to solve the axisymmetric potential flow problem in the deforming shape. To this end, we employ the boundary-integral (BI) method described by Gekle et al. [82] and Bergmann et al. [81], which has already been successfully used to study drop deformation during mechanical impact [83], as well as that due to a laser impact in $\S 4$. BI is a powerful method to study the drop dynamics at later times $t \sim \tau_{i}$, when the drop shape changes significantly.

\subsection{Results}

We will now use the analytical model and BI simulations to explore the role of the laser-pulse shape, i.e. of the pressure-pulse shape, on the deformation of the drop. Indeed, the pressure boundary condition (6.9) introduced above is the actual pressure on the drop surface, which is typically proportional to the local laser fluence weighted by the cosine of the incident angle of the incoming rays on the drop surface. Typical laser-beam profiles used in experiments have a Gaussian or flat-top (uniform) shape. We consider a Gaussian pulse with a finite arbitrary width in $\S 6.3 .1$, the limits of a perfectly focused beam in $\S 6.3 .2$ and that of a uniform laser-beam profile, i.e. a cosine pressure pulse applied on one side of the drop, in $\S 6.3 .3$.

\subsubsection{Gaussian laser-beam profile}

For simplicity, we first consider a pressure pulse that applies over the entire drop surface. The effect of restricting the interaction to the side that is actually illuminated by the laser will be discussed in $\S 6.3 .3$. Since our aim is to understand the influence of the laser focus on the drop-shape evolution we also neglect the angular dependence $\cos \theta$ of the pressure profile. The 
Gaussian-shaped pressure boundary condition (6.9) then reads

$$
f(\theta)=c \exp \left[-\theta^{2} /\left(2 \sigma^{2}\right)\right],
$$

where $\sigma$ is a measure for the width of the pulse and the prefactor

$$
c=\frac{2 \sqrt{2}}{\sigma \pi^{3 / 2} \exp \left[-2 \sigma^{2}\right]\left(2 \operatorname{Erfi}[\sqrt{2} \sigma]-\operatorname{Erfi}\left[\frac{i \pi+2 \sigma^{2}}{\sqrt{2} \sigma}\right]-\operatorname{Erfi}\left[\frac{-i \pi+2 \sigma^{2}}{\sqrt{2} \sigma}\right]\right)}
$$

ensures the normalization (6.10). The resulting coefficients (6.13) are calculated by numerical integration. The convergence of series (6.12) depends on the value of $\sigma$, but in general 20 terms are sufficient to obtain accurate results (except in the limit $\sigma \rightarrow 0$, which has to be treated separately and will be discussed in $\S 6.3 .2)$.

\subsubsection{Global features}

We explore the effect of the focusing of the laser beam on the drop deformation by varying the pulse width $\sigma$, thereby mimicking the situation shown in Fig. $6.1(\mathrm{~b}-\mathrm{d})$. In Fig. 6.4 we show a plot of the resulting pressure and velocity fields inside the drop for a uniform pressure pulse $(\sigma=\pi / 4)$ and a more focused one ( $\sigma=\pi / 8$ ). In these (and the following) plots, the series solution (6.12) is cut after 20 terms. The velocity fields shown in Fig. 6.4 are in the co-moving frame: we subtracted the center-of-mass velocity of the drop to clearly illustrate the deformation of the drop during its translational motion. The analytical solution $(6.7,6.12)$ is strictly valid only as long as the domain is spherical. However, we can obtain a first-order approximation of the deformed drop shape shortly after the pressure kick by advecting the material points on the drop surface. The drop surface at time $t$ is then given by $\mathbf{r}_{\mathbf{d}}(\theta, t)=$ $\mathbf{e}_{\mathbf{r}}+\left[u_{r}(1, \theta) \mathbf{e}_{\mathbf{r}}+u_{\theta}(1, \theta) \mathbf{e}_{\theta}\right] I^{-1} t$, with I given by Eq. (6.3); see Fig. 6.4 (c) (blue dashed lines). This mere extrapolation must of course only be considered for qualitative and illustrative purposes. For a quantitative prediction, one needs to consider hydrodynamic interaction and solve for the pressure (6.6) in the deformed drop, which is done in the BI simulations. In the first stages (during which the drop is close to spherical) the BI simulations are in perfect agreement with the analytics. At later times, naturally, stronger deviations are observed and the simulation results are required to get insight into the drop-shape evolution. A few drop contours obtained from BI at these later times for a Weber number of 790 are shown in Fig. 6.4 (c) (red solid lines). Note that the Weber number used in the simulation does not affect the drop shape at early times $\left(t \ll \tau_{c}\right)$, but only sets the absolute propulsion speed and 
(a.1)

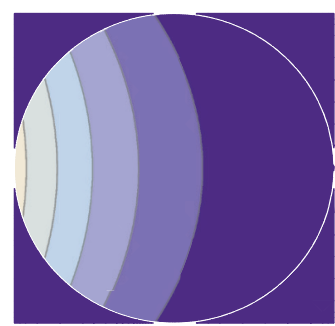

(b.1)

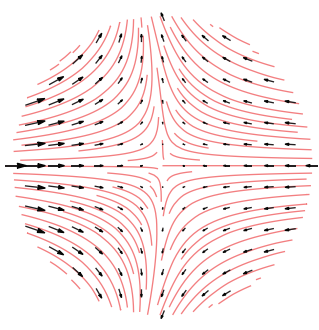

$t / \tau_{c} \ll \mathrm{We}^{-1 / 2}$ (a.2)

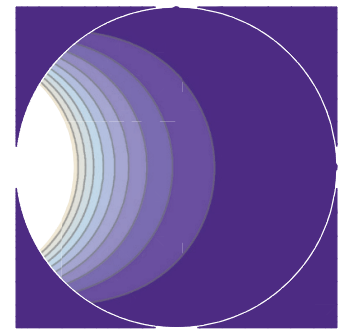

$$
t / \tau_{c} \ll \mathrm{We}^{-1 / 2}
$$

(b.2)

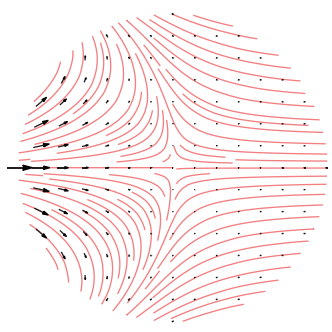

$t / \tau_{c}=0.0021$

(c.2)

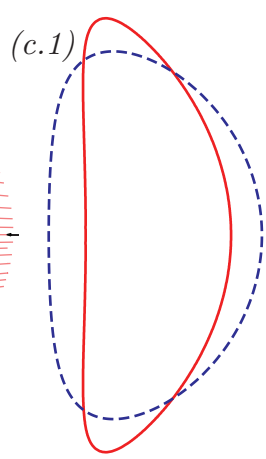

$t / \tau_{c}=0.021$
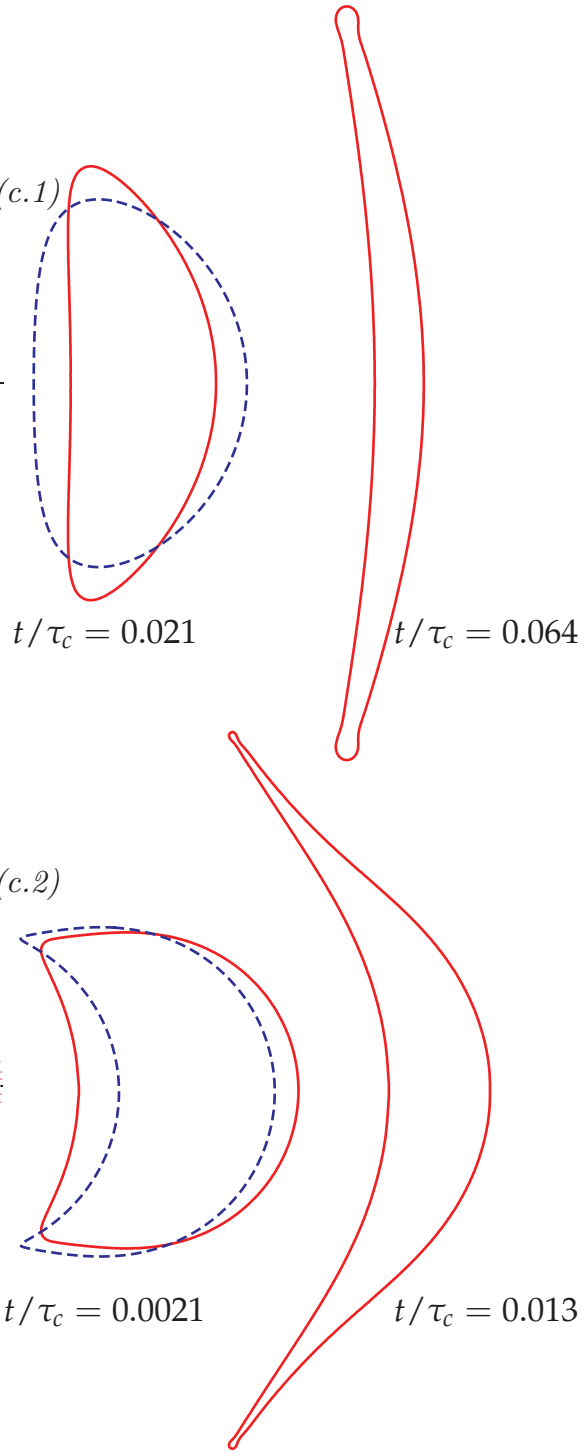

Figure 6.4: Response of a drop to Gaussian pressure pulses. (a) Iso-pressure lines inside the drop at early times $\left(t \leq \tau_{e}\right)$ for Gaussian pressure pulses with $\sigma=\pi / 4$ (a.1) and $\sigma=\pi / 8$ (a.2). (b) Streamlines of the early-time velocity field $\left(t / \tau_{c} \ll \mathrm{We}^{-1 / 2}\right)$ in the co-moving frame (drop center-of-mass velocity subtracted). (c) Drop contours illustrating the evolution of the deformation in the analytical model (blue dashed lines) obtained by advecting the material points on the drop surface (see text) and in the BI simulations (red solid lines drawn on the same scale as in $(a, b), W e=790)$. Note that the expansion is much faster for $\sigma=\pi / 8$ (c.2) than for $\sigma=\pi / 4$ (c.1), the contours being represented earlier in the latter case. 
expansion rate of the drop. As we will explain below, the relative partitioning between propulsion speed and expansion rate also remains unaffected.

From Fig. 6.4 we observe that an unfocused pulse leads to a velocity field that is almost symmetric around the vertical mid-plane (Fig. 6.4(b.1)). As a consequence, the eventual drop shape that will result from this pressure pulse is almost symmetric and flat, as is indeed observed in the BI results in Fig. 6.4 (c.1) and (to some extent) in our experimental results in Fig. 6.1 (b). By contrast, a focused pulse naturally leads to more curved iso-pressure lines and the eventual drop will also be more curved (Fig. 6.4 (c.2)), which agrees with our experimental observations in Fig. $6.1(\mathrm{c}, \mathrm{d})$. The BI results show that at later times $\left(t>\tau_{i}\right.$ and hence $\left.t / \tau_{c}>\mathrm{We}^{-1 / 2}\right)$, the drop deforms into a thin sheet bordered by a rim. For the unfocused pulse ( $\sigma=\pi / 4$, Fig. 6.4 (c.1)) this sheet is relatively flat and has an approximately uniform thickness, except for the rim itself. For the focused pulse ( $\sigma=\pi / 8$, Fig. 6.4 (c.2)) the resulting sheet has a stronger curvature with a clearly non-uniform thickness, and the expansion is much faster than for the focused pulse (note the difference in timescales between Fig. 6.4 (c.1) and (c.2).

In the BI simulations, the recession of the sheet edge eventually leads to the formation of undamped surface waves and a Bernoulli suction that results into the successive detachments of liquid rings from the edge. This pinch-off is an artifact of the simulation caused by the lack of viscous damping and the assumption of axial symmetry, as discussed by Peters et al. [112], and is clearly irrelevant to the physical fragmentation processes that actually occur. This artifact however has a negligible influence on the early-time expansion and evolution of the sheet thickness away from the rim. We therefore use the simulations until the first pinch-off event occurs.

\subsubsection{Kinetic-energy partition: deformation versus translation}

We now use the analytical results $(6.7,6.12)$ to quantify the effect of focusing the laser on the expansion rate of the drop relative to its propulsion velocity. Figure 6.5 shows the kinetic-energy partition (deformation to total kineticenergy ratio) (6.15) as a function of the pulse width $\sigma$. We also plot estimates for the energy partition obtained from the three experimental cases shown in Fig. 6.1 (b-d) and from the data of $\S 4$ (black circles). In appendix $\S 6$.B we explain in detail the (non-trivial) steps that are taken to obtain these estimates from the experimental data. For comparison, we applied the same method to the BI simulations (red squares), which confirms the validity of our method (see appendix §6.B for further discussion). Given the uncertainties in the experimental estimates in particular for the focused laser pulse, as discussed in appendix $\S 6$.B (and which also apply to the estimated Weber numbers in 


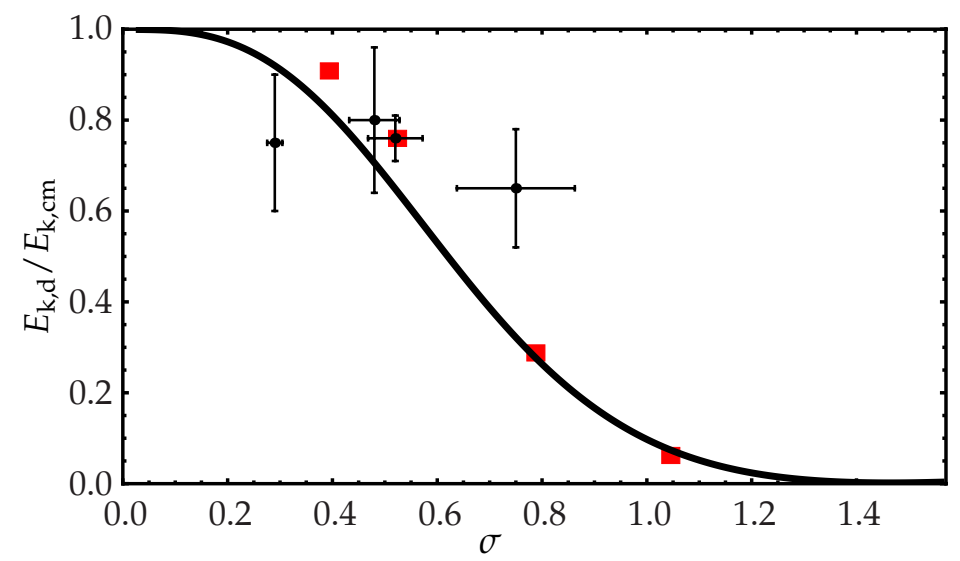

Figure 6.5: Kinetic-energy partition (6.15) as a function of the pulse width $\sigma$. For a tightly focused beam (small $\sigma$ ) almost all the energy goes into deforming the drop without translating it. For an unfocused pulse the drop only translates but hardly deforms. The black dots are experimental data obtained, by a method described in appendix $\S 6 . \mathrm{B}$, from the three events shown in Fig. $6.1(\mathrm{~b}-\mathrm{d})$ and from the experiments in $\S 4$ (four realizations of $\sigma=\pi / 6 \simeq 0.52$ ). The red squares are obtained from BI simulations using the same method to estimate the energy partition as in the experiments (see appendix §6.B).

Fig. $6.1(\mathrm{~b}-\mathrm{d})$ ), we cannot expect a quantitative agreement with theory. However, Fig. 6.5 shows that the experimental data points qualitatively confirm the theoretical prediction: the more focused the laser pulse, the more energy is used to deform the drop rather than to translate it.

Figure 6.5 shows that for a tightly focused beam (small $\sigma$ ) almost all the energy goes into deforming the drop and hardly any into translating it: the energy ratio $E_{\mathrm{k}, \mathrm{d}} / E_{\mathrm{k}} \rightarrow 1$ as $\sigma \rightarrow 0$. Indeed, the total kinetic energy required to maintain a constant center-of-mass speed diverges as the pressure pulse becomes more localized. We will discuss the limiting case when the pressure pulse comes down to a Dirac-delta pulse in more detail in $\S 6.3 .2$. By contrast, when the pulse is very broad (large $\sigma$ ) the drop experiences a pressure from all sides, such that it does not deform but only translates and $E_{\mathrm{k}, \mathrm{d}} / E_{\mathrm{k}} \rightarrow 0$. Note again that $\sigma \ll 1$ does not represent a large directional laser beam applying only on one side of the drop, which will be considered below, but rather an isotropic illumination of the drop. In fact, the Gaussian pressure pulse that is the most relevant to a uniform laser-beam profile (see §6.3.3) has $\sigma \simeq 0.73$, which is fairly unidirectional and close to the $f(\theta) \propto \cos \theta$ profile due to the local incidence of the laser on the curved drop surface.

Figure 6.5 shows that a focused pressure pulse leads to a stronger drop deformation. This does not necessarily mean that the drop will also experience 
a larger lateral expansion, since the energy could be used to deform the drop into a strongly curved shape only (i.e. to pierce the drop). To get a feeling for how much the actual expansion rate of the drop depends on the laser focus, we plot the maximum lateral expansion velocity $U_{x, \max }$ (see the inset for an illustration) at the drop surface as a function of $\sigma$ in Fig. 6.6 (a). One sees that a more focused pulse does not only lead to a larger deformation but also to a larger lateral expansion: the smaller $\sigma$, the larger $U_{x, \max }$. In Fig. $6.6(\mathrm{~b})$ we show at which position along the drop surface (in terms of azimuthal coordinate $\theta$ ) this maximum expansion velocity is observed. For a focused pulse it is observed closer to the laser axis $(\theta=0)$, whereas for an unfocused pulse it is closer to the poles of the drop $(\theta= \pm \pi / 2)$.

The faster initial expansion rate for a focused pressure pulse is confirmed by the simulations. Figure 6.7 shows drop contours from the BI simulations for four different pulse widths, from which we derive the (projected) sheet radius $R$ and thickness $h$ (measured at the center of the drop, see inset Fig. $6.8(b)$ ). Indeed, in Fig. 6.8 we observe that a smaller $\sigma$, i.e. a more focused pulse, corresponds to a faster lateral expansion and a faster decrease in the sheet thickness. We therefore conclude that in order to get a maximally expanded sheet with a minimal thickness one needs to focus the laser pulse to a tight spot (spot size $\ll R_{0}$ ), with again the consequence that this maximally expanded sheet is strongly curved and has a non-uniform thickness (bottom row Fig. 6.7).
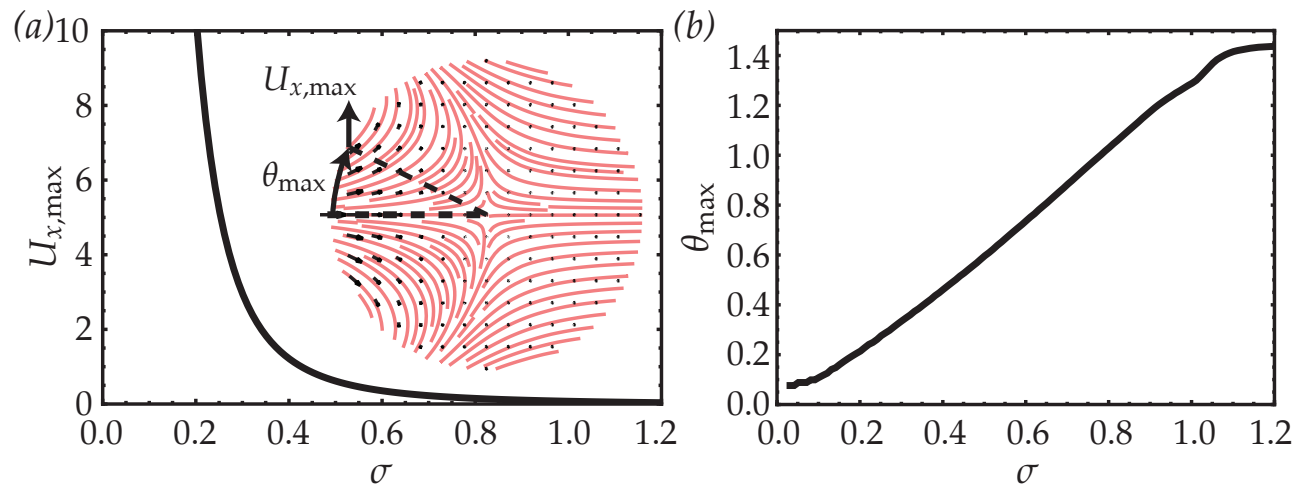

Figure 6.6: (a) Maximal expansion velocity $U_{x, \max }$ (in the $x$-direction) along the drop surface as a function of $\sigma$ for $t / \tau_{c} \ll \mathrm{We}^{-1 / 2}$. The more focused the pulse, the faster the drop expands. The inset shows the velocity field in the co-moving frame for $\sigma=\pi / 8$, and sketches $U_{x, \max }$ and its angular location $\theta_{\max }$. (b) $\theta_{\max }$ as a function of $\sigma$. For a focused pulse the maximal expansion velocity is observed around $\theta=0$, i.e. on the pulse axis of symmetry. No data is shown for $\sigma \rightarrow 0$ and $\sigma \rightarrow \pi / 2$ since in these limits the series (6.12) does not converge or the deformation velocity becomes negligible, respectively. 


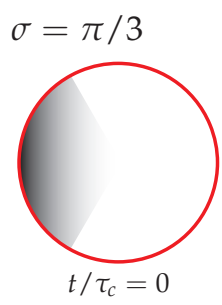

$\sigma=\pi / 4$

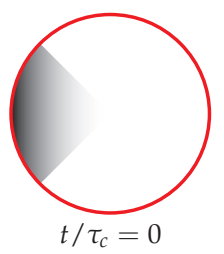

$\sigma=\pi / 6$

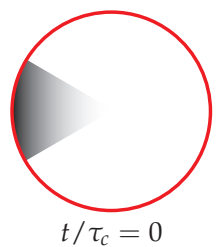

$\sigma=\pi / 8$

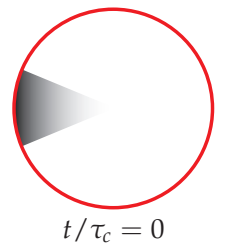

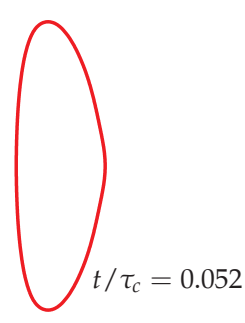
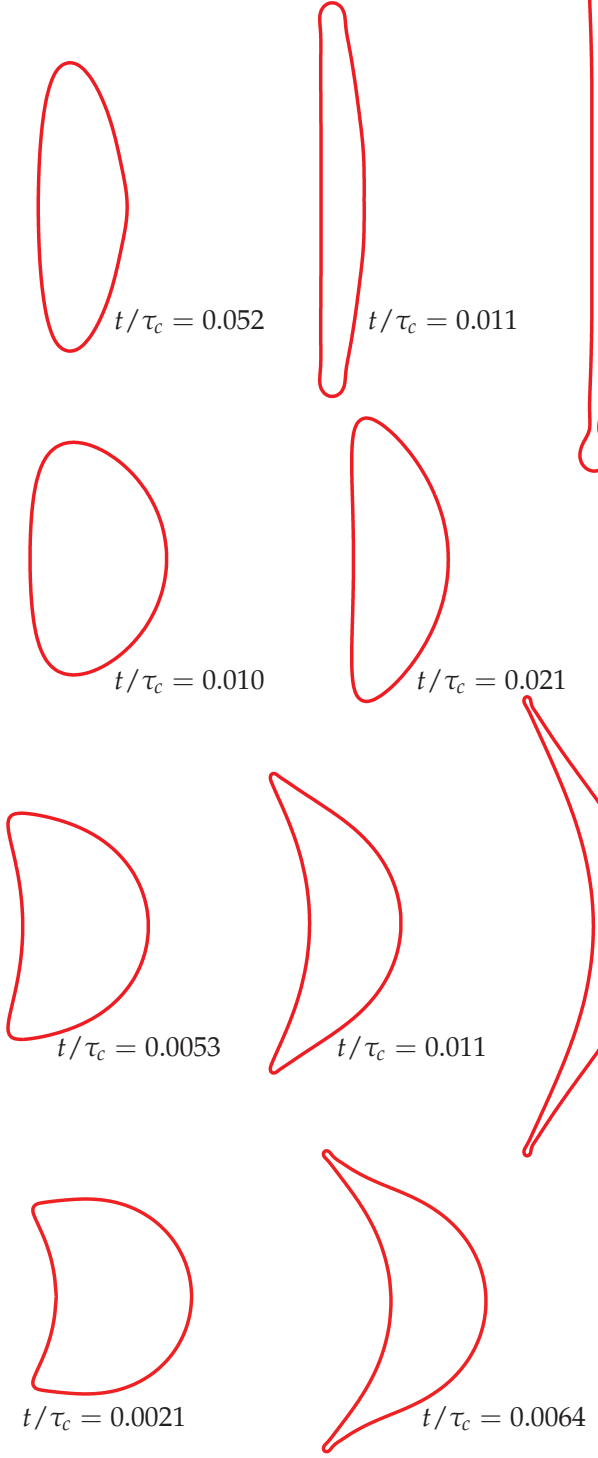

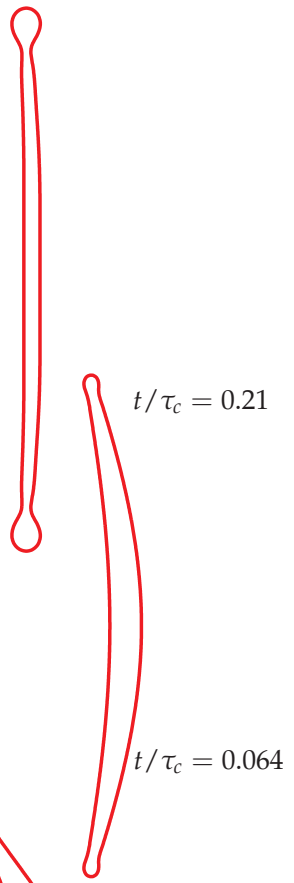

$t / \tau_{c}=0.022$

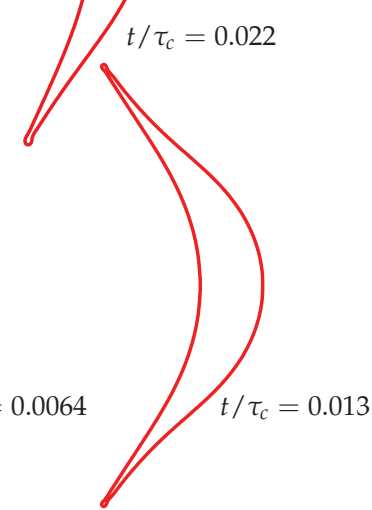

Figure 6.7: Sequences of drop contours obtained from the BI simulations illustrating the drop shape evolution for We $=790$ and four different pulse widths $\sigma=\pi / 3, \pi / 4, \pi / 6$ and $\pi / 8$, from top to bottom. Clearly, a more focused laser beam (smaller $\sigma$ ) leads to a larger expansion rate, a thinner sheet with a less uniform thickness and a more curved drop shape. Each sequence is sampled at different times to accommodate the different expansion rates. 

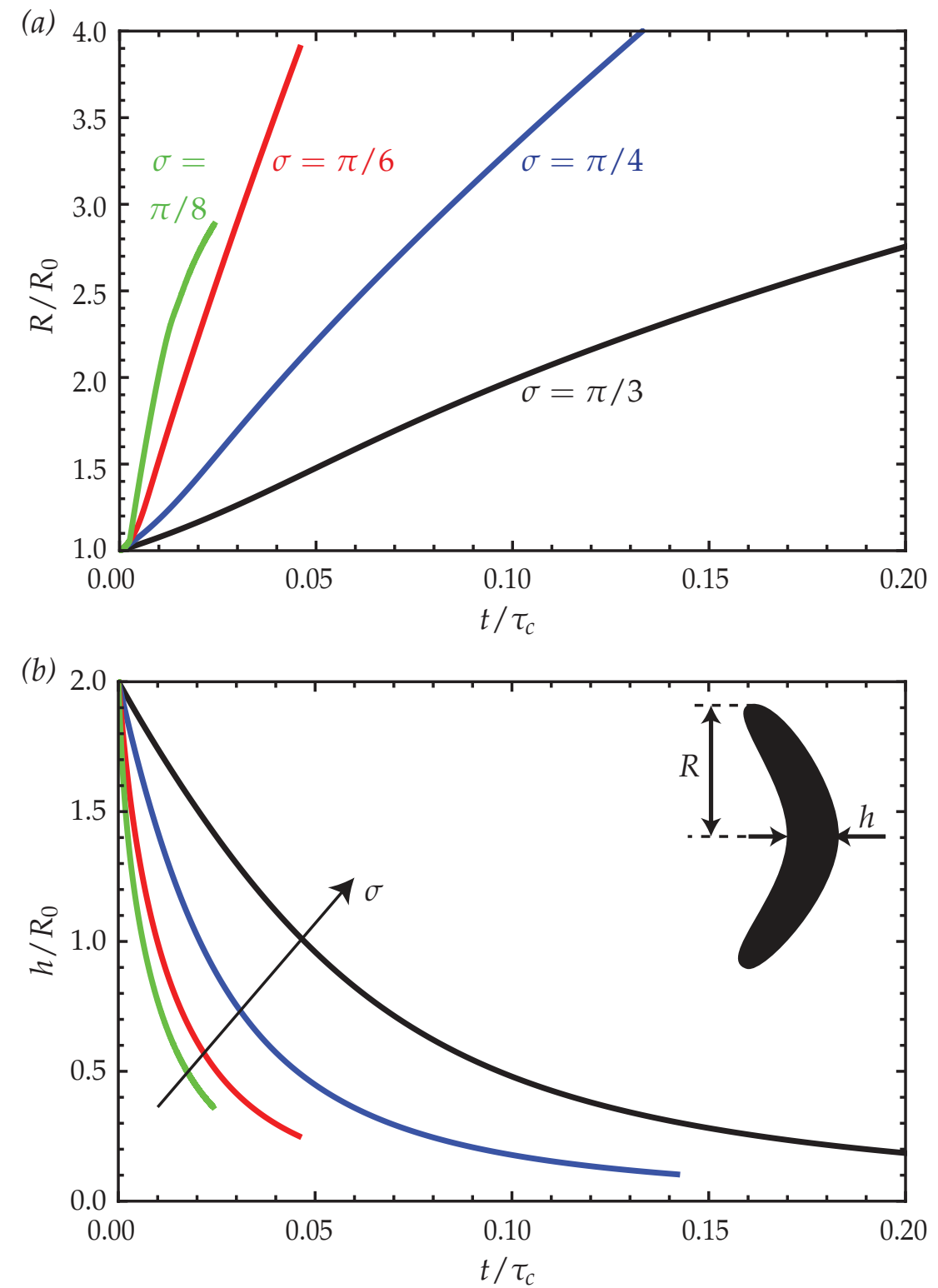

Figure 6.8: Sheet radius $R(t)$ (a) and thickness in the center $h(t)$ (b) extracted from the BI simulations shown in Fig. 6.7 (We $=790$ and $\sigma=\pi / 8, \pi / 6, \pi / 4$, and $\pi / 3$ ). 


\subsubsection{A perfectly focused laser pulse: the limit $\sigma \rightarrow 0$}

In the limit when the size of the laser pulse becomes negligibly small with respect to the drop size $(\sigma \rightarrow 0)$ the pressure pulse on the drop surface approaches

$$
f(\theta) \rightarrow \delta(\theta),
$$

where $\delta$ stands for the Dirac-delta distribution and the series (6.12) diverges. The exact solution to Eq. $(6.6,6.18)$ can however be obtained from a different approach. For $\sigma \ll 1$ the curvature of the drop surface is no longer relevant and one recovers the response of an infinite half-space to a Dirac-delta pulse. To model this situation we adopt a cylindrical coordinate system $(r, z)$, with the positive $z$-coordinate pointing into the liquid and $z=0$ corresponding to the liquid-air interface, see Fig. 6.9.

The boundary conditions for the Laplace equation (6.6) in a half-space now read

$$
\begin{aligned}
& p(r, z) \rightarrow 0 \text { for } r, z \rightarrow \infty, \\
& p(r, 0)=\frac{\delta(r)}{2 \pi r} .
\end{aligned}
$$

Hence, the pressure diverges at the origin, but the total force applied to the drop remains finite and equal to unity. The solution to the Laplace equation (6.6) with boundary conditions $(6.19,6.20)$ is obtained by taking the Hankel transform of Eq. (6.6) in $r$ [113, § 6.7] from which we find, using Eq. (6.20)

$$
p(r, z)=\int_{0}^{\infty} s p(s, 0) \mathrm{d} s \int_{0}^{\infty} k J_{0}(k r) J_{0}(k s) e^{-k z} \mathrm{~d} k=\frac{1}{2 \pi z^{2}\left(1+(r / z)^{2}\right)^{3 / 2}} .
$$

The velocity field is then obtained from Eq. (6.7):

$$
\begin{aligned}
& u_{r}(r, z)=\frac{3 r z}{2 \pi\left(r^{2}+z^{2}\right)^{5 / 2}} \\
& u_{z}(r, z)=-\frac{r^{2}-2 z^{2}}{2 \pi\left(r^{2}+z^{2}\right)^{5 / 2}},
\end{aligned}
$$

and diverges as $\epsilon^{-3}$ for $\epsilon=r, z \rightarrow 0$. As a consequence, the total kinetic energy contained in the half-space is non-integrable. We therefore calculate the kinetic energy excluding a region of size $\epsilon$ around the origin

$$
E_{\mathrm{k}}=\lim _{\epsilon \rightarrow 0} \pi \int_{0}^{\infty} \int_{\epsilon}^{\infty}\left(u_{r}^{2}+u_{z}^{2}\right) r \mathrm{~d} r \mathrm{~d} z=\lim _{\epsilon \rightarrow 0} \frac{3}{128 \epsilon^{3}} .
$$




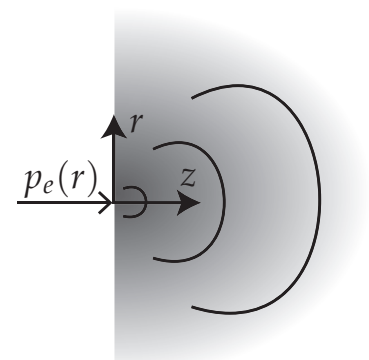

Figure 6.9: Situation for a tightly focused laser beam. The drop surface curvature becomes irrelevant and the pressure pulse $p_{e}$ comes down to a Dirac-delta distribution (6.18) applying at the surface of an infinite half-space with cylindrical coordinates $r, z$.

Hence, the total kinetic energy diverges as $\epsilon^{-3}$ for $\epsilon \rightarrow 0$ and is contained in a tiny volume of size $\epsilon^{3}$, which is small compared to the drop size. In practice, for a Gaussian of finite width (6.16) one can interpret $\epsilon=\sigma$ in the limit $\sigma \rightarrow 0$, and hence the drop kinetic energy diverges as $\sigma^{-3}$. We verified that the total kinetic energy obtained from the series solution (6.12) indeed exhibits the same divergence. Since the translation kinetic energy of the drop is constant $E_{\mathrm{k}, \mathrm{d}} / E_{\mathrm{k}} \rightarrow 1$ as $\sigma \rightarrow 0$, as was already observed in Fig. 6.5.

The same conclusion can be reached from a simple scaling argument. We apply a finite force $F=\int p \mathrm{~d} A$ to the drop. In the limit $\sigma \rightarrow 0$ the characteristic area on which this force acts scales as $\sigma^{2}$, such that the local pressure $p \sim F / \sigma^{2}$ diverges. From momentum conservation the velocity field inside the drop scales as $u \sim F \tau / \rho \sigma^{3}$, such that the kinetic energy, which is used to deform a volume of size $\sigma^{3}$, scales as

$$
E_{\mathrm{k}} \sim \rho u^{2} \sigma^{3}=F^{2} \tau^{2} / \rho \sigma^{3}
$$

and hence diverges as $\sigma^{-3}$ for $\sigma \rightarrow 0$, while the ratio $E_{\mathrm{k}, \mathrm{d}} / E_{\mathrm{k}}$ remains finite and approaches one.

\subsubsection{A one-sided uniform laser pulse yields a flat drop}

A flat, symmetric drop shape can obviously be obtained by impacting the drop symmetrically with two laser beams. We will however see now that a flat shape can also be obtained with a uniform (or flat-top) laser-beam profile impacting the drop from one side only.

As discussed above, a uniform laser-beam profile results in a cosine-shaped 
pressure profile on the drop surface

$$
f(\theta)=\frac{3}{2 \pi} \cos \theta H(\pi / 2-\theta),
$$

where the Heaviside function $H$ restricts the interaction to the illuminated side of the drop. The coefficients (6.13) can be obtained exactly and read

$$
\begin{aligned}
A_{\ell} & =\frac{3(2 \ell+1)}{4 \pi} \int_{0}^{\pi / 2} P_{\ell}(\cos \theta) \cos \theta \sin \theta \mathrm{d} \theta \\
& =\frac{3(1+2 \ell)}{16 \sqrt{\pi} \Gamma(3 / 2-\ell / 2) \Gamma(2+\ell / 2)},
\end{aligned}
$$

from which we find

$$
p(r, \theta)=U r \cos \theta+\frac{3}{16 \sqrt{\pi}} \sum_{n=0}^{\infty} \frac{1+4 n}{\Gamma(3 / 2-n) \Gamma(2+n)} r^{2 n} P_{2 n}(\cos \theta),
$$

which involves only the even Legendre polynomials. The series (6.28) converges. However, despite the fact that the pressure field itself is continuous, its first derivative with respect to $\theta$ and hence the velocity $u_{\theta}$ exhibit a discontinuity in $\theta=\pi / 2$ caused by the restriction of the pressure boundary-condition (6.26) to the front of the drop. In reality, this discontinuity will be smoothed by viscosity. The resulting pressure (6.28) and velocity (6.7) fields are shown in Fig. 6.10. We use the velocity field to obtain the energy ratio

$$
\frac{E_{\mathrm{k}, \mathrm{d}}}{E_{\mathrm{k}}} \approx 0.35 \text {. }
$$

Notice that this energy ratio can also be reached with a Gaussian pressure pulse with $\sigma \approx 0.73$ (see Fig. 6.5).

The velocity field in the co-moving frame shown in Fig. 6.10 (b) displays a striking feature: it is symmetric not only around the horizontal axis (owing to the axi-symmetry of the pressure pulse), but also around the vertical axis. This means that the drop eventually deforms into a perfectly flat, symmetric shape even though the laser impact is only one-sided, as the BI results in Fig. 6.10 (c) confirm. One can understand this symmetry in the velocity field by inspecting the expression for the radial velocity after subtraction of the center-of-mass velocity $U$

$$
u_{r}(r, \theta)=-\frac{3}{16 \sqrt{\pi}} \sum_{n=1}^{\infty} \frac{2 n(1+4 n)}{8 \Gamma(3 / 2-n) \Gamma(2+n)} r^{2 n-1} P_{2 n}(\cos \theta) .
$$

Realizing that $P_{2 n}(x)=P_{2 n}(-x)$, one sees that this velocity field is indeed symmetric. More heuristically one may note that a cosine pressure pulse 
(a)

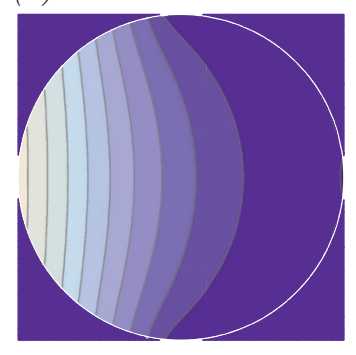

(b)

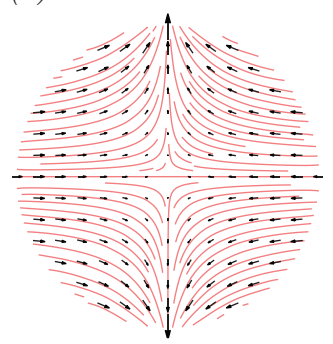

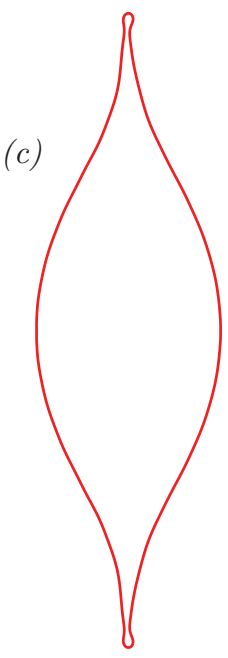

$t / \tau_{c}=0.021$

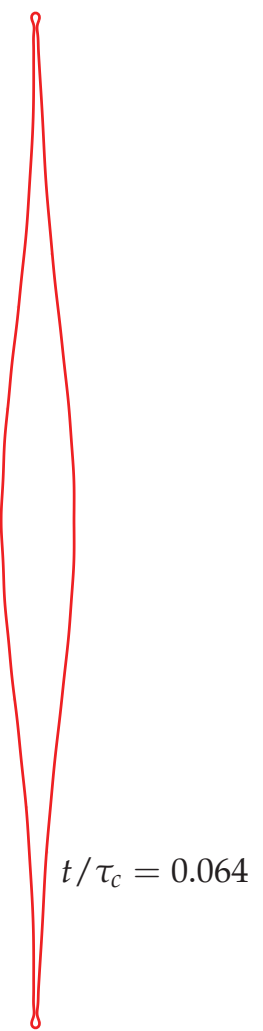

Figure 6.10: Symmetric deformation obtained for a uniform laser profile (cosineshaped pressure pulse (6.26)). (a) Iso-pressure lines. (b) Streamlines of the velocity field in the co-moving frame. (c) Sequence of drop contours from the BI simulations for We $=790$ (same scale as in $(\mathrm{a}, \mathrm{b})$ ). Note that the discontinuity in $u_{\theta}$ cannot be fully resolved by both the analytics and the numerics due to the finite number of terms used in the series (6.28) and the finite grid size, respectively.

accommodates the drop shape: both the pressure and the local thickness of the drop are proportional to $\cos \theta$ from which each slice of the drop acquires the same axial velocity. It can actually be proven that a cosine pressure pulse is the only one-sided profile that results in a symmetric (flat) drop, which we do in appendix $\S 6$.A.

\subsection{Late time dynamics: the thin sheet limit}

Up to now we have been concerned with the early time $t \sim \tau_{e}$ of the dynamics, when the drop is still spherical and the influence of surface tension is negligible. The BI simulations allowed us to extend the description to later times, close 
to the maximal extension of the drop. We now consider the late-time regime $t \sim \tau_{c}$ when the drop expands into a thin sheet and subsequently recedes.

\subsubsection{Problem formulation \& solution}

We follow the same approach as Villermaux and Bossa [80] have used to describe the surface-tension limited expansion of a rain drop due to aerodynamics effects. We thus describe the dynamics of a flat, thin sheet in a frame co-moving with the center-of-mass velocity, see Fig. 6.11. The sheet has a time-dependent, uniform thickness $h(t) \ll R(t)$ (with $R(t)$ the timedependent drop radius) and a given initial kinetic energy, which is precisely that determined in the early-time model. This follows from the inviscid flow considered here: the kinetic energy is conserved as long as surface tension does not influence the drop deformation. Since we are typically interested in large Weber numbers, surface tension effects are negligible during the early stage of expansion $\left(t \sim \tau_{i} \ll \tau_{c}\right)$. Furthermore, when the drop deforms into an essentially flat sheet, such as that shown in Fig. 6.4 (a), all the kinetic energy of deformation is used to expand the drop laterally. One can therefore use the kinetic energy obtained from the early-time model as an initial condition for the thin-sheet model.

We adopt a cylindrical coordinate system $(r, z)$, with $r$ the lateral direction (in which the sheet expands) and $z$ the direction normal to the sheet surface (see Fig. 6.11). The sheet dynamics is prescribed by the axisymmetric Euler equation. In the thin-sheet approximation $(h \ll R)$ the lateral flow $u(r, t)$ in the sheet is governed by

$$
\begin{aligned}
& \frac{\partial u}{\partial t}+u_{r} \frac{\partial u_{r}}{\partial r}=-\frac{\partial p}{\partial r} \\
& r \frac{\partial h}{\partial t}+\frac{\partial}{\partial r}(r u h)=0,
\end{aligned}
$$

where all lengths are non-dimensionalised by $R_{0}$, all times by $\tau_{c}$ and the pressure by $\gamma / R_{0}$. It follows from global mass conservation that $h=4 / 3 R^{-2}$ so that using (6.32) we find

$$
u(r, t)=r \frac{\dot{R}}{R}
$$

Integration of Eq. (6.31) between $r=0$ and $r=R(t)$ gives [80]

$$
R \ddot{R}=-2[p(R)-p(0)] .
$$

For $r \ll R(t)$ the interface curvature is close to zero, whereas for $r=R$ it is approximately $2 / h(t)$, such that Eq. (6.34) reduces to

$$
R \ddot{R}=-\frac{4}{h}=-3 R^{2} .
$$




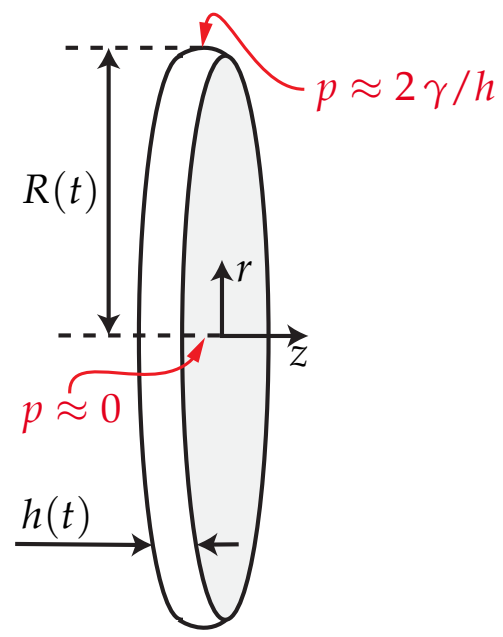

Figure 6.11: Sketch of an axisymmetric flat thin sheet with time-dependent thickness $h(t)$ and radius $R(t)$. The surface tension generates a typical Laplace pressure difference $2 \gamma / h$ between the edge and the center of the sheet, which drives the recession. The cylindrical coordinate system $(r, z)$ is indicated.

The solution reads $R(t)=a \cos \sqrt{3} t+b \sin \sqrt{3} t$, with constants $a$ and $b$ to be determined from the initial conditions. The initial radius $R(0)=1$ sets $a=1$. To derive the initial rate of expansion $\sqrt{3} b$ we use the fact that at $t=0$ the deformation kinetic energy of the sheet

$$
E_{\mathrm{k}, \mathrm{d}}^{S}=\frac{1}{2} \int_{0}^{R(t)} 2 \pi u^{2} h r \mathrm{~d} r=\frac{1}{3} \pi \dot{R}^{2}
$$

has to match the deformation kinetic energy of the drop obtained from the early-time model. In terms of the early-time kinetic-energy partition $E_{\mathrm{k}, \mathrm{d}} / E_{\mathrm{k}, \mathrm{cm}}$ we obtain

$$
E_{\mathrm{k}, \mathrm{d}}^{s}(t=0)=\pi b^{2}=\frac{2}{3} \pi\left(\frac{E_{\mathrm{k}, \mathrm{d}}}{E_{\mathrm{k}, \mathrm{cm}}}\right) \text { We. }
$$

Eliminating $b$ from Eq. (6.37) we find the solution

$$
R(t)=\cos \sqrt{3} t+\left(\frac{2}{3}\right)^{1 / 2}\left(\frac{E_{\mathrm{k}, \mathrm{d}}}{E_{\mathrm{k}, \mathrm{cm}}}\right)^{1 / 2} \mathrm{We}^{1 / 2} \sin \sqrt{3} t .
$$

This square-root dependence of the sheet radius on the Weber number is well-known for drop impact on solids in absence of friction [42, 114-116]. As already shown in $\S 4$, it is also in good agreement with experimental observations for a drop impacted by a laser. 
It is important to realize that the expanding thin sheet described here is actually subjected to hydrodynamic instabilities that may eventually cause the sheet to fragment, as Fig. 6.1 (b-d) clearly shows. First, the rapid acceleration of the drop on $\tau_{e}$ may trigger a destabilization in the sense of Rayleigh-Taylor, which could puncture the sheet, similar to what has been observed by Bremond and Villermaux [117] for sheets subjected to shock waves. Second, the rim formed at the edge of the receding sheet may develop both RayleighTaylor and Rayleigh-Plateau instabilities, as it is observed for a drop impacting a pillar [80]. A description of these instabilities is however beyond the scope of the present chapter and is left for $\S 7$.

\subsubsection{Comparison to $\mathrm{BI}$ and experiments}

To compare the thin-sheet model (6.38) to the experimental and BI results presented in $\S 4$ we first determine, using the early-time model, the energy partition that sets the initial condition (6.37). To this end, we need a description of the experimental laser-beam profile, from which we deduce the pressure boundary condition for the early-time model. We showed in $\S 4$ that the experimental beam profile is well described by a Gaussian curve (6.16) of width $\sigma=\pi / 6$. In the same study, this Gaussian pressure profile was already successfully used in BI simulations to calculate the lateral drop expansion, which suggests that irregularities in the beam profile have a negligible influence on the drop expansion. Here we use the same pressure profile in the early-time model to determine the kinetic-energy partition

$$
\frac{E_{\mathrm{k}, \mathrm{d}}}{E_{\mathrm{k}, \mathrm{cm}}}=1.8,
$$

or, in terms of the energy ratio depicted in Fig. 6.5, $E_{\mathrm{k}, \mathrm{d}} / E_{\mathrm{k}}=0.64$. This energy partition is then used in Eq. (6.37) to obtain the initial condition for the thin-sheet model.

Figure 6.12 compares the thin-sheet model (6.38) with experimental and BI results. The thin-sheet model assumes a flat drop, whereas in the experiments and BI simulations the drop is curved (for a pulse width $\sigma=\pi / 6$, see Fig. 6.7). For the comparison we therefore use the projected radius as defined in the inset in Fig. 6.8 (b). The thin-sheet model provides an accurate prediction of the expansion speed and temporal evolution of the sheet for all Weber numbers without any adjustable parameter. For $\mathrm{We}=60$ the BI and theoretical model almost completely overlap and are very close to the experimental data. Not surprisingly, at short times $\left(t / \tau_{c}<0.1\right)$ or at smaller Weber numbers, when the drop has not expanded into a thin sheet with $h \ll R$, the analytical model and $\mathrm{BI}$ simulations deviate from each other. For We $\sim 1$ the drop only oscillates 


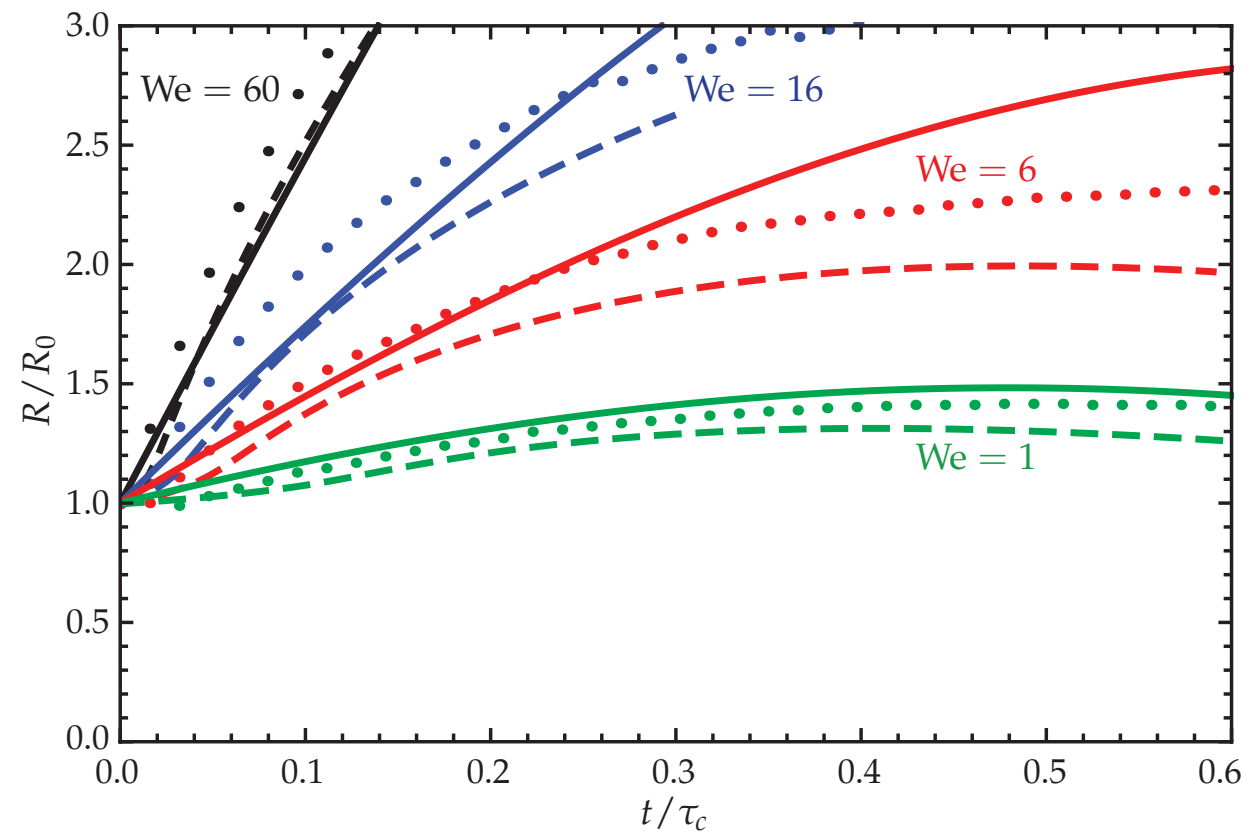

Figure 6.12: Time evolution of the sheet radius $R$ for $W e=60$ (black), We $=16$ (blue), $\mathrm{We}=6$ (red) and We $=1$ (green). The dashed lines represent the BI simulations for $\sigma=\pi / 6$ and the dots are the experimental data, both reproduced from $\S 4$. The solid lines shows the theoretical prediction based on the thin sheet model (6.38) with no adjustable parameter: the initial radius is set to unity and the initial kineticenergy partition is taken from the inertial model for a pulse width $\sigma=\pi / 6$ that matches the experimental beam profile. Experimental and BI data are shown until the fragmentation starts to influence the sheet radius or the sheet becomes locally too thin to be resolved accurately in the numerics, respectively.

around its spherical shape (see Fig. 4.3 in $\S 4$ ), and even though the differences between model and experiments for these small deformations is also small, the thin-sheet approximation is obviously no longer applicable. Moreover, in the thin-sheet model we assume that all deformation kinetic energy is used for radial expansion and the deforming sheet remains flat, whereas in experiment and BI it is slightly curved (see Fig. 6.7), which implies that some of the deformation energy is not used for radial expansion. Despite all of this, the analytical model and BI simulations quantitatively describe the experimental data.

The good agreement between the thin-sheet model and the experiments and BI simulations suggests that, although the initial expansion rate is very sensitive to the beam width (see Fig. 6.5 and Fig. 6.6) moderate curvatures and thickness variations in the sheet have little influence on the actual global 
expansion of the drop. One should however bear in mind that these moderate non-uniformities might have important consequences for the eventual sheet puncture and fragmentation.

\subsection{Conclusion}

The interaction of a laser pulse with an absorbing liquid drop can successfully be modeled by applying a recoil-pressure pulse to the drop surface. The relation between the total impulse of this recoil pressure and the laser-pulse energy is found from scaling arguments, whereas the profile of the pressure pulse can be considered, as a first approximation, to follow that of the drop surface illumination (i.e. that of the laser-beam profile weighted by its local incidence on the drop surface). Once this relation is known, the hydrodynamic response of the drop to the laser impact (propulsion, expansion and recession, possibly leading to fragmentation) is entirely captured from the drop response to the corresponding pressure pulse. This approach allows to study the response of the drop to laser pulses of different shapes and focus.

An analytical model for the impulsive acceleration when the drop is still spherical provides the early-time drop dynamics as a function of the laserpulse shape: the kinetic-energy partition inside the drop is obtained, from which we derive the amount of deformation versus translation of the drop. This yields a first-order estimate of the drop shape evolution at later times by advecting the material points on the drop surface. We find that, for a given propulsion of the drop, a maximal expansion is obtained when the laser pulse is focused into a tight spot, which results in a strongly curved sheet, while a flat symmetric sheet can only be obtained with a uniform laser-beam profile.

On the inertial and capillary timescales boundary-integral simulations reveal the details of the sheet thickness and curvature dependence on the pulse focus, until close to the maximal expansion (where the simulation breaks down). Assuming a flat drop, we derive an analytical thin-sheet model initialized with the expansion rate obtained from the early-time model. The thin-sheet model predicts the entire evolution of the sheet radius (expansion and recession) and shows a good agreement with both experimental and BI data, in particular for large Weber numbers.

The drop deformation dynamics described by the models discussed here forms the starting point to study the subsequent drop fragmentation which is observed experimentally for high-energy laser pulses (i.e. drop expansion We $\gg 1)$, see Fig. $6.1(\mathrm{~b}-\mathrm{d})$ in this chapter and also Fig. 4.3 in $\S 4$. Understanding the mechanisms behind this fragmentation will be the subject of $\S 7$. 


\section{A Only a uniform laser beam profile results in a flat drop}

In $\S 6.3 .3$ we found that a one-sided impact with a uniform laser beam (and hence a cosine-shaped pressure pulse) results in a flat symmetric drop. We demonstrate here that the uniform beam profile is in fact the only profile that gives rise to a flat drop.

A requirement for symmetry is that after subtraction of the center-of-mass speed the velocity field satisfies the property $u_{r}(r, \theta)=u_{r}(r, \pi-\theta)$. Inspecting e.g. Eq. (6.30) we see that this requires the odd coefficients in Eq. (6.13) to be equal to zero, except for $A_{1}=U$ to ensure the center-of-mass speed. Since the velocity field (6.7) is obtained from the pressure field by taking the gradient, the symmetry of the velocity field implies that the odd coefficients in the pressure field (6.12) should also be equal to zero (again except for $A_{1}$ ). Hence, the pressure pulse $f(6.9)$ needs to satisfy

$$
\begin{aligned}
& A_{1}=\frac{3}{2} \int_{-1}^{1} f(x) x \mathrm{~d} x=U, \\
& A_{2 n+1}=\frac{4 n+3}{2} \int_{-1}^{1} f(x) P_{2 n+1}(x) \mathrm{d} x=0 \text { for } n>0 .
\end{aligned}
$$

When the drop is hit by a laser pulse the recoil pressure is only exerted from one side:

$$
f(x)=g(x) H(1-x) .
$$

Hence, we need to find the functional form of $g$ such that $f$ satisfies Eq. (6.40). To this end, we express $g$ into the Legendre series

$$
g(x)=\sum_{m=0}^{\infty} d_{m} P_{m}(x)
$$

Substituting Eq. $(6.41,6.42)$ into Eq. (6.40) and evaluating the coefficients $A_{n}$ we obtain

$$
A_{2 n+1}=\frac{4 n+3}{2} \sum_{m=0}^{\infty} d_{m} \int_{0}^{1} P_{m}(x) P_{2 n+1}(x) \mathrm{d} x, \text { for } n=0,1,2 \ldots,
$$

where the integral now runs from zero to one. Using the property that

$$
\int_{0}^{1} P_{m}(x) P_{n}(x) \mathrm{d} x= \begin{cases}\frac{1}{2 m+1} & \text { if } m=n, \\ 0 & \text { if } m \neq n, m, n \text { both even or odd } \\ h_{m, n} & \text { if } m \text { even, } n \text { odd } \\ h_{n, m} & \text { if } m \text { odd, } n \text { even, }\end{cases}
$$

with $h_{m, n}=\frac{(-1)^{(m+n+1) / 2} m ! n !}{2^{m+n-1}(m-n)(m+n+1)\left[\left(\frac{1}{2} m\right) !\right]^{2}\left\{\left[\frac{1}{2}(n-1)\right] !\right\}^{2}}[118$, p. 173] we find that 


$$
A_{2 n+1}=\frac{4 n+3}{2}\left(\frac{1}{4 n+3} d_{2 n+1}+\sum_{m=0}^{\infty} d_{2 m} h_{2 m, 2 n+1}\right), \text { for } n=0,1,2 \ldots
$$

In order to satisfy Eq. (6.40) we need $A_{2 n+1}=0 \forall n>0$. From Eq. (6.45) we observe that this requirement is satisfied for all $n$ simultaneously only when $d_{m}=0$ for $m \neq 1$, i.e.

$$
g(x)=d_{1} P_{1}(x)=d_{1} \cos (x) .
$$

This implies that the only way to form a flat, symmetric drop with a onesided impact is to illuminate the drop uniformly, i.e. with a uniform or strongly defocused Gaussian laser-beam profile.

\section{B How to determine the kinetic-energy partition in experiments}

In Fig. 6.5 we showed the analytically obtained kinetic-energy partition in the drop as a function of the pulse width $\sigma$. For comparison, we also plotted the data points corresponding to the experiments shown in Fig. 6.1. Below we outline how these experimental estimates are obtained.

In case the drop expands into a flat, thin sheet all deformation kinetic energy is used for lateral expansion and we find, using Eq. (6.36), the kineticenergy partition

$$
\frac{E_{\mathrm{k}, \mathrm{d}}^{S}}{E_{\mathrm{k}, \mathrm{cm}}}=\frac{1}{2} \frac{\dot{R}^{2}}{U^{2}}
$$

and hence

$$
\frac{E_{\mathrm{k}, \mathrm{d}}^{s}}{E_{\mathrm{k}}}=\frac{\dot{R}^{2}}{\dot{R}^{2}+2 U^{2}} .
$$

The above expression is exact in case the drop expands into a flat sheet, hence for a uniform laser-beam profile. However, for the experimental data points shown in Fig. 6.5 we also used Eq. (6.48) to estimate the energy partition for more focused beam profiles. To obtain this estimate we had to extract the lateral expansion rate and the center-of-mass speed of the drop for the different cases shown in Fig. 6.1 from simultaneous high-speed front- and side-view recordings of the drop shape evolution (for details on the experimental setup the reader is referred to $\S 3$ ).

We determined the initial expansion rate $\dot{R}$ based on the first three images available in the front-view recordings by fitting ellipses to the drop shape 

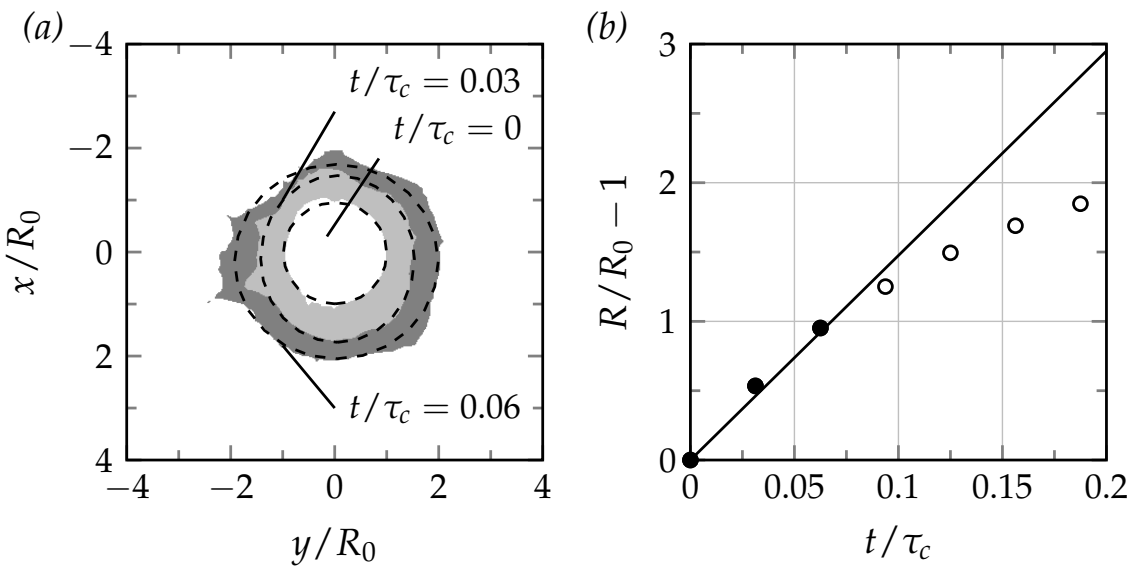

Figure 6.13: (a) Stacked front-view images (taken from the laser beam axis) of the expanding drop shown in Fig. 6.1(c). The dashed lines are the ellipses best fitting the contours, from which the equivalent radii $R$ are determined. (b) Relative expansion of the drop obtained from the views shown in (a) (•) and from later times (o). The linear fit (solid line) to the first points yields a dimensional expansion speed $\dot{R}=$ $14.6 R_{0} / \tau_{c}=4.2 \mathrm{~m} / \mathrm{s}$.

at each instant, as explained in Fig. 6.13. The selected frame rate of 10000 frames per second ensures a sufficiently rapid sampling of the expansion such that the first three data points are well described by a linear fit (Fig. 6.13 (b)). Difficulties in the determination of the actual equivalent drop radius $R$ arise when ligaments formed by the fragmentation of the drop corrupt the view (see e.g. Fig. 6.1 (b)). In our analysis, we excluded these ligaments from the ellipse-fitting.

To determine the propulsion speed $U$ of the drop we used the side-view images (see Fig. 6.14). We assumed an axisymmetric drop shape and determined the center-of-mass position $z_{c m}$ for each frame of the high-speed recordings. After the initial acceleration of the drop on the timescale $\tau_{e}$ the propulsion speed is constant and can hence be determined by a linear fit to the center-ofmass position. Since the side-view images are two-dimensional projections of the actual drop shape, they do not resolve the concavity of the drop. This introduces an uncertainty in the determination of the center-of-mass position, in particular for the more focused laser-beam profiles, where the drop evolves into a concave shape. We estimate the total error in $\dot{R} / U$ due to all the effects described above to be of the order of $20 \%$.

Finally, for each experiment the corresponding laser-pulse width $\sigma$ was determined by fitting our experimental laser-beam profiles with a Gaussian curve. Errors may arise from deviations of the beam profile from a perfect 
(a)

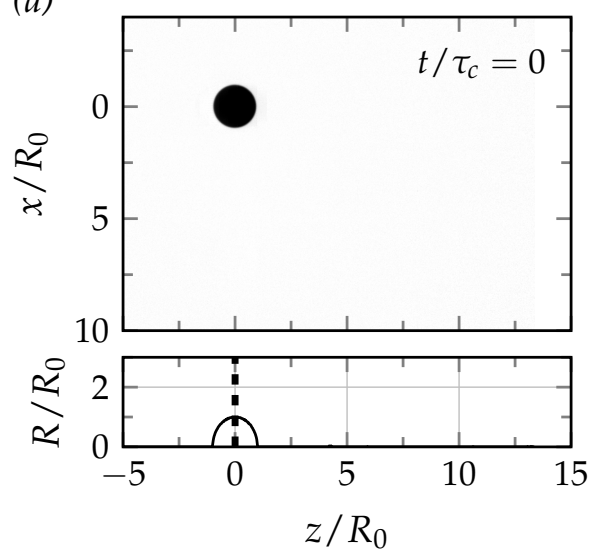

(b)

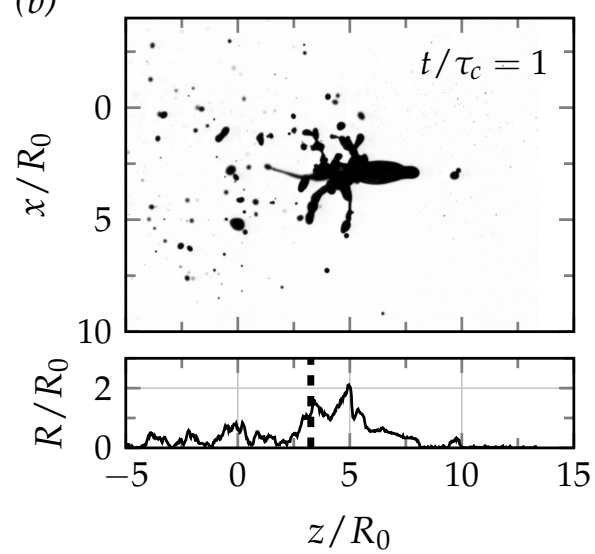

Figure 6.14: Two side-views (taken perpendicularly to the laser-beam direction) of the event shown in Fig. 6.1 (d) at (a) $t / \tau_{c}=0$ and (b) $t / \tau_{c}=1$. The top frames show the actual shadowgraphs and the bottom ones show an axisymmetric profile that was obtained by summing up pixel values in $x$-direction (i.e. collapsing all pixel values to the axis). The dashed line indicates the center-of-mass axial position $z_{c m}=\int z R^{2} \mathrm{~d} z / \int R^{2} \mathrm{~d} z$, which assumes axi-symmetry of the profile.

Gaussian, shot-to-shot variations in the laser-beam profile, and uncertainty in the drop position within the laser beam, in particular for the unfocused beams where the drop is still hit by the laser even if it is positioned slightly off-center. For the experiments shown in Fig. 6.1 we estimate the uncertainty in $\sigma$ to vary from $\sim 15 \%$ for the unfocused case to $\sim 5 \%$ for the most focused case.

To investigate the validity of our estimate for the energy partition (6.48) we use the results from the BI simulations shown in Fig. 6.7 and 6.8. In BI the center-of-mass speed is known (and constant for each value of $\sigma$ ) and the pressure profile is exactly Gaussian with a known $\sigma$. Hence, in BI the uncertainties that appear in experiment are absent and the only approximation that remains is the use of Eq. (6.48) as a measure for the energy partition. To find $\dot{R}$ in BI we determined the initial slopes of the curves in Fig. 6.8 (a), similar to what has been done for the experimental data. The resulting estimate for the energy partition is in good quantitative agreement with its theoretical prediction; see the red squares in Fig. 6.5. Small deviations $(<15 \%)$ are observed for the most focused pulses $(\sigma=\pi / 6$ and $\pi / 8)$, where the sheet is strongly curved and hence the approximation breaks down. Nevertheless, the quantitative agreement between theory and BI confirms that Eq. (6.48) is indeed a reasonable estimate of the energy partition in the range of pulse widths studied here. 


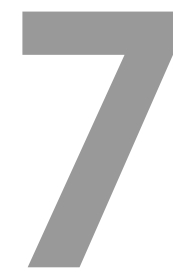

\section{Drop fragmentation by laser-pulse impact ${ }^{*, \dagger}$}

We study the fragmentation of a free-falling liquid drop that is impulsively accelerated by the impact of a laser pulse. The drop expands into a thin sheet that breaks by the radial expulsion of ligaments and the nucleation, growth, and merging of holes on the thin sheet itself. We identify two Rayleigh-Taylor mechanisms as cause of the destabilization and derive scaling laws for the characteristic time and wavenumber of destabilization for both the radial expulsion of ligaments and the hole nucleation. Combining experimental data from two liquid systems allows us to reveal how the initial laser-matter interaction affects the late-time fragmentation. The final structure of liquid ligaments is the result of an interplay between the random hole nucleation, which originates from the amplification of noise as set by the laser-matter interaction, and deterministic modulations in the local sheet thickness. The latter are a late-time consequence of the kinematics of the drop deformation and spatial variations in the laser-beam profile.

${ }^{*}$ To be submitted as: Alexander L. Klein, Dmitry Kurilovich, Henri Lhuissier, Oscar O. Versolato, Detlef Lohse, Emmanuel Villermaux, and Hanneke Gelderblom. Fragmentation of free-falling drops by laser-pulse impact.

${ }^{\dagger}$ Alexander L. Klein and Dmitry Kurilovich contributed to this chapter as part of their PhD theses, tin data is acquired by Dmitry Kurilovich and MEK data by Alexander L. Klein 


\subsection{Introduction}

The impact of a laser pulse onto a free-falling liquid drop induces a largescale deformation that eventually leads to the fragmentation of the liquid body. Figure 7.1 shows how a laser impact causes spherical drops to deform into thin, liquid sheets that subsequently break into a set of ligaments and smaller drops. Two types of breakup constitute the fragmentation process in our experiments: the radial expulsion of ligaments from a bordering rim of the sheet (Fig. 7.1 (a, c)) and the nucleation of holes on the thin sheet itself (Fig. 7.1 (b, d)). Both phenomena have been observed individually in other experimental realizations, either by the mechanical impact of a drop on a solid obstacle [42] or by the impact of a shock wave on a thin liquid film [117]. In these studies, Rayleigh-Plateau and Rayleigh-Taylor instabilities have been identified as the cause of the fragmentation. The Rayleigh-Taylor instability is driven by virtue of an acceleration at an interface between two media of different density [68]. The acceleration in our system is caused by the laser impact, localized both in time and space, and by capillary restoring forces. The latter also minimize the surface energy of the system leading to the RayleighPlateau instability.

The problem in the present work deviates in two important aspects from the aforementioned studies. First, the laser impact allows to clearly separate the timescale of the drop acceleration and the subsequent fluid-dynamic response as we have seen in $\S 4$ and $\S 5$. By contrast, during the mechanical impact of a drop on a solid both deceleration and deformation take place on the same timescale. Second, the deformation of the drop into a thin sheet and the destabilization of this sheet are ultimately the result of the very same initial acceleration. This aspect sets our experiments apart from the fragmentation of a liquid film due to the impact of a shock wave. In this experiment, the film is created manually and prior to the impact.

A key application that requires understanding of drop fragmentation by laser-pulse impact is found in laser-produced plasma light sources for extreme ultraviolet (EUV) nanolithography. In these sources, a dual-pulse sequence results in the emission of EUV light from a tin plasma [15-17]: a first laser pulse shapes a liquid tin drop into a thin sheet to create a suitable target for the impact of a second, much more energetic, laser pulse that generates the plasma. Line emission from excited tin ions in the plasma provides the EUV light $[15,18]$. In this process, the spatial distribution of liquid tin during the second pulse is crucial for an efficient EUV light generation. The distribution is directly affected by the fragmentation of the sheet after the first pulse. Since both the drop deformation and fragmentation have their origin in the same acceleration by the first laser impact they are coupled to each other, which 
(a)

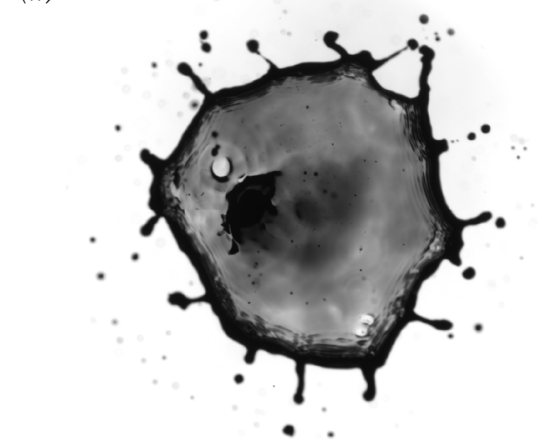

(b)

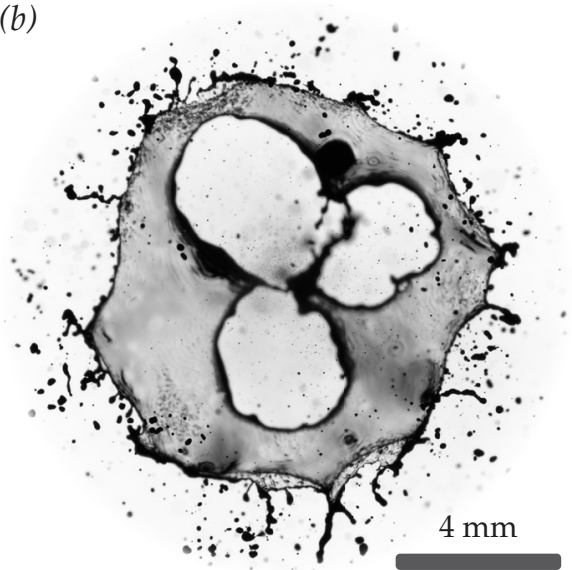

(c)

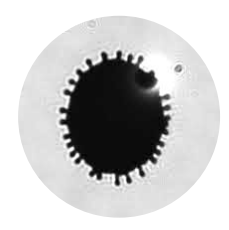

(d)

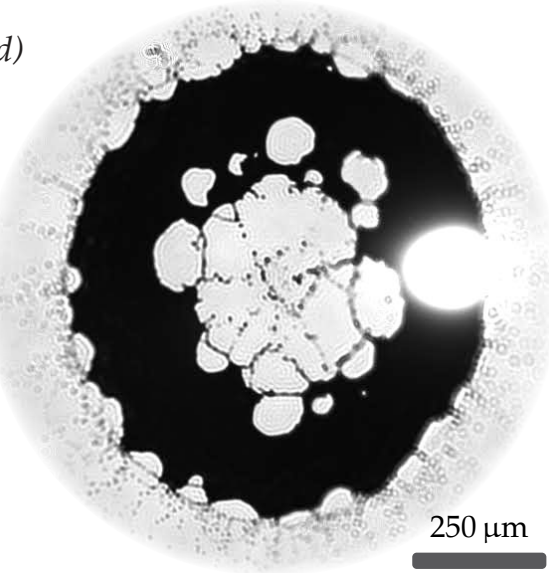

Figure 7.1: Fragmentation of liquid drops consisting of methyl ethyl ketone (MEK) in $(a, b)$ and tin in $(c, d)$ as a result of a laser impact. The drops are accelerated by the laser impact and deform into thin liquid sheets that break by the radial expulsion of ligaments as shown in $(\mathrm{a}, \mathrm{c})$ and by the nucleation and growth of holes on the thin sheet itself $(\mathrm{b}, \mathrm{d})$. The two liquid systems differ in the initial radius $R_{0}=0.9 \mathrm{~mm}$ for MEK and $R_{0}=24 \mu \mathrm{m}$ for tin, and in the laser-matter interaction that induces the drop acceleration: the millimeter-sized MEK drops are accelerated by the local boiling of liquid and the metallic tin drops by an expanding and glowing plasma cloud, which is visible in (c, d) as white spot.

renders the breakup of the liquid an intrinsic part of the laser impact on a drop. The fundamental understanding of the fragmentation is therefore of great importance to improve laser-produced EUV light sources for nanolithography.

Here, we aim to understand in detail how the initial laser impact on the spherical drop causes the fragmentation of the liquid sheet that develops as a late-time response to the very same laser impact. To this end, we study in $\S 7.4$ the breakup process for two different liquid systems, which each have their own unique advantages for the study of fragmentation. On the one 
hand, we use millimeter-sized MEK drops (Fig. 7.1 (a, b)), which expand into semi-transparent sheets that are accessible for high-resolution and high-speed visualization of small details. On the other hand, we use micrometer-sized metal drops (Fig. 7.1 (c, d)), which experience near-perfect axisymmetric impact conditions that do not obscure our view on any fluid-dynamics instability. The details of the liquid systems and experimental setups are described in $\S 7.2$ and $\S 7.3$. Having developed a qualitative understanding of the destabilization mechanism as explained in $\S 7.5$, we aim to quantify the fragmentation by a description in the framework of fluid-dynamics instabilities. First, we adapt an existing model in $\S 7.6$ to describe the characteristic change of the liquid topology as a response to the laser impact: a spherical drop deforms into a liquid sheet that expands radially under the influence of surface tension. With a description of the kinematics at hand, we turn to the breakup at the bordering rim in $\S 7.7$ and the sheet breakup in $\S 7.8$. We will address in our analysis how the laser-matter interaction affects both the deformation of the sheet and an impulsive Rayleigh-Taylor instability. We finally present scaling laws for the characteristic time and wavenumber for both the breakup at the bordering rim and the sheet breakup.

\subsection{Liquid systems}

A laser-induced phase change is a way to move liquids by optical radiation and it allows for large deformations and flow speeds to be reached. The laser radiation must be strong enough to supply the energy required for the phase change, which can be a vaporization or the generation of a plasma. We make use of pulsed lasers to accelerate a free-falling liquid drop by a laser-induced phase change on a timescale that is much shorter than the subsequent fluiddynamic response of the drop. The observed deformation of the drop upon laser impact is then determined to a large extent by the Weber number as explained in $\S 4$ to $\S 6$,

$$
\mathrm{We}=\frac{\rho R_{0} U^{2}}{\gamma}
$$

where $\rho$ is the liquid density, $R_{0}$ the initial drop radius, $\gamma$ the surface tension, and $U$ the center-of-mass velocity of the drop as a result of the laser impact. The Weber number has also been identified as an important parameter in the fragmentation of liquid films and jets [50, 52, 56, 117]. To understand now the drop fragmentation upon laser impact, we make use of two liquid systems that allow us to explore a large range in We. In this section, we introduce both system together with their characteristics that are of importance for the study of fragmentation. 
In $\S 4$ we focused on the question how a laser pulse can induce fluid motion in the first liquid system under consideration here: a liquid-dye solution. We showed that the key driving mechanism for the observed acceleration is the local boiling of the drop that is induced by the absorption of laser energy in a superficial layer. The thickness $\delta$ of this layer is determined by the amount of dye dissolved in the liquid and the absorption coefficient of the dye at the laser wavelength $\lambda_{\mathrm{L}}$. The laser-dye combination and the drop size can then be chosen such that only little mass of the drop is affected by the laser impact, that is $\delta / R_{0} \sim 10^{-2} \ll 1$. The superficial layer $\delta$ of the drop is then ejected at the thermal speed $u$ during the rapid vaporization on a timescale $\tau_{e} \sim 10 \mu \mathrm{s}$. On the same timescale, the resulting recoil pressure $p_{e}$ accelerates the remainder of the drop to the center-of-mass velocity

$$
U \sim \frac{E_{\mathrm{abs}}-E_{\mathrm{th}}}{\rho R_{0}^{3} \Delta H} u,
$$

where $E_{\mathrm{abs}}$ is the energy absorbed by the drop, $E_{\mathrm{th}}$ is the threshold energy that is needed to heat the liquid layer to the boiling point, and $\Delta H$ is the latent heat of vaporization as we explained in $\S 4$. The scaling law (7.2) motivates our choice to use the solvent methyl ethyl ketone (MEK) for the current study: the low value of $\Delta H$ allows to induce large drop velocities for a given laser energy, which translated in view of Eq. (7.1) to a large Weber number range. An alternative liquid is an aqueous solution of Acid-Red-1, which is more convenient to handle experimentally but the range in We is limited due to the large value of $\Delta H$ for water. We characterized both liquid-dye solutions in $\S 3$. In particular, we ensured that $\gamma$ is not affected by the dye, which is crucial for the current study as capillary restoring forces affect both the drop deformation and fragmentation.

The key difference of the second system is found in the driving mechanism of the fluid dynamics: a metal drop is propelled by an expanding plasma cloud in the vicinity of the drop surface as already discussed in $\S 5$. We briefly introduce the concept of this propulsion mechanism, since will see an important influence of the plasma cloud on the expansion rate of the deforming drop in $\S 7.6$. The drop is placed in the focus point of a laser beam where the local fluence exceeds the limit for plasma generation. The intrinsic extinction coefficient of the metal [99] prevents the laser light to penetrate the drop by more than a few nanometer. As soon as a plasma is generated within a fraction of the laser-pulse duration $\tau_{p}=10 \mathrm{~ns}$, inverse-bremsstrahlung absorption strongly decreases the initially high reflectivity of the metallic surface to negligible values. Any further laser radiation is then absorbed by the plasma cloud that exerts a pressure $p_{e}$ on the drop surface accelerating the drop as a whole. The timescale of the drop acceleration is set by the 
plasma dynamics, which is of the same order as the laser-pulse duration: $\tau_{e} \sim \tau_{p}=10 \mathrm{~ns}$. Therefore, the description of the drop propulsion by a short recoil pressure $p_{e}$ is analogous to the vapor-driven drop in the first system. The center-of-mass velocity $U$ for the metal drop follows a similar scaling law with respect to the absorbed energy, that is $U \sim\left(E_{\mathrm{abs}}-E_{\mathrm{th}}\right)^{0.59}$, where $E_{\mathrm{abs}}, E_{\mathrm{th}}$, and the exponent now have their origin in the plasma dynamics, which we explained in $\S 5$. We either use pure liquid tin $(99.995 \%$ purity by Goodfellow), which is motivated by the industrial application in EUV light sources, or an eutectic indium-tin alloy (50In-50Sn, 99.9\% purity by Indium Corporation) with a conveniently low melting point. Since both liquids are almost equivalent in terms of atomic mass, density, and surface tension we can use them interchangeably in the context of this work and refer to them as the tin system.

Characteristic to both systems is the clear separation of timescales: the laser-matter interaction takes place on a nanosecond timescale set by $\tau_{p}$ and accelerates the drop on the timescale $\tau_{e}$. The drop propulsion is accompanied by a lateral expansion that occurs on the inertia timescale $\tau_{i}=R_{0} / U$ and is

Table 7.1: Characteristics of the two liquid systems used in the present work. MEK refers to a solution of methyl ethyl ketone and the dye Oil-Red-O enclosed in a nitrogen environment at ambient temperature (for details on the dye manufacturer see §3). The second system consists of liquid tin at an elevated temperature in a vacuum environment (manufacturer of the liquids given in the text).

\begin{tabular}{llcc}
\hline parameter & unit & MEK & Tin \\
\hline$T$ & liquid temperature $\left({ }^{\circ} \mathrm{C}\right)$ & 20 & 260 \\
$\rho$ & liquid density $\left(\mathrm{kg} \mathrm{m}^{-3}\right)$ & 805 & 6968 \\
$\nu$ & liquid viscosity $\left(\mathrm{m}^{2} \mathrm{~s}^{-1}\right)$ & $0.53 \times 10^{-6}$ & $0.27 \times 10^{-6}$ \\
$\gamma$ & surface tension $\left(\mathrm{N} \mathrm{m}^{-1}\right)$ & 0.025 & 0.544 \\
\hline$R_{0}$ & initial drop radius (m) & $0.9 \times 10^{-3}$ & $24 \times 10^{-6}$ \\
$\tau_{c}$ & capillary timescale (s) & $5 \times 10^{-3}$ & $13 \times 10^{-6}$ \\
$\tau_{i}$ & inertial timescale (s) & $\sim 10^{-4}$ & $\sim 10^{-6}$ \\
$\tau_{e}$ & propulsion timescale (s) & $10 \times 10^{-6}$ & $10 \times 10^{-9}$ \\
$\tau_{p}$ & laser duration (FWHM) (s) & $5 \times 10^{-9}$ & $10 \times 10^{-9}$ \\
$\lambda_{\mathrm{L}}$ & laser wavelength (nm) & 532 & 1064 \\
- & propulsion mechanism & vapor-driven & plasma-driven \\
\hline We & Weber number range & $90-2000$ & $5-18500$ \\
$\operatorname{Re}$ & Reynolds number range & $3000-14000$ & $400-22000$ \\
Oh & Ohnesorge number & $\ll 1$ & $\ll 1$ \\
\hline
\end{tabular}


eventually slowed down by surface tension on the capillary timescale $\tau_{c}=$ $\left(\rho R_{0}^{3} / \gamma\right)^{1 / 2}$. The clear separation of timescales then reads

$$
\tau_{e} \ll \tau_{i}<\tau_{c},
$$

all of which are listed in Table 7.1 for an overview on the two systems. In summary, the combination of the MEK and tin system allows us to study the fragmentation upon laser impact for a large range of Weber numbers. We neglect viscous effects as the Ohnesorge number $\mathrm{Oh}=\sqrt{\mathrm{We}} / \mathrm{Re} \ll 1$, where the Reynolds number $\operatorname{Re}=U R_{0} / v$ is larger than We, see Table 7.1. The fragmentation then shows the same features and chronology for both systems as we will see in $\S 7.4$, while each setup offers advantages for our analysis. On the one hand, the millimeter-sized drops in the MEK system expand into semi-transparent sheets that are accessible for a high-resolution visualization. High-speed recordings of the fragmentation are only possible for the MEK system, since the timescale $\tau_{c}$ needs to be long enough to be resolved by the frame rate of the camera. On the other hand, the micrometer-sized tin drops allow to achieve much higher Weber numbers under near-perfect impact conditions that are free of azimuthal modulations in the propulsion mechanism as will be explained in the next section.

\subsection{Experimental setups}

We impact a free-falling drop with a carefully-timed laser pulse to propel and deform the drop into a thin sheet that finally breaks. The two liquid systems at vastly different length scale require two individual experimental setups, which are explained in more detail in $\S 3$ for MEK and water drops, and in $\S 5$ for the tin drops. Both setups rely on the same concept, which is sketched in Fig. 7.2, but differ in the details. Here, we only briefly introduce the experimental setups and comment on important differences between them.

\subsubsection{Key concept of the experiment}

In both setups, a drop falls down towards the laser-impact position while it relaxes to a spherical shape with radius $R_{0}$, see Fig. 7.2. On its way, the drop passes through a horizontal light sheet that generates a precise reference trigger for the main laser to impact the drop, the energy meter to acquire the laser-pulse energy $E_{\mathrm{L}}$, and two cameras and a beam profiler for visualization. The complete arrangement of the trigger laser, photodiode, and equipment for the drop generation can be moved to align the drop in $\vec{e}_{y}$ - and $\vec{e}_{z}$-direction. A delay between the reference trigger and the timing of the laser pulse allows 
(a)

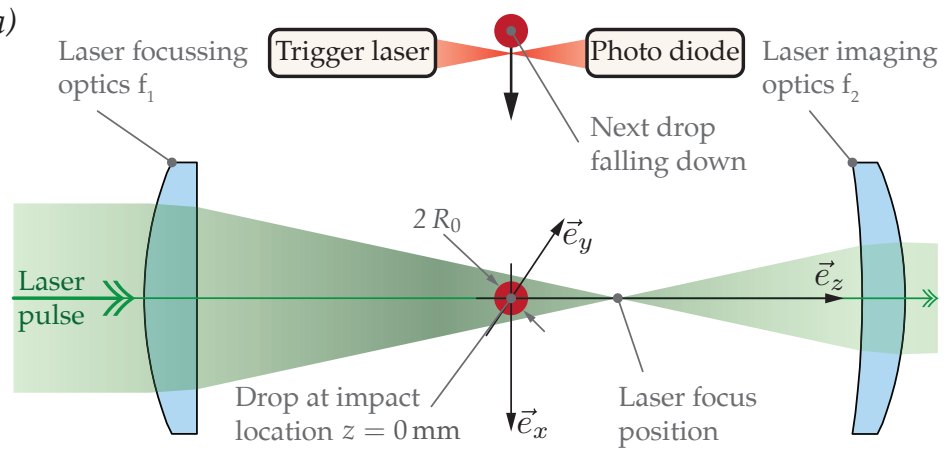

(b)

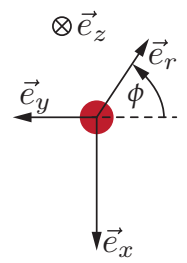

Figure 7.2: (a) Concept of a drop-impact experiments illustrating the moment of laser impact at $t=0 \mathrm{~s}$ in a side-view. The laser pulse is focused by a lens with an effective focal length $f_{1}$, hits the drop, and is captured by an imaging lens $f_{2}$ to redirect the beam onto a charge-coupled device (CCD) for visualization. The drop at the impact location defines the origin of our coordinate system, which is sketched in (b) for the back-view (i.e. in $\vec{e}_{z}$-direction). The experiment is repeated as soon as the next drop falls down in $\vec{e}_{x}$-direction, which is illustrated by the drop at the top of the sketch.

to position the drop in $\vec{e}_{x}$-direction relative to the laser beam. As the drop reaches the impact position, a high-energetic pulse of light enters from the left through a focusing lens $\mathrm{f}_{1}$, hits the drop at $x=y=z=0 \mathrm{~m}$, and exits to the right through the imaging lens $\mathrm{f}_{2}$.

The impact conditions for the drop can be visualized with the laser light captured by lens $\mathrm{f}_{2}$ as will be explained in $\S 7.3 .4$. The subsequent response of the drop to the laser impact is visualized in two orthogonal views: the sideview is aligned to the $\vec{e}_{y}$-axis, whereas the back-view records images in the direction of the laser-beam propagation $\vec{e}_{z}$, see Fig. 7.2(b). We obtain stroboscopic videos by performing a single impact experiment per video frame while changing the time delay between the laser impact and the pulsed light source that illuminates the scene of interest. Our image analysis then obtains the drop position in all three coordinate directions as a function of time, which is used to calculate the velocity $U$ in $\vec{e}_{z}$-direction. The sheet radius $R$ is determined as the equivalent radius of a circle with the same area as the shape detected in the $x y$-plane. Experiments that suffer considerably from a laser-to-drop misalignment or variations in the laser energy are excluded in our analysis: the velocity $U$ is constant for $t>\tau_{e}$ and leads to an accurate prediction for the center-of-mass position in each video frame. We then filter typically the worst $10 \%$ of experimental realizations to study the fragmentation process at well-defined control parameters, which is important to obtain unbiased statistics as we will see in $\S 7.8$. The technical equipment that is used for the MEK and tin experiments differ, as explained in the following. 


\subsubsection{Experimental MEK setup}

Drops with an initial radius $R_{0}=0.9 \mathrm{~mm}$ are generated by the quasi-static pinch-off from a capillary tube. A filter with a pore size of $200 \mathrm{~nm}$ is placed at the inlet to the capillary tube to prevent solid contaminations to enter the drop that might be introduced when the dye is dissolved into the liquid. The experiment is performed in a nitrogen environment, since MEK easily forms explosive mixtures with ambient air. The excitation source is a Nd:YAG laser system at its first harmonic wavelength $\lambda_{\mathrm{L}}=532 \mathrm{~nm}$ emitting pulses with a duration of $\tau_{p}=5 \mathrm{~ns}$ full width at half maximum (FWHM). To improve the uniformity of the incident radiation on the drop, we use a beam-shaping and near-field imaging technique. However, for a full description of the beam preparation, drop-impact chamber, and a study on the stability of the experimental control parameters we refer the reader to $\S 3$.

To resolve all relevant time and length scales a stroboscopic imaging technique with nanosecond exposure times (High Efficiency Diffuser by LaVision pumped by a Nd:YAG laser to remove the coherence of the laser light by incoherent laser-induced fluorescence light illumination (iLIF) [67]) in combination with high-resolution cameras (pco.4000 by PCO AG and Bigeye G-1100B Cool by Allied Vision Technologies, each with $4008 \times 2672 \sim 10^{7}$ pixels) is used. As the drop is propelled along the $\vec{e}_{z}$-axis we move the back-view camera with a motorized stage to keep the deforming drop in the depth of field for each video frame. This procedure is necessary to resolve small details on the semi-transparent MEK sheets as we will see in $\S 7.4$. Since the fragmentation takes place on the timescale $\tau_{c} \approx 5 \mathrm{~ms}$ in the MEK system, we can record continuous videos of the fragmentation following typical criteria for high-speed imaging [66]. Our high-speed system consists of two continuous light sources (LS-M352A metal halide light source by SUMITA Optical Glass and a MAX303 xenon light source by Asahi Spectra) and high-speed cameras (FASTCAM SA-X2 and SA1.1 by Photron) in a backlit configuration. We record high-speed videos at a maximum resolution of $1024 \times 1024 \sim 10^{6}$ pixels with a frame rate of $f_{\text {cam }}=10000$ frames per second. The advantage of the high-speed recordings in the current study is the ability to follow individual breakup events, which are not necessarily reproducible due to the stochastic nature of the fragmentation.

\subsubsection{Experimental tin setup}

Drops of liquid tin are dispensed from a pressure-driven drop generator with an initial radius of $R_{0}=24 \mu \mathrm{m}$ at a temperature of $260^{\circ} \mathrm{C}$. The laser is a $\mathrm{Nd}$ :YAG system emitting pulses of $\tau_{p}=10 \mathrm{~ns}$ (FWHM) at the fundamental wavelength $\lambda_{\mathrm{L}}=1064 \mathrm{~nm}$. Since the laser beam is focussed to generate a 
plasma at the drop surface, the experiment is placed in a vacuum vessel, typically at $10^{-7} \mathrm{mbar}$, to prevent an optical breakdown before the laser pulse hits the drop. The vacuum environment also prevents an oxidization of the metallic surface of the drops.

For stroboscopic visualization of the expanding tin drops we use a shadowgraph imaging systems based on pulsed lasers and CCD cameras (Manta G-145B NIR by Allied Vision Technologies) coupled to long-distance microscopes (Infinity K2 DistaMax with CF-1/B objective and NTX tube (2x)). The back-view camera in the tin system is aligned to the $\vec{e}_{z}$-axis under a weak parallax angle that leads to a tilt of the image plane relative to the deforming sheets. This arrangement leads to an apparent elliptical shape of the tin sheets in the back-view images, which we correct in our image analysis when quantitative data is obtained. In early experiments, pulsed laser diodes (PLD) (about 15-ns pulse length) acted as backlight sources for illumination. In more recent experiments, the PLDs were replaced by a pulsed dye-laser pumped by the second harmonic wavelength of a Nd:YAG laser, emitting an approximately 5 -ns-long pulse of 560-nm light with a spectral width of $4 \mathrm{~nm}$. Such illumination enables imaging of small features of the expanding tin sheets by reducing detrimental effects due to temporal coherence such as speckle.

\subsubsection{Beam profile}

The interaction of the laser beam with the liquid gives rise to the pressure $p_{e}$ on the drop surface that drives the fluid-dynamic response. To get detailed information on the driving force, we measure the laser beam profile with a CCD in absence of the drop before an actual impact experiment. In both systems, the imaging lens $f_{2}$ (Fig. 7.2) can be used to capture the incident radiation, i.e. the fluence $F(x, y, z=0)$ in the impact plane spanned by $\vec{e}_{x}$ and $\vec{e}_{y}$. The CCD only records the relative fluence $f(x, y, z=0)$ that is translated to absolute terms

$$
\begin{aligned}
F(x, y, z=0) & =F_{0} f(x, y, z=0) \text { with } \\
F_{0} & =E_{\mathrm{L}}\left(\int_{A} f(x, y, z=0) \mathrm{d} A\right)^{-1},
\end{aligned}
$$

where we measure $E_{\mathrm{L}}$ with an energy meter and chose the area of integration $A$ large enough to enclose the complete beam.

For the MEK system, the driving pressure is caused by the vapor recoil that is a direct result of the laser-matter interaction at the drop surface. As such, the pressure preserves any spatial fluctuation present in $F(x, y, z=0)$ at the drop location. To determine the drop position and size in relation to the 
laser beam, we use the CCD image data during each realization of an impact experiment, see Fig. 7.3 (a). We then compute the fluence $F_{\text {abs }}$ that is absorbed by the drop as shown in Fig. 7.3(b). The pressure $p_{e}$ on the drop surface is now directly proportional to $F_{\mathrm{abs}}$, from Eq. (7.2) we obtain

$$
p_{e}(r, \phi) \sim \frac{F_{\mathrm{abs}}(r, \phi)-F_{\mathrm{th}}}{\Delta H} \frac{u}{\tau_{e}} .
$$

Combined with the measurement in Fig. 7.3(d) we recognize that the MEK drops are subject to a driving force that varies in azimuthal direction $\phi$ by about $\pm 10 \%$. Since $f$ is found to be independent of $E_{\mathrm{L}}$, the azimuthal variation in the MEK experiments is then also independent of $E_{\mathrm{L}}$ and fixed in the Galilean reference frame of the laboratory for all our experiments.

By contrast, the tin drops experience a near-perfect axisymmetric driving force. A lens with a focal length of a $f_{1}=1000 \mathrm{~mm}$ focuses the laser beam to a circular Gaussian spot, where the drop is then placed. Such an optical arrangement leads to a diffraction-limited spot diameter of approximately $100 \mu \mathrm{m}$, which can be estimated by the extent $\omega_{0}$ of an Airy disk [65] that scales as $\omega_{0} \sim \lambda_{\mathrm{L}} \mathrm{f}_{1} / d_{0}$ with $d_{0}$ as the laser-beam diameter before lens $\mathrm{f}_{1}$. However, this optical arrangement dictates a length scale of a few micrometer for the tin system, that is $R_{0}=24 \mu \mathrm{m}<\lambda_{\mathrm{L}} \mathrm{f}_{1} / d_{0}$. The advantage of the focussing condition is a homogenous laser radiation at the impact location, see Fig. $7.3(\mathrm{e}, \mathrm{f})$. In addition to the homogenous laser radiation, any spatial fluctuation comparable to $R_{0}$ is removed by the intermediate step of the plasma generation during the drop acceleration: the tin drop is not subject to the direct laser radiation but shielded by its own plasma cloud as explained in $\S 7.2$. As a consequence, the fluid dynamics obey a high degree of rotational symmetry as we will see in $\S 7.4$. 


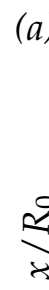

(c)

$$
\begin{aligned}
& \text { a) }-1 \\
& -0.5 \\
& \frac{2}{y} \quad 0 \\
& 0.5
\end{aligned}
$$
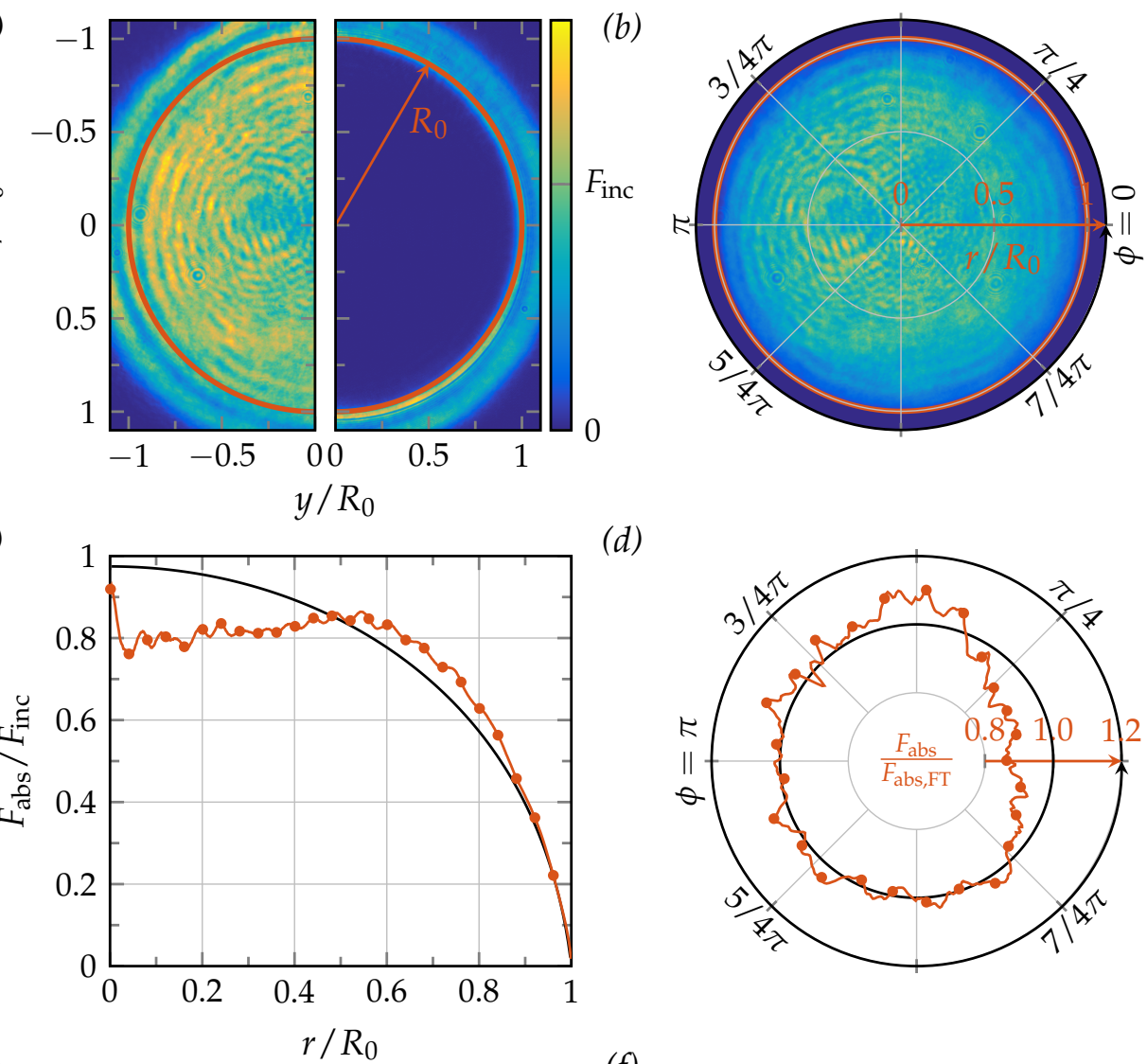

(e)

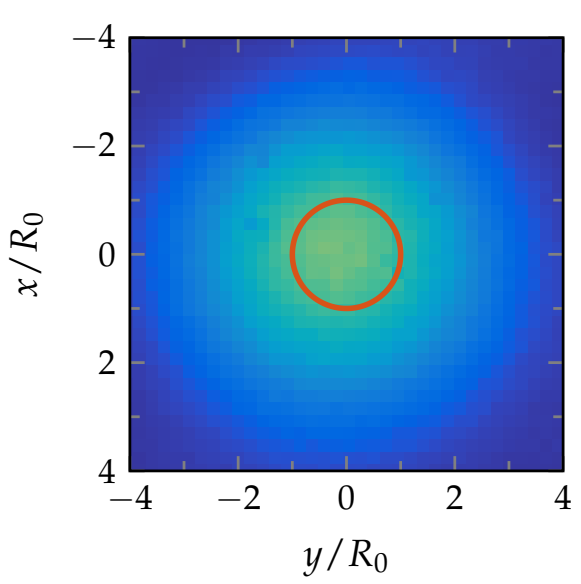

(d)

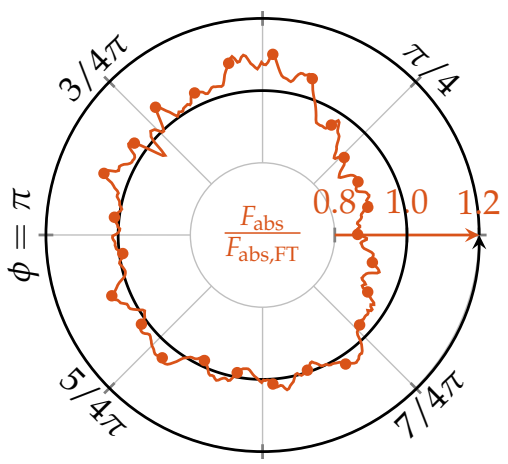

(f)

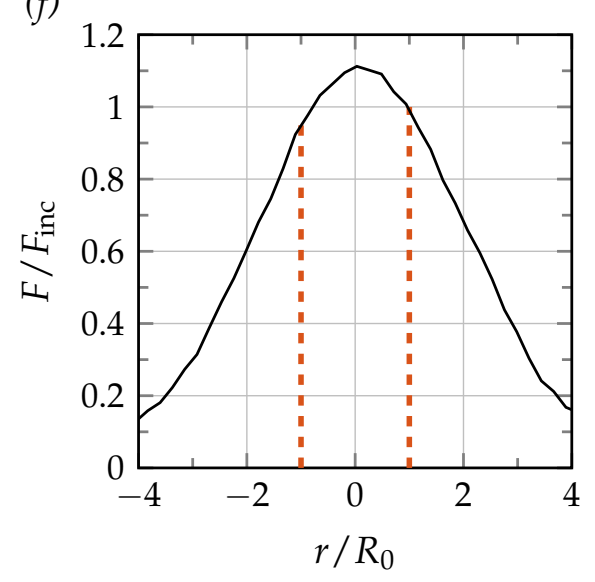

Figure 7.3: (Continued on the following page.) 
Figure 7.3: (Figure is found on the previous page.) (a) A planar laser-beam profile in the MEK system as recorded in the absence of the drop for $y / R_{0} \leq 0$ and recorded during an actual experiment with the drop for $y / R_{0} \geq 0$. The latter yields the drop radius $R_{0}$ and position in the beam profile as indicated by the solid line ( -$)$. The quantity $F_{\text {inc }}$ is the average fluence incident on the drop. (b) The energy per unit area absorbed by the drop, that is the fluence $F_{\text {abs }}$ taking losses due to Fresnel reflection [65] at the liquid-air interface into account. Based on approximately 100 recordings of such a spatial distribution a mean profile ( -- ) in radial (c) and azimuthal direction (d) is determined. The solid line $(-)$ represents the predicted result for a perfect flat-top beam profile (indicated by $F_{\mathrm{abs}, \mathrm{FT}}$ in (d)) (e) A planar laser-beam profile measured for the tin system and the drop marked in the center $(-)$ at its expected position. The same colorbar as in (a) is used but the profile exhibits less spatial variations at the drop position, which explains the absence of yellow hotspots in the image. (f) The gaussian beam profile in radial direction $r$ recovered from (e).

\subsection{Chronology}

The MEK experiment in Fig. 7.4 illustrates the chronology of the drop fragmentation, which starts with the laser impact and ends with a structure of ligaments that subsequently break into smaller drops. First, the drop accelerates on the timescale $\tau_{e} \sim 10 \mu \mathrm{s}$ and starts to move forward in $\vec{e}_{z}$-direction at the velocity $U$ while it expands in radial direction $r$. At $t=0.27 \mathrm{~ms}$ the drop already resembles a thin sheet. This time corresponds to the inertia timescale $\tau_{i}=R_{0} / U=\tau_{c} \mathrm{We}^{-1 / 2}=0.28 \mathrm{~ms}$ of that particular experiment. The liquid is semi-transparent in our visualization, which allows us to observe a thinner outer region of the sheet that is bordered by a thicker and hence darker rim. Likewise, the center of the sheet is still thick compared to the outer region. As the sheet radius $R(t)$ further expands with time the sheet thickness decreases as shown by the growing gray areas on the sheets at $t=0.54$ to $1.7 \mathrm{~ms}$. The variation of gray values on the sheet in Fig. 7.4 shows that the sheet thickness is a function of time and space, that is $h(r, t)$. We notice modulations of the sheet thickness in azimuthal direction $\phi$, but the sheet in the back-view images preserves a near-circular shape during the expansion, i.e. an axisymmetric evolution about $\vec{e}_{z}$.

The fragmentation of the drop is observed as a continuous evolution that accompanies the deformation of the drop into a thin sheet and the radial expansion of the sheet thereafter. Two types of breakup can be identified in Fig. 7.4. First, the breakup of the bordering rim: corrugations are visible on the rim at $t=0.27 \mathrm{~ms}$, but they are still small in relation to the sheet radius $R$. The corrugations grow over time until ligaments are expelled radially outward, which is visible for the first time in Fig. 7.4 at $t=0.54 \mathrm{~ms}\left(t / \tau_{c}=0.1\right)$. The 
ligaments break to from stable drops that continue to move radially outward at a constant speed, which is comparable to $\dot{R}$ at the moment of detachment from the sheet. As a result, the sheet at $t=1.1 \mathrm{~ms}$ in Fig. 7.4 is surrounded by a cloud of tiny drops.

The breakup of the thin sheet is the second type of destabilization in our experiments and follows the same principle as observed for the rim: corrugations appear on the liquid body that eventually result in a change of the topology with the liquid mass collected in elongated ligaments. Then, the ligaments break to form stable drops. Only now, the fragmentation is not localized to an one-dimensional structure as for the rim but takes place on the two-dimensional sheet. Corrugations are visible on the sheet at $t=$ $1.1 \mathrm{~ms}$ in Fig. 7.4, where a pointer at the top highlights a patch with large spatial frequency components. We observe such disturbances on the sheet to precede any hole nucleation, including multiple holes piercing a single patch of corrugations. Figure 7.4 shows two cases where a single hole nucleates in a corrugated region: at $1.1 \mathrm{~ms}$ the lower pointer marks a hole shortly after it pierced the sheet close to the outer rim and at $1.7 \mathrm{~ms}$ the same process is captured in the center of the sheet. Once a hole nucleates on the sheet it continues to grow, thereby collecting the surrounding liquid mass of the sheet in a rim. The last frame at $t=2.5 \mathrm{~ms}$ in Fig. 7.4 shows the result of multiple holes growing and eventually merging over time: the liquid of the sheet is finally collected in a two-dimensional structure of ligaments that breaks into stable drops.

A comparison of the MEK and tin system in terms of the drop deformation and fragmentation is shown in Fig. 7.5. The first row shows the destabilization of the bordering rim for an otherwise intact sheet at low Weber number. The rim cannot be observed directly for the tin sheets, since they appear opaque in the visualization due to the large extinction coefficient of visible light in tin [99]. Both systems show the formation of ligaments and the detachment of tiny drops once these ligaments break. We refer to this fragmentation mechanism as rim breakup. The second and third row of Fig. 7.5 show how the sheets break by the nucleation of holes at two distinct regions: either close to the rim or in the center of the sheets. The thinner neck region at $r / R \sim 1$ seems to promote the breakup by hole nucleation, which we refer to as neck breakup. We observe a second location for hole nucleation at $r / R<0.5$, referred to as center breakup.

The neck breakup is observed before the center breakup and may repeat itself several times during the sheet expansion. Holes pierce the sheet in the neck region and continue to grow. Once the holes have reached the outer rim of the sheet, the rim is basically detached from the sheet and breaks up in tiny drops (see Fig. 7.5 (b) and (e)). The liquid mass collected by the holes 


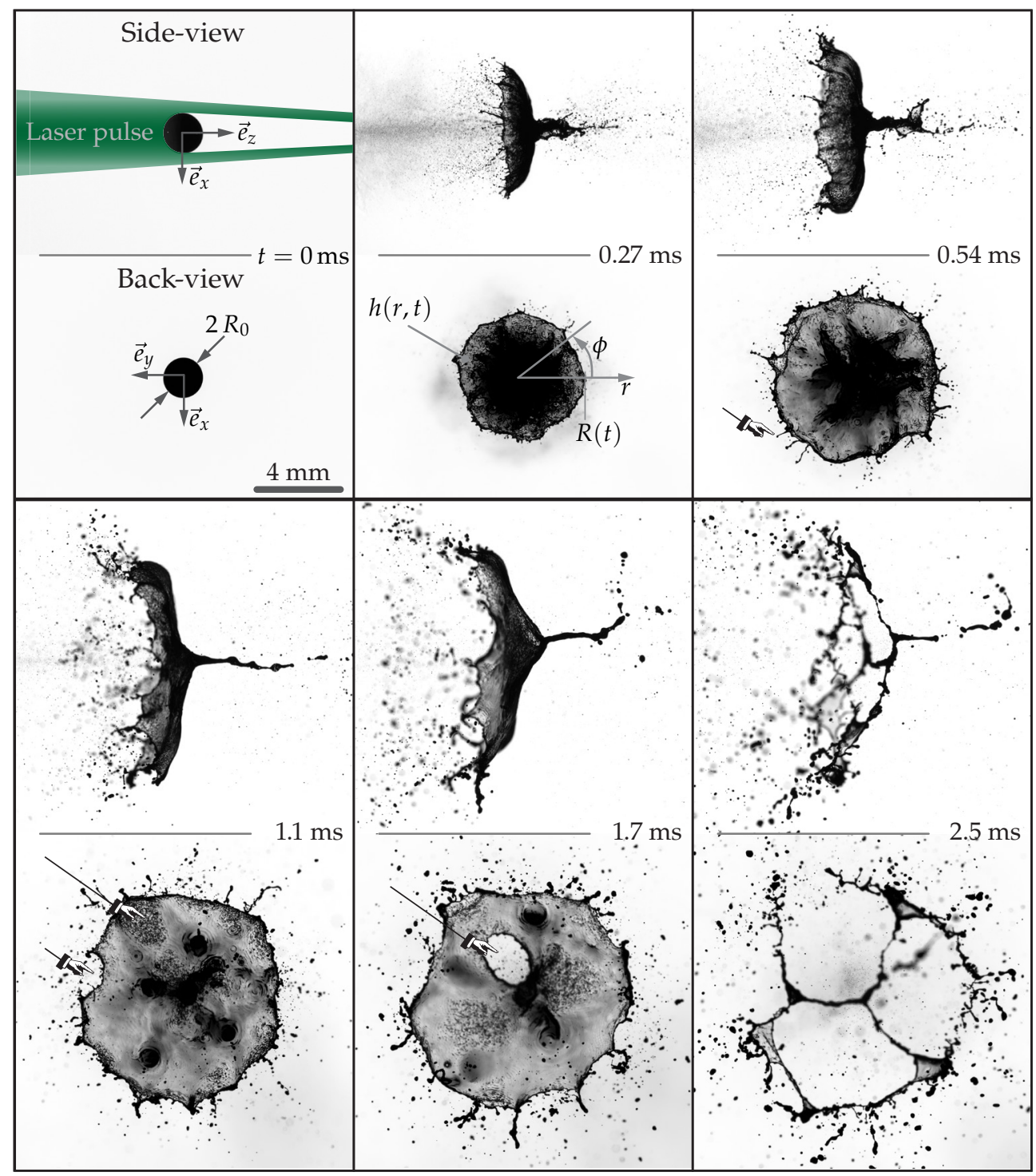

Figure 7.4: Sequence of events following the impact of laser pulse on free-falling drops in the MEK system at We $=330\left(\tau_{c}=5 \mathrm{~ms}\right)$. Images are recorded in stroboscopic fashion in a side- and back-view, where the side-view images are shown in a comoving frame with the propulsion speed $U$ subtracted. At the inertial timescale $\tau_{i}=\tau_{c} \mathrm{We}^{-1 / 2}=0.27 \mathrm{~ms}$, the semi-transparent liquid allows to recognize a thin sheet of radius $R(t)$ and non-uniform thickness $h(r, t)$ that is bordered by a rim. The pointers in the subsequent three pictures mark the onset of fragmentation during the sheet expansion: radial expulsion of ligaments at $t=0.54 \mathrm{~ms}$, corrugated areas and a hole piercing the sheet close to the rim a $t=1.1 \mathrm{~ms}$, and hole nucleation close the center of the sheet at $t=1.7 \mathrm{~ms}$. A final web of ligaments is shown for $t=2.5 \mathrm{~ms}$. 
during their growth forms a new and corrugated rim. The observation of the neck breakup requires a high spatial and temporal resolution. The process is localized in space to a region close to $r / R \sim 1$ and leaves no other trace behind than a corrugated rim and tiny drops. The detached drops contribute to the cloud of drops surrounding the sheet that we already observed for the rim breakup. For example, Fig. 7.1 (c) is shown as an example for the center breakup but the neck breakup already took place (which is why the image is taken at a later time $t$ and the sheet appears larger than the one from the same experiment in (b)). By contrast, the growth of holes during the center breakup is much easier to observe experimentally, which is why we will base our quantitative analysis in $\S 7.8$ mostly on data obtained for the center breakup.

The opaque tin sheets prevent a further comparison of the two systems in terms of the corrugations that are visible for MEK in Fig. 7.5 (b, c). However, both systems show identical, qualitative features for the fragmentation. A difference between the two systems is found in the Weber number at which fragmentation is observed. While the rim breakup is observed for both systems at a comparable Weber number (compare Fig. 7.5 (a) and (d)), more than one order of magnitude in We separates the two systems during the sheet breakup in Fig. $7.5(\mathrm{~b}, \mathrm{c})$ and $(\mathrm{e}, \mathrm{f})$. We will see in $\S 7.8$ that this difference is caused by the different driving mechanisms that affect the sheet destabilization.

\subsubsection{Some comments on jetting}

In addition to the sheet evolution, one observes the ejection of mass on the opposite site of the laser impact in form of a liquid crown (notice the thin and hence gray areas at the tip, visible at $t=0.27 \mathrm{~ms}$ in Fig. 7.4). The ejected mass moves at a speed larger than $U$, collapses on the $\vec{e}_{z}$-axis $(t=0.54 \mathrm{~ms})$, and forms a jet that detaches from the sheet and finally breaks up ( $t=1.1$ to $2.5 \mathrm{~ms}$ ). The jetting, or in general the ablation of mass, is also observed in the tin system as shown in Fig. 7.6 $(a, b)$. However, the effect of this early jetting on the sheet

Figure 7.5: (Figure is found on the following page.) Fragmentation regimes for vapordriven MEK drops ( $\mathrm{a}-\mathrm{c}, R_{0}=0.9 \mathrm{~mm}$ ) and plasma-driven tin drops ( $\mathrm{d}-\mathrm{f}, R_{0}=24 \mu \mathrm{m}$ ). In both systems, the fragmentation of the sheets starts at three distinct locations: the bordering rim (first row: $(a, d))$ and the breakup at the neck (second row: $(b, e)$ ) and center of the sheet (third row: $(c, f)$ ). The apparent elliptical shape of the tin sheets is caused by a weak parallax angle of the camera relative to the propulsion in $\vec{e}_{z}$-direction. The white spot in figure (b) and (c) is an artifact of the plasma that propels the tin drop. Both MEK and tin drops show the same features during the fragmentation, which we refer to as rim, neck, and center breakup (see text). 


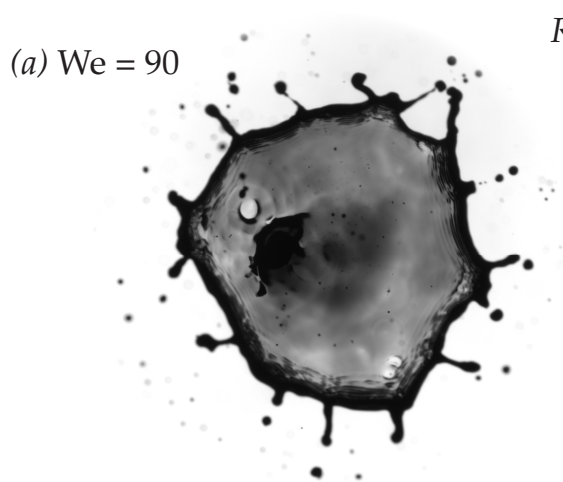

Rim breakup

(d) $\mathrm{We}=260$

(b) $\mathrm{We}=460$

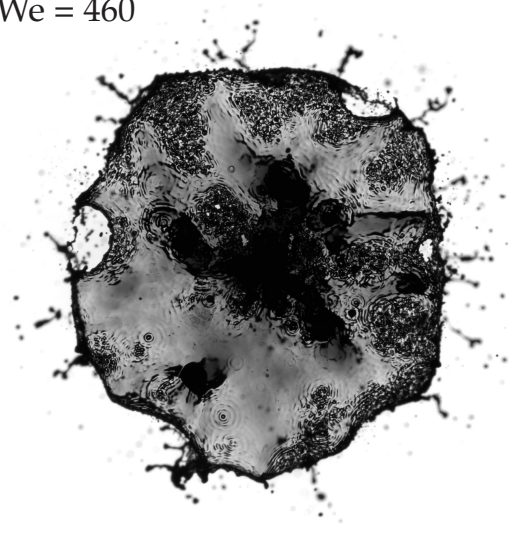

Neck breakup

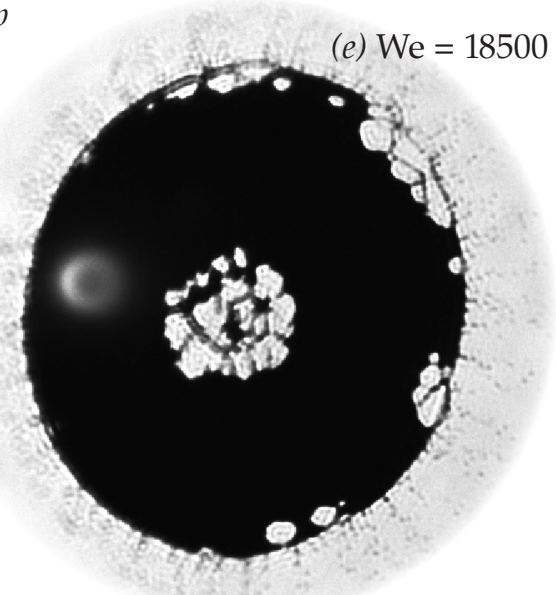

(c) $W e=460 \quad \therefore \quad$ Center breakup

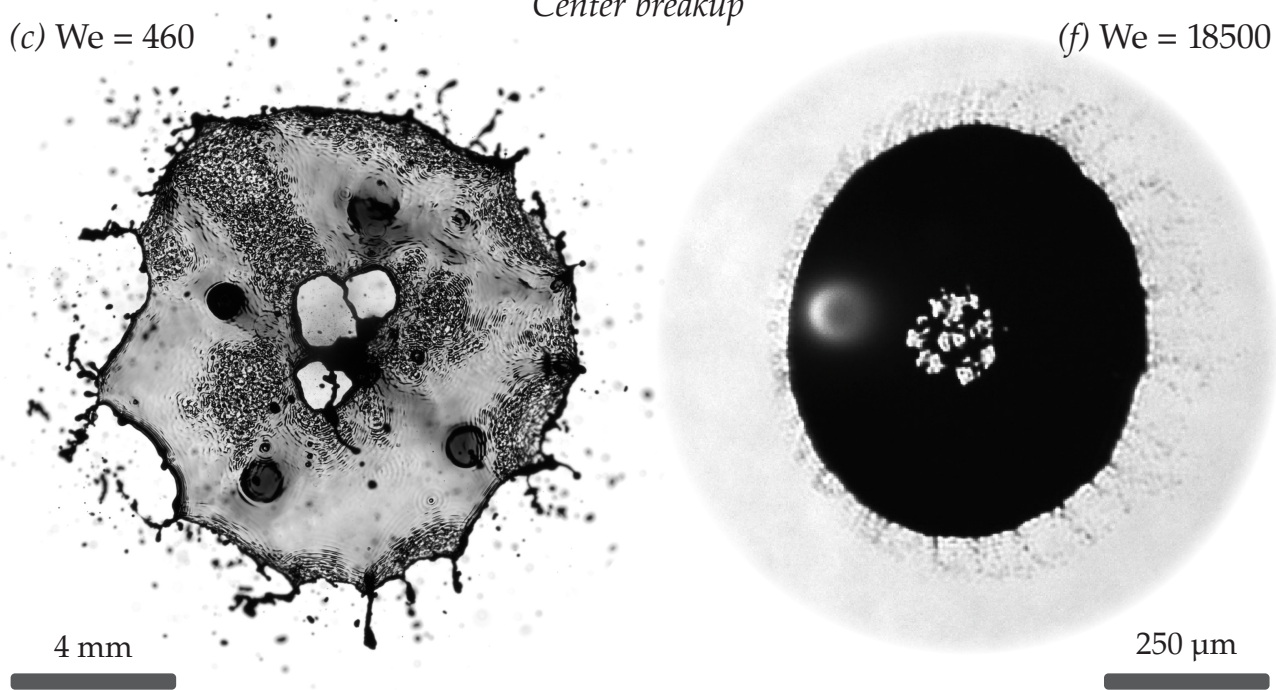

Figure 7.5: (Continued from the previous page.) 
evolution is observed to be small as the ejected mass is small compared to the remainder of the drop. A description of the phenomena is therefore beyond the scope of the present study, while we recognize that it may results in a slightly tampered center region of the sheet when it becomes sufficiently thin as illustrated in Fig. 7.6 (d). Here, we briefly comment on the physical cause that may lead to the jetting phenomena.

The early jetting is not a direct consequence of the pressure pulse driving the expansion: detailed simulations of the drop-shape evolution by a boundary-integral (BI) method, which is capable to reproduce jetting phenomena in principle [119], do not show this feature as we saw in $\S 6$, compare also Fig. 7.6 (c). A possible explanation for the jetting is the occurrence of a cavitation bubble that could give rise to a fast jetting phenomena [33, 36, 120,121]. However, a nucleation of a bubble inside the drop by direct laser radiation is unlikely in our systems. The penetration depth of the laser light for both MEK and tin is limited to fraction of the drop, that is $\delta / R_{0} \ll 1$ as already explained in §7.2. Acoustical effects could however induce a cavitation bubble [121, 122]: pressure transients induced on one side of the drop travel to the opposite side or may even be focus inside the drop. Thermoelastic pressure waves are caused by the rapid heating of the superficial layer of the MEK drops under isochoric conditions $\ddagger[20,123,124]$. The same effect leads to pressure transients in tin drops [92], where in addition the plasma generation is accompanied by shock waves $[125,126]$. We recognize that the compressibility of the liquid and acoustic effects may cause the jetting phenomena. However, the inclusion of these effects in our study on fragmentation is out of scope given the small effect of the jetting on the overall system response, especially the late-time sheet dynamics.

\subsection{Interpretation}

The laser impact on a free-falling liquid drop can be understood as a rapid acceleration on the timescale $\tau_{e}$ that acts on the spherical surface of the drop and causes the subsequent deformation into a thin sheet bordered by a rim. The destabilization of this liquid body is then observed on the timescale $\tau_{i}$ and $\tau_{c}$ as a continuous evolution: corrugations appear and eventually lead to a change of topology such that the liquid mass is collected in elongated ligaments. Then, the ligaments break to form stable drops. This fragmentation process was observed in $\S 7.4$ in two different configurations: the breakup of the bordering

\footnotetext{
¥Thermoelastic pressure transients under isochroic conditions [20] are achieved in the MEK system since $c \tau_{p} / \delta \sim 1$, where $c$ is the speed of sound in the liquid, $\delta$ the layer thickness heated by the laser and $\tau_{p}$ the laser-pulse duration.
} 
(a)

(b)

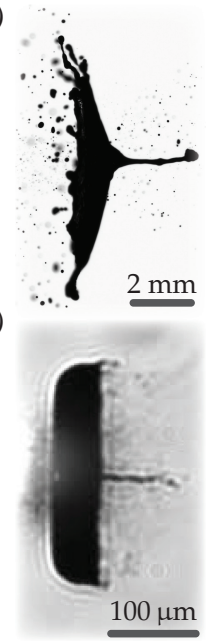

(c)

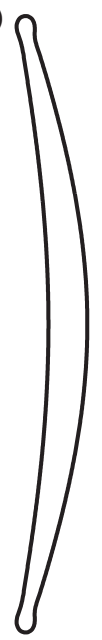

(d)

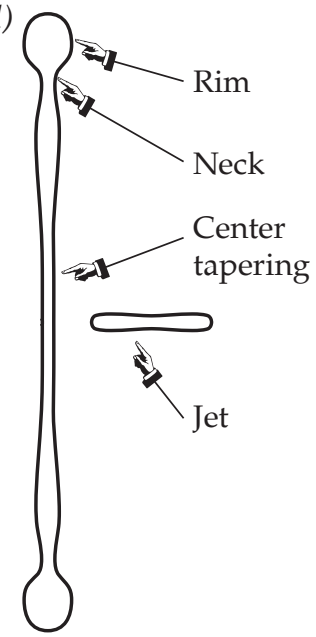

Figure 7.6: $(a, b)$ Side-view images showing the formation of a jet in the center of the drop in the MEK (a) and tin system (c). (c) Sheet contour obtained from a BI simulation illustrating the cross section of the axisymmetric shape for We $=790$ (taken from §6). (d) Sketch of the sheet showing a bordering rim and a tampered neck and center region.

rim, which we referred to as rim breakup, and the hole nucleation on the sheet itself called the sheet breakup. The latter was observed at two different locations on the sheet, which we denoted by neck and center breakup. We now evaluate what the mechanisms are that can possibly cause and amplify corrugations on the deforming liquid drops in our experiments. As we will demonstrate below, the accelerations by the laser impact and capillary restoring forces each induce fluid-dynamic instabilities that lead to the breakup.

The kinematics of the sheet in our experiments are in striking agreement with the impact of a drop on pillar [42]. Also there, the topology of a drop is rapidly changed to an expanding thin sheet that is bordered by a rim. Not surprisingly, we observe the same destabilization and breakup for the rim as described by Villermaux and Bossa [42]. The rim diameter was found to reach an equilibrium value such that the rim is affected by a Rayleigh-Plateau instability, similar to the breakup of an elongated liquid cylinder of constant diameter. In addition, the liquid in the rim is subject to an acceleration that is determined by the expansion and recoil dynamics of the sheet leading to a Rayleigh-Taylor mechanism. Our observations for the nucleation, growth, and merging of holes on a sheet are in agreement with a previous study by Bremond and Villermaux [117]. In their experiment, a thin liquid film is accelerated by the reflection of a traveling shockwave at the liquid film 
itself. The hole nucleation is explained with an impulsive Rayleigh-Taylor instability driven by the strong accelerations perpendicular to the sheet. By contrast, the sheets in our experiments are not subject to a direct acceleration. A possibly candidate in our experiments for a strong acceleration is the initial acceleration of the drop, i.e. an acceleration at a time when the thin sheet has not yet developed. Our analysis then needs to address when both the evolving liquid sheet and a simultaneous Rayleigh-Taylor instability are in such a state for breakup to occur.

Having identified Rayleigh-Plateau and Rayleigh-Taylor instabilities as likely candidate for the destabilization of the liquid sheet, we realize that both the rim and the sheet breakup depend on the sheet kinematics: the radius $R(t)$ determines the acceleration during the rim breakup and the thickness $h(r, t)$ is required to follow the evolution of an instability during the sheet breakup. Consequently, we first seek an analytical description in terms of $R(t)$ and $h(r, t)$ for the deformation and expansion of the drop in $\S 7.6$. With a description of the kinematics at hand, we then turn to the analysis of the breakup itself: in $\S 7.7$ we study the rim breakup and in $\S 7.8$ the breakup of the sheet.

\subsection{Expansion dynamics}

As discussed in $\S 6$, the deformation of a drop by virtue of a laser impact can be described in three stages: an early-time stage $\left(0 \leq t \leq \tau_{e}\right)$ where the spherical drop is accelerated and gains kinetic energy as a result of a recoil pressure $p_{e}$ acting on its surface. Then follows an intermediate stage $\left(\tau_{e}<t \lesssim \tau_{i}\right)$ where the drop deforms into a sheet, and a late-time stage $\left(t \gg \tau_{i}\right)$ where the sheet undergoes a surface-tension limited expansion. In the early-time analytical model the partition of kinetic energy of translation and deformation $E_{\mathrm{k}, \mathrm{d}} / E_{\mathrm{k}, \mathrm{cm}}$ is obtained from the recoil pressure $p_{e}$, which is set by the laser-matter interaction recalling for example expression (7.6). This energy partition is then fed into a late-time model that describes the expansion dynamics in terms of a rescaled Weber number based on the kinetic energy of deformation

$$
\mathrm{We}_{\mathrm{d}}=\frac{E_{\mathrm{k}, \mathrm{d}}}{E_{\mathrm{k}, \mathrm{cm}}} \text { We. }
$$

In $\S 6$ we used a simple late-time model assuming a sheet of uniform thickness. However, in our MEK data we observe a clear radial dependency of the sheet thickness and need to apply a slightly more sophisticated sheet model to accurately describe our expansion data. To this end, we use a model that was previously used to describe the sheet evolution after the drop impact on a pillar [42]. This model described the sheet dynamics away from the 
pillar region and includes a spatial dependency in the description of the sheet thickness $h(r, t) \sim 1 / r$, which has been validated experimentally by Vernay et al. [116]. In this model too, it is assumed that the drop impact itself is decoupled from the subsequent dynamics of the sheet, an assumption that is well justified for our systems in view of the separation of timescales (7.3). Following Villermaux and Bossa [42], we describe the sheet radius $R(t)$ as

$$
\frac{R(t)-R_{0}}{R_{0}}=\sqrt{3 \mathrm{We}_{\mathrm{d}}} \frac{t}{\tau_{c}}\left(1-\frac{\sqrt{3}}{2} \frac{t}{\tau_{c}}\right)^{2} .
$$

The only modification introduced is the rescaling of We in analogy to the approach described in $\S 6$. We choose the rescaling such that the energy partition of the thin sheet at $t=\tau_{i}$, i.e. when the topology of the drop changed to a thin sheet, matches the analytical result from the early-time model, see appendix $\S 7$.A for the details.

The energy partition allows to incorporate different laser-matter interactions that set the recoil pressure $p_{e}$ on the liquid interface during the drop acceleration. Since the relative fluence $f(x, y, z=0)$ in the impact plane is kept constant for all MEK experiments and is directly related to the pressure $p_{e}$ as explained in $\S 7.3$, the energy partition is independent of $E_{\mathrm{L}}$ and can be calculated analytically to $E_{\mathrm{k}, \mathrm{d}} / E_{\mathrm{k}, \mathrm{cm}}=1.8$, which we already used in $\S 6$. In this calculation, we neglect the threshold fluence $F_{\text {th }}$ in expression (7.6) as we operate the experimental setup at $F_{\text {abs }} \gg F_{\text {th }}$, which is necessary to reach large Weber numbers. By contrast, the energy partition for the tin drops is a function of $E_{\mathrm{L}}$, since the plasma dynamics is a function of the incident laser energy, even at constant focusing conditions. We find $E_{\mathrm{k}, \mathrm{d}} / E_{\mathrm{k}, \mathrm{cm}}$ to follow a power law with respect to $E_{\mathrm{L}}$, see Fig. 7.7. A theoretical prediction of the scaling law requires a profound understanding of the plasma dynamics during the laser impact and goes beyond the scope of this study. However, the trend with the laser energy can be explained: a comparison of Figs. $7.7(b-d)$ shows that at lower laser energy the plasma cloud covers a smaller area of the drop surface, which results in an effective focusing of the recoil pressure to a confined region. The more focused a pressure distribution is, the more kinetic energy goes into deforming the drop without translating it as we saw in $\S 6$. This relation then translates to an increase in $E_{\mathrm{k}, \mathrm{d}} / E_{\mathrm{k}, \mathrm{cm}}$ as the laser energy $E_{\mathrm{L}}$ decreases, which is in agreement with our experimental observations.

The comparison of the analytical expression (7.8) to experimental data for both MEK and tin experiments is shown in Fig. 7.8 (a) and (b), respectively. The initial expansion to the maximum radius $R_{\max }$, the moment when $R_{\max }$ is reached at $t_{\max }=2 \tau_{c} / \sqrt{27} \approx 0.38 \tau_{c}$, and the recoil of the sheet due to surface-tension forces are accurately captured by the model. Especially for tin 


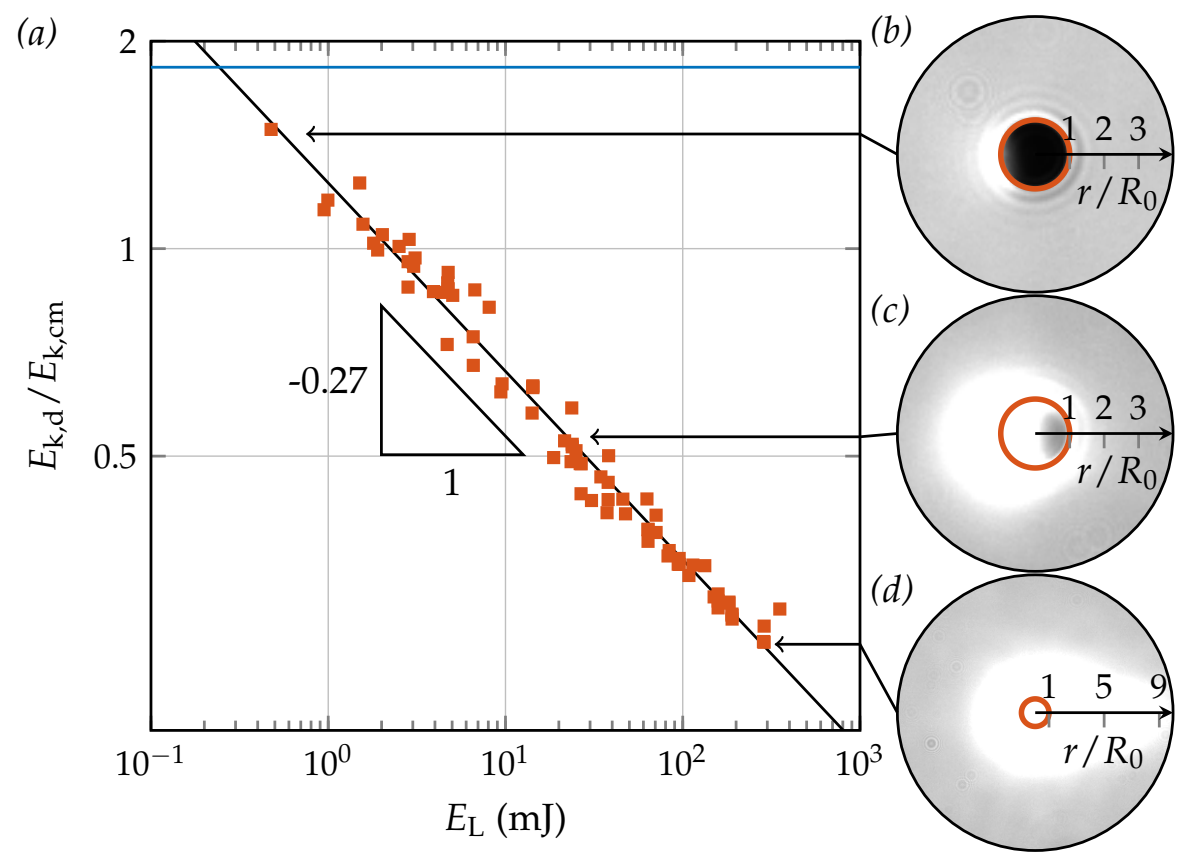

Figure 7.7: Energy partition $E_{\mathrm{k}, \mathrm{d}} / E_{\mathrm{k}, \mathrm{cm}}$ as function of laser energy $E_{\mathrm{L}}$ for MEK (solid line _- ) and tin drops (square markers $\mathrm{-}$ ) in (a). The values for MEK are calculated analytically to $E_{\mathrm{k}, \mathrm{d}} / E_{\mathrm{k}, \mathrm{cm}}=1.8$, independent of $E_{\mathrm{L}}$ (see text). The values for tin (square markers $\bullet$ ) are determined for each experiment by the best fit of expression (7.8) to the experimental curves shown in Fig. 7.8. The solid line (-) is then the power law that follows from a linear regression yielding $E_{\mathrm{k}, \mathrm{d}} / E_{\mathrm{k}, \mathrm{cm}}=0.19\left(E_{\mathrm{L}} / E_{0}\right)^{-0.27}$ with $E_{0}=1 \mathrm{~J}$. The three insets $(\mathrm{b}-\mathrm{d})$ visualize the white plasma clouds that result in the deformation of the tin drops. The initial undeformed tin drops are marked by the red circle (-) in each inset.

the agreement between model and experiment holds over nearly four decades in Weber number (Fig. 7.8 (b)). The model deviates from the experimental results only for cases where the fragmentation severely affects the topology of the sheet. The onset of the sheet breakup is earlier for MEK in comparison to tin as we will see in $\S 7.8$. Consequently, we observe the model here to deviate earlier in Fig. 7.8 (a) for MEK when compared to tin in (b), especially for large Weber numbers that lead to a severe neck breakup as explained in the following.

In the collapsed view of Fig. 7.8 (c) a few cases are highlighted to illustrate how fragmentation affects the comparison between model and experiment. A reference case in absence of any fragmentation is given by We $=5$ ( $)$ that follows the model perfectly. Second, for tin at We $=130\left({ }^{(}\right)$the sheet itself 
(a)

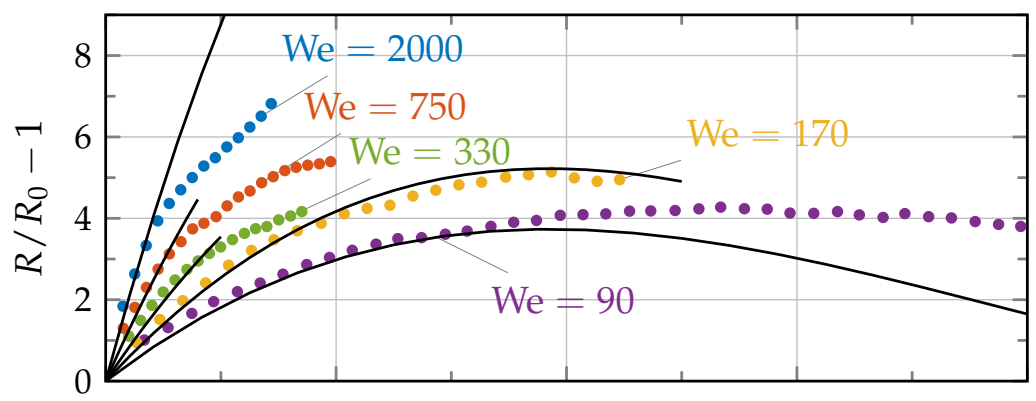

(b)

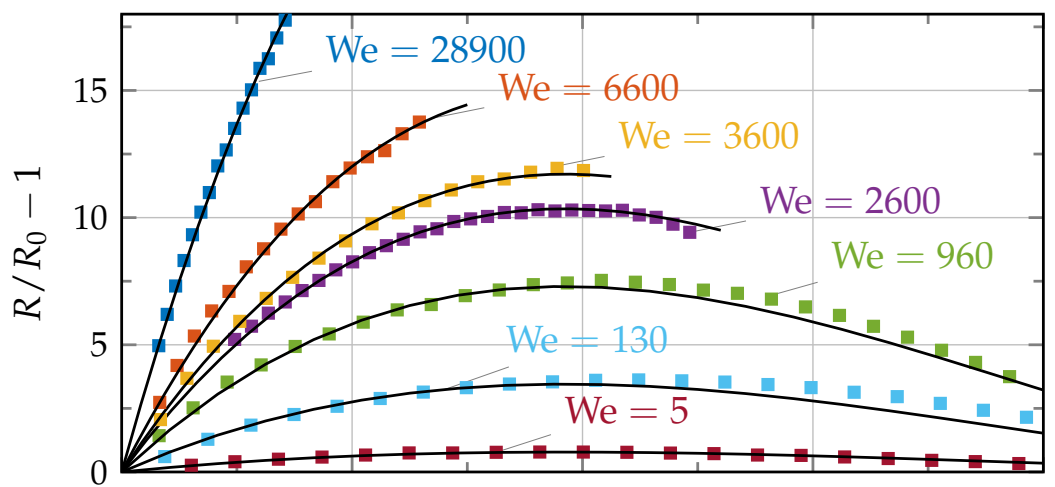

(c)

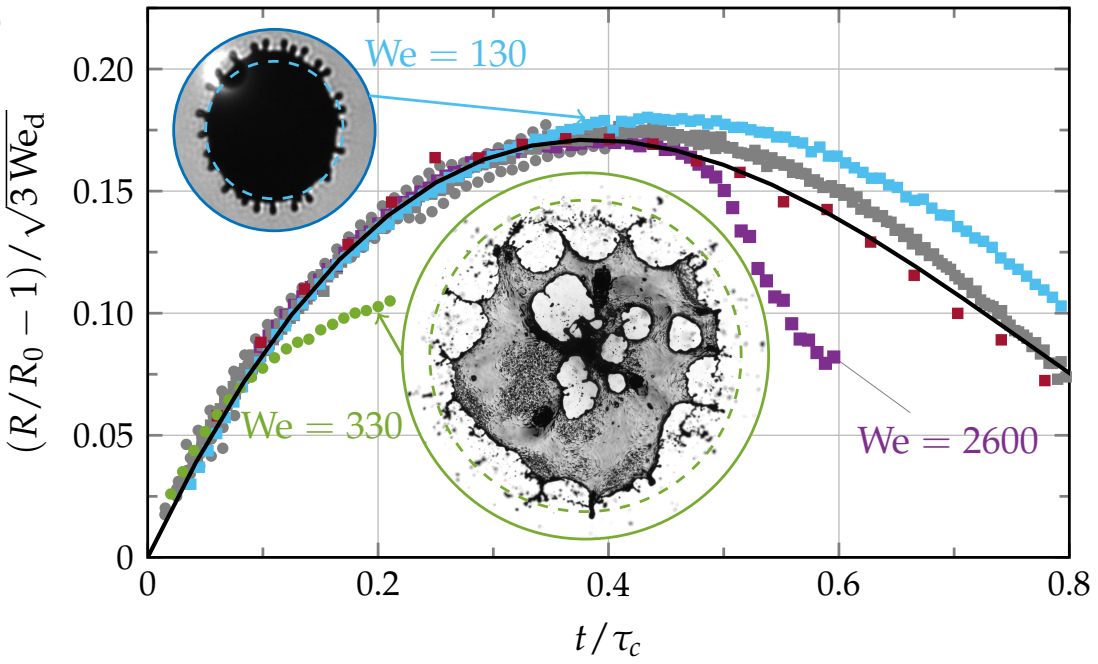

Figure 7.8: Sheet-radius evolution as function of dimensionless time $t / \tau_{c}$ for MEK $((a)$, circle markers in (c)) and tin drops ((b), square markers in (c)). The solid line $(-)$ is the trajectory expected from Eq. (7.8). Experimental curves are shown with a reduced marker density in $(a, b)$ for better visualization. (c) Rescaled experimental data comparing all experiments of $(a, b)$ (gray markers: $\left.\bullet,-{ }^{-}\right)$to the analytical prediction (7.8). Experimental curves are stopped in case the sheet evolution is strongly affected by the fragmentation, which is shown in the two insets for the rim breakup (tin drop at $W e=130$ ) and sheet breakup (MEK drop at We $=330$ ). 
is stable and we only observe the rim breakup, which leads to an apparent over-expansion of the sheet radius in the experiment for $t>t_{\max }$. In the corresponding inset in Fig. 7.8 (c) we see how ligaments are expelled radially outward and do not follow the recoil of the sheet for $t>t_{\max }$. Our image analysis excludes detached ligaments and tiny drops in the calculation of $R$, but ligaments that are connected to the sheet still cause the apparent over-expansion. The same behavior is observed for MEK at We $=90(\bullet)$ in Fig. 7.8 (a). Interestingly, the effect of the rim breakup on the sheet dynamics decreases with increasing Weber number: for We $=960$ (-) the model follows the experimental data points perfectly during the recoil phase (Fig. $7.8(b)$ ), although the rim breakup is observed in the experiment. Indeed, the sheet model (7.8) predicts the rim diameter $b$ and hence the mass contained by the rim to decrease with Weber number as $b / R_{0} \sim \mathrm{We}_{\mathrm{d}}^{-1 / 4}$ [42]. However, with increasing Weber number the sheet fragmentation leads to a deviation between model and experiment, which is illustrated for We $=330\left({ }^{\circ}\right)$ in Fig. 7.8 (c). Holes nucleate in the neck region of the MEK sheet at $t / \tau_{c}<0.2$ and start to grow. Our image analysis is able to deal with holes on the sheet that have not yet reached the outer rim, such that the calculation of $R$ is not affected. However, as holes reach the outer rim and cause the rim to partially detach from the sheet as shown in the inset, the experimentally determined radius $R$ decreases rapidly. The rapid decrease in $R$ due to the neck breakup is also visible for tin sheets, e.g. for We $=2600(-)$ in Fig. 7.8 (c) at $t / \tau_{c}>0.5$. Since the sheet breakup is observed for tin later in time as compared to MEK, this deviation sets in later as well for tin.

The model (7.8) includes a sheet thickness description $h(r, t)$ that applies in a region away from the drop center and reads in terms of this thesis [42]

$$
h(r, t) \sim \frac{R_{0}^{2} \tau_{c}}{\sqrt{\mathrm{We}_{\mathrm{d}}} r} .
$$

Four our discussion on the sheet breakup later in $\S 7.8$ we need a description of the center region where we cannot employ Eq. (7.9). We therefore describe the late-time dynamics of the sheet thickness in a mass-averaged sense as

$$
\begin{aligned}
& \frac{h}{R_{0}} \sim\left(\frac{R}{R_{0}}\right)^{-2}, \text { with } \\
& \frac{R}{R_{0}} \sim \frac{R_{\max }}{R_{0}} \frac{t}{\tau_{c}} \sim \mathrm{We}_{\mathrm{d}}^{1 / 2}\left(\frac{t}{\tau_{c}}\right)^{1 / 2},
\end{aligned}
$$

where we made use in the latter expression of the linear radial expansion, which is valid for $\mathrm{We}_{\mathrm{d}} \gg 1$ and $t \leq t_{\max }$ according to Eq. (7.8). The scaling 
(7.10) is robust as the same relation is found for the neck region, i.e. $r=R(t)$, based on Eqs. (7.9), (7.8), and using $\tau_{c} / \tau_{i} \sim \mathrm{We}_{\mathrm{d}}^{1 / 2}$.

In conclusion, the sheet model $(7.8,7.9)$ captures the kinematics of the deforming drop accurately until the fragmentation severely changes the topology of the deforming liquid body. The kinetic-energy partition $E_{\mathrm{k}, \mathrm{d}} / E_{\mathrm{k}, \mathrm{cm}}$ allows for a treatment of different impact conditions in the MEK and tin system that originate from the different laser-matter interactions. As a consequence, drops in the MEK system expand faster into thin sheets as compared to tin drops for the same Weber number. The sheet model leads to the same scaling for a characteristic sheet thickness in the center and neck region of the sheet, where we observe the first hole nucleation during the sheet breakup. With a solid description of the kinematics for the liquid sheet at hand we now turn to the rim breakup in $\S 7.7$ and the sheet breakup in $\S 7.8$.

\subsection{Rim breakup}

Our goal is to find a prediction for the number of ligaments $N_{\ell}$ that are expelled radially outward as a result of the rim breakup. To this end, we determine at what time $t_{\ell}$ a Rayleigh-Plateau and Rayleigh-Taylor instability manifest themselves on the rim. From the sheet radius and the characteristic wavenumber $k_{\ell}$ at that time follows then the number of ligaments $N_{\ell} \sim$ $R\left(t_{\ell}\right) k_{\ell}$. Based on the similarity between our expanding sheet and the one occurring after the mechanical impact of a drop on a solid, we follow a similar approach as Villermaux and Bossa [42].

The evolution of the rim breakup that finally results in the radial expulsion of ligaments is illustrated for tin at $\mathrm{We}_{\mathrm{d}}=140$ in Fig. 7.9. Corrugations with an amplitude $\xi$ develop on the rim and are visible in the experiments initially as mere noise and later as a perturbation with a characteristic wavenumber $k_{\ell}$. We define the latter moment as the time $t_{\ell}$ of rim breakup. Once the ligaments have manifested themselves on the rim, their number $N_{\ell}$ only changes due to the compression of the rim at $t>t_{\max }$ when the sheet starts to recoil: individual ligaments that are still attached to the sheet approach each other such that they merge at their base as shown in Fig. 7.9 (c, d). The corresponding decrease of $N_{\ell}$ over time in Fig. 7.9 (a) is thus not a result of an instability rearranging the fluid, but a mere consequence of the compression of the rim.

We observe the development of ligaments before the sheet starts to recoil at $t_{\max }$ as shown in Fig. 7.10: the moment we observe the manifestation of the corrugations as radial ligaments decreases with increasing Weber number. Following Villermaux and Bossa [42], we describe the rim as a liquid cylinder of diameter $b \sim R_{0} \mathrm{We}_{\mathrm{d}}^{-1 / 4}$ and neglect its curvature, which is valid for our 

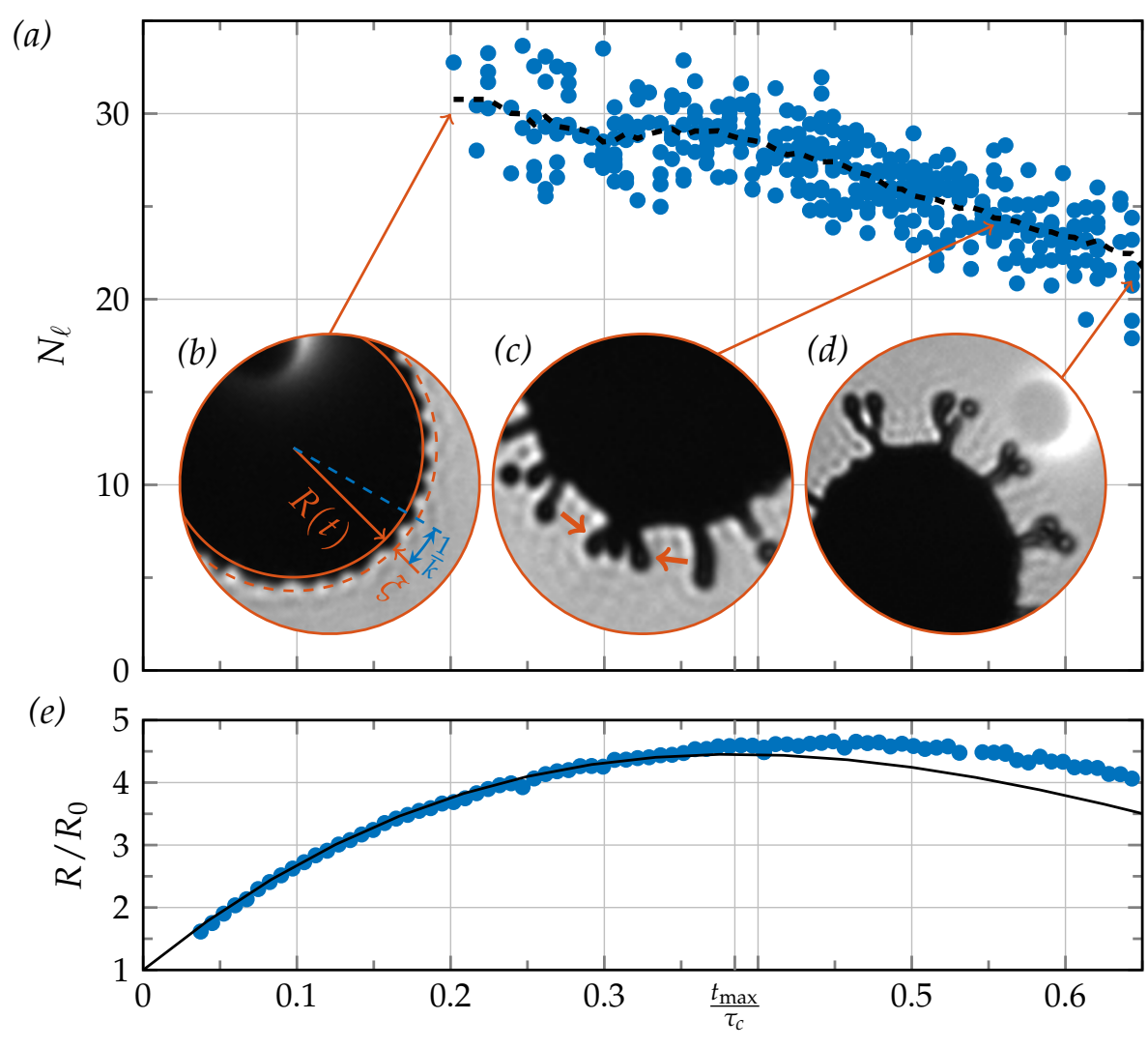

$t / \tau_{c}$

Figure 7.9: Evolution of the rim breakup for $\mathrm{We}_{\mathrm{d}}=140$ as function of dimensionless time $t / \tau_{c}$ obtained for a tin experiment that exhibits a near-perfect axisymmetric expansion. (a) Total number of ligaments $N_{\ell}$, where markers (•) indicate individual realizations of the experiment and the dashed line $(---)$ is a running average. The inset (b) shows the sheet radius $R(t)$, the amplitude $\xi$, and the wavenumber $k$ of the corrugation as observed at $t / \tau_{c}=0.2$. During the recoil of the sheet at $t>t_{\max }$ two or more ligaments may merge as shown in inset (c) and (d). (e) Sheet radius $R(t)$ for each realization $(\bullet)$ and the trajectory $(-)$ from Eq. (7.8).

experiments since $k_{\ell} R \gg 1$. The rim is stretched over time at a rate $\dot{R} / R$ and sits in a decelerating frame with a time-dependent acceleration $-\ddot{R}(t)$. Two destabilization mechanisms play a role in such a case. First, the capillary destabilization by a Rayleigh-Plateau mechanism affects the rim of constant diameter $b$. The associated timescale of the capillary instability is then $\left(\rho b^{3} / \gamma\right)^{1 / 2} \sim \tau_{c} \mathrm{We}_{\mathrm{d}}^{-3 / 8}$, which is in agreement with our experimental observation in Fig. 7.10. Second, a Rayleigh-Taylor mechanism is at play, 


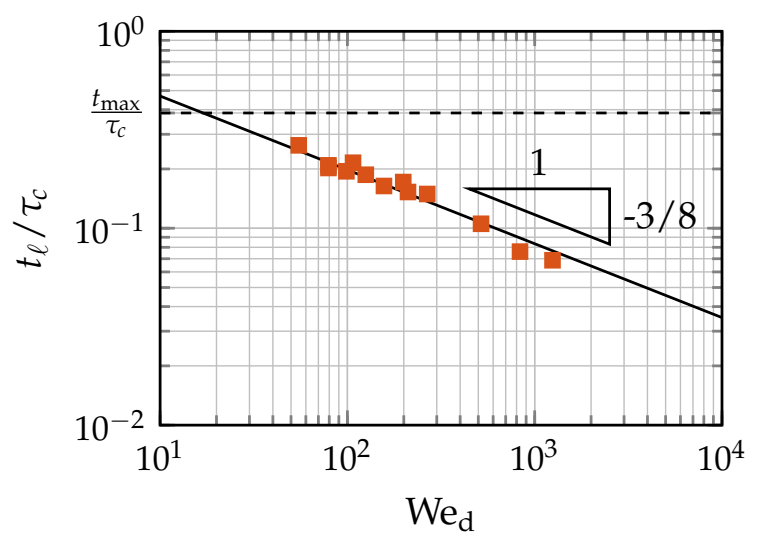

Figure 7.10: The time $t_{\ell}$ when corrugations similar to the ones in Fig. 7.9 (b) become visible on the bordering rim as a function of Weber number $\mathrm{We}_{\mathrm{d}}$. The data is acquired manually from a subset of tin experiments that are recorded at identical camera and lighting conditions to exclude an influence of the image resolution. The prefactor of the scaling law $(-)$ is 1.1 .

where an associated body force proportional to $-\rho \ddot{R}$ pushes the fluid particles in the rim radially outward. The growth rate of the corrugation in a local frame tangent to the rim is then [42]

$$
\frac{\mathrm{d}}{\mathrm{d} t} \ln \left(\frac{\xi}{R}\right)=-\frac{\dot{R}}{R}+\operatorname{Re}(-i \omega),
$$

where $\omega$ is the instantaneous growth rate. The first term on the right-hand side in Eq. (7.12) is the stretching of the rim that actually damps the growth of the perturbation during the expansion of the sheet for $t<t_{\max }$. The timescale of the damping is set by $\tau_{c}$, independent of the Weber number [42]. The second term on the right-hand side in Eq. (7.12) amplifies the corrugation with an instantaneous growth rate $\omega$ that depends on $\mathrm{We}_{\mathrm{d}}$. The associated timescale of the amplification is $\omega^{-1} \sim\left(\rho \ddot{R}^{3} / \gamma\right)^{-1 / 4} \sim \tau_{c} \mathrm{We}_{\mathrm{d}}^{-3 / 8}$, which is the same timescale as found for the Rayleigh-Plateau mechanism. In our experiments, $W_{\mathrm{d}} \gg 1$ such that the timescale of the Rayleigh-Taylor instability is separated from the expansion dynamics. As a consequence, the damping due to the stretching is irrelevant for large Weber numbers allowing for the instability to manifest itself on the rim at early times, that is

$$
t_{\ell} \sim \tau_{c} \mathrm{We}_{\mathrm{d}}^{-3 / 8} .
$$


The wavenumber $k_{\ell}$ for which $\omega$ in Eq. (7.12) reaches a maximum is $k_{\ell} \sim$ $(-\ddot{R} \rho / \gamma)^{1 / 2}=\mathrm{We}_{\mathrm{d}}^{1 / 4} / R_{0}$ [42]. From the timescale $t_{\ell}$ of the destabilization and the corresponding wavenumber $k_{\ell}$ we can determine $N_{\ell}$. The expansion dynamics (7.8) can be approximated for $t \leq t_{\max }$ by $R / R_{0} \sim \mathrm{We}_{\mathrm{d}}^{1 / 2} t / \tau_{c}$ leading finally to

$$
N_{\ell} \sim k_{\ell} R\left(t_{\ell}\right) \sim \mathrm{We}_{\mathrm{d}}^{3 / 8}
$$

The scaling exponent of $3 / 8$ in Eq. (7.14) deviates from the scaling of $3 / 4$ in terms of the Weber number as proposed by Villermaux and Bossa [42]. In their study, the time of maximum expansion is assumed to be the characteristic time of the destabilization, i.e. $t_{\ell}=t_{\max } \sim \tau_{c}$. And indeed, in $\S 7.6$ we saw that at $t \sim t_{\max }$ the rim breakup affects the sheet kinematics considerably, but we observe the manifestation of the instability at an earlier point in time leading to the different scaling with Weber number.

We validate the scaling (7.14) experimentally as shown in Fig. 7.11. Since the number of ligaments in the experiments only changes by the rim compression for $t>t_{\max }$, we can obtain $N_{\ell}$ as function of $W \mathrm{e}_{\mathrm{d}}$ by simply counting the number of ligaments that are pointing radially outward during $t_{\ell} \leq t \leq t_{\max }$ which is illustrated in Fig. $7.11(\mathrm{a}-\mathrm{d})$. The obtained scaling with $\mathrm{We}_{\mathrm{d}}$ in Fig. 7.11 (e) is in good agreement with our prediction (7.14) with a prefactor of 4.4. The limited range in $\mathrm{We}_{\mathrm{d}}$ for which data can be obtained for the MEK experiments prevents a detailed comparison for the two systems in terms of the scaling exponent. The sheet breakup takes place in the MEK system much earlier in comparison to the tin system as we already mentioned in §7.6. The neck breakup in direct vicinity to the rim limits then the range in $\mathrm{We}_{\mathrm{d}}$ for which can obtain a reliable measurement of $N_{\ell}$ in the MEK system. However, the MEK data points ( $\bullet$ ) that we did mange to obtain follow the same scaling (7.14) with the same prefactor as the tin data $(\square)$. Hence, we conclude that the difference in rim breakup between MEK and tin is completely captured by the rescaled Weber number $\mathrm{We}_{\mathrm{d}}$ that accounts for the different driving mechanism, in particular the effect of the plasma dynamics on the expansion of the tin sheets. 
(a)

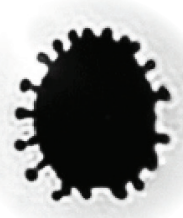

$\mathrm{We}_{\mathrm{d}}=230$ (b)

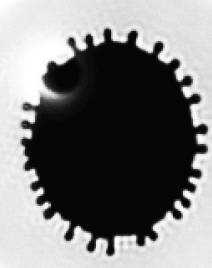

$\mathrm{We}_{\mathrm{d}}=410$ (c)

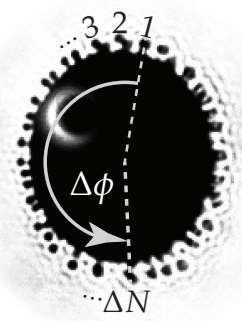

$\mathrm{We}_{\mathrm{d}}=650$ (d)

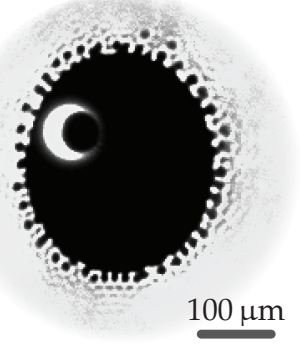

$\mathrm{We}_{\mathrm{d}}=1030$

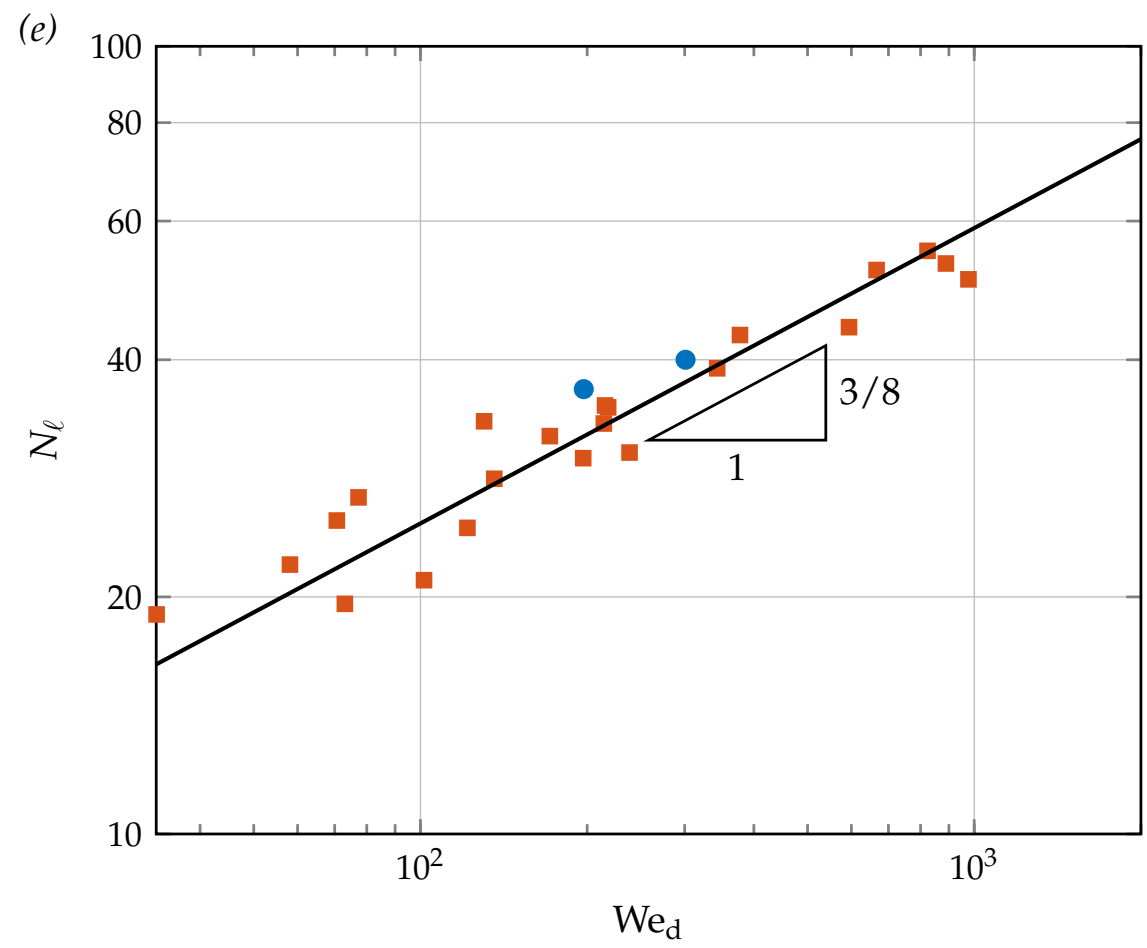

Figure 7.11: (a-d) Radial expulsion of ligaments during the rim breakup for increasing Weber number $\mathrm{We}_{\mathrm{d}}$ (left to right). The back-view images are taken from experiments with tin drops that exhibit a near-perfect axisymmetric expansion. The optical resolution may limit the detection of ligaments to a fraction $\Delta \phi / 2 \pi$ of the rim as illustrated in (c). In such cases, the expected total number of ligaments $N_{\ell}$ is estimated by $N_{\ell}=2 \pi(\Delta N-1) / \Delta \phi$. (e) Number of radially expelled ligaments $N_{\ell}$ as function of the Weber number $W_{d}$ for tin ( $\square$ ) and MEK drops (๑). The data for MEK is limited to two experiments, since the early hole nucleation in the neck region affects the rim breakup and prevents an accurate measurement for larger Weber numbers. The prefactor of the scaling law (-) is 4.4 . 


\subsection{Sheet breakup}

The questions we aim to answer in the analysis of the sheet breakup are analogous to the rim breakup: When does the sheet break and which characteristic wavenumber is selected during the breakup? We already found an agreement in $\S 7.5$ between our observations for the nucleation, growth, and merging of holes and an analogous process for a manually prepared liquid film that is accelerated by a shockwave [117]. The analogy suggest the same mechanism as cause of the sheet destabilization for the laser impact on a drop: an impulsive Rayleigh-Taylor instability. However, the sheets in our experiments are not subject to a direct acceleration of either one of their interfaces. Only the spherical drop is accelerated on the timescale $\tau_{e}$ and the sheets, as well as a potential Rayleigh-Taylor instability, must develop simultaneously until the sheet breaks on the timescale $\tau_{c}$. As a consequence, we study the sheet breakup in $\S 7.8 .1$ in more detail to convince ourselves that it shows typical features of an instability before we propose a model in $\S 7.8 .2$ to answer our questions.

\subsubsection{Observations}

We first recognize that the Weber number not only sets the expansion dynamics (7.8) and (7.9) when rescaled by the energy partition $E_{\mathrm{k}, \mathrm{d}} / E_{\mathrm{k}, \mathrm{cm}}$, but also represents the initial drop acceleration $a$ on the timescale $\tau_{e}$. In comparison to $\tau_{i}$ and $\tau_{c}$ the acceleration can be considered impulsive and is then given by $a \sim U / \tau_{e}=R_{0} /\left(\tau_{c} \tau_{e}\right) W \mathrm{We}^{1 / 2}$. Since $R_{0}$ and $\tau_{c}$ are kept constant in each experimental setup and $\tau_{e}$ is found to be constant as well for both vapor-driven and plasma-driven drops, $\mathrm{We}^{1 / 2}$ is a direct scale for the impulsive acceleration in our experiments. As a result, our observations cannot distinguish between a stronger accelerations on the one hand and a faster expansion on the other hand. Both are coupled and cannot be set independently, unless $E_{\mathrm{k}, \mathrm{d}} / E_{\mathrm{k}, \mathrm{cm}}$ could be controlled experimentally in both systems.

Figure 7.11 shows the breakup of MEK sheets for one decade in Weber number. In case the laser impact is severe enough a minimum Weber number is achieved to observe the sheet breakup. A further increase in Weber number (Fig. $7.11(\mathrm{~b}-\mathrm{e})$ ) leads to a more severe fragmentation process as the number of holes $N_{b}$ that pierce the sheet per unit area $A$ and the corresponding wavenumber $k_{b} \sim\left(N_{b} / A\right)^{1 / 2}$ are increased. Our observation for the increase in $k_{b}$ with We is a clear signature of a fluid-dynamics instability and in agreement with the study on liquid films by Bremond and Villermaux [117]. The mode selection, i.e. the characteristic wavenumber $k_{b}$ observed in their experiments, was captured in the analysis by an impulsive Rayleigh-Taylor instability. In 
addition, the characteristic time of the instability development was found to be a function of We as well. Our experiments also reveal a faster fragmentation process for an increase in Weber number, see Fig. 7.11 (b-e). However, only the analysis can reveal whether the time dependency in our experiments is a result of a stronger acceleration or mere consequence of faster sheet dynamics.

A characteristic scale for the sheet thickness $h_{\mathrm{b}}$ at the moment of breakup shall help to exclude other mechanisms than a fluid-dynamic instability as cause of the destabilization. The minimum Weber number for sheet breakup to occur in the MEK system (Fig. 7.11 (a)) translates to a radial sheet expansion of $R / R_{0}>6$ (similar expansions are required to cause destabilization for tin sheets). The scaling (7.10) leads then to the typical sheet thickness $h_{\mathrm{b}} / R_{0} \sim$ $10^{-2}$. We confirm by high-speed recordings of individual piercing events on MEK sheets that the hole-opening speed agrees with the Taylor-Culick speed $v \sim \sqrt{\gamma / h_{\mathrm{b}} \rho} \sim 1 \mathrm{~m} / \mathrm{s}$ [127] as set by our estimate of $h_{\mathrm{b}}$. The typical scale of $h_{\mathrm{b}}$ in absolute terms is then a few tens of micrometer for the MEK sheets and a fraction of a micrometer for the tin sheets. We prevent solid impurities of such a length scale to enter the initial MEK drops by an appropriate filtration as explained in $\S 7.3$. Furthermore, we exclude by high-speed recordings for selected experiments that the breakup is caused by individual fragments impacting on the sheet. The ejected mass during the early jetting phenomena, a likely candidate for fragments, travels at a velocity larger than the center-ofmass velocity $U$ of the expanding sheet preventing any collision with the sheet at later times. Van der Waals forces can be important when the film thickness is in the range of several tens of nanometer [128], but even the tin sheets at a micrometer-scale are not yet thin enough for this effect to be relevant. The combination of two liquid systems at vastly different scales is to our advantage here: any mechanism proposed as cause for the breakup must hold for both systems, the framework of an impulsive Rayleigh-Taylor instability being the most likely one in view of our observations.

A Rayleigh-Taylor instability amplifies initial perturbation until their amplitude severely affects the topology of the liquid body. Following Bremond and Villermaux [117], the sheets in our experiment are then most likely pierced when the amplitude of the instability is of the order of the local sheet thickness. A fragmentation process based on the amplification of initial perturbations, which can be as small as the thermal noise in a system [50], is expected to show a stochastic nature. We therefore elucidate whether the hole nucleation and the formation of the characteristic web of ligaments (Fig. 7.11(b-e)) is set by deterministic or stochastic influences, or a combination of the two.

The patches of corrugations that precede the hole formation as described in $\S 7.4$ are also visible in Fig. $7.11(\mathrm{~b}-\mathrm{e})$ and are an indication for the destabilization of the liquid sheet to occur. Vice versa, corrugations with a high 
spatial frequency are absent on stable sheets (Fig. 7.11(a)). We observe no direct relation between the spatial frequency of the corrugation $k_{\text {corr }}$ and the wavenumber $k_{b}$ of holes piercing the sheet in a corrugated area: only a few holes pierce a corrugated area on MEK sheets, i.e. $k_{\text {corr }} \gg k_{b}$. However, we can use the appearance of corrugations as an indication where holes are likely to occur. To this end, we use an image-analysis algorithm that is sensitive to spatial frequencies much larger than the hole density, see Fig. 7.12 (a). From the data of approximately 100 experimental realizations, one of which is shown in Fig. 7.12 (b), we obtain the probability density function (PDF) for likely areas of hole nucleation in radial direction as shown in Fig. 7.12 (c). Not surprisingly, our quantitative analysis recovers the areas of hole nucleation that we already identified visually in $\S 7.4$ : the neck and center region of the sheet breakup. With the two regions identified by a bimodal gaussian distribution we recover the PDF in azimuthal direction for each radial region (Fig. 7.12(d)). Also here, we recognize a deterministic influence with three preferred locations of hole piercing in the center region and approximately six preferred areas of destabilization in the neck region. In agreement with these findings, the final web of ligaments preserves the deterministic influence as illustrated for a large Weber number We $=2000$ in Fig. 7.13.

The deterministic facets of the sheet fragmentation are not in contradiction to a Rayleigh-Taylor mechanism. The instability can very well be the cause for the hole piercing but its visual appearance is convoluted by a deterministic profile in the sheet thickness $h$. We clearly observed a radial dependency in the sheet thickness that needed to be included in our quantitative description of the expansion dynamics in $\S 7.6$ to accurately describe our experimental data. In addition, we noticed azimuthal modulations in the sheet thickness

Figure 7.11: (Figure is found on the following page.) Sheet breakup for increasing Weber number We in back-view images taken from MEK experiments. (a) The sheet is smooth and starts to recoil from its maximum radius $R_{\max } / R_{0}=6$ reached at $t / \tau_{c}=2 / \sqrt{27} \approx 0.38$, the moment the image is taken. The rim breakup leads to the formation of ligaments but the breakup of the thin sheet itself is not observed. A slight increase in the Weber number leads however to single breakup events on the sheet (not shown). (b-e) The sheets are first pierced near their neck and in the center before $R_{\max }$ is reached. The images are taken shortly after the first piercing event to allow for a characteristic hole density to develop, since the piercing is subject to fluctuations. The dimensionless time of each image is decreasing $t / \tau_{c}=0.3,0.2,0.15,0.12(\mathrm{~b}-\mathrm{e})$ with increasing Weber number. The shadowgraph visualization with a small numerical aperture is sensitive to any light refraction such that corrugations on the thin sheet just before breakup become visible. With increasing We, we observe a larger hole density resulting in a finer web of ligaments. 


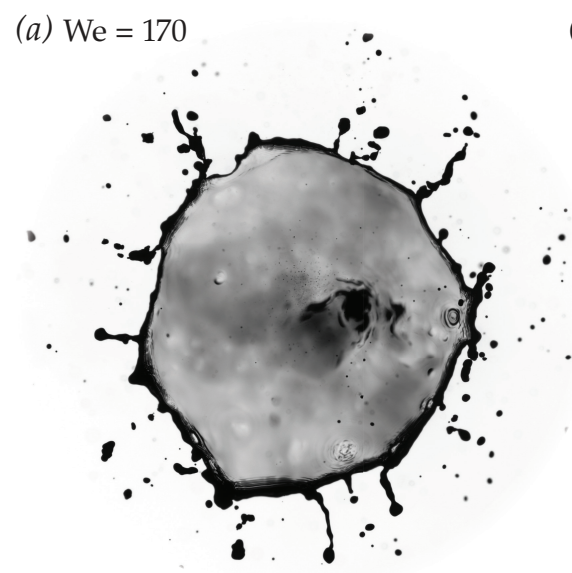

(c) $\mathrm{We}=530$

(b) $\mathrm{We}=330$

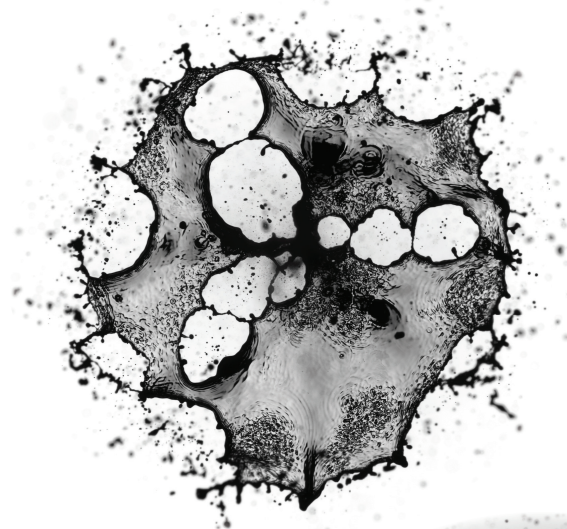

(d) $\mathrm{We}=1180$.
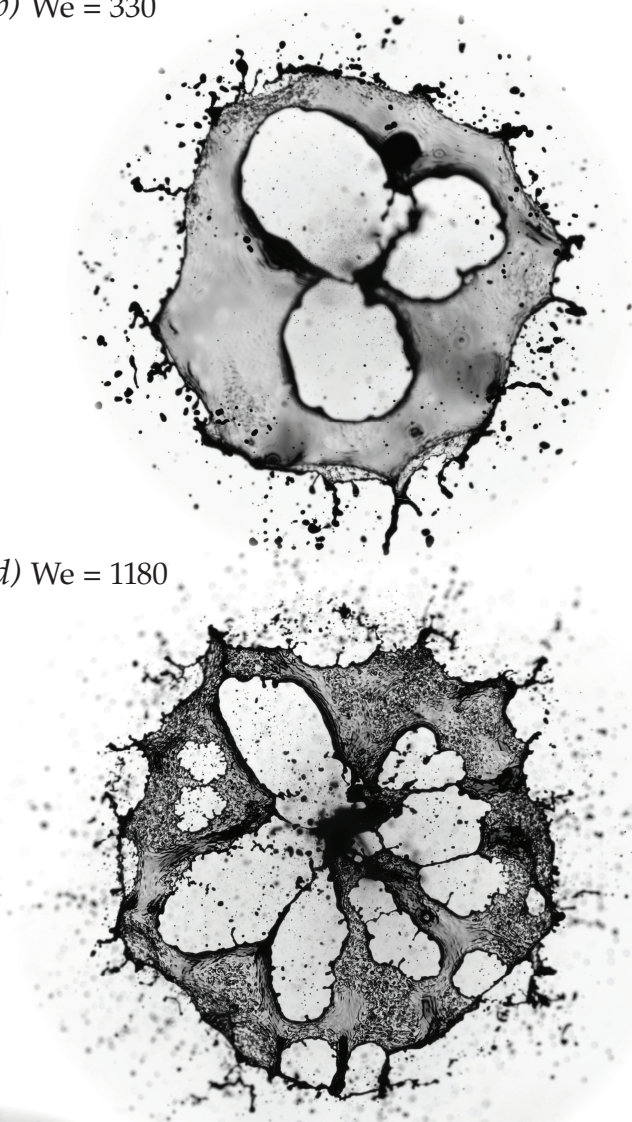

(e) $\mathrm{We}=2000$

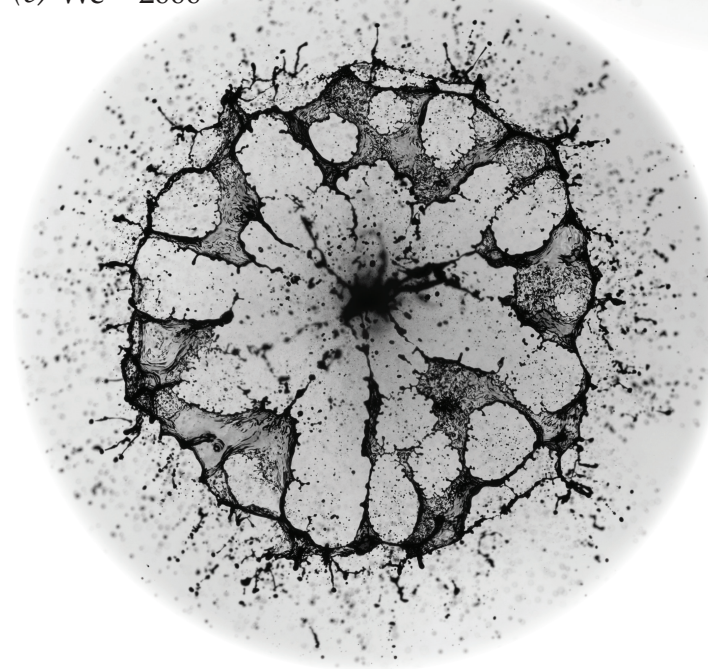

Figure 7.11: (Continued from the previous page.) 
(a)

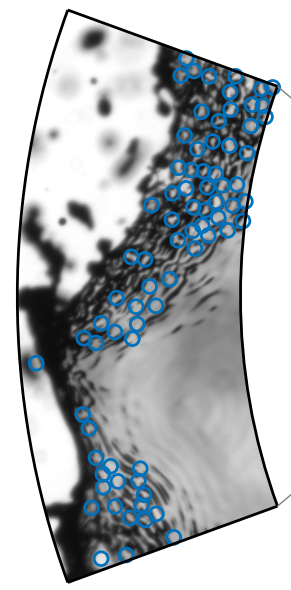

(c)

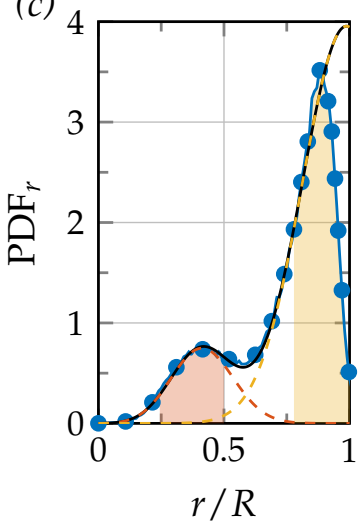

(b)

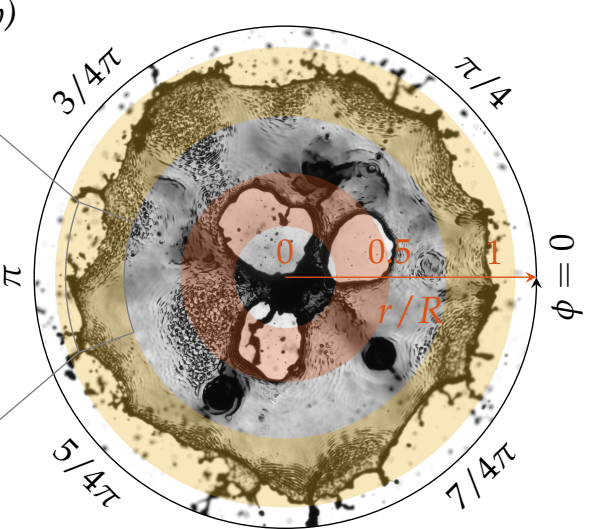

(d)

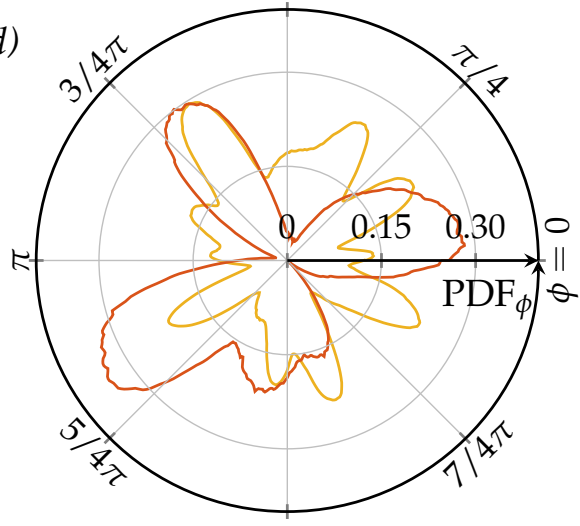

Figure 7.12: Hole nucleation on MEK sheets at We $=440$. (a) Magnified section of the sheet in (b) illustrating the result of an algorithm to detect corrugations that precede the sheet breakup: a cross-correlation of the image and a gaussian kernel with a standard deviation $\sigma \sim 1 / k_{\text {corr }}$ identifies local corrugations ( $(\circ)$, where $1 / k_{\text {corr }}$ is a scale for the spatial frequency of the corrugations. (b) Sheet from a single realization of the experiment, where the center and neck region of the sheet breakup as identified by the analysis shown in (c) are highlighted (neck: $\square$, center: $\square$ ). (c) Probability density function (PDF) for the radial location $r / R$ of the local sheet corrugations found in approximately 100 realizations of the experiment. The PDF is approximated by PDF $=2 r / R g(r)$, where $g(r)$ is the radial modulation that describes the deviation of the hole nucleation location from a random distribution. The experimental data $(-)$ is well described by a two-component Gaussian mixture model $g\left(r \mid \mu_{i}, \sigma_{i}\right)(-)$ with $\mu_{i}$ and $\sigma_{i}$ being the mean and standard deviation of the radial location of hole nucleation. The highlighted areas, i.e. $\mu_{i}-\sigma_{i} \leq r / R \leq \mu_{i}+\sigma_{i}$, illustrate the preferred hole locations in the center $(\square, \mu=0.37, \sigma=0.13)$ and the neck region $(\square$, $\mu=0.96, \sigma=0.180$ ) of the sheet. (d) PDF of the azimuthal position $\phi$ of preferred hole locations for the center (- ${ }_{-}$) and neck region $(-)$of the sheet breakup. 
(a)

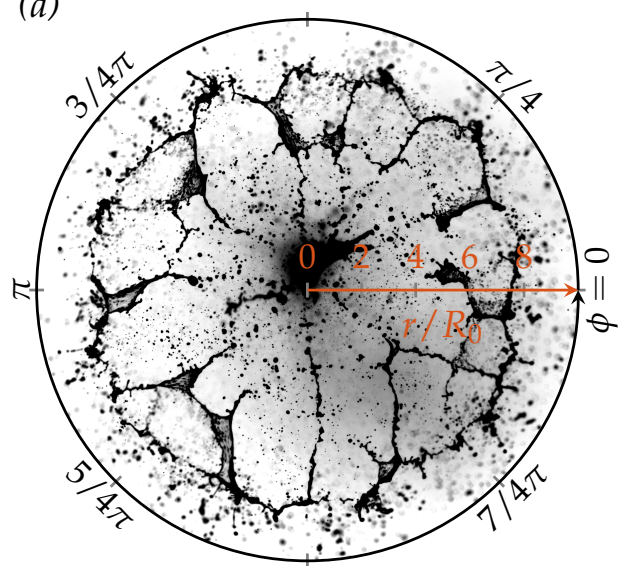

(b)

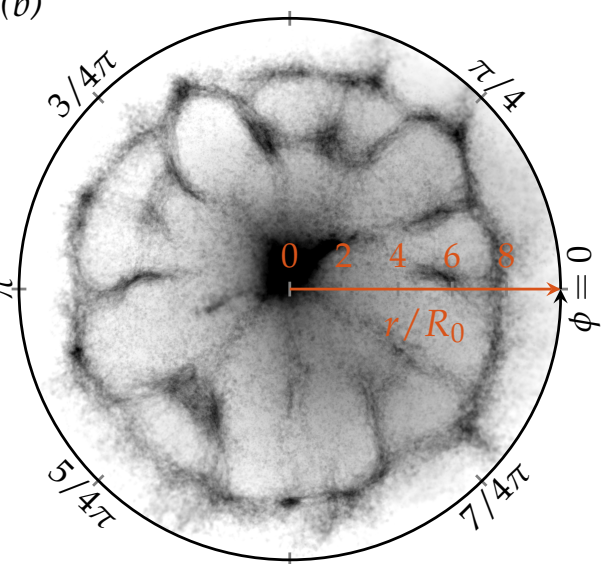

Figure 7.13: (a) Back-view of a single experiment for an MEK drop at We $=2000$ and $t / \tau_{c}=0.15$. The nucleation, growth, and merging of holes on the sheet leads to a web of ligaments. (b) Overlay of 31 back-view images at $t / \tau_{c}=0.15 \pm 0.006$ for the same experimental condition as in (a) but taken from separate, independent realization of the same experiment. The gray value in the overlay image is a measure for the probability that ligament are present at the same position in all 31 realization of the same experiment, where a black pixel means that in all experiments a ligament is found at a particular position. The overlay reveals a deterministic influence on the final web of ligaments during the sheet fragmentation.

in $\S 7.4$. Although these modulations do not affect the expansion dynamics considerably, the sheet thickness varies locally during the late-time expansion. The physical cause for azimuthal modulations can be found in the driving mechanism as explained in §7.2: the vapor-driven MEK drops are subject to azimuthal modulations in the initial acceleration of about $\pm 10 \%$ in amplitude. As these modulations are deterministic, that is fixed in the lab reference frame, the fragmentation also shows deterministic aspects.

To observe the stochastic nature of hole nucleation we then need to find an experiment where the characteristic wavelength of hole nucleation is small in comparison to the spatial extent $L$ of local thickness variations, that is $k_{b} L \gg 1$. In Fig. 7.14 we show a corresponding case for the center breakup of a tin sheet at $\mathrm{We}=30000$. The hole nucleation with a wavenumber $k_{b}$ in the center of the sheet is observed to take place on an area with radial extent $L_{c}$ such that $k_{b} L_{c} \gg 1$. Such a case allows us to sample the process of hole nucleation in large numbers to obtain unbiased statistics, i.e. unaffected by large-scale variations in the sheet thickness. Our analysis for the location of piercing events recovers a distribution as expected for a stochastic process (Fig. $7.14(\mathrm{~b}, \mathrm{c})$ ). With the experimental observation of random hole nucleation 

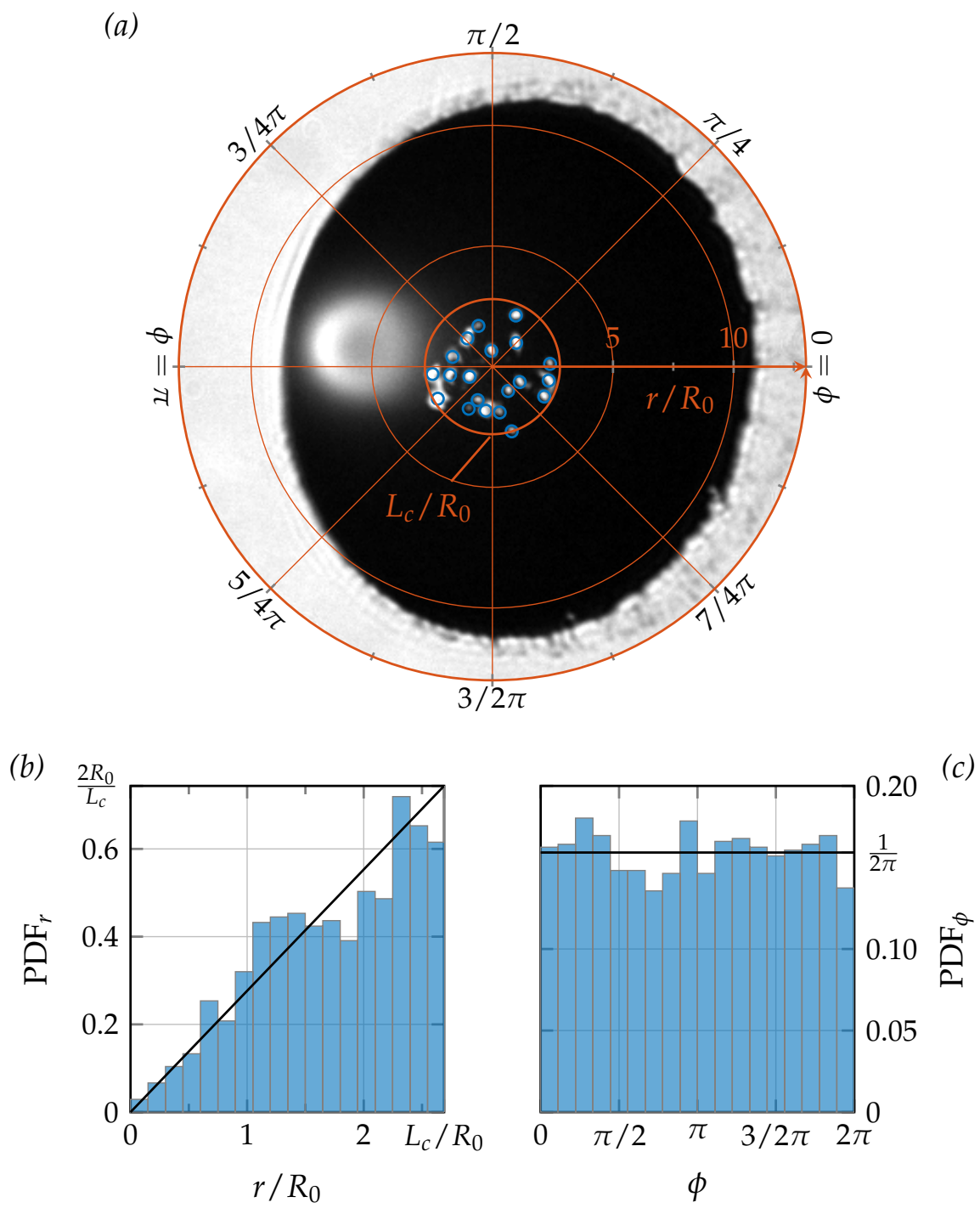

Figure 7.14: Hole nucleation in the center of tin sheets at $\mathrm{We}=30000$. (a) A single realization of the experiment with individual holes highlighted (०) as detected by an image-analysis algorithm that is sensitive to variations in the gray value. Approximately 100 realizations of the experiment lead to a spatial distribution of hole nucleation events, where the area that holds $90 \%$ of these events is marked by the circle $L_{c} / R_{0}=2.7$ in (a). For this area, the radial (b) and azimuthal distribution (c) of nucleation events is obtained. The experimental distributions ( $\square$ ) agree with the corresponding prediction for a random nucleation process $(-)$, where it follows from geometric arguments: $\mathrm{PDF}_{r}=2 r / L_{c}$ and $\mathrm{PDF}_{\phi}=1 /(2 \pi)$. The wavenumber $k_{b}$ of hole nucleation is found from the total number of holes $N_{b}$ identified in $n$ realizations of the experiments to be $k_{b} R_{0}=\left(R_{0}^{2} N_{b} /\left(\pi n L_{c}^{2}\right)\right)^{1 / 2}=0.86$. 
we conclude on the destabilization mechanism. The physical mechanism of the sheet breakup is a fluid-dynamic instability that is affected by large-scale variations in the sheet thickness. In particular, we anticipate a Rayleigh-Taylor instability that is driven by the impulsive acceleration of the drop at $\tau_{e}$. Our analysis in the next section then leads to a prediction for the characteristic time of destabilization, which we can validate experimentally.

\subsubsection{Model derivation and comparison to experiment}

Here, we describe the model to predict the sheet breakup taking into account the characteristics of the laser impact. We identified a Rayleigh-Taylor instability as mechanism for the sheet destabilization that is initiated by the impulsive acceleration of the drop surface at $\tau_{e}$. Any perturbations that are present on the accelerated interface of the drop are then amplified during this early-time of sheet breakup. At later times $t>\tau_{e}$ the perturbations continue to evolve in absence of an external acceleration, while still affected by capillary restoring forces as the overall topology of the drop is changing as well. We aim to capture two characteristics of the drop evolution in our analysis: the change from a spherical drop to a sheet on the timescale $\tau_{i}$ and the expansion of the drop into a thin sheet at $\tau_{c}$, both are a late-time consequence of the initial acceleration. Our arguments are based on the analysis by Bremond and Villermaux [117] for thin liquid films that were also accelerated impulsively. In our case, the sheets are then most likely pierced when the perturbation amplitude is of the order of the sheet thickness. We therefore trace the perturbation amplitude from the early-time $\tau_{e}$ to the late-time $\tau_{c}$, where we observed the breakup in the experiments.

Figure 7.15 sketches the evolution of a Rayleigh-Taylor instability on a deforming drop in three distinct phases. The first phase (Fig. 7.15 (a)) describes the acceleration of the drop, where in comparison to $\tau_{i}$ and $\tau_{c}$ the acceleration can be considered impulsive and is then given by a step function equal to $a \sim U / \tau_{e}=R_{0} /\left(\tau_{c} \tau_{e}\right) W e^{1 / 2}$ for $0 \leq t \leq \tau_{e}$ and equal to 0 for $t>\tau_{e}$. In our model the drop is assumed to be a sheet with thickness $h_{0} \sim R_{0}$ and density $\rho$ that is surrounded by a gas phase of negligible density. One interface of the sheet is then accelerated perpendicular to its surface. The acceleration $a$ amplifies any initial modulation of the surface, which can be represented by the Fourier modes

$$
\eta(s, t)=\eta_{0} f(t) \mathrm{e}^{i k s},
$$

where $\eta_{0}$ is the initial amplitude and $s$ a generalized coordinate tangent to the sheet in the following analysis. The temporal evolution $f(t)$ is determined by the instantaneous growth rate $\omega(k)$, i.e. the dispersion relation specific 
(a)

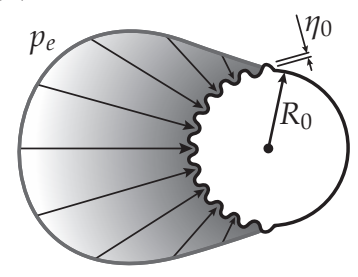

(b)

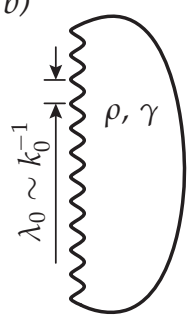

$\tau_{e}<t \leq \tau_{i}$ (c)

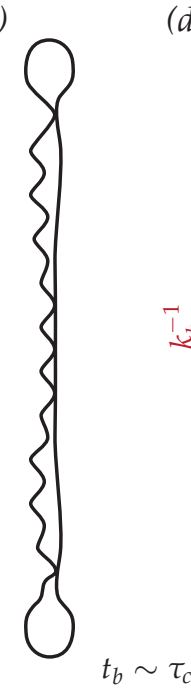

(d)

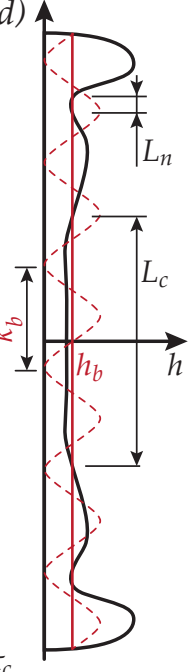

Figure 7.15: Sketch of a model to describe the evolution of an impulsive RayleighTaylor instability on a deforming drop. (a) One side of the drop is accelerated perpendicular to the surface by the pressure $p_{e}$ on the timescale $\tau_{e}$. The acceleration $a$ amplifies the Fourier modes of initial amplitude $\eta_{0}$ and wavenumber $k_{0}$. (b) The drop deforms into a sheet on the timescale $\tau_{i}$ in absence of any external acceleration. (c) The sheet expands considerably until it breaks at the time $t_{b}$ when the perturbation amplitude is of the order of the sheet thickness $h_{b}$. (d) A thickness profile showing two regions of size $L_{n}$ and $L_{c}$ where the criterion for breakup is fulfilled and holes are expected to nucleate with a characteristic wavenumber $k_{b}$.

to each of the three phases. In the second phase (Fig. 7.15(b)), the drop is not accelerated any more but the Fourier modes continue to exist on a drop that evolves into a sheet on the timescale $\tau_{i}$. The third phase (Fig. 7.15 (c)) is characterize by the large expansion of the sheet on the timescale $\tau_{c}$, during which we observe the actual breakup. The sheet is then pierced when the thickness modulations are of the order of the sheet thickness [117], compare also Fig. $7.15(\mathrm{~d})$. We neglect in our analysis the local thickness variations (marked by $L_{c}$ and $L_{n}$ in Fig. $7.15(\mathrm{~d})$ ) that lead to the preferred areas of hole nucleation as seen in the previous section, since the underlying mechanism of destabilization is unaffected by those imperfections. Consistently, we make use of the robust scaling (7.10) for the overall kinematics of the sheet.

Lengths and times are made non-dimensional in the analysis by the initial drop radius $R_{0}$ and capillary time $\tau_{c}$,

$$
\hat{\omega}=\omega \tau_{c}, \hat{t}=\frac{t}{\tau_{c}}, \hat{k}=k R_{0}, \text { and } \hat{k}_{c}=\sqrt{\frac{\rho a R_{0}^{2}}{\gamma}}
$$


where $\hat{k}_{c}$ is the capillary wavenumber. Following Bremond and Villermaux [117], we treat the acceleration $a=R_{0} /\left(\tau_{c} \tau_{e}\right) \mathrm{We}^{1 / 2}$ in the first phase as impulsive, i.e. we assume $1 / \hat{\omega}_{1} \gg \hat{\tau}_{e}$. Since $\hat{k}_{c, 1}=\mathrm{We}^{1 / 4} \hat{\tau}_{e}{ }^{-1 / 2} \gg 1$, the sheet can be considered thick with respect to the capillary length, which leads to the dispersion relation [117]

$$
\hat{\omega}_{1}^{2}=\hat{k}^{3}-\hat{k}_{c, 1}^{2} \hat{k}
$$

The initial conditions for the shape function $f(t)$ in Eq. (7.15) are

$$
f_{1}(\hat{t}=0)=1 \text { and } f_{1}^{\prime}=\frac{\mathrm{d} f_{1}}{\mathrm{~d} \hat{t}}=0,
$$

such that we allow all unstable modes that fit inside the drop, that is $1 \leq$ $\hat{k} \leq \hat{k}_{c, 1}$, to grow with the same initial amplitude and zero initial speed. The solution of $f_{1}(t)$ then follows from $[117,129]$

$$
\frac{\mathrm{d}^{2} f_{1}}{\mathrm{~d} \hat{t}^{2}}=-\hat{\omega}_{1}^{2} f_{1}(\hat{t})
$$

and is found to be

$$
f_{1}(\hat{t})=\cos \left(\hat{k}^{3 / 2} \hat{t}\right)+\frac{W^{1 / 2}}{\hat{k}^{2} \hat{\tau}_{e}}\left(1-\cos \left(\hat{k}^{3 / 2} \hat{t}\right)\right) .
$$

Assuming an impulsive acceleration, we expand this solution for $\tau_{e} \rightarrow 0$ and find for the amplitude and growth rate at the end of phase 1: $f_{1}\left(\hat{\tau}_{e}\right) \approx 1$ and $f_{1}^{\prime}\left(\hat{\tau}_{e}\right) \approx \mathrm{We}^{1 / 2} \hat{k}$. Each mode has therefore a specific growth rate as a result of the impulsive acceleration. However, the amplitude remains unchanged in phase 1 , which is a direct consequence of our assumption $1 / \omega_{1} \gg \tau_{e}$.

The Fourier modes in phase 2 and 3 are not directly amplified by an acceleration, that is $\hat{k}_{c, 2}=\hat{k}_{c, 3}=0$. In phase 2, the dispersion relation (7.17) reads then $\hat{\omega}_{2}^{2}=\hat{k}^{3}$ and the mode development is in view of Eq. (7.19) described by $f_{2}^{\prime \prime}=-\hat{k}^{3} f_{2}$. The initial conditions

$$
f_{2}(\hat{t}=0)=1 \text { and } f_{2}^{\prime}(\hat{t}=0)=\hat{k} \mathrm{We}^{1 / 2}
$$

match the second phase to the result obtained in the first phase. The solution is then a free harmonic oscillator

$$
f_{2}(\hat{t})=\cos \left(\hat{k}^{3 / 2} \hat{t}\right)+\frac{\mathrm{We}^{1 / 2}}{\hat{k}^{1 / 2}}+\sin \left(\hat{k}^{3 / 2} \hat{t}\right) .
$$

This solution for phase 2 is derived under the assumption of thick sheet, i.e. the dispersion relation characteristic of an infinite medium, i.e. $\hat{\omega}_{2}^{2}=\hat{k}^{3}$, was used. Consequently, as the drop expands into a thin sheet the solution (7.22) 
will inevitably be invalid at some point during the expansion when the two interfaces of the sheet are coupled by the Laplace pressure. This time defines the transition from the second to the third phase of the model and is most likely given by $\tau_{i}$, i.e. the timescale on which we observed the transition of the drop to a thin sheet in $\S 7.4$. We now assume a sheet expansion that is fast in comparison to the frequency of the harmonic oscillation described by Eq. (7.22), i.e. the sheet is thin at $\tau_{i}$ while all Fourier modes are still in their first oscillation. Comparing $\tau_{i}$ to the period of oscillation for each mode requires then $\hat{k}^{3 / 2} \mathrm{We}_{\mathrm{d}}^{-1 / 2} \ll 1$ for the assumption to be valid, which is justified for our experiments with $\mathrm{We}_{\mathrm{d}} \gg 1$. As a consequence, we expand the solution (7.22) in the limit $\hat{k}^{3 / 2} \hat{t} \rightarrow 0$ to obtain

$$
\begin{aligned}
& f_{2} \approx 1+\mathrm{We}^{1 / 2} \hat{k} \hat{t}-\frac{1}{2} \hat{k}^{3} \hat{t}^{2}, \\
& f_{2}^{\prime} \approx \mathrm{We}^{1 / 2} \hat{k}-\hat{k}^{3} \hat{t},
\end{aligned}
$$

which is used to determine the mode $\hat{k}_{\max }$ with the largest growth rate according to $\mathrm{d}\left(f_{2}^{\prime}\right) / \mathrm{d} k=0$. The result $\hat{k}_{\max }=\mathrm{We}^{1 / 4} /(3 \hat{t})^{1 / 2}$ is time dependent, since each mode resonates with its intrinsic frequency during the second phase. Our choice for the transition to the third phase of the model determines then the fastest growing mode $\hat{k}_{\max }\left(\tau_{i}\right)$ at the end of the second phase.

Phase 3 is characterized by the expansion of the sheet to $\hat{R} \gg 1$ until the amplitude of the fastest growing mode is of the order of the sheet thickness (Fig. $7.15(\mathrm{~d})$ ). We describe the mode development in the third phase by the thin-sheet dispersion relation, since we assume $k\left(t_{b}\right) h\left(t_{b}\right) \ll 1$. In absence of any acceleration the dispersion relation for a thin sheet is $\hat{\omega}_{3}=(k h)^{4} / 2$ [117] and when normalized in agreement with definition (7.16) we obtain $\hat{\omega}_{3}=$ $\hat{h} \hat{k}^{4} / 2$. In phase 1 and 2 , we neglected the effect of the sheet expansion on the dispersion relation of the modes. However, the characteristic of the third phase is just this expansion and simultaneous thinning of the sheet. We now take the effect of the sheet expansion on $\hat{\omega}_{3}$ into account by a self-similar stretch. Each quantity before the stretch, i.e. at the end of the second phase, is denoted by the subscript 0 such that the self-similar stretching reads $k_{0} R_{0}=k R$ and $\eta_{0} / k_{0}^{2} \sim \eta / k^{2}$. The former is the effect of the radial sheet expansion that stretches the wavelength of a given perturbation and the latter is the contraction of the perturbation amplitude resulting from mass conservation. Introducing the kinematics (7.10) of the sheet then leads to

$$
\hat{k}=\hat{k}_{0} \hat{R}^{-1} \text { and } \frac{\eta}{h}=\frac{\eta_{0}}{R_{0}} .
$$

Combining this self-similar stretching with the scaling laws (7.10) and (7.11) for the sheet kinematics we now recognize that the instantaneous growth 
rate during the expansion in phase 3 decays much faster then the sheet thins: $\hat{\omega}_{3} \sim \hat{t}^{-6}$ and $\hat{h} \sim \hat{t}^{-2}$. In conclusion, the sheet expansion and simultaneous thinning literally freezes the growth rate of the perturbation in its state at the end of the second phase, that is $f_{3}^{\prime \prime} \approx 0$ for $t>\tau_{i}$. As a result, the amplitude of the instability than evolves as

$$
f_{3}(\hat{t})=f_{2}\left(\hat{k}_{\max }, \hat{\tau}_{i}\right)+f_{2}^{\prime}\left(\hat{k}_{\max }, \hat{\tau}_{i}\right) \hat{t} \approx \mathrm{We}\left(\frac{E_{\mathrm{k}, \mathrm{d}}}{E_{\mathrm{k}, \mathrm{cm}}}\right)^{1 / 2} \hat{t},
$$

where we made use of $\hat{\tau}_{i}=\mathrm{We}_{\mathrm{d}}^{-1 / 2}$. We now acknowledge that the choice for $\tau_{i}$ as the characteristic time for the sheet thinning introduces the energy partition $E_{\mathrm{k}, \mathrm{d}} / E_{\mathrm{k}, \mathrm{cm}}$ for the first time in our analysis.

Following Bremond and Villermaux [117], the time of breakup $t_{b}$ is now found when the perturbation amplitude is of the order of the sheet thickness, that is

$$
\frac{\eta_{0}}{R_{0}} f_{3}\left(t_{b}\right)=1
$$

where we take into account the thinning (7.25). Once $t_{b}$ is known the wavenumber at breakup $\hat{k}_{b}$ is obtained by applying the stretching (7.25) to the result $\hat{k}_{\max }\left(\hat{\tau}_{i}\right)$ from the second phase. As a final result, we obtain as prediction for the sheet breakup

$$
\begin{aligned}
\frac{t_{b}}{\tau_{c}} & \sim\left(\frac{\eta_{0}}{R_{0}}\right)^{-1} \mathrm{We}^{-1}\left(\frac{E_{\mathrm{k}, \mathrm{d}}}{E_{\mathrm{k}, \mathrm{cm}}}\right)^{-1 / 2}, \\
k_{b} R_{0} & \sim \frac{\eta_{0}}{R_{0}} \operatorname{We}\left(\frac{E_{\mathrm{k}, \mathrm{d}}}{E_{\mathrm{k}, \mathrm{cm}}}\right)^{1 / 4} .
\end{aligned}
$$

These scaling laws for the breakup time $t_{b}$ and the characteristic wavenumber $k_{b}$ of hole nucleation are expressed in terms of the initial amplitude of the perturbation in relation to the drop size $\eta_{0} / R_{0}$, the Weber number We, and the energy partition $E_{\mathrm{k}, \mathrm{d}} / E_{\mathrm{k}, \mathrm{cm}}$. The stronger dependency of $t_{b}$ on We in comparison to $E_{\mathrm{k}, \mathrm{d}} / E_{\mathrm{k}, \mathrm{cm}}$ as expressed by the scaling exponents is evident for our experiments. We already recognized that Weber number affects both the initial acceleration and the sheet kinematics. It entered our model therefore already in the first phase when the growth rate of the Fourier modes are set. In addition, the Weber number also set the scaling for the kinematics (7.10) and (7.11) that we used in the third phase of our model. In contrast to the Weber number, the energy partition only influences the late-time evolution of the sheet. $E_{\mathrm{k}, \mathrm{d}} / E_{\mathrm{k}, \mathrm{cm}}$ enters therefore our model only in the third phase: it affects the characteristic time $\tau_{i}$, i.e. the time phase 3 is assumed to begin, and the expansion rate of the thin-sheet, which leads to the weaker scaling exponent for $E_{\mathrm{k}, \mathrm{d}} / E_{\mathrm{k}, \mathrm{cm}}$ in comparison to We. 
The comparison of prediction (7.28) with experimental data for MEK and tin is shown in Fig. 7.16. The breakup time $t_{b}$ is rescaled by $E_{\mathrm{k}, \mathrm{d}} / E_{\mathrm{k}, \mathrm{cm}}$ according to the prediction (7.28) to allow a direct comparison of MEK and tin as their kinetic-energy partition $E_{\mathrm{k}, \mathrm{d}} / E_{\mathrm{k}, \mathrm{cm}}$ differs. We find a good agreement for the scaling with the Weber number over three decades for the combined data of MEK and tin. We also show in Fig. 7.16 the time of destabilization in the neck region of MEK sheets. Also here, the experimental data and the prediction (7.28) agree in terms of the scaling with the Weber number. The different prefactor between the neck and center region can be explained by the radial thickness profile $h(r, t)$ : the neck region reaches the critical thickness $h_{\mathrm{b}}$ for a given Weber number at an earlier point in time compared to the center region. However, the kinematics of the neck and center region are described by the same scaling (7.10) and the development of the perturbation amplitude is assumed to be independent of the position on the sheet: a global Rayleigh-Taylor instability that is responsible for the breakup in the neck and center region alike. Consequently, the scaling exponent for the neck and center breakup are identical and in agreement with our prediction.

The prediction (7.29) for the characteristic wavenumber $k_{b}$ explains the large Weber number We $=30000$ in Fig. 7.14 that was required to recover the random hole nucleation by unbiased statistics. A strong acceleration as set by the Weber number affects the mode selection such that the wavelength of the fastest growing mode at the end of the second phase is small enough to be sampled experimentally during the breakup in the third phase. As a consequence, the scaling for the wavenumber $k_{b}$ cannot be validated experimentally for a sufficiently large range of Weber numbers. In both systems, it is a challegne to recover $k_{b}$ in unbiased statistics unless extremley large Weber numbers are reached.

The initial perturbation amplitude $\eta_{0} / R_{0}$ is a property that is assumed to be characteristic to each liquid system and independent of the wavenumber $k$. It is therefore equivalent to a white noise with a constant amplitude that is present during the acceleration phase of our model. This initial noise explains the early sheet breakup for MEK in comparison to the tin system in our analysis: the amplitude of perturbations during the early-time acceleration phase affects the destabilization at later times in the same way as the Weber number. The two liquid systems apparently exhibit different noise levels, which will be part of our discussion in the next section. 


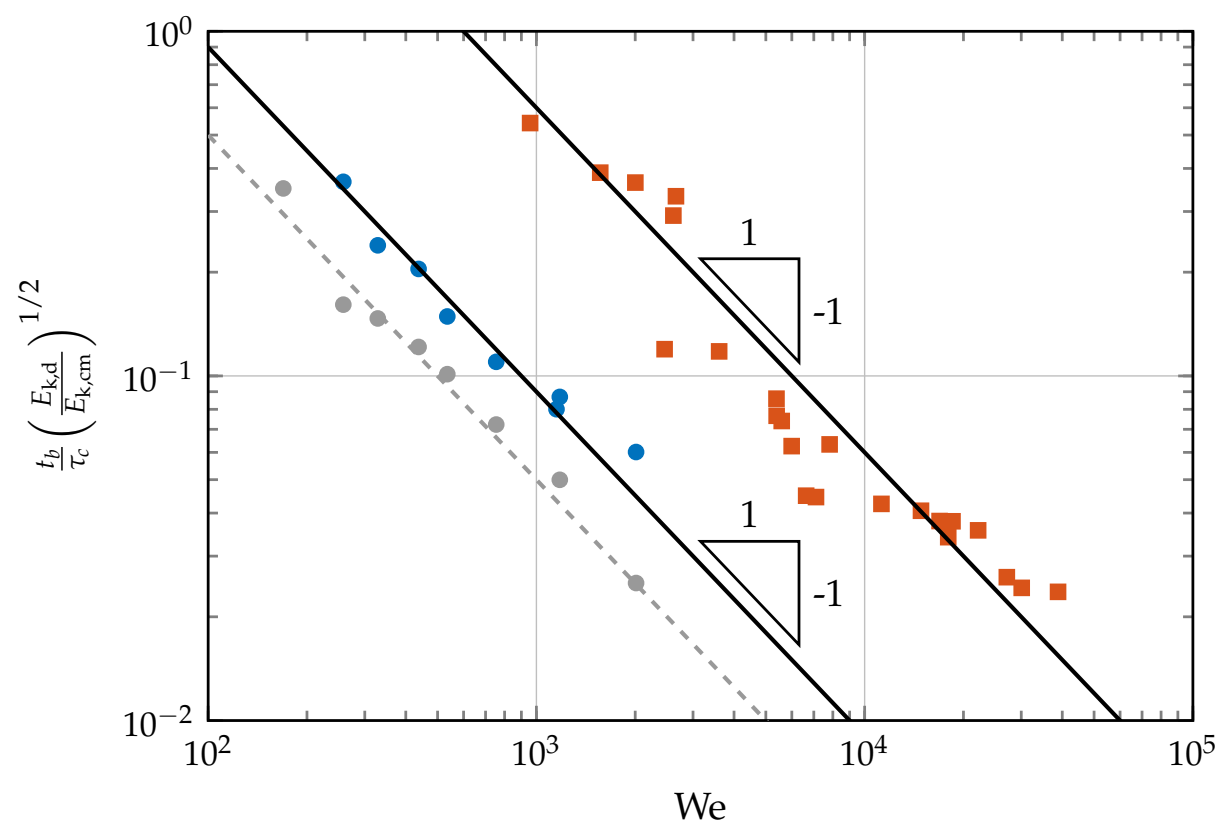

Figure 7.16: Breakup time $t_{b}$ for MEK $(\bullet)$ and tin drops ( $\square$ ) obtained for the sheet breakup in the center of the sheet. The experimental data points are obtained by finding the moment in time where the first hole in the center of a sheet $r / R \leq 0.5$ is observed. The solid lines (-) are the prediction (7.28) with $\eta_{0} / R_{0}=10^{-2}$ for MEK and $\eta_{0} / R_{0}=1.6 \times 10^{-3}$ for tin, both with a prefactor of 1 . For MEK the corresponding data for the neck region is shown in gray $(\bullet)$, which follows the same scaling law with $\eta_{0} / R_{0}=10^{-2}$ and prefactor of 0.5 .

\subsection{Discussion \& conclusion}

We study the fragmentation of a free-falling liquid drop that is propelled by a laser-induced phase change. We consider two liquid systems that differ in their propulsion mechanism: vapor-driven drops of methy ethyl ketone (MEK) and plasma-driven tin drops. The fragmentation shows the same features in both systems and is a late-time consequence of the initial drop acceleration that ultimately leads to the destabilization of the deforming liquid body. The deformation is characterized by a change of topology from the spherical drop into a flat sheet on the inertia timescale and a surface-tension limited expansion into a thin sheet on the capillary timescale. We identify two accelerations that are orthogonal to each other in the flat-sheet limit and amplify perturbations by Rayleigh-Taylor instabilities on the deforming liquid body. First, the impulsive acceleration of the drop surface by virtue of the laser impact causes a destabilization of the thin sheet, which we referred to as 
sheet breakup. The second acceleration is caused by capillary restoring forces: a rim develops at the edge of the expanding sheet and is subject to a local acceleration in the same way as it was described for the mechanical impact of drop on a pillar [42]. We referred to this type of destabilization as rim breakup. As the drop deformation and fragmentation are ultimately the consequence of the very same laser impact we find a signature of the laser-matter interaction in the fluid-dynamic response.

The plasma dynamics affects the expansion of the liquid sheet by how the kinetic energy is transferred to the drop at its surface. We describe this effect by a power-law scaling for the partition of kinetic energy into translation and deformation energy in relation to the laser energy. Once included in the description of the sheet kinematics, the rim breakup in both systems is entirely described in terms of the Weber number and the kinetic-energy partition. We then obtain scaling laws for the characteristic time and wavenumber of the rim breakup, which we validate by experimental data. Capillary restoring forces also cause a Rayleigh-Plateau instability during the rim breakup. Our analysis predicts the timescale of the Rayleigh-Plateau and Rayleigh-Taylor instability to be decoupled from the late-time expansion of the sheet for large Weber numbers, which is confirmed by experimental observations.

The Rayleigh-Taylor instability that leads to the sheet breakup already starts to develop during the initial drop acceleration by the laser-matter interaction. Consistently, our analysis of the sheet breakup leads to a third parameter, beside the Weber number and energy partition, that is necessary to distinguish between the different driving mechanisms: the amplitude of perturbations $\eta_{0} / R_{0}$ that are present during the initial acceleration of the drop. We trace the amplitude of these perturbations from the early-time acceleration to the late-time deformation of the drop. The evolution of the instability is described by the dispersion relation characteristic of each of the following three phases: the impulsive acceleration in the first phase is followed by an intermediate phase where the drop deforms into a flat sheet. Then follows a late-time phase where the expansion of the flat sheet into a thin sheet freezes any further development of the instability except for a self-similar stretching. We then obtain scaling laws for the characteristic time and wavenumber of the sheet breakup. The former is validated in both liquid systems by experimental data to find a good agreement between our model and the experiment.

We conclude from the time of sheet destabilization that the plasma-driven system exhibits an initial noise level of one order of magnitude lower than the vapor-driven system. The typical scale of $\eta_{0} / R_{0} \sim 10^{-2}$ in the vapor-driven system excludes the thermal noise as source for initial perturbations, since the thermal noise was found to be several orders of magnitude smaller in 
comparable systems [130]. We therefore attribute the difference in $\eta_{0} / R_{0}$ between the two systems to a macroscopic noise that originates in the different laser-matter interaction at the drop surface. The sheet breakup is then caused by a global Rayleigh-Taylor instability that leads to a destabilization in areas where the perturbation amplitude is of the order of the local sheet thickness. In both systems, a radial dependency is introduced in the sheet thickness by virtue of the topology change from a spherical drop to a flat sheet. In addition, azimuthal modulations in the initial laser radiation for vapor-driven drops translate to deterministic variations in the sheet thickness during the late-time expansion. As a consequence, likely areas of destabilization emerge on the sheet in azimuthal and radial direction during the fragmentation. The hole nucleation itself is found to be a randomly distributed process when observed on a length much smaller than the extent of the deterministic thickness variations.

The analysis of the fluid-dynamic instabilities in combination with experimental data leads to a phase diagram for the onset of fragmentation by laser-pulse impact (Fig. 7.17). At low Weber number, only the rim breakup is observed while the sheet remains stable. In this regime, the initial acceleration is not strong enough to amplify the initial perturbation sufficiently for the sheet breakup to occur. For increasing Weber number the rim destabilizes at earlier times before the maximum expansion of the sheet is reached. The trajectory of fragments that originate from the rim breakup is set by the sheet expansion rate at the moment of detachment, which leads to a cloud of small drops surrounding the sheet at maximum expansion. The sheet breakup occurs at two distinct regions that are set by the radial thickness profile during the late-time expansion: the neck and the center region of the sheet. Both regions follow the same scaling law for the characteristic time of destabilization, which is the consequence of a global Rayleigh-Taylor instability that is responsible for the breakup in the neck and center region alike.

Both the rim and sheet breakup lead to a structure of elongated ligaments: in case of the rim breakup, the ligaments point radially outward, whereas the sheet breakup leads to a two-dimensional web of ligaments. The parameter range where such a collection of ligaments is formed is marked as unstable domain $(\square)$ in Fig. 7.17. The last stage of the drop fragmentation is then characterized by the breakup of individual ligaments into stable fragments. The collection of all resulting fragments, which finally relax to a spherical shape, leads then to the overall size distribution of stable drops. The elementary size distribution coming from a single ligament breakup depends on the roughness and the size of the ligament $[42,49,52]$. Depending on the history of a ligament, i.e. the way it was created before its breakup, the elementary distribution is then more or less broad. Experimental data for the elementary 


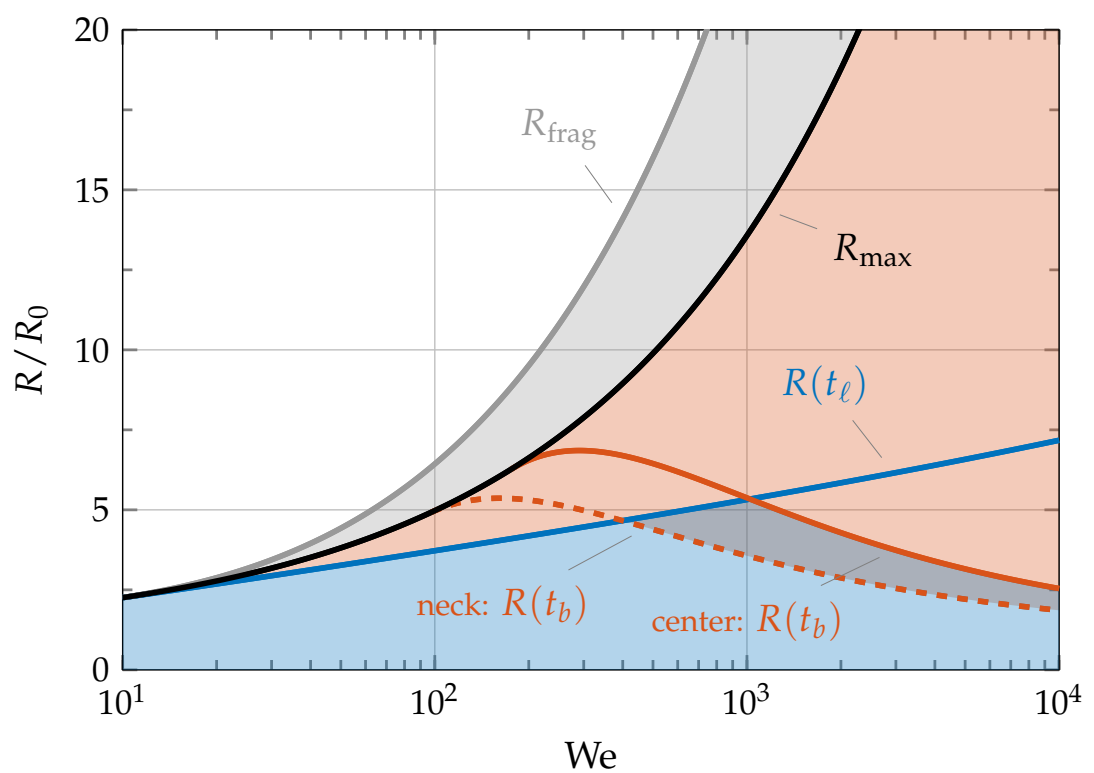

Figure 7.17: Overview on the stability of free-falling drops upon laser impact in terms of the Weber number and the radial expansion $R / R_{0}$. The parameter range of stable liquid sheets $(\square)$ is separated from the unstable domain $(\square)$ by the rim breakup at $R\left(t_{\ell}\right)$ and the sheet breakup at $R\left(t_{b}\right)$. The diagram is based on the MEK system for which the energy partition $E_{\mathrm{k}, \mathrm{d}} / E_{\mathrm{k}, \mathrm{cm}}=1.8$ and the initial perturbation amplitude $\eta_{0} / R_{0}=10^{-2}$ for the sheet breakup. The radii $R\left(t_{\ell}\right)$ and $R\left(t_{b}\right)$ follow then from the sheet kinematics (7.8) and the corresponding scaling (7.13) and (7.28) for the breakup time, where the sheet breakup $R\left(t_{b}\right)$ is given for the neck (- - - ) and center region (-). The prefactor in each scaling is obtained from the experimental data in Fig. 7.10 and Fig. 7.16, respectively. The maximum radius $R_{\max }(-)$ follows from the sheet kinematics (7.8) for $t_{\max } / \tau_{c}=2 / \sqrt{27}$. At the same time, the radius $R_{\text {frag }}$ $(-)$ is an estimate for the extent of the cloud of fragments $(\square)$ that originate from the rim breakup. The fragment trajectory is assumed to be set by the expansion rate at the moment of destabilization, i.e. $R_{\text {frag }}=R\left(t_{\ell}\right)+\dot{R}\left(t_{\ell}\right)\left(t_{\max }-t_{\ell}\right)$.

distribution after ligament breakup is best described by a gamma distribution [49]. The linear superposition of all elementary gamma distributions, one for each typical type of ligament, leads then to the overall size distribution of stable drops. The remaining problem is then to obtain the statistics for the typical ligaments that form in course of the rim and sheet breakup.

The final size distribution of the rim breakup for the mechanical impact of a drop an a pillar was successfully described by the linear combination of two elementary gamma distributions [42]. Each of the two distribution accounted for the breakup of a typical class of ligaments: one distribution for the radial 

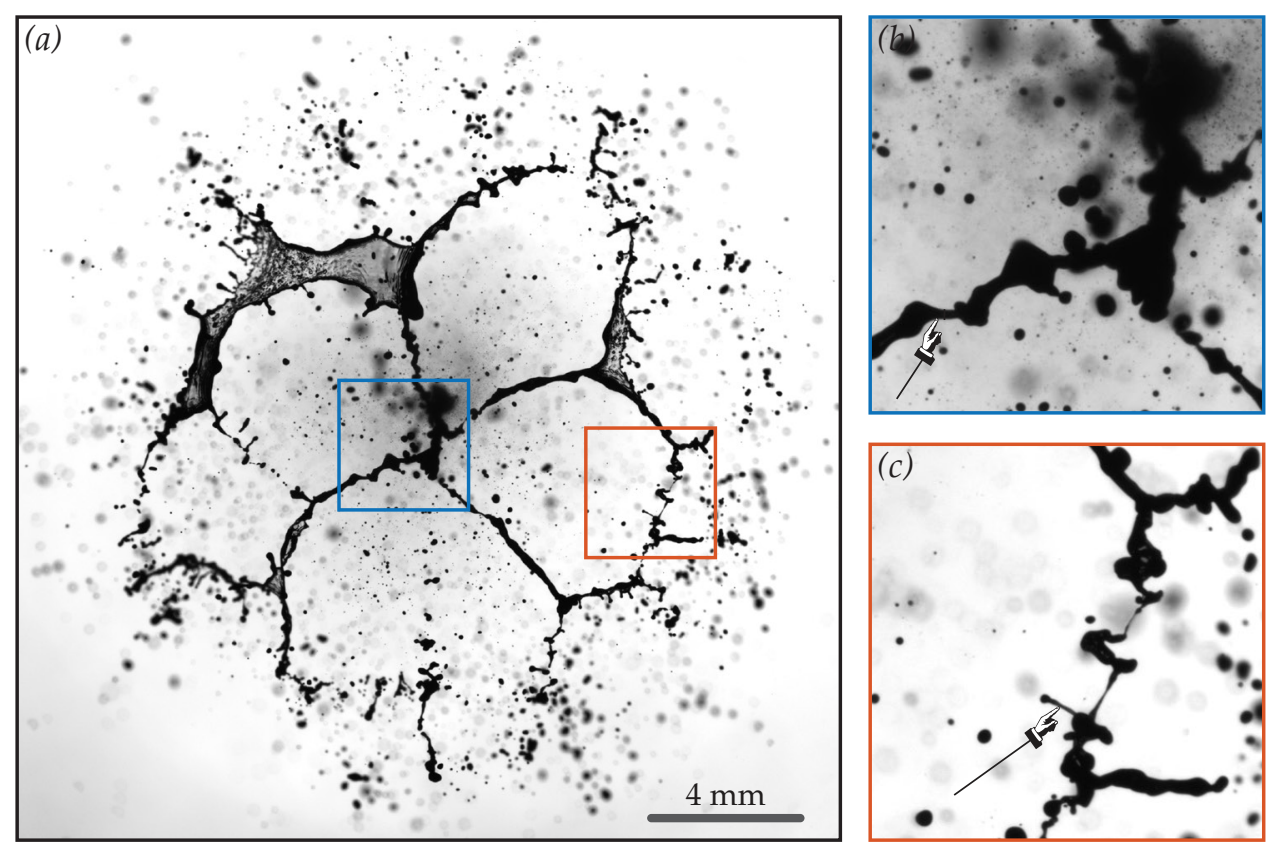

Figure 7.18: The web of ligaments for a MEK sheet at We $=750$ in (a) and two magnified parts from the center (b) and neck region (c) of the sheet. The typical diameter of ligaments $d_{\ell}$ varies by more than one order of magnitude as indicated by the two pointers in $(b, c)$ that mark ligaments with approximately $d_{\ell}=100 \mu \mathrm{m}$ and $d_{\ell}=4 \mu \mathrm{m}$.

ligaments and a second one for the remnant rim not completely converted into radial ligaments. As pointed out by Villermaux and Bossa [42] the experimental data for the size distribution was obtained under very symmetric impact conditions. In the currrent study, the high degree of symmetry observed in the tin system, especially the clear observation of the characteristic wavenumber $k_{\ell}$, leads to the expectation that a bimodal distribution is sufficient as well to describe the rim breakup. In contrast to the tin system, the rim breakup in the MEK system is affected by the sheet breakup, which already limited the amount of data we could obtain for the rim breakup in that system. In turn, this interaction of the rim and the sheet may also require more than two elementary distributions to capture the rim breakup in the MEK system.

The statistics for the fragmentation of a web of ligaments has been described in detail by Lhuissier and Villermaux [52]. In this study, a planar Savart sheet is seeded with small air bubbles to cause a random hole piercing on the sheet. The growth and merging of holes in this experiment lead then to a web of ligaments, similar to the one observed in our study. The 
fragmentation of the ligament structure leads to a broad size distribution of fragments that was almost fully recovered by the elementary distribution for a single type of ligaments [52]. Since the ligaments are strongly corrugated by large-amplitude random capillary waves, even a single elementary distribution leads to a broad collection of drop sizes. Other contribution, e.g. drops originating from the nodes of the web of ligaments, lead then to a slightly broader final size distribution. A possible source of very small drops that was observed by Lhuissier and Villermaux [52] but lay outside the parameter domain is a secondary sheet formation due to the inertia of colliding rims: if the hole-opening speed is large the colliding rims do not merge but splash, forming a secondary sheet that is oriented orthogonal to the original one. We observe a similar process in our experiments, in particular for rims that originate from a hole nucleation in the neck region where the sheet is thinnest and the hole-opening speed the largest: the rims collide in an asymmetric fashion and form highly corrugated ligaments or even splash to first form a collection of thinner ligaments before they finally break into stable drops. The manifestation of such a collection of ligaments can be seen in Fig. 7.18 for a single MEK sheet. We observe a variation in the typical size of ligaments by more than order of magnitude. Furthermore, the inset Fig. 7.18 (c) reveals the highly-corrugated cylindrical geometry of ligaments. We now recognize the necessity to consider more than one elementary size distribution to capture this broad size distribution already observed for the ligaments themselves.

Given the aforementioned considerations the final size distribution of the laser impact on a drop is expected to be broad distribution as a result of multiple elementary size distributions. The experimental investigation for both systems in terms of the size distribution is a challenge. On the one hand, the optical resolution of the shadowgraph images limits the range of fragment sizes that can be detected in the tin system. The small ligaments in Fig. 7.18 (c) for the MEK system suggest a typical size of $d_{\ell} / R_{0} \sim 10^{-2}$, which is in absolute terms then a few hundred nanometer for the tin system, i.e. far below the resolution of optical imaging in the visible spectrum. Measurement techniques based on light scattering seem to be a viable approach, but require suitable calibration particles to measure reliable size distributions, which are difficult to obtain for metals [131]. On the other hand, the MEK system shows large-scale facets that are set by the laser-beam profile (compare Fig. 7.13) and not of universal nature. These influences need to be incorporated in an statistical analysis in a transparent way to obtain a description that holds for both the tin and MEK system. Such an analysis clearly deserves further investigation, where the work presented here is the first step towards a full description of the drop fragmentation that incorporates the chaotic and deterministic facets of the ligaments formation. 


\section{A Rescaling the late-time sheet model}

The impact of a drop with the velocity $U$ and initial radius $R_{0}$ on a pillar of the same size leads to the development of a sheet away from the pillar with thickness $h(r, t)$ and radius $R(t)$ with dynamics [42]

$$
\begin{aligned}
\frac{R(t)-R_{0}}{R_{0}} & =\sqrt{\mathrm{We}_{\mathrm{d}}} \frac{t}{\tau_{c}}\left(1-\frac{\sqrt{3}}{2} \frac{t}{\tau_{c}}\right)^{2}, \\
h(r, t) & \sim \frac{R_{0}^{3}}{U r t}=\frac{R_{0}^{2} \tau_{c}}{\sqrt{\mathrm{We}_{\mathrm{d}}} r t^{\prime}} \\
u_{r}(r, t) & =\bar{t}^{\prime}
\end{aligned}
$$

where $u_{r}$ is the radial velocity inside the sheet. Here, we introduced the rescaled Weber number $\mathrm{We}_{\mathrm{d}}$ to account for different impact conditions in the context of a laser impact in comparison to the mechanical impact on a pillar.

We now seek the relation between the Weber number We $=\rho U^{2} R_{0} / \gamma$, $\mathrm{We}_{\mathrm{d}}$ and the energy partition

$$
\frac{E_{\mathrm{k}, \mathrm{d}}}{E_{\mathrm{k}, \mathrm{cm}}}=\frac{\int_{R_{0}}^{R} u_{r}^{2} h r \mathrm{~d} r}{U^{2} \int_{R_{0}}^{R} h r \mathrm{~d} r}=\frac{R^{3}-R_{0}^{3}}{3 U^{2} t^{2}\left(R-R_{0}\right)},
$$

where we choose $R_{0}$ as lower integration limit to avoid the singularity in $h(r, t)$ for $r \rightarrow 0$. We evaluate expression (7.33) at the time $\tau_{i}$, i.e. when the topology of the spherical drop already changed to a thin sheet. We make us of $\left(R-R_{0}\right) \approx R_{0} \sqrt{\mathrm{We}_{\mathrm{d}}} \tau_{i} / \tau_{c}$ and then $\left(R^{3}-R_{0}^{3}\right) / \tau_{i}^{3} \approx R_{0}^{3} \mathrm{We}_{\mathrm{d}}^{3 / 2} / \tau_{c}^{3}$, which is valid for $W_{\mathrm{d}} \gg 1$ and $t=\tau_{i}$. The center-of-mass speed $U$ in $E_{\mathrm{k}, \mathrm{cm}}$ is set by the Weber number such that $U^{2}=W e R_{0}^{2} / \tau_{c}^{2}$ in Eq. (7.33) leading to

$$
\mathrm{We}_{\mathrm{d}}=3 \mathrm{We} \frac{E_{\mathrm{k}, \mathrm{d}}}{E_{\mathrm{k}, \mathrm{cm}}} \text {. }
$$

This results explains our rescaling in Eq. (7.8), where we introduce $\mathrm{We}_{\mathrm{d}}$ in accordance with our previous definition of $E_{\mathrm{k}, \mathrm{d}} / E_{\mathrm{k}, \mathrm{cm}}$ in Eq. (7.7). We then use the result of the early-time model $E_{\mathrm{k}, \mathrm{d}} / E_{\mathrm{k}, \mathrm{cm}} \approx 1.8$ as explained in $\S 6$, which effectively initializes the late-time model with an expansion rate as predicted by the early-time model for the flat-top beam profile used in the MEK system. The near-field imaging and beam-shaping technique, which we explain in $\S 3$, is applied in this study only to improve the axi-symmetry of the beam such that $E_{\mathrm{k}, \mathrm{d}} / E_{\mathrm{k}, \mathrm{cm}}$ remains unchanged. 
158 CHAPTER 7. DROP FRAGMENTATION BY LASER-PULSE IMPACT 


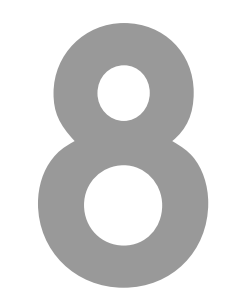

\section{Summary and Outlook}

Light moves liquid matter in various ways. Photons exchange momentum with a liquid sample as the light path changes at the interface between two media of different refractive index, an effect described as optical radiation pressure [57]. Intense field strengths are required to exert the radiation pressure onto the liquid body, and therefore focused laser light is often used. Laser radiation can also induce thermocapillary forces: the localized heating by a laser, either directly by linear absorption of the light in the liquid sample or indirectly by heating the substrate the liquid sample is in contact with, introduces thermocapillary stresses $[60,61]$ that drive the fluid dynamics. This effect of induced fluid motion by thermocapillary stresses is well-known as the Marangoni effect [62]. A laser-induced phase change is another way to move liquids by optical radiation, which allows for large deformations and flow speeds to be reached, see e.g. Refs. [26-28, 33].

In this thesis we studied how a liquid drop responds to the impact of a laser pulse that induces a phase change concentrated both in time and space, i.e. localized to the drop surface and much shorter than the response of the drop. The response was found to be violent as illustrated in $\S 2$ : the drop is accelerated, strongly deforms, and eventually fragments. Shock waves, the ejection of matter, and even plasma generation can accompany this process. The goal of this thesis was to describe and understand the life of the drop: starting with the laser impact, followed by the deformation of the drop and how it depends on the laser-pulse properties up to the final breakup of the liquid body into tiny fragments. 
An integral part of this thesis was the design, planning, and construction of an experimental setup as presented in $\S 3$ to control and visualize the fluid-dynamic response of a liquid drop to a laser-induced phase change. We made use of a laser system that emits nanosecond, high-energetic pulses of green light. The uniformity of the laser radiation was improved and even controlled by a beam-shaping and near-field imaging technique before the impact on the drop. A millimeter-sized drop of initial radius $R_{0}$ was generated from different liquid-dye solutions that we characterized in terms of their absorption characteristics for green light. The response of the drop to the laser impact was visualized by high-speed and stroboscopic high-resolution imaging in two orthogonal views: a side-view perpendicular to the laser beam and a back-view that was along the laser-beam propagation. The combination of imaging techniques with exposure times down to a few nanoseconds resolved all relevant timescales that vary by orders of magnitude between the nanosecond duration of the laser impact and the millisecond evolution of the fluid-dynamic response. The setup enabled an operation at constant control parameters with a shot-to-shot variation of less than 3\%, which made stroboscopic imaging possible in the first place. Having developed an experimental setup that allowed to visualize the impact condition of the drop as well as its fluid-dynamic response, we explored the life of the drop upon laser impact as explained in $\S 4$ to $\S 7$.

In $\S 4$ we focused on the question how the laser pulse transfers momentum to a liquid body that absorbs the laser energy. To this end, we limited the laser-matter interaction to a thin, superficial layer of the drop. The thickness of this layer is set by the amount of dye dissolved in the liquid and the absorption characteristics of the liquid-dye solution at the laser wavelength. We showed that the key driving mechanism for the fluid motion is the local and asymmetric boiling of the liquid on the illuminated side of the drop. The deposited energy per unit mass in the superficial layer is comparable to the specific latent heat of vaporization. As a result this layer boils and a shock wave is emitted in the surrounding air, followed by the directive emission of vapor and mist. This expelled mass is only a tiny fraction of the total mass of the drop but results in a recoil pressure on the drop surface. As a consequence, the drop is impulsively accelerated as a whole, where the center-of-mass velocity $U$ is proportional to the laser-pulse energy once the threshold energy that is needed to heat the liquid layer to the boiling point is reached.

The fluid motion can also be induced by plasma generation as studied in $\S 5$ in collaboration with the Advanced Research Center for Nanolithography (ARCNL). We demonstrated that a metal drop can be propelled by an expanding plasma cloud in the vicinity of the drop surface. The drop is placed in the focus point of a laser beam where the local fluence exceeds the limit for 
plasma generation. As soon as a plasma is generated within a fraction of the laser-pulse duration, inverse-bremsstrahlung absorption strongly decreases the initially high reflectivity of the metallic surface to negligible values. Any further laser radiation is then absorbed by the plasma cloud that exerts a pressure on the drop surface accelerating the drop as a whole. Therefore, the description of the drop propulsion by a short recoil pressure due to a forceful ejection of matter is analogous to the vapor-driven drop. We found remarkable one-to-one correspondences between the two propulsion mechanisms, including the description of an analogous onset effect, even though the physical origins of the propulsion are completely different.

We recognized the clear separation of timescales characteristic to both vapor-driven and plasma-driven drops. The laser-matter interaction takes place on a nanosecond timescale set by the laser-pulse duration $\tau_{p}$ and accelerates the drop on the timescale $\tau_{e}$ during which the ejection of matter occurs. The drop propulsion is accompanied by a lateral expansion that occurs on the inertia timescale $\tau_{i}=R_{0} / U$ and is eventually slowed down by surface tension $\gamma$ on the capillary timescale $\tau_{c}=\left(\rho R_{0}^{3} / \gamma\right)^{1 / 2}$. The clear separation of timescales then reads

$$
\tau_{e} \ll \tau_{i}<\tau_{c},
$$

which formed the basis for our understanding of the laser impact on a freefalling drop: the laser impact induces a phase change that results in an impulsive acceleration of the liquid interface on the timescale $\tau_{e}$ that is much shorter than the subsequent fluid-dynamic response on the timescale $\tau_{i}$ and $\tau_{c}$.

The separation of timescales allowed us to treat the laser impact on a drop in a general way, independent of the actual driving mechanism: the interaction of a laser pulse with a liquid drop can successfully be modeled by applying a recoil-pressure pulse to the drop surface as we showed in $\S 6$. The deformation of the drop can then be described in three stages. An early-time stage where the spherical drop is accelerated and gains kinetic energy as a result of a recoil pressure acting on its surface. In the subsequent stage the drop deforms into a sheet, and a late-time stage where the sheet undergoes a surface-tension limited expansion. An analytical model for the early-time stage when the drop is still spherical provided the drop dynamics as a function of the laserpulse shape: the kinetic-energy partition inside the drop was obtained, from which we derived the amount of deformation versus translation of the drop. We found that, for a given propulsion of the drop, a maximal expansion is obtained when the laser pulse is focused into a tight spot, which results in a strongly curved sheet. By contrast, a flat symmetric sheet can only be obtained with a uniform laser-beam profile. On the inertial and capillary timescales boundary-integral simulations, validated in one-to-one comparison 
to experiments, revealed the details of the sheet thickness and curvature.

We also sought an analytical description of the drop deformation during the late-time stage, since it is of crucial importance for the analysis of the drop fragmentation. Assuming a flat drop, we derived an analytical thin-sheet model to describe the expansion and recoil dynamics as controlled by capillary restoring forces. The model approach is based on the separation of timescales and allows to incorporate other models that are derived under the same premise, such as a sheet model that was originally derived for the mechanical impact of a drop on a pillar [42]. Each late-time model was initialized by the kinetic-energy partition that is set by the recoil-pressure pulse on the drop surface during the early-time stage. The spatial distribution of the pressure pulse can be considered to follow that of the laser radiation at the drop surface in case of vapor-driven drops. As such, the profile was accessible for a direct optical measurement in our experimental setup. By contrast, the profile for plasma-driven drops was found to depend on the plasma dynamics during the laser impact. We described this effect by a power-law scaling for the kineticenergy partition in terms of the laser energy. Once this relation was known, the models for the late-time sheet kinematics captured the characteristics of the laser-matter interaction and could be used in a universal way for both vapor-driven and plasma-driven drops.

With a description of the deformation at hand, we studied the fragmentation of the drop as summarized in $\S 7$. We identified two accelerations that are orthogonal to each other and amplify perturbations by Rayleigh-Taylor instabilities on the deforming liquid body. First, the impulsive acceleration of the drop surface by virtue of the laser impact causes a destabilization of the thin sheet during the late-time stage. The second acceleration is caused by capillary restoring forces and leads to a destabilization of the edge of the sheet. In our model, we traced the amplitude of surface perturbations from the earlytime acceleration to the late-time deformation of the drop to obtain scaling laws for the characteristic time and wavenumber of the breakup. We found a good agreement between our model and experimental data. As the drop deformation and fragmentation are ultimately the consequence of the very same laser impact we found a signature of the laser-matter interaction in the late-time fragmentation. We concluded that the initial perturbation amplitude is set by a macroscopic noise that originates in the laser-matter interaction at the drop surface. The breakup of the sheet is then caused by a global RayleighTaylor instability that leads to a destabilization in areas where the perturbation amplitude is of the order of the local sheet thickness. A radial dependency is introduced in the sheet thickness by virtue of the topology change from a spherical drop to a flat sheet. In addition, azimuthal modulations in the initial laser radiation for vapor-driven drops translate to deterministic variations in 
the sheet thickness during the late-time expansion. As a consequence, likely areas of destabilization emerge on the sheet in azimuthal and radial direction during the fragmentation. The nucleation of holes on the liquid sheet was found to be a randomly distributed process when observed on a length much smaller than the extent of the deterministic thickness variations.

In summary, we described the life of a drop that starts with a laser impact concentrated both in time and space and leads to a fluid-dynamic response. In particular, we considered a laser-induced vaporization at the drop surface and a laser-produced plasma cloud in the vicinity of the drop as driving force for the fluid dynamics. The response of the drop can be summarized as a characteristic change of the liquid topology: the spherical drop deforms into a liquid sheet that expands radially under the influence of surface tension and finally fragments. We provided analytical descriptions for the kinematics of the deformation that we validated extensively. In addition, we demonstrated that the interaction of a laser pulse with a liquid body can successfully be modeled by applying a recoil-pressure pulse to the liquid interface. The details of the laser-matter interaction are then described by an appropriate formulation of the pressure profile. The drop fragmentation is caused by Rayleigh-Taylor instabilities that destabilize the deforming liquid body, for which we derived scaling laws in terms of the characteristic time and wavenumber of breakup.

A major step to characterize the drop fragmentation upon laser impact was done in this thesis: the physical cause of destabilization was identified and it was shown how it is affected by the laser-matter interaction. However, further investigations are required to obtain a transparent description for the size distribution of fragments. On the one hand, experimental effort is required to measure the size distribution for the micrometer-sized, plasma-driven drops. On the other hand, the two-dimensional structure of liquid ligaments that emerges during the fragmentation needs to be described in a transparent way, that is a formal description that incorporates the chaotic and deterministic facets of the ligament formation.

The experimental setup described in this thesis can be used in future research to study laser-matter interactions and laser-induced liquid motion for a broader range of experiments: the target, in our case a free-falling spherical drop, may be replaced with planar geometry such as a liquid film to study the laser-induced forward motion, for example for non-Newtonian liquids or viscoelastic hydrogels. In general, the laser impact upon a liquid target results in a forcing, concentrated both in time and space, and can be visualized in our setup. This allows for accurate and quantitative studies of laser-matter interactions that induce fluid motion.

The separation of timescales (8.1) allowed us to decouple the fluid-dynamic response from the details of their driving force. Our analysis of both the 
deformation and fragmentation made use of this principle. At the same time we can indicate possible directions for future work that is required in case this clear separation is not valid anymore. The first limitation is given when the timescale of acceleration $\tau_{e}$ is of the order of the inertia timescale $\tau_{i}$ or even longer. This case can be explored experimentally by making use of laser systems with longer pulse durations than used in this work. The liquid interface is then accelerated while the deformation takes place. This regime, although challenging for an analytical treatment, has practical relevance as the same kinetic energy can be supplied to the liquid body while the peak power of laser radiation is reduced.

A timescale which was not of great importance in this thesis is the acoustic timescale $R_{0} / c$, which is the time for the recoil pressure to be transmitted through the drop with the speed of sound $c$. In our experiments $\tau_{e}>R_{0} / c$ such that the pressure information is communicated inside the drop instantaneously in comparison to the duration of the acceleration. As a consequence, our treatment of the liquid phase as incompressible was valid. However, we already attributed early jetting phenomena in our experiments as described in $\S 7.4 .1$ to compressibility effects, while the influence on our results was found to be negligible. Much more violent experimental observations of compressibility effects, such as cavitation inside liquid drops, were published recently [132] and show a different fluid-dynamic response than described in this thesis. First analytical work in that regime is currently being made [122]. The regime of $\tau_{e}<R_{0} / c$ is also of increasing practical relevance in the near future, since laser systems with pulse duration far below a few nanoseconds are available and allow for driving forces of much shorter duration than applied in this thesis. 


\section{References}

[1] O. Ellabban, H. Abu-Rub, and F. Blaabjerg. Renewable energy resources: Current status, future prospects and their enabling technology. Renewable and Sustainable Energy Reviews, 39:748-764, 2014.

[2] I. H. G. S. Consortium and others. Finishing the euchromatic sequence of the human genome. Nature, 431(7011):931-945, 2004.

[3] C. M. Soukoulis and M. Wegener. Past achievements and future challenges in the development of three-dimensional photonic metamaterials. Nature Photonics, 5(9):523-530, 2011.

[4] G. M. Harry and the LIGO Scientific Collaboration. Advanced LIGO: The next generation of gravitational wave detectors. Classical and Quantum Gravity, 27 (8):084006, 2010.

[5] G. F. Giudice. A Zeptospace Odyssey: A Journey into the Physics of the LHC. Oxford University Press, Oxford; New York, 2010.

[6] C. Freeman and F. Louçã. As Time Goes By: From the Industrial Revolutions to the Information Revolution. Oxford University Press, Oxford, 2002.

[7] M. Castells. The Rise of the Network Society. Number 1 in The information age: economy, society, and culture. Wiley-Blackwell, 2010.

[8] M. Hilbert and P. López. The world's technological capacity to store, communicate, and compute information. Science, 332(6025):60-65, 2011.

[9] G. Moore. Cramming More Components Onto Integrated Circuits. Proceedings of the IEEE, 86(1):82-85, 1998.

[10] C. Wagner and N. Harned. EUV lithography: Lithography gets extreme. Nature Photonics, 4:24-26, 2010.

[11] J. Benschop, V. Banine, S. Lok, and E. Loopstra. Extreme ultraviolet lithography: Status and prospects. Journal of Vacuum Science \& Technology B: Microelectronics and Nanometer Structures, 26(6):2204-2207, 2008.

[12] E. R. Pennell and J. Pennell. Lithography and Lithographers; Some Chapters in the History of the Art, by Elizabeth Robins Pennell, Together with Descriptions and Technical Explanations of Modern Artistic Methods, by Joseph Pennell. The Macmillan Company, New York, 1915. 
[13] J. R. Sheats and B. W. Smith, editors. Microlithography: Science and Technology. Marcel Dekker, New York, 1998.

[14] B. Wu and A. Kumar. Extreme ultraviolet lithography: A review. Journal of Vacuum Science \& Technology B: Microelectronics and Nanometer Structures, 25(6): 1743-1761, 2007.

[15] V. Y. Banine, K. N. Koshelev, and G. H. P. M. Swinkels. Physical processes in EUV sources for microlithography. Journal of Physics D: Applied Physics, 44(25): 253001, 2011.

[16] S. Fujioka, M. Shimomura, Y. Shimada, S. Maeda, H. Sakaguchi, Y. Nakai, T. Aota, H. Nishimura, N. Ozaki, A. Sunahara, K. Nishihara, N. Miyanaga, Y. Izawa, and K. Mima. Pure-tin microdroplets irradiated with double laser pulses for efficient and minimum-mass extreme-ultraviolet light source production. Applied Physics Letters, 92(24):241502, 2008.

[17] H. Mizoguchi, T. Abe, Y. Watanabe, T. Ishihara, T. Ohta, T. Hori, A. Kurosu, H. Komori, K. Kakizaki, A. Sumitani, O. Wakabayashi, H. Nakarai, J. Fujimoto, and A. Endo. First generation laser-produced plasma source system for HVM EUV lithography. Proceedings SPIE, 7636:763608, 2010.

[18] G. O'Sullivan, B. Li, R. D'Arcy, P. Dunne, P. Hayden, D. Kilbane, Tom McCormack, H. Ohashi, F. O'Reilly, P. Sheridan, E. Sokell, C. Suzuki, and Takeshi Higashiguchi. Spectroscopy of highly charged ions and its relevance to EUV and soft x-ray source development. Journal of Physics B: Atomic, Molecular and Optical Physics, 48(14):144025, 2015.

[19] K. Tomita, Y. Sato, K. Nishikawa, K. Uchino, T. Yanagida, H. Tomuro, Y. Wada, M. Kunishima, T. Kodama, H. Mizoguchi, and A. Sunahara. Development of a collective Thomson scattering system for laser-produced tin plasmas for extreme-ultraviolet light sources. Applied Physics Express, 8:126101, 2015.

[20] A. Vogel and V. Venugopalan. Mechanisms of Pulsed Laser Ablation of Biological Tissues. Chemical Reviews, 103(2):577-644, 2003.

[21] C. W. Visser, R. Pohl, C. Sun, G.-W. Römer, B. Huis in 't Veld, and D. Lohse. Toward 3D Printing of Pure Metals by Laser-Induced Forward Transfer. Advanced Materials, 27(27):4087-4092, 2015.

[22] M. Merchant and S. R. Weinberger. Recent advancements in surface-enhanced laser desorption/ionization-time of flight-mass spectrometry. Electrophoresis, 21 (6):1164-1177, 2000.

[23] J. L. Norris and R. M. Caprioli. Analysis of Tissue Specimens by Matrix-Assisted Laser Desorption/Ionization Imaging Mass Spectrometry in Biological and Clinical Research. Chemical Reviews, 113(4):2309-2342, 2013.

[24] R. Eason, editor. Pulsed Laser Deposition of Thin Films: Applications-Led Growth of Functional Materials. Wiley-Interscience, Hoboken, New Jersey, 2007. 
[25] F.-X. Rong. Liquid target pulsed laser deposition. Applied Physics Letters, 67(7): 1022-1024, 1995.

[26] R. G. Pinnick, A. Biswas, R. L. Armstrong, S. G. Jennings, J. D. Pendleton, and G. Fernández. Micron-sized droplets irradiated with a pulsed $\mathrm{CO}_{2}$ laser: Measurement of explosion and breakdown thresholds. Applied Optics, 29(7): 918-925, 1990.

[27] P. Kafalas and J. Herrmann. Dynamics and Energetics of the Explosive Vaporization of Fog Droplets by a 10.6- $\mu \mathrm{m}$ Laser Pulse. Applied Optics, 12(4):772-775, 1973.

[28] P. Kafalas and A. P. Ferdinand. Fog Droplet Vaporization and Fragmentation by a 10.6- $\mu \mathrm{m}$ Laser Pulse. Applied Optics, 12(1):29-33, 1973.

[29] C. Favre, V. Boutou, S. Hill, W. Zimmer, M. Krenz, H. Lambrecht, J. Yu, R. Chang, L. Woeste, and J.-P. Wolf. White-Light Nanosource with Directional Emission. Physical Review Letters, 89(3):035002, 2002.

[30] A. Lindinger, J. Hagen, L. D. Socaciu, T. M. Bernhardt, L. Wöste, D. Duft, and T. Leisner. Time-resolved explosion dynamics of $\mathrm{H}_{2} \mathrm{O}$ droplets induced by femtosecond laser pulses. Applied Optics, 43(27):5263-5269, 2004.

[31] Y. E. Geints, A. M. Kabanov, G. G. Matvienko, V. K. Oshlakov, A. A. Zemlyanov, S. S. Golik, and O. A. Bukin. Broadband emission spectrum dynamics of large water droplets exposed to intense ultrashort laser radiation. Optics letters, 35 (16):2717-2719, 2010.

[32] J.-Z. Zhang, J. K. Lam, C. F. Wood, B.-T. Chu, and R. K. Chang. Explosive vaporization of a large transparent droplet irradiated by a high intensity laser. Applied Optics, 26(22):4731-4737, 1987.

[33] Y. Tagawa, N. Oudalov, C. W. Visser, I. R. Peters, D. van der Meer, C. Sun, A. Prosperetti, and D. Lohse. Highly Focused Supersonic Microjets. Physical Review X, 2(3):031002, 2012.

[34] C. Sun, E. Can, R. Dijkink, D. Lohse, and A. Prosperetti. Growth and collapse of a vapour bubble in a microtube: The role of thermal effects. Journal of Fluid Mechanics, 632:5-16, 2009.

[35] A. Vogel, S. Busch, and U. Parlitz. Shock wave emission and cavitation bubble generation by picosecond and nanosecond optical breakdown in water. The Journal of the Acoustical Society of America, 100(1):148-165, 1996.

[36] S. T. Thoroddsen, K. Takehara, T. G. Etoh, and C.-D. Ohl. Spray and microjets produced by focusing a laser pulse into a hemispherical drop. Physics of Fluids, 21(11):112101, 2009.

[37] I. Apitz and A. Vogel. Material ejection in nanosecond Er:YAG laser ablation of water, liver, and skin. Applied Physics A, 81(2):329-338, 2005. 
[38] V. Horneffer, N. Linz, and A. Vogel. Principles of laser-induced separation and transport of living cells. Journal of Biomedical Optics, 12(5):054016, 2007.

[39] R. R. Gattass and E. Mazur. Femtosecond laser micromachining in transparent materials. Nature photonics, 2(4):219-225, 2008.

[40] P. K. Kundu, I. M. Cohen, D. R. Dowling, and G. Tryggvason. Fluid Mechanics. Elsevier/AP, Amsterdam; Boston, sixth edition, 2016.

[41] W. Schröder. Fluidmechanik. Number 7 in Aachener Beiträge zur Strömungsmechanik. Wissenschaftsverlag Mainz, Aachen, 2004.

[42] E. Villermaux and B. Bossa. Drop fragmentation on impact. Journal of Fluid Mechanics, 668:412-435, 2011.

[43] T. Tran, H. J. J. Staat, A. Susarrey-Arce, T. C. Foertsch, A. van Houselt, H. J. G. E. Gardeniers, A. Prosperetti, D. Lohse, and C. Sun. Droplet impact on superheated micro-structured surfaces. Soft Matter, 9(12):3272-3282, 2013.

[44] C. Clanet, C. Béguin, D. Richard, and D. Quéré. Maximal deformation of an impacting drop. Journal of Fluid Mechanics, 517:199-208, 2004.

[45] A. Yarin. DROP IMPACT DYNAMICS: Splashing, Spreading, Receding, Bouncing.... Annual Review of Fluid Mechanics, 38(1):159-192, 2006.

[46] P. Tsai, M. H. W. Hendrix, R. R. M. Dijkstra, L. Shui, and D. Lohse. Microscopic structure influencing macroscopic splash at high Weber number. Soft Matter, 7 (24):11325-11333, 2011.

[47] G. Riboux and J. M. Gordillo. Experiments of Drops Impacting a Smooth Solid Surface: A Model of the Critical Impact Speed for Drop Splashing. Physical Review Letters, 113(2):024507, 2014.

[48] C. W. Visser, P. E. Frommhold, S. Wildeman, R. Mettin, D. Lohse, and C. Sun. Dynamics of high-speed micro-drop impact: Numerical simulations and experiments at frame-to-frame times below 100 ns. Soft Matter, 11(9):1708-1722, 2015.

[49] E. Villermaux. Fragmentation. Annual Review of Fluid Mechanics, 39(1):419-446, 2007.

[50] J. Eggers and E. Villermaux. Physics of liquid jets. Reports on Progress in Physics, 71(3):036601, 2008.

[51] H. Lhuissier and E. Villermaux. Bursting bubble aerosols. Journal of Fluid Mechanics, 696:1-40, 2012.

[52] H. Lhuissier and E. Villermaux. 'Effervescent' atomization in two dimensions. Journal of Fluid Mechanics, 714:361-392, 2013. 
[53] P. Marmottant and E. Villermaux. On spray formation. Journal of Fluid Mechanics, 498:73-111, 2004.

[54] N. Bremond, C. Clanet, and E. Villermaux. Atomization of undulating liquid sheets. Journal of Fluid Mechanics, 585:421-456, 2007.

[55] E. Villermaux and C. Clanet. Life of a flapping liquid sheet. Journal of Fluid Mechanics, 462:341-363, 2002.

[56] N. Bremond and E. Villermaux. Atomization by jet impact. Journal of Fluid Mechanics, 549:273-306, 2006.

[57] A. Ashkin and J. M. Dziedzic. Radiation pressure on a free liquid surface. Physical Review Letters, 30(4):139-142, 1973.

[58] K. Sakai, D. Mizuno, and K. Takagi. Measurement of liquid surface properties by laser-induced surface deformation spectroscopy. Physical Review E, 63(4): 046302, 2001.

[59] A. Casner and J.-P. Delville. Giant Deformations of a Liquid-Liquid Interface Induced by the Optical Radiation Pressure. Physical Review Letters, 87(5):054503, 2001.

[60] S. A. Viznyuk and A. T. Sukhodol'skiir. Thermocapillary self-interaction of laser radiation in thin layers of an absorbing liquid. Soviet Journal of Quantum Electronics, 18(4):489-491, 1988.

[61] H. Chraïbi and J.-P. Delville. Thermocapillary flows and interface deformations produced by localized laser heating in confined environment. Physics of Fluids, 24(3):032102, 2012.

[62] C. Marangoni. Über die Ausbreitung der Tropfen einer Flüssigkeit auf der Oberfläche einer anderen. Annalen der Physik, 219(7):337-354, 1871.

[63] G. Lajoinie, E. Gelderblom, C. Chlon, M. Böhmer, W. Steenbergen, N. de Jong, S. Manohar, and M. Versluis. Ultrafast vapourization dynamics of laseractivated polymeric microcapsules. Nature Communications, 5:3671, 2014.

[64] H. Weichel. Laser Beam Propagation in the Atmosphere. SPIE Press, 1990.

[65] E. Hecht. Optics. Addison-Wesley, 2002.

[66] M. Versluis. High-speed imaging in fluids. Experiments in Fluids, 54(2):1458, 2013.

[67] A. van der Bos, A. Zijlstra, E. Gelderblom, and M. Versluis. iLIF: Illumination by Laser-Induced Fluorescence for single flash imaging on a nanoseconds timescale. Experiments in Fluids, 51(5):1283-1289, 2011.

[68] P.-G. de Gennes, F. Brochard-Wyart, and D. Quéré. Capillarity and Wetting Phenomena: Drops, Bubbles, Pearls, Waves. Springer, New York, 2002. 
[69] R. M. Pope and E. S. Fry. Absorption spectrum (380-700 nm) of pure water. II. Integrating cavity measurements. Applied Optics, 36(33):8710-8723, 1997.

[70] C. Gayathri and A. Ramalingam. Single-beam Z-scan measurement of the thirdorder optical nonlinearities of azo dyes. Spectrochimica Acta Part A: Molecular and Biomolecular Spectroscopy, 68(3):578-582, 2007.

[71] 2-Butanone (Material Safety Data Sheet). Sigma-Aldrich, 2016.

[72] R. D. Deegan, O. Bakajin, T. F. Dupont, G. Huber, S. R. Nagel, and T. A. Witten. Capillary flow as the cause of ring stains from dried liquid drops. Nature, 389 (6653):827-829, 1997.

[73] A. L. Klein. MATLAB-Devices: Collection of classes to control lab instruments such as a pulse delay generator or a syringe pump within MATLAB. https: //github.com/alexludwigklein/MATLAB-Devices, 2017.

[74] A. L. Klein. MATLAB-BeamProfiles: Collection of files and classes to handle planar laser beam profiles according to ISO 11146 and study their interaction with a spherical drop. https://github.com/alexludwigklein/ MATLAB-BeamProfiles, 2017.

[75] A. L. Klein. MATLAB-Videos: Collection of classes, functions and scripts to analyse movies in MATLAB including memory mapping for huge files. https://github.com/alexludwigklein/MATLAB-Videos, 2017.

[76] ISO 11146-1:2005(E): Lasers and laser-related equipment - Test methods for laser beam widths, divergence angles and beam propagation ratios, Part 1: Stigmatic and simple astigmatic beams, 2005.

[77] P. K. Kennedy, D. X. Hammer, and B. A. Rockwell. Laser-induced breakdown in aqueous media. Progress in Quantum Electronics, 21(3):155-248, 1997.

[78] J.-P. Delville, M. Robert de Saint Vincent, R. D. Schroll, H. Chraibi, B. Issenmann, R. Wunenburger, D. Lasseux, W. W. Zhang, and E. Brasselet. Laser microfluidics: Fluid actuation by light. Journal of Optics A: Pure and Applied Optics, 11(3):034015, 2009.

[79] L. Xu, L. Barcos, and S. R. Nagel. Splashing of liquids: Interplay of surface roughness with surrounding gas. Physical Review E, 76(6):066311, 2007.

[80] E. Villermaux and B. Bossa. Single-drop fragmentation determines size distribution of raindrops. Nature Physics, 5(9):697-702, 2009.

[81] R. Bergmann, D. Van Der Meer, S. Gekle, A. Van Der Bos, and D. Lohse. Controlled impact of a disk on a water surface: Cavity dynamics. Journal of Fluid Mechanics, 633:381-409, 2009. 
[82] S. Gekle, I. R. Peters, J. M. Gordillo, D. van der Meer, and D. Lohse. Supersonic Air Flow due to Solid-Liquid Impact. Physical Review Letters, 104(2):024501, 2010.

[83] W. Bouwhuis, R. C. A. van der Veen, T. Tran, D. L. Keij, K. G. Winkels, I. R. Peters, D. van der Meer, C. Sun, J. H. Snoeijer, and D. Lohse. Maximal Air Bubble Entrainment at Liquid-Drop Impact. Physical Review Letters, 109(26): 264501, 2012.

[84] R. W. Coons, S. S. Harilal, D. Campos, and A. Hassanein. Analysis of atomic and ion debris features of laser-produced Sn and Li plasmas. Journal of Applied Physics, 108(6):063306, 2010.

[85] P. Mora. Theoretical model of absorption of laser light by a plasma. Physics of Fluids, 25(6):1051-1056, 1982.

[86] C. R. Phipps, T. P. Turner, R. F. Harrison, G. W. York, W. Z. Osborne, G. K. Anderson, X. F. Corlis, L. C. Haynes, H. S. Steele, K. C. Spicochi, and T. R. King. Impulse coupling to targets in vacuum by $\mathrm{KrF}, \mathrm{HF}$, and $\mathrm{CO}_{2}$ single-pulse lasers. Journal of Applied Physics, 64(3):1083-1096, 1988.

[87] M. M. Basko, V. G. Novikov, and A. S. Grushin. On the structure of quasistationary laser ablation fronts in strongly radiating plasmas. Physics of Plasmas, 22(5):053111, 2015.

[88] R. Fabbro, C. Max, and E. Fabre. Planar laser-driven ablation: Effect of inhibited electron thermal conduction. Physics of Fluids, 28(5):1463-1481, 1985.

[89] J. Pstruś. Surface tension and density of liquid In-Sn-Zn alloys. Applied Surface Science, 265:50-59, 2013.

[90] R. Fabbro, J. Fournier, P. Ballard, D. Devaux, and J. Virmont. Physical study of laser-produced plasma in confined geometry. Journal of Applied Physics, 68(2): 775-784, 1990.

[91] J. R. Freeman, S. S. Harilal, B. Verhoff, A. Hassanein, and B. Rice. Laser wavelength dependence on angular emission dynamics of Nd:YAG laser-produced Sn plasmas. Plasma Sources Science and Technology, 21(5):055003, 2012.

[92] M. Masnavi, M. Nakajima, K. Horioka, H. P. Araghy, and A. Endo. Simulation of particle velocity in a laser-produced tin plasma extreme ultraviolet source. Journal of Applied Physics, 109(12):123306, 2011.

[93] S. S. Harilal, T. Sizyuk, A. Hassanein, D. Campos, P. Hough, and V. Sizyuk. The effect of excitation wavelength on dynamics of laser-produced tin plasma. Journal of Applied Physics, 109(6):1-9, 2011.

[94] R. A. Burdt, S. Yuspeh, K. L. Sequoia, Y. Tao, M. S. Tillack, and F. Najmabadi. Experimental scaling law for mass ablation rate from a Sn plasma generated by a $1064 \mathrm{~nm}$ laser. Journal of Applied Physics, 106(3):033310, 2009. 
[95] F. Dahmani and T. Kerdja. Planar laser-driven ablation model for nonlocalized absorption. Physics of Fluids B: Plasma Physics, 3(5):1232-1240, 1991.

[96] B. N. Chichkov, C. Momma, S. Nolte, F. Von Alvensleben, and A. Tünnermann. Femtosecond, picosecond and nanosecond laser ablation of solids. Applied Physics A, 63(2):109-115, 1996.

[97] E. G. Gamaly, A. V. Rode, B. Luther-Davies, and V. T. Tikhonchuk. Ablation of solids by femtosecond lasers: Ablation mechanism and ablation thresholds for metals and dielectrics. Physics of Plasmas, 9(3):949-957, 2002.

[98] S. V. Stankus, I. V. Savchenko, and A. S. Agazhanov. Thermal Conductivity and Thermal Diffusivity of Liquid Indium-Tin Alloys. International Journal of Thermophysics, 33(5):774-782, 2012.

[99] G. Cisneros, J. S. Helman, and C. N. J. Wagner. Dielectric function of liquid tin between 250 and $1100^{\circ}$ C. Physical Review B, 25(6):4248-4251, 1982.

[100] A. I. Golovashkin, I. S. Levchenko, G. P. Motulevich, and A. A. Shubin. Optical properties of indium. Soviet Physics JETP, 24(6):1093-1100, 1967.

[101] E. G. Gamaly. The physics of ultra-short laser interaction with solids at nonrelativistic intensities. Physics Reports, 508(4):91-243, 2011.

[102] J. M. Kolinski, S. M. Rubinstein, S. Mandre, M. P. Brenner, D. A. Weitz, and L. Mahadevan. Skating on a Film of Air: Drops Impacting on a Surface. Physical Review Letters, 108(7):074503, 2012.

[103] C. Josserand and S. T. Thoroddsen. Drop impact on a solid surface. Annual Review of Fluid Mechanics, 48:365-91, 2016.

[104] G. Riboux and J. M. Gordillo. The diameters and velocities of the droplets ejected after splashing. Journal of Fluid Mechanics, 772:630-648, 2015.

[105] G. K. Batchelor. An Introduction to Fluid Dynamics. Cambridge University Press, 1967.

[106] M. J. Cooker and D. H. Peregrine. Pressure-impulse theory for liquid impact problems. Journal of Fluid Mechanics, 297:193-214, 1995.

[107] A. Antkowiak, N. Bremond, S. L. Dizès, and E. Villermaux. Short-term dynamics of a density interface following impact. Journal of Fluid Mechanics, 577: 241-250, 2007.

[108] H. N. Oguz and A. Prosperetti. Bubble entrainment by the impact of drops on liquid surfaces. Journal of Fluid Mechanics, 219:143-179, 1990.

[109] H. N. Oguz and A. Prosperetti. Dynamics of bubble-growth and detachment from a needle. Journal of Fluid Mechanics, 257:111-145, 1993. 
[110] H. Power and L. C. Wrobel. Boundary Integral Methods in Fluid Mechanics. WIT Press, 1995.

[111] P. D. Hicks and R. Purvis. Air cushioning and bubble entrapment in threedimensional droplet impacts. Journal of Fluid Mechanics, 649:135-163, 2010.

[112] I. R. Peters, D. van der Meer, and J. M. Gordillo. Splash wave and crown breakup after disc impact on a liquid surface. Journal of Fluid Mechanics, 724: 553-580, 2013.

[113] A. Prosperetti. Advanced Mathematics for Applications. Cambridge University Press, 2011.

[114] D. Richard and D. Quéré. Bouncing water drops. Europhys. Lett., 50(6):769-775, 2000.

[115] A. Rozhkov, B. Prunet-Foch, and M. Vignes-Adler. Dynamics of a liquid lamella resulting from the impact of a water drop on a small target. Proceedings of the Royal Society A: Mathematical, Physical and Engineering Sciences, 460(2049): 2681-2704, 2004.

[116] C. Vernay, L. Ramos, and C. Ligoure. Free radially expanding liquid sheet in air: Time- and space-resolved measurement of the thickness field. Journal of Fluid Mechanics, 764:428-444, 2015.

[117] N. Bremond and E. Villermaux. Bursting thin liquid films. Journal of Fluid Mechanics, 524:121-130, 2005.

[118] W. E. Byerly. An Elementary Treatise on Fourier's Series and Spherical, Cylindrical and Ellipsoidal Harmonics with Applications to Problems in Mathematical Physics. Gin \& Company, 1893.

[119] I. R. Peters, Y. Tagawa, N. Oudalov, C. Sun, A. Prosperetti, D. Lohse, and D. van der Meer. Highly focused supersonic microjets: Numerical simulations. Journal of Fluid Mechanics, 719:587-605, 2013.

[120] Y. Utsunomiya, T. Kajiwara, T. Nishiyama, K. Nagayama, S. Kubota, and M. Nakahara. Laser ablation of liquid surface in air induced by laser irradiation through liquid medium. Applied Physics A, 101(1):137-141, 2010.

[121] S. R. G. Avila and C.-D. Ohl. Cavitation-induced fragmentation of an acoustically-levitated droplet. Journal of Physics: Conference Series, 656(1):012017, 2015.

[122] S. A. Reijers, J. H. Snoeijer, and H. Gelderblom. Droplet deformation by short laser-induced pressure pulses. arXiv:1702.01538 [physics.flu-dyn], 2017.

[123] M. W. Sigrist and F. K. Kneubühl. Laser-generated stress waves in liquids. The Journal of the Acoustical Society of America, 64(6):1652-1663, 1978. 
[124] X. Wang and X. Xu. Thermoelastic wave induced by pulsed laser heating: Applied Physics A Materials Science \& Processing, 73(1):107-114, 2001.

[125] A. H. Clauer, J. H. Holbrook, and B. P. Fairand. Effects of laser induced shock waves on metals. In Shock Waves and High-Strain-Rate Phenomena in Metals, pages 675-702. Springer, 1981.

[126] A. M. Marpaung, H. Kurniawan, M. O. Tjia, and K. Kagawa. Comprehensive study on the pressure dependence of shock wave plasma generation under TEA $\mathrm{CO}_{2}$ laser bombardment on metal sample. Journal of Physics D: Applied Physics, 34(5):758-771, 2001.

[127] F. E. C. Culick. Comments on a Ruptured Soap Film. Journal of Applied Physics, 31(6):1128-1129, 1960.

[128] A. Oron, S. H. Davis, and S. G. Bankoff. Long-scale evolution of thin liquid films. Reviews of modern physics, 69(3):931-980, 1997.

[129] J. B. Keller and I. Kolodner. Instability of Liquid Surfaces and the Formation of Drops. Journal of Applied Physics, 25(7):918-921, 1954.

[130] A. Braslau. Surface Roughness of Water Measured by X-Ray Reflectivity. Physical Review Letters, 54(2):114-117, 1985.

[131] H.-R. Kuhn, J. Koch, R. Hergenröder, K. Niemax, M. Kalberer, and D. Günther. Evaluation of different techniques for particle size distribution measurements on laser-generated aerosols. Journal of Analytical Atomic Spectrometry, 20(9): 894-900, 2005.

[132] S. R. Gonzalez Avila and C.-D. Ohl. Fragmentation of acoustically levitating droplets by laser-induced cavitation bubbles. Journal of Fluid Mechanics, 805: 551-576, 2016. 


\section{Samenvatting}

In deze thesis kijken we naar hoe een vloeibare druppel reageert op de inslag van een laserpuls. De laserpuls induceert een snelle en hevige faseverandering zoals we laten zien in $\S 2$ : de druppel wordt versneld, vervormt sterk en breekt uiteindelijk op. Dit proces gaat meestal samen met schokgolven in de lucht, massa-ablatie en zelfs plasma generatie. Het doel van deze thesis is het beschrijven en begrijpen van de levenscyclus van de druppel: van de initiële laser inslag, naar de vervorming van de druppel en uiteindelijk naar het uiteenspatten van de druppel in kleinere druppeltjes.

Een belangrijk aspect van deze thesis was het ontwerpen, plannen en bouwen van een experimentele opstelling, zoals gepresenteerd in $\S 3$, om de druppel nauwkeurig genoeg te kunnen sturen en volgen om de faseverandering en de dynamica goed te kunnen bestuderen. We maken hiervoor gebruik van een lasersysteem dat hoge-energetische pulsen van groen licht uitzendt gedurende enkele nanoseconden. De experimentele opstelling stelt ons in staat om verschillende druppels met een straal $R_{0}$ van enkele millimeters te genereren met verschillende kleurstofoplossingen, met elk hun eigen absorptie eigenschappen voor groen licht. De reactie van de druppel op de laserpuls wordt met twee orthogonale camera standpunten bekeken: het combineren van stroboscopische en hoge snelheidsopnamen met belichtingstijden van een paar nanoseconden laat alle relevante tijdschalen zien. Deze tijdschalen verschillen van ordegrootte van de nanoseconden duur van de laserpuls tot de vloeistof dynamische reactie van de druppel op deze puls op een tijdschaal van milliseconden.

In $\S 4$ wordt de overdracht van de impuls door de laser puls naar de druppel bestudeerd. We laten zien dat de laser inslag een zeer kleine laag aan het koken brengt aan de belichte kant van de druppel. De geabsorbeerde energie per massa eenheid in deze laag aan het druppeloppervlak is vergelijkbaar met de specifieke latente warmte van verdamping. Deze laag kookt en er wordt een schokgolf uitgezonden in de lucht, gevolgd door de emissie van damp en mist. Alhoewel de hoeveelheid verdampte massa maar een klein deel is van de totale druppel massa, zorgt de terugslagdruk toch voor een aanzienlijke versnelling. We laten zien dat de snelheid van het massamiddelpunt $U$ pro- 
portioneel is met de laser energie zodra de totale laser energie groter is dan de energie die nodig is om de laag aan het koken te brengen.

In samenwerking met het Advanced Research Center for Nanolithography (ARCNL) laten we zien dat laser inslag ook bij metaal druppels zorgt voor een druppelversnelling, zie §5. De druppel wordt echter in dit geval versneld door een expanderende plasmawolk dichtbij het oppervlak van de druppel in plaats van een mist wolk. Hoewel het voortstuwmechanisme compleet anders is vinden we ook hier een minimale energie drempel en eenzelfde schaling voor de snelheid van het massamiddelpunt $U$.

Er bestaat een duidelijke scheiding in tijdschalen die karakteristiek is voor de twee bovengenoemde voortstuwmechanismes. Initiël vindt de druppel versnelling plaats op een nanoseconde tijdschaal $\tau_{e}$, bepaald door de hoeveelheid verloren, verdampte of geïoniseerde massa. De daaropvolgende voortstuwing zorgt voor een laterale expansie van de druppel en vindt plaats op de traagheidstijdschaal $\tau_{i}=R_{0} / U$. Uiteindelijk wordt de vervorming van de druppel geremd door oppervlaktespanning, $\tau_{c}=\left(\rho R_{0}^{3} / \gamma\right)^{1 / 2}$ die typisch plaats vindt op de capillaire tijdschaal. Door de duidelijke scheiding van tijdschalen, $\tau_{e} \ll \tau_{i}<\tau_{c}$, kunnen we de laser inlag op een algemene manier behandelen: de interactie van een laserpuls met een vloeibare druppel kan succesvol worden gemodelleerd door een terugslagdrukpuls op het druppeloppervlak, zoals we in $\S 6$ laten zien. De resulterende druppelvervorming kunnen we vervolgens onderscheiden in drie stadia. Een vroegtijdig stadium waarin de druppel wordt versneld door de terugslagdruk op het oppervlak. Daarna een tussenstadium waarin de druppel vervormt in een dunne, vloeibare schijf. Uiteindelijk een laatste fase waarin de expansie wordt gelimiteerd door oppervlaktespanning. In $\S 6$ introduceren we een analytisch model voor de vroegtijdige druppel vervorming als functie van de laserpuls vorm en laser energie. Dit model stelt ons in staat om de ratio tussen de kinetische vervormingsenergie en de kinetische translatie energie uit te rekenen. We laten hierbij zien dat de maximale druppel expansie kan worden gekregen als de laserpuls compleet gefocust is in een kleine spot. Echter, de uiteindelijke druppel zal in dit geval niet symmetrisch vervormen in een dunne, vloeibare schijf, wat alleen kan met een gelijkmatige laserpuls. De vervormingsevolutie rekenen we uit met boundary-integral simulaties. We vergelijken deze simulaties met de experimentele resultaten en laten zien dat het model de druppel vervorming uitstekend voorspelt.

Met een goede beschrijving van de druppel vervorming op zak kunnen we nu focussen op de druppel fragmentatie zoals beschreven in §7. Het fragmentatie proces wordt geleid door een Rayleigh-Taylor instabiliteit, waarbij de perturbaties veroorzaakt worden door twee verschillende versnellingen, 
beiden in een andere richting en versterkt door de Rayleigh-Taylor instabiliteiten op de vervormende druppel. De eerste perturbatie wordt veroorzaakt door de versnelling van het oppervlak, waardoor destabilisatie van de schijf op late tijdschalen zichtbaar wordt. De tweede perturbatie wordt veroorzaakt door de capillaire versnelling en zorgt voor destabilisatie aan de randen van de schijf. We volgen de amplitude van beide perturbaties van de druppelvervorming op vroege en late tijden. We leggen een schalingsmodel uit voor de karakteristieke tijden en golflengtes van het opbreken. We laten zien dat er goede overeenkomst is tussen dit schalingsmodel en de experimentele data. Het opbreken van de dunne vloeistof schijf wordt uiteindelijk veroorzaakt als amplitudes van de globale Rayleigh-Taylor instabiliteit groter worden dan de lokale schijfdikte. Bovendien laten we zien dat er voorkeursgebieden zijn waarbij de vlakke schijf eerst zal doorbreken, doordat er extra radiële en azimutale afhankelijkheden zitten in de schijf, hoewel het eigenlijke opbreken een random proces is.

Samenvattend hebben we de levenscyclus van een druppel beschreven, wanneer een impulsieve faseverandering in de druppel de vloeistof dynamische reactie van de druppel bepaald. We hebben bepaald dat de lokale verdamping van het druppeloppervlak en een plasmawolk gevormd in de nabijheid van de druppel de drijvende krachten zijn voor de dynamische reactie van de druppel. De reactie van de druppel wordt beschreven als een karakteristieke verandering in de topologie: de sferische druppel vervormt tot een vloeibare schijf, welke radiël uitspreidt door de invloed van oppervlaktespanning. We geven een analytische beschrijving voor de kinematica van de druppel, welke we extensief hebben gevalideerd. Daarnaast hebben we laten zien dat de interactie van een laserpuls met een druppel goed gemodelleerd kan worden door een terugtrekdrukpuls op het vloeistofoppervlak toe te passen. De details van de laser-druppel interactie kunnen dan beschreven worden met dit drukprofiel. De fragmentatie van de druppel wordt veroorzaakt door Rayleigh-Taylor instabiliteiten, welke de groter wordende druppel destabiliseren. Hiervoor geven we een schalingsargument waarbij we gebruik maken van de karakteristieke tijd en het golfgetal van het opbreken. 
178 
—Et kütt wie et kütt en et hätt noch immer jot jejange!

-Whatever will be, will be and it

has always worked out!

Idiom from Cologne

\section{Acknowledgements}

Finishing a PhD project and writing the thesis was a challenge. The environment, both the work and private life, was of great importance to me and allowed for a success in the first place: the discussions with colleagues, advice and encouragements from friends and family, or just having a good time with a friend on the bike. I would like to express my gratitude to all people who created the environment that I was lucky to have during the last four years.

First, I would like to thank Detlef Lohse to grant me access to the academic world and the Physics of Fluids group (PoF) in particular. Detlef, we met each other while I was pursuing the idea to write a PhD thesis in industry - an idea that finally died for me in 2013. Thank you very much to have trust in me at that time, which for sure was not easy for me, and discuss opportunities for a fresh start! Throughout the last four years, you have been there to give guidance without limiting my freedom of choice. I am sure this spirit nurtures the atmosphere at PoF with its huge diversity in people, thoughts, and ideas. Dear Detlef, thank you very much!

The Physics of Fluids group at the University of Twente is a special place to me: a huge and diverse crowd of talented people who are willing to share their knowledge. I am grateful to be a part of PoF and would like to thank all its members for their support, advice, insights in the world of science, and many discussions and feedback! A few people I would like to thank in particular: Jacco Snoeijer, Michel Versluis, and Chao Sun, thank you for all the support. Claas Willem Visser, Guillaume Lajoinie, and Stefan Karpitschka, thank you for your advice and patience with me when borrowing experimental equipment. In the same line, Michiel van Limbeek, thank you for having me in your lab space at PoF. Wilco Bouwhuis, many thanks for your input and numerical simulations. A great part of the research at $\mathrm{PoF}$ is unthinkable in my opinion without the support of Joanita Leferink, Bas Benschop, GertWim Bruggert, and Martin Bos: thank you for your help and especially the understanding that you have for the short-notice requests of scientists to get their most important experiments, computers, or whatever running!

A small team of PoF people, including myself, have been located at the campus of our industrial partner ASML. My daily environment was then set by 
our team leader Hanneke Gelderblom and my PhD colleagues Marise Gielen and Sten Reijers. Hanneke, thank you very much for in-depth discussions, critical questions that always led to an improvement of my work and your ability to rip apart my writing and put it together such that it makes sense. You taught me a lot and I am grateful for the time you invested in my PhD thesis! Marise and Sten, I enjoy discussing and working with you. You have a part in my thesis for your daily support and feedback, and hectoliters of tea produced and, more importantly, shared with me! Sten, thank you for our trip with Jonas to New York after APS-DFD 2015. Also, I would like to thank Riëlle de Ruiter, who was part of the team during her postdoc, for tips and tricks on chemicals. Koen Arens, I enjoyed to have you at my setup during your master thesis. All of you, many thanks and I am looking forward to one of our next group dinners!

The industrial partner not only funded a great part of my research but also supported my thesis by its many people at the ASML campus. Ramin Badie, Ben de Smet, Wim van der Zande, and Joep Pijnenburg supported me as group leaders. Michel Riepen, Maarten van Kampen, Andrei Yakunin, Luigi Scaccabarozzi, Ronald Boot and many others gave helpful input that lead to fruitful discussions. I had the pleasure to be in a lab next to two former PoF colleagues: Koen Winkels and Theo Driessen. Many thanks for discussions, often on short notice during coffee breaks. Special thanks to Theo for introducing me to race biking with Lukas and Balder! During my first weeks at ASML, I followed Adam Lassise around, a great experimentalist, who introduced me to the labs at ASML. The campus of ASML also got me in touch with many other scientists such as Chris Lee, who I would like to express my gratitude for his input and ideas on my experimental setup.

The close collaboration with Emmanuel Villermaux and Henri Lhuissier lasted throughout the complete thesis, was very productive, and at the same time very enjoyable. Dear Emmanuel and Henri, thank you for your tremendous contribution to my thesis! Furthermore, thank you for having me in Marseilles for an inspiring week of discussions and a wonderful dinner! I hope our path cross again in future collaborations.

The collaboration with the Advanced Research Center for Nanolithography (ARCNL) in Amsterdam was very fruitful for my thesis. The combination of our experimental setups to study the physics of the laser impact on a drop allowed us to explore a parameter domain that could not be covered by each individual setup. Dmitry Kurilovich, Francesco Torretti, Ronnie Hoekstra, Wim Ubachs, and Oscar Versolato: thank you very much for this collaboration! I enjoyed our meetings and discussions, especially the combination of fluid dynamics and plasma physics. 
Over the past years, I have been fortunate to have the support of my family and friends. Dear Christina, thank you for being such a great sister, you always left me in a good mood! My parents, Inge and Albert, many thanks for supporting my life choices on the road that led me to this thesis. I would also like to thank my family members and friends for their kölsche spirit: Et kütt wie et kütt en et hätt noch immer jot jejange! Along my road, I made great friends that inspired me, gave me advice, and put my view on science and life into another perspective. Meeting all of you made my life so much richer! Dear friends, thank you for being in my life.

A person that I want to thank for much more than just the last four years is Silvia Baumeier, my girlfriend at the start of the PhD and since $20^{\text {th }}$ September 2014 my wife. Dear Silvia, I could not have done this work without you. We know each other since 2002 and I am grateful for each single day that we have spent together. Thank you for all your love, encouragement, advice, and especially your patience!

Alexander L. Klein Eindhoven, May 2017 


\section{About the author}

Alexander Ludwig Klein was born in Bad Honnef, Germany, on December $1^{\text {st }}$ 1981. After graduating from high school in 2001, he completed civilian service before moving to Aachen to begin his studies of Mechanical Engineering at the RWTH Aachen University. One complementary year of his studies took place at Imperial College London, participating in the European student exchange program (ERASMUS). To complete his studies he served a six month internship in the automotive research group at Robert Bosch Company in Stuttgart. He obtained his master's degree in Mechanical Engineering in 2009 with a thesis about heat, mass, and momentum transfer at an oscillating gasfilled bubble, and was rewarded with the Springorum-Denkmünze as one of RWTH Aachen University's best graduates of the year. After his graduation he returned to Robert Bosch Company in Stuttgart where he worked for three years in the fluid dynamics and thermodynamics research group. In 2013 he started the PhD research in the Physics of Fluids group at University of Twente, supervised by Detlef Lohse and Hanneke Gelderblom. The research was part of an industrial partnership program of FOM and ASML and was performed at the ASML research facilities in Eindhoven. His submission to the Gallery of Fluid Motion was rewarded with the Milton van Dyke Award 2014 for the combination of striking visual qualities and scientific interest. His video contribution "Liquid Jellyfish \& Piercing Drops" won in the category fine work of the Slow Motion Video Award 2016. 

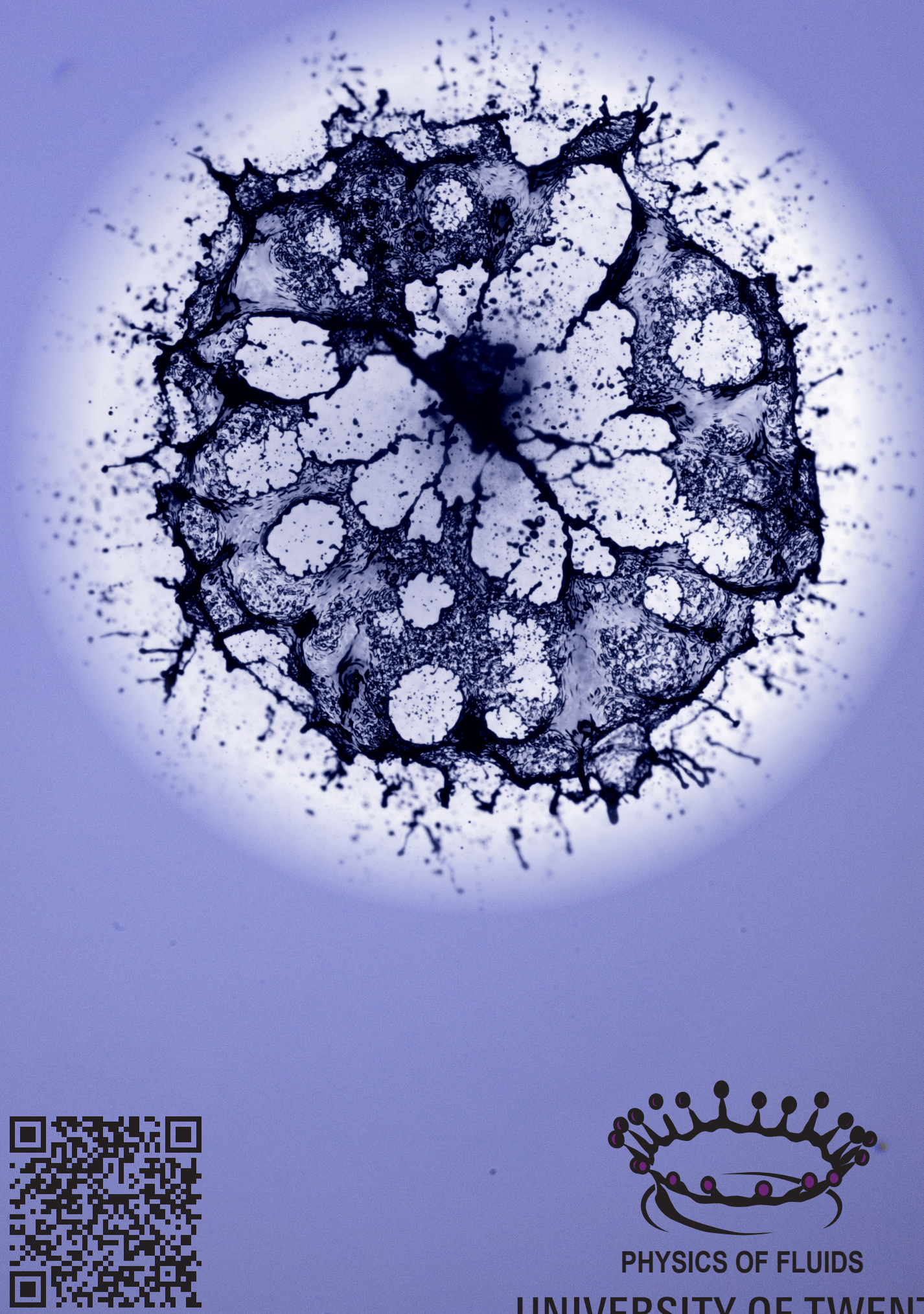

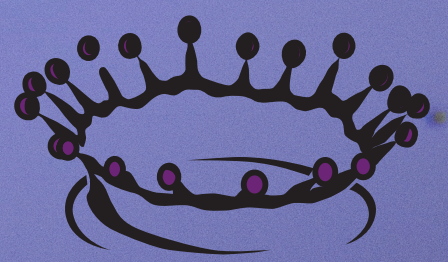

PHYSICS OF FLUIDS UNIVERSITY OF TWENTE. 